\title{
Optimized Network Dimensioning and Planning for WiMAX Technology
}

A thesis submitted in partial fulfilment

of the requirement for the degree of Doctor of Philosophy

Vitaly Teterin

January 2012

\section{Cardiff University \\ School of Computer Science \& Informatics}




\section{Declaration}

This work has not previously been accepted in substance for any degree and is not concurrently submitted in candidature for any degree.

Signed $\ldots \ldots \ldots \ldots \ldots \ldots \ldots \ldots \ldots \ldots \ldots \ldots \ldots \ldots \ldots \ldots \ldots+$ candidate $)$

Date

\section{Statement 1}

This thesis is being submitted in partial fulfillment of the requirements for the degree of $\mathrm{PhD}$.

Signed (candidate)

Date

\section{Statement 2}

This thesis is the result of my own independent work/investigation, except where otherwise stated. Other sources are acknowledged by explicit references.

Signed (candidate)

Date

\section{Statement 3}

I hereby give consent for my thesis, if accepted, to be available for photocopying and for inter-library loan, and for the title and summary to be made available to outside organisations.

Signed (candidate)

Date 


\section{Abstract}

In order to meet demands in mobile broadband and to bridge the digital divide a new technology, namely WiMAX, was introduced in 2004. However, in order to increase the financial return on the investment in WiMAX, service operators need to make every effort in designing and deploying the most cost-effective networks.

This thesis presents a novel dimensioning technique for WiMAX technology which takes the dimensioning problem to a new level and produces more accurate results in comparison to the traditional methods. Furthermore, a novel decomposed optimization framework for the WiMAX network planning is introduced which subdivides the overall problem into three distinct stages consisting of the network dimensioning stage which besides the primary task of evaluating the financial requirements produces a good starting network solution for the subsequent stages (Stage 1), initial sectorization and configuration of the network (Stage 2) and final network configuration (Stage 3). The proposed framework also solves two fundamental problems, which are cell planning and frequency planning, simultaneously. The feasibility of the final network solutions are then evaluated by OPNET simulator. 


\section{Acknowledgements}

I would like to express my gratitude and appreciation to my supervisors, Prof. Steve Hurley and Dr. Stuart Allen for their help and encouragement. I would also like to say that I was very lucky to be supervised by Prof. Steve Hurley as he is a very special person, he has been my inspiration over the course of this work, and I would like to thank him for his great patience, enormous support in all aspects, for having faith in me and keeping me positive all the way. I would like to thank the best Mum in the world, who made this all possible and gave me wings when I needed it most. Finally many thanks to my friends Dr Ahmed Alazzawi, Dr Irina Harris and future Dr Ehab El-Gindy for their help and emotional support, and also to all our beautiful and special ladies in the office Helen Williams, Pamela Munn and Kirsty Hall.

I would like to dedicate this thesis to my Mum with all my love. 


\section{Contents}

Abstract ii

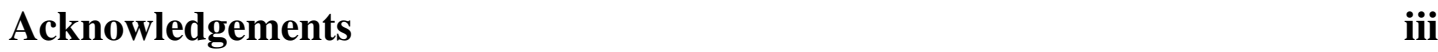

Contents iv

\begin{tabular}{ll}
\hline List of Figures & ix
\end{tabular}

List of Tables $\quad$ xiv

\begin{tabular}{|l|l}
\hline List of Algorithms & xvii
\end{tabular}

\begin{tabular}{ll}
\hline List of Acronyms & xviii
\end{tabular}

\begin{tabular}{ll}
\hline List of Notations & xxii
\end{tabular}

$\begin{array}{lll}1 & \text { Introduction } & 1\end{array}$

\begin{tabular}{|lll}
\hline 2 & Technical background and literature review & 7
\end{tabular}

2.1 WiMAX technology $\ldots \ldots \ldots \ldots \ldots$

2.2 Mobile WiMAX technical specifications . . . . . . . . . . . 9 
2.3 Network planning and optimization issues . . . . . . . . . . . . . 14

$2.3 .1 \quad$ Network survey $\ldots \ldots \ldots \ldots$. . . . . . . . . . . . . 14

2.3 .2 Network dimensioning . . . . . . . . . . . . 16

2.3 .3 RF optimization $\ldots \ldots \ldots \ldots \ldots$

\begin{tabular}{|lll}
3 & Optimization \& network model & 27
\end{tabular}

$3.1 \quad$ System Model $\ldots \ldots \ldots \ldots$. . . . . . . . . . . . . . . . 28

$3.1 .1 \quad$ Calculating horizontal and vertical loss $\ldots \ldots . . . . . .32$

3.2 Propagation models for WiMAX at $3.5 \mathrm{GHz} \ldots \ldots . \ldots 38$

$3.2 .1 \quad$ Types of propagation models . . . . . . . . . . . . . 39

3.2.2 Rapid Pipeline Development Tool . . . . . . . . . . . . 41

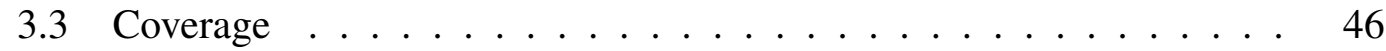

3.4 Service and Traffic $\ldots \ldots \ldots$. . . . . . . . . . . . . 47

3.5 Transmit power and subchannelization . . . . . . . . . . . . . 49

3.5.1 WiMAX transmission power . . . . . . . . . . 49

3.5 .2 Subchannelization ................. 50

3.5 .3 DL PUSC structure . . . . . . . . . . . . . 53

3.5 .4 Slot assignment formulation . . . . . . . . . . . 58

3.5 .5 Power allocation $\ldots \ldots \ldots \ldots 61$

3.6 Interference calculation . . . . . . . . . . . . . . 61

3.7 Throughput estimation $\ldots \ldots \ldots \ldots$. . . . . . . . . . . . . . 64

3.8 Transmission overheads and cell capacity $\ldots \ldots$. . . . . . . . 66 
\begin{tabular}{|ccc}
4 & Optimization - General Framework & 69
\end{tabular}

4.1 Optimization framework background . . . . . . . . . . . . . . 69

$4.1 .1 \quad$ Input data $\ldots \ldots \ldots \ldots \ldots \ldots \ldots$

4.1 .2 Initial network . . . . . . . . . . . . . 74

4.1 .3 Simulated Annealing configurations . . . . . . . . . 75

4.1 .4 Move generation strategy $\ldots \ldots \ldots$. . . . . . . . . 80

4.1 .5 Network objectives and cost function . . . . . . . . . 84

4.1 .6 Key performance indicators . . . . . . . . . . . . 88

$4.1 .7 \quad$ Neighbourhood moves: generating new solutions . . . . . . 90

4.2 AFP formulation $\ldots \ldots \ldots . \ldots . \ldots . \ldots 9$

4.3 Problem Decomposition $\ldots \ldots$. . . . . . . . . . . . . . . . 94

$4.3 .1 \quad$ Background $\ldots \ldots \ldots \ldots$. . . . . . . . . . . . 96

4.3 .2 Decomposition approach . . . . . . . . . . . . 97

5 Optimization and Dimensioning - Stage 1 100

5.1 Interference and throughput calculation $\ldots \ldots . \ldots . \ldots 103$

5.2 Capacity estimation for omni-directional base stations . . . . . . . . . 108

5.3 Operational moves . . . . . . . . . . . . . . . . . . . 109

5.4 Stage objectives and cost function weights $\ldots \ldots \ldots$. . . . . . . 121

5.5 Output of Stage 1 . . . . . . . . . . . . . . . . . 122 
6 Optimization - Stage 2 \& Stage 3 125

$6.1 \quad$ Stage 2 . Sectorization . . . . . . . . . . . . . . . . 125

6.1 .1 Optimization algorithm . . . . . . . . . . . . 126

6.1 .2 Initial sectorization of cells . . . . . . . . . . . . 128

6.1 .3 Operational moves . . . . . . . . . . . . . . 137

6.1 .4 Base station PermBase allocation procedure . . . . . . . . . 138

6.1.5 Slot assignment and interference calculation . . . . . . . . . 139

$6.1 .6 \quad$ Stage objectives and cost function weights . . . . . . . . . . 139

6.1 .7 Output of Stage $2 \ldots \ldots \ldots \ldots$

6.2 Stage 3. Final optimization . . . . . . . . . . . . . . . . . . 147

$6.2 .1 \quad$ Stage objectives and cost function weights . . . . . . . . . . 147

$6.2 .2 \quad$ Example output of Stage 3 . . . . . . . . . . . . . 149

7 Results \& Discussion 155

$7.1 \quad$ Experimental Network Scenario $\ldots \ldots$. . . . . . . . . . . 156

7.2 Framework results $\ldots \ldots \ldots \ldots 16 \ldots$

7.3 Network planning without consideration of PermBase assignment . . 181

7.4 Network planning with simplified Stage 2 operation . . . . . . . . . . 184

7.5 Network planning with different interference settings in Stage 1 . . 188

$7.6 \quad$ Network planning employing standard approach . . . . . . . . . . . . 195

7.7 Analysis of the results . . . . . . . . . . . . . . . . . . 209 
\begin{tabular}{lll}
\hline Simulation & 216
\end{tabular}

$8.1 \quad$ OPNET Wimax model $\ldots \ldots \ldots \ldots$. . . . . . . . . . . 217

8.2 Simulation approach $\ldots \ldots \ldots \ldots \ldots$

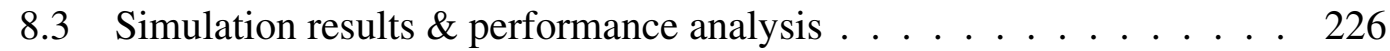

$\begin{array}{lll}9 & \text { Conclusions and future work } & \mathbf{2 3 0}\end{array}$

9.1 Future work . . . . . . . . . . . . . . . . 233

\begin{tabular}{ll}
\hline Bibliography & 236
\end{tabular} 


\section{List of Figures}

$3.1 \quad$ Horizontal and vertical planes of 60 degrees directional antenna pattern 33

3.2 Horizontal and vertical planes of omni-directional antenna pattern . . 33

3.3 Vertical angle calculation - scheme 1 . . . . . . . . . 35

3.4 Vertical angle calculation - scheme $2 \ldots \ldots 35$

3.5 RDP application . . . . . . . . . . . . . . . . 43

$3.6 \quad 3 \mathrm{D}$ visualisation of an example network terrain $\ldots . . . . . . .444$

$3.7 \quad$ Path losses base station and test points calculated using ITU-R P.452 module in RPD . . . . . . . . . . . . . . . . . . . . . . 45

3.8 OFDMA $\ldots \ldots \ldots \ldots \ldots \ldots \ldots$

3.9 DL PUSC permutation sequence [from [104]] . . . . . . . . . . . . . 54

3.10 DL PUSC physical clustering [from [104]] . . . . . . . . . . . . . 54

3.11 DL PUSC grouping clusters [from [104]] . . . . . . . . . . . . . . . 56

3.12 DL PUSC distribution of subcarriers [from [104]] . . . . . . . . . . . 57

3.13 DL PUSC - positioning of pilot subcarriers [from [104]] . . . . . . . 57

3.14 Subcarriers of subchannel plotted against group subcarrier index [from

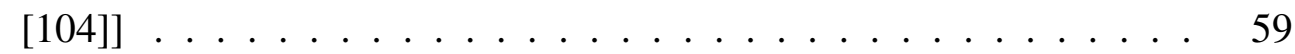


3.15 Subcarriers of subchannel plotted against absolute subcarrier index [from $[104]] \ldots \ldots$. . . . . . . . . . . . . . . . . . . . . . . 60

3.16 Downlink bandwidth calculation . . . . . . . . . . . . . 67

4.1 Optimization framework $\ldots \ldots \ldots \ldots$. . . . . . . . . . 74

4.2 Decomposition of an overall problem into sub-problems . . . . . . . 97

5.1 Initial coverage stage scheme . . . . . . . . . . . . . . 102

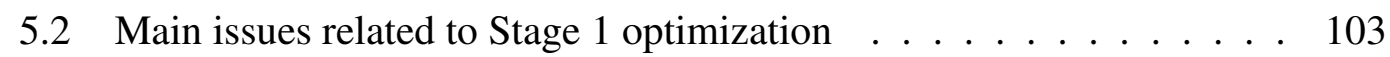

5.3 Sectorization of omni-directional base stations - evaluation of the interfering sectors. Square with red mark represents the serving base station, with black square in the centre - interfering base station, and green circle - a user $\ldots \ldots \ldots$. . . . . . . . . . . . 105

5.4 Identification of interfering sectors at omni-directional base stations . 106

5.5 Original network before deactivating base station . . . . . . . . . . . 114

5.6 Network after deactivating base station . . . . . . . . . . . . . . . . . 114

5.7 Original omni-directional pattern - angled view $\ldots \ldots$. . . . . . . 117

5.8 Original omni-directional pattern - side view . . . . . . . . . . . . . . 117

5.9 Tilted omni-directional pattern (14 degrees) - angled view . . . . . . . 118

5.10 Tilted omni-directional pattern (14 degrees) - side view . . . . . . . . 118

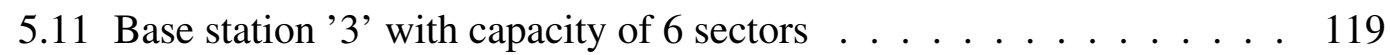

5.12 Base station '3' with capacity of 2 sectors . . . . . . . . . . . . . 119

5.13 Normalized acceptance ratios of moves for dynamic and fixed strategies 121

5.14 Stage 1 network output display $\ldots \ldots$. . . . . . . . . . . 123 
$6.1 \quad$ Stage 2 procedure . . . . . . . . . . . . . . . . . 127

6.2 Omni cell structure before sectorization . . . . . . . . . . . . . . 130

6.3 Identifying the centre of mass for the omni cell $\ldots$. . . . . . . . 130

6.4 Partitioning the omni cell on two zones . . . . . . . . . . . . . . . 130

6.5 Azimuth limitations of zone 1 in sectorization process . . . . . . . . . 132

6.6 Azimuth limitations of zone 2 in sectorization process . . . . . . . . . 132

6.7 Initial sectorization of omni base station with default configurations . 135

6.8 The resulting sectorized cell after a series of modifications . . . . . . 135

6.9 Comparison of omni cell structure to sectorized solution $\ldots . . . . \quad 135$

6.10 Network instance from the optimization Stage 1 . . . . . . . . . . . . 141

6.11 Network instance from the optimization Stage 2 . . . . . . . . . . . . 141

6.12 Cell-by-cell sectorization score results in Stage 2 . . . . . . . . . 145

6.13 Overall score results in Stage 2 . . . . . . . . . . . . . . . 146

6.14 Stage 3 procedure . . . . . . . . . . . . . . . . . . 148

6.15 Network instance from the optimization Stage 2 . . . . . . . . . . 150

6.16 Comparison of resulting network plans from Stage 2 and 3 . . . . . . 150

$7.1 \quad$ Newcastle city . . . . . . . . . . . . . . . . . . 157

7.2 Stage 1 network plan $-\mathrm{s} 1-\max \ldots \ldots \ldots 6$

7.3 Stage 1 network plan $-\mathrm{s} 1-\operatorname{dev}(+) \ldots \ldots . \ldots . \ldots 166$

7.4 Stage 1 network plan - s1-mean . . . . . . . . . . . . . . 167

7.5 Stage 1 network plan $-\mathrm{s} 1-\operatorname{dev}(-) \ldots \ldots$. . . . . . . . . 167 
7.6 Stage 1 network plan $-\mathrm{s} 1-\mathrm{min}$

$7.7 \quad$ Stage 2 network plan $-\mathrm{s} 2-1 \ldots \ldots \ldots$. . . . . . . . . 172

7.8 Stage 2 network plan $-\mathrm{s} 2-2 \ldots \ldots \ldots \ldots$. . . . . . . . . . . 172

7.9 Stage 2 network plan $-\mathrm{s} 2-3 \ldots \ldots \ldots$. . . . . . . . . . 173

7.10 Stage 2 network plan $-\mathrm{s} 2-4 \ldots \ldots$. . . . . . . . . . . 173

7.11 Stage 2 network plan $-\mathrm{s} 2-5$. . . . . . . . . . . . . . . . 174

7.12 Stage 3 network plan $-\mathrm{s} 3-1-\mathrm{min} \ldots \ldots . \ldots . \ldots 178$

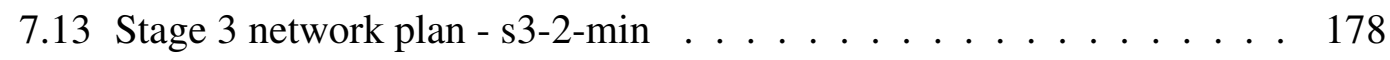

7.14 Stage 3 network plan - s3-3-min . . . . . . . . . . . . . 179

7.15 Stage 3 network plan $-\mathrm{s} 3-4-\mathrm{min}$. . . . . . . . . . . . . . 179

7.16 Stage 3 network plan $-\mathrm{s} 3-5-\mathrm{min}$. . . . . . . . . . . . . 180

7.17 Stage 1 network plan $-\mathrm{s} 1-\mathrm{i} 05$. . . . . . . . . . . . . . 192

7.18 Stage 1 network plan $-\mathrm{s} 1-\mathrm{i} 6$

7.19 Stage 2 network plan $-\mathrm{s} 2-\mathrm{i} 05$. . . . . . . . . . . . . 193

7.20 Stage 2 network plan $-\mathrm{s} 2-\mathrm{i} 6 \quad \ldots \ldots$. . . . . . . . . . 193

7.21 Stage 1 standard approach network plan - s1-3s-max $\ldots$. . . . . . . . 199

7.22 Stage 1 standard approach network plan - s1-3s-dev(+) . . . . . . . . 199

7.23 Stage 1 standard approach network plan - s1-3s-mean . . . . . . . . . 200

7.24 Stage 1 standard approach network plan - s1-3s-dev(-) . . . . . . . . 200

7.25 Stage 1 standard approach network plan -s1-3s-min . . . . . . . . . . 201

7.26 Stage 3 standard approach network plan -s3-3s-1 . . . . . . . . . 206

7.27 Stage 3 standard approach network plan -s3-3s-2 . . . . . . . . . 207 
7.28 Stage 3 standard approach network plan $-\mathrm{s} 3-3 \mathrm{~s}-3 . \ldots 207$

7.29 Stage 3 standard approach network plan - s3-3s-4 . . . . . . . . . . . 208

7.30 Stage 3 standard approach network plan $-\mathrm{s} 3-3 \mathrm{~s}-5$. . . . . . . . . . 208

7.31 Comparison of the service levels achieved by different experiments (\%) 211

7.32 Comparison of the profit levels achieved by different experiments (€) 213

7.33 Comparison of the spectral efficiency levels achieved by different ex-

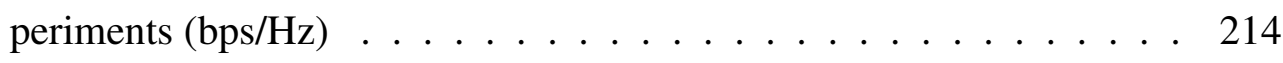

7.34 Comparison of the cost of traffic levels achieved by different experi$\operatorname{ments}(€ / \mathrm{Kbps})$

8.1 WiMAX BS node attributes . . . . . . . . . . . . . . . . 219

8.2 WiMAX SS node attributes . . . . . . . . . . . . . . 220

8.3 WiMAX Config node attributes . . . . . . . . . . . 221

8.4 OPNET antenna editor -90 degrees pattern example. . . . . . . . . . 222

8.5 WiMAX network topology example in OPNET . . . . . . . . . . . . 224

8.6 Total network throughput of the network plan 1 in OPNET simulation 228

8.7 Graphical comparison of the results between optimization framework and OPNET simulation for 10 selected network plans in terms of the service level in $\mathrm{Mbps}$. . . . . . . . . . . . . . . . . . . . . 229 


\section{List of Tables}

3.1 An example of horizontal and vertical loss table for 60 degrees directional antenna pattern $\ldots \ldots \ldots \ldots$. . . . . . . . . . 36

3.2 An example of horizontal and vertical loss calculation for 60 degrees directional antenna pattern $\ldots \ldots \ldots \ldots$

3.3 Receiver sensitivity $(\mathrm{dBm}) \ldots \ldots \ldots$. . . . . . . . . . . 47

3.4 Slot structure $\ldots \ldots \ldots \ldots \ldots$

3.5 Parameters of DL distributed subcarrier permutation (PUSC) . . . . 53

3.6 Total BS and subchannel power levels . . . . . . . . . . . . . . 61

3.7 SNR thresholds based on modulation and coding rate . . . . . . . 66

3.8 Mobile WiMAX PHY parameters . . . . . . . . . . . . . . . 67

$5.1 \quad$ Number of interfering sectors as a function of capacity of omni BS and distance between user and interfering site . . . . . . . . . . . . . 104

5.2 Default settings for omni-directional base station $\ldots \ldots$. . . . . . 111

5.3 Variation in the number of serviced users before and after deactivation of site in Stage 1 . . . . . . . . . . . . . . . . 115 
5.4 Variation in the number of serviced users before and after changing the capacity of BS from 6 to 2 sectors in Stage 1 . . . . . . . . . . . . . . 120

5.5 Network results breakdown after Stage 1 . . . . . . . . . . . . . 123

5.6 Key performance indicators of Stage 1 . . . . . . . . . . . . . . 124

6.1 Performance comparison of Stage 1 and Stage 2 for a cell . . . . . . . 136

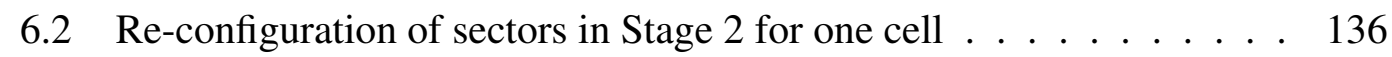

6.3 Key performance indicators comparison between Stage 1 and 2 . . . . 142

6.4 Comparison of the number of covered and serviced users between Stage 1 and $2 \ldots \ldots \ldots \ldots \ldots$. . . . . . . . . . . . . . . . . . . .

6.5 Key performance indicators comparison between Stage 1,2 and 3 . . 151

6.6 Re-configuration of sectors in Stage 3 (sites 1-4) . . . . . . . . . . . . 152

6.7 Re-configuration of sectors in Stage 3 (sites 5-9) . . . . . . . . . . 153

6.8 Comparison of the number of covered and serviced users between Stages 2 and $3 \ldots \ldots \ldots \ldots \ldots$. . . . . . . . . . . . . 154

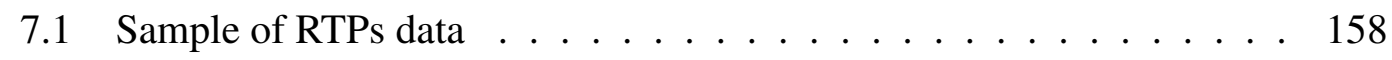

7.2 Sample of site locations data $\ldots \ldots \ldots$. . . . . . . . . . 158

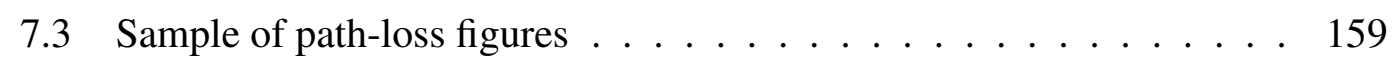

7.4 Network main parameters . . . . . . . . . . . . . . . . . . 160

7.5 Stage 1 generated networks - part 1 . . . . . . . . . . . 163

$7.6 \quad$ Stage 1 generated networks - part 2 . . . . . . . . . . . . . 164

7.7 Stage 1 selected networks for subsequent modifications . . . . . . . . 165

7.8 Stage 2 generated networks $\ldots \ldots \ldots \ldots$. . . . . . . . . 170 
7.9 Stage 2 statistical analysis of generated networks $\ldots \ldots$. . . . . 171

7.10 Stage 3 generated networks . . . . . . . . . . . . . . 176

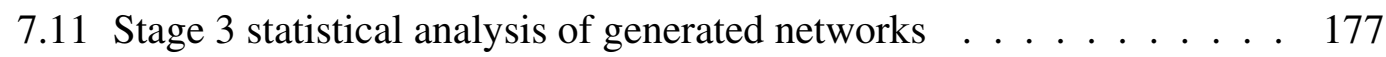

7.12 Stage 3 generated networks without PermBase assignment . . . . . . 182

\begin{tabular}{lll}
\hline 7.13 & Stage 3 statistical analysis of networks without PermBase assignment & 183
\end{tabular}

7.14 Sectorized omni networks with simplified Stage 2 operation . . . . . 185

7.15 Stage 3 generated networks after simplified Stage 2 operation . . . . . 186

7.16 Stage 3 statistical analysis of networks after simplified Stage 2 operation 187

7.17 Generated networks with different interference settings in Stage 1 . . 190

7.18 Stage 2 generated networks after Stage 1 with different interference settings . . . . . . . . . . . . . . . . . 191

7.19 Stage 2 statistical analysis of generated networks after Stage 1 with different interference settings . . . . . . . . . . . . . . . 194

7.20 Stage 1 generated networks with standard approach - part 1 . . . . . 196

7.21 Stage 1 generated networks with standard approach - part 2 . . . . . 197

7.22 Stage 1 selected networks generated with standard approach $\ldots$. . . 198

7.23 Stage 2 generated networks with standard approach . . . . . . . . . 203

7.24 Stage 3 generated networks with standard approach . . . . . . . . . . 204

7.25 Stage 3 statistical analysis of generated networks with standard approach 205

$8.1 \quad$ Network plans selected for simulation in OPNET . . . . . . . . . . . 227

8.2 Comparison of the results in terms of service between optimization framework and OPNET simulation . . . . . . . . . . . . . . . 228 


\section{List of Algorithms}

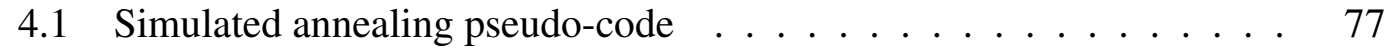

4.2 Pseudo-code for move selection scheme . . . . . . . . . . . . . . 83

4.3 Pseudo-code for move selection scheme . . . . . . . . . . . . . . . 84

$5.1 \quad$ SINR calculation pseudo-code for omni-directional base station $\ldots 108$

5.2 Capacity estimation procedure for omni-directional antennas - Part 1 . 110

5.3 Capacity estimation procedure for omni-directional antennas - Part 2. . 111

5.4 Omni-directional base station activation procedure . . . . . . . . 112

5.5 Power change pseudo-code for omni-directional base station . . . . . 116

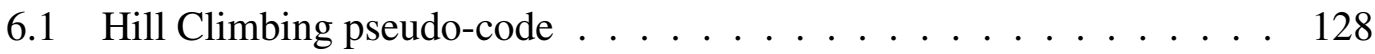

6.2 Slot assignment algorithm . . . . . . . . . . . . . . . 140 


\section{List of Acronyms}

2G Second Generation

3G Third Generation

AAS Adaptive Antenna System

ACP Automatic Cell Planning

AFP Automatic Frequency Planning

AMC Adaptive Modulation and Coding

BER Bit Error Rate

BS Base Station

CapEX Capital Expenditure

CCI Co-Channel Interference

CDMA Code Division Multiple Access

CP cyclic prefix

CSI Channel State Information

DL Downlink

DL FUSC DL Fully Used Subcarriers 
DL PUSC DL Partially Used Subcarriers

DOCSIS Data Over Cable Service Interface Specification

DSL Digital Subscriber Line

EIRP Effective Isotropic Radiated Power

EMC Electromagnetic Compatibility

EVDO Evolution-Data Optimized

FDD Frequency Division Duplex

FD-TDMA Frequency-Division Time-Division-Multiple-Access

GSM Global System for Mobile Communications

HARQ Hybrid Automatic Repeat Request

HC Hill Climbing

HSDPA High-Speed Downlink Packet Access

HSPA High-Speed Packet Access

HSUPA High-Speed Uplink Packet Access

ICI Inter-Cell Interference

IEEE Institute of Electrical and Electronics Engineers

IFFT Inverse Fast Fourier Transform

ISI Inter-Symbol Interference

KPI Key Performance Indicators

LOS Line-of-Sight 
MAC Media Access Control

MIMO Multiple in and multiple out

NLOS Non-Line-of-Sight

OFDM Orthogonal Frequency Division Multiplexing

OFDMA Orthogonal Frequency Division Multiple Access

OpEX Operating Expenditure

PermBase Permutation Base

PHY Physical layer

QAM Quadrature Amplitude Modulation

QoS Quality of Service

QPSK Quadrature Phase Shift Keying

RF Radio Frequency

RPD Rapid Pipeline Development

RTP Reception Test Point

SA Simulated Annealing

SINR Signal to Interference-plus-Noise Ratio

SNR Signal to Noise Ratio

SOFDMA Scalable OFDMA

TDD Time Division Duplex

UL Uplink 
UMTS Universal Mobile Telecommunications System

WCDMA Wideband Code Division Multiple Access

WiMAX Worldwide Interoperability for Microwave Access

Wi-Fi Wireless Fidelity 


\section{List of Notations}

\begin{tabular}{|c|c|}
\hline$\delta_{j}^{i}$ & Binary assignment status of slot $L_{i}$ to RTP $R_{j}$ \\
\hline$\delta_{j-k}^{i}$ & Binary usage status of slot $L_{i}$ at sector $B_{j-k}$ \\
\hline$\epsilon_{\mathrm{col}}$ & Average ratio of collisions in a single slot \\
\hline$\gamma_{i}^{j-k}$ & SINR received by user at RTP $R_{i}$ from serving sector $B_{j-k}$ \\
\hline$\gamma_{i}$ & SINR received by user at RTP $R_{i}$ \\
\hline$\psi$ & $\begin{array}{l}\text { Angle between the main beam of antenna and a user in the horizontal } \\
\text { plane }\end{array}$ \\
\hline$\theta$ & Angle between the main beam of antenna and a user in the vertical plane \\
\hline$\Omega$ & Solution space \\
\hline$B$ & Set of sectors \\
\hline$B_{i-j}^{A}$ & Type of sector antenna $i$ at site $S_{j}$ \\
\hline$B_{i-j}^{G}$ & Gain of sector antenna $i$ at site $S_{j}$ \\
\hline$B_{i-j}^{\alpha}$ & Tilt angle of sector antenna $i$ at site $S_{j}$ \\
\hline$B_{i-j}^{\beta}$ & Azimuth angle of sector antenna $i$ at site $S_{j}$ \\
\hline$B_{i-j}^{C A P E X}$ & Cost of sector $i$ at site $S_{j}$ \\
\hline
\end{tabular}


$B_{j-k}^{c a p} \quad$ DL capacity of base station sector $B_{j-k}$

$B^{\text {eval }} \quad$ Set of sectors for capacity re-evaluation

$B_{i-j}^{L_{h o r}} \quad$ Horizontal losses of sector antenna $i$ at site $S_{j}$

$B_{i-j}^{L_{v e r t}} \quad$ Vertical losses of sector antenna $i$ at site $S_{j}$

$B_{i-j}^{P} \quad$ Power of sector antenna $i$ at site $S_{j}$

$b_{m} \quad$ Modulation gain

$B_{i-j} \quad$ Sector $i$ at site $S_{j}$

$b_{i-j} \quad$ Binary operational status of sector $i$ at site $S_{j}$

$B_{j-k}^{\text {PermBase }} \quad$ Permutation base of sector $j$ at site $S_{k}$

$B_{S_{k}} \quad$ Set of sectors at site $S_{k}$

$C_{\text {slot }}^{D L} \quad$ Slot capacity

$C_{\text {subfr }}^{D L} \quad$ Theoretical throughput of the DL subframe

$c_{r} \quad$ Modulation coding rate

E $\quad$ Energy of a solution state

$F_{j-k}\left(R_{i}\right) \quad$ Downlink received signal strength from sector $B_{j-k}$ to RTP $R_{i}$

$I_{j-k}^{x}\left(R_{i}\right) \quad$ Interference level at RTP $R_{i}$ from the cell $B_{j-k}$ on slot $L_{x}$

$I_{j-k}^{o m n}\left(R_{i}\right) \quad$ Total interference level at RTP $R_{i}$ from the cell $B_{j-k}$ in Stage 1 (with omni antennas)

$I_{j-k}\left(R_{i}\right) \quad$ Total interference level at RTP $R_{i}$ from the cell $B_{j-k}$

$L \quad$ Set of slots

$M \quad$ Total traffic mass of the network 
$N_{\text {slots }} \quad$ Total number of slots in the DL subframe

$N_{\text {smb_slot }} \quad$ Number of symbols per slot

$N_{\text {subcar }} \quad$ Total number of subcarriers

$N_{\text {symbols }} \quad$ Number of symbols in the DL subframe

$p \quad$ Move acceptance probability

$Q_{i-j} \quad$ Propagation loss between $S_{i}$ and $R_{j}$

$R \quad$ Set of reception test points

$R_{i}^{\tau} \quad$ Traffic demand at RTP $R_{i}$

$R_{i}^{r e v} \quad$ Revenue generated by serving RTP $R_{i}$

$R_{i}^{G} \quad$ Gain of user antenna at RTP $R_{i}$

$R_{i}^{\tau}\left(L_{j}\right) \quad$ Achievable bit-rate on slot $L_{j}$ at RTP $R_{i}$

$R_{i}^{\text {cov }} \quad$ Binary coverage status of RTP $R_{i}$

$R_{i}^{R x} \quad$ Receiver sensitivity at RTP $R_{i}$

$R_{i}^{\text {serv }} \quad$ Binary service status of RTP $R_{i}$

$R_{i-j-k}^{c o v} \quad$ Binary coverage status of RTP $R_{i}$ by sector $j$ at site $k$

$R_{i-j-k}^{s e r v} \quad$ Binary service status of RTP $R_{i}$ by sector $j$ at site $k$

$r_{\text {overheads }}$ DL subframe overheads

$S \quad$ Set of site locations

$s \quad$ Solution state

$S_{i}^{C A P E X} \quad$ Capital expenditure of site $S_{i}$ 
$S_{i}^{O P E X} \quad$ Operational expenditure of site $S_{i}$

$T \quad$ Annealing temperature

$t_{f r} \quad$ Frame duration

$w_{i} \quad$ Weight of objective $E_{i}$

$z_{k} \quad$ Binary operational status of site $S_{k}$

$z_{j-k} \quad$ Binary operational status of sector $B_{j-k}$ at site $S_{k}$

$\mathbf{R}_{\mathrm{s}} \quad$ The centre of mass 


\section{Chapter 1}

\section{Introduction}

In recent years there has been a tremendous increase in mobile data traffic growth, which has been fueled by the launch of High-Speed Packet Access (HSPA), introduction of flat-rate tariffs, and emergence of smart phones. For example, the Telenor group in Denmark have shown a massive growth of traffic volume with more than $300 \%$ annually, and they forecast that traffic volume will increase exponentially with an average annual increase of $70 \%$ up until 2015, i.e. a total traffic increase factor of 20-25 from 2009 to 2015 [54]. This trend in the growth of mobile data can be attributed to the fact that the adoption of mobile broadband data services is still in its infancy and as such, the long term growth rate is expected to become more moderate. The high expected increase in data traffic volume imposes a great challenge to the service providers all over the world to satisfy the requirements of data services.

Another problem arising with the growth of traffic volume in urban areas is the socalled digital divide. The digital divide was defined as the unequal access to Information and Communication Technologies (ICTs). Although this inequality usually applies to differences between countries (the international digital divide), e.g. comparing developed and developing countries or regions, some analysts also describe unequal access as applying within countries (the domestic digital divide) and most importantly the divide between rural and urban, well educated or poorly educated populations or poor and rich citizens [39]. Another report [124] suggests that financial factors contribute significantly to the ICT deficit for lower socio-economic groups within our communities and in less economically developed countries. Deploying traditional wired 
infrastructures in remote, sparsely populated areas has been commercially infeasible and has created a huge financial barrier to getting these communities connected to the Internet. A community's inability to use ICT effectively also contributes to the digital divide.

Experts also suggest that although third generation systems dominate mobile technology currently, they alone will prove unable to meet increasing demand, and a mix of technologies and business models is needed to exploit the mobile information and entertainment markets, reported by Peter Rysavy, President of Rysavy Research. In order to meet the ever growing demand in mobile broadband and to bridge the digital divide a new technology, namely WiMAX, was introduced in 2004. Together with the appearance of the new standard the WiMAX Forum was created with support of leading companies in the telecommunication industry, describing WiMAX as 'a standards-based technology enabling the delivery of last mile wireless broadband access as an alternative to cable and DSL'. The aim of the WiMAX Forum is to create interoperability of devices to encourage competition in the market place. Ultimately, this competition will drive down prices and make access more affordable. Key strengths of WiMAX include:

- Superior performance, made possible by the adaptation of OFDMA multiplexing, which gives WiMAX a performance edge in delivering IP data services compared to $3 \mathrm{G}$ technologies.

- Flexibility, which allows service providers to support multiple usage models, including fixed and mobile access, over the same WiMAX infrastructure and to operate their networks in multiple spectrum bands.

- Advanced IP-based architecture, which includes IMS support to facilitate a rapid, low cost, rollout of new applications and of interworking with $3 \mathrm{G}$ and other technologies. 
- Attractive economics, driven by a standards-based approach, cost-effective infrastructure, mass adoption of low-cost subscriber units, and attractive IPR royalties.

WiMAX technology which is based on next-generation all-IP core networks, offers low latency, advanced security, QoS (Quality of Service), and worldwide roaming capabilities allows to bring broadband services to communities traditionally underserved by older high speed Internet services. Because WiMAX is a long range, highly scalable system, it enables service providers to reach customers even in areas with no existing cable or telephone infrastructure. WiMAX also allows the service provider community to offer "triple play" (voice, video and data) or "quadruple play" (voice, video data as well as mobile voice and data). While currently, the provision of triple/quadruple play services is achieved with 3 or 4 dissimilar networks: Internet/WANs/LANs for data, PSTN for voice, Cable TV for video and Cellular Networks for mobile experience.

To summarize, WiMAX technology can address challenges associated with development such as backhaul, last mile and large-area coverage access. In particular WiMAX networks:

- offer fixed and mobile wireless broadband access in urban and sub-urban areas where copper based services are not cost effective;

- cover metropolitan areas for mobile data-centric service delivery;

- extend the coverage limitations of public WLAN hotspot zones to citywide coverage;

- bridge the digital divide in rural low-density population areas where provision of other technologies is proved to be challenging.

The telecommunications industry, like any other, encourages the service providers to aim for dominating the market over competitors and most importantly increasing company's profits. In order to achieve commercial success and to get a good financial 
return from the investment in new network technologies such as WiMAX, service operators must make every effort in designing and deploying the most cost-effective networks. Historically, the network designs were developed manually by network engineers. However, the growing complexities and introduction of increasing number of constraints specific to evolving technologies makes manual planning impractical and even infeasible. This leads to an increased interest in the development of the automated planning solutions. A significant amount of research has been dedicated to the development of models and planning solutions for $2 \mathrm{G} / 3 \mathrm{G}$ technologies. Traditionally, wireless network planning includes solving the two tasks of cell planning and frequency planning independently. However, the introduction of features in WiMAX that allow superior performance to other technologies dictates the necessity of developing a new approach for planning WiMAX networks. With this in mind the main contributions of this thesis include:

1. Development of a dimensioning technique for Mobile WiMAX technology (which traditionally is based on the analytical assessment of the cell planning problem to determine the required number of sites). A novel approach is proposed which takes the dimensioning problem to a new level and produces more accurate results in comparison to traditional methods, as well as providing a wider range of results in terms of outcome, in other words not limiting the dimensioning process only to the determination of the required number of sites.

2. Development of a decomposed optimization framework for the WiMAX network planning problem. The novel framework proposed in this thesis subdivides the overall problem into 3 distinct stages consisting of the network dimensioning stage which, besides the primary task of evaluating the financial requirements, produces a good starting network solution with the use of only omni-directional base stations (Stage 1); initial sectorization and configuration of the network (Stage 2); and final network configuration (Stage 3). The proposed framework also solves two fundamental problems, cell planning and frequency planning, 
simultaneously.

3. Evaluating the feasibility of the network plans produced by the optimization framework with an enhanced WiMAX model for the OPNET simulator.

A number of publications have been produced during the course of this research work and are as follows:

1) V. Teterin, S. Hurley, and SM Allen. Optimizing performance of WiMAX networks through automated site selection. In International Conference on Convergence of Telecommunications, Networking and Broadcasting, pages 28-29, 2007.

2) V. Teterin, S. Hurley, and SM Allen. A staged optimization framework for cost optimized WiMAX network design. In Wireless and Mobile Communications, 2008. ICWMC'08. The Fourth International Conference on, pages 185-190. IEEE, 2008. Best paper award.

3) V. Teterin, S. Hurley, and SM Allen. Unified wimax network design with service optimization. In Proceedings of the International Conference on Mobile Technology, Applications, and Systems, Mobility 2008, pages 31-38. ACM, 2008.

4) V. Teterin, S. Hurley, and S.M. Allen. Unified WiMAX network design with service optimisation. International Journal of Autonomous and Adaptive Communications Systems, 4(3):220-236, 2011.

The remainder of the thesis is structured as follows:

Chapter 2 looks at the history of cell planning problem applied to different technologies. Discussion also focuses on the specifications of WiMAX which bring new challenges to the network planning problem, investigates available dimensioning techniques, RF optimization approaches used in second and third generation systems and finally discusses optimization for WiMAX network planning.

Chapter 3 introduces the network model and assumptions used and applied throughout the thesis. Specifically the main focus is on the OFDMA subchannelization, slot 
assignment, power allocation, interference and base station throughput estimation.

Chapter 4 provides insight on the optimization framework proposed in this thesis, in particular it describes the optimization technique based on simulated annealing, network design objectives and key performance indicators used to evaluate the performance of the generated network plans, and finally presents the idea behind the problem decomposition.

Chapter 5 is solely dedicated to Stage 1 of the optimization framework, where are all the assumptions related to SINR calculation, capacity estimation and a set of optimization moves are presented and justified. It also provides the structure of the output which is used as an input for Stage 2.

Chapter 6 presents the subsequent optimization Stages 2 and 3 revealing the objectives, structure of neighbourhood moves, and specifications of each stage.

Chapter 7 outlines the representative network scenario that is used in the thesis and presents the optimization framework results as well as four additional experimental scenarios to emphasise the benefits of each optimization stage.

Chapter 8 provides the evaluation framework with a description of the OPNET simulator capabilities, supported features of WiMAX model, demonstration of the simulation approach and finally provides the results of the simulated network plans.

Chapter 9 consolidates the findings from the preceding chapters and provides suggestions for future development of the presented methods and techniques. 
Chapter 2

\section{Technical background and literature}

\section{review}

\subsection{WiMAX technology}

Worldwide Interoperability for Microwave Access (WiMAX) is a standard of an Institute of Electrical and Electronics Engineers (IEEE) defined as IEEE 802.16. At the same time the WiMAX Forum has been created and backed by hundreds of leading companies in the industry to promote interoperability of equipment supporting stationary, portable and mobile applications, as well as to promote and establish a brand for the technology. The WiMAX Forum has designed and presented WiMAX as a last-mile broadband wireless access alternative to various existing telecommunication technologies providing cable and wireless services. Standards which have been developed by the IEEE 802.16 Working Group include:

- 802.16a [12] - approved in January 2003 defining fixed WiMAX with Line-ofSight (LOS) capability - developed as an alternative to T-carrier and E-carrier (E1/T1) systems for enterprises, and Optical Carrier Level (OC-x) technologies, as well as backhaul for hotspots

- 802.16REVd [11] - approved in July 2004, and later published as 802.16-2004, introduced support for indoor Non-Line-of-Sight (NLOS) topologies through ad- 
ditional radio capabilities such as antenna beam forming and Orthogonal Frequency Division Multiplexing (OFDM) subchannelization - developed as an alternative to Digital Subscriber Line (DSL) technologies for indoor broadband access for residential users

- 802.16e [13] - approved in the early 2005, a variant which introduced support for mobility - portable broadband access for consumers.

- other standards $(\mathrm{i}, \mathrm{j}, \mathrm{h}, \mathrm{m})$ are in progress of development concentrating on multihop relay, improved coexistence mechanisms for license-exempt operation, and advanced air interface with data rates of $100 \mathrm{Mbit} / \mathrm{s}$ mobile and $1 \mathrm{Gbit} / \mathrm{s}$ fixed.

The predicted theoretical reach of WiMAX is a 30-mile coverage radius and achievable data rates of up to $75 \mathrm{Mbps}$. Based on OFDM technology Mobile WiMAX offers scalability in both radio access technology and network architecture, thus it is extremely flexible in network deployment options. It poses several key advantages over the traditional CDMA-based Third Generation (3G) systems [145]:

- Tolerance to Multipath and Self-Interference

- Scalable Channel Bandwidth [11, 13, 148]

- Orthogonal Uplink Multiple Access

- Support for Spectrally-Efficient Time Division Duplex (TDD)

- Frequency-Selective Scheduling [152]

- Fractional Frequency Reuse

- Fine Quality of Service $\mathrm{QoS}$

- Advanced Antenna Technology - Beamforming, Space-Time Code (STC) and Spatial Multiplexing (SM), [19, 130, 59, 60] 
All the advantages over existing broadband solutions and deployment considerations can found in more detail from [117, 117, 72, 145, 8, 94]. In order to emphasise these advantages the WiMAX Forum provides a comparative analysis of Mobile WiMAX with contemporary 3G technologies, Evolution-Data Optimized (EVDO) Rev A and Rev B, and HSDPA HSUPA (HSPA), on the basis of performance characteristics such as throughput and spectral efficiency based on simulations using a common set of parameters, [72, 145]. The results show the distinct advantage of Mobile WiMAX over the 3G CDMA-based enhancements, EVDO and HSPA in both spectral efficiency and channel/sector throughput in both the Downlink (DL) and Uplink (UL) directions.

Despite all the benefits new technology brings, the overall performance of a Mobile WiMAX installation is largely determined by the network layout and its configuration. Among the necessary conditions for designing an efficient WiMAX network are therefore careful coverage planning and optimizing using network design parameters such as base station locations, channel assignment, and BS transmit power allocation. These network planning and optimization tasks are the focus of this thesis. First, however, we provide some technical specifications of WiMAX technology and discuss the issues that need to be considered when planning such a network.

\subsection{Mobile WiMAX technical specifications}

The WiMAX air interface adopts Orthogonal Frequency Division Multiple Access (OFDMA for improved multi-path performance in NLOS environments. Scalable OFDMA (SOFDMA) [148] was introduced in the IEEE 802.16e amendment to support scalable channel bandwidths. This section is dedicated to a brief description of all features integrated into the Mobile WiMAX standard with references provided for the full analysis of each specification. All technical specifications in full can be found in [146]. 


\section{Spectrum and frequency bands}

With spectrum resources for wireless broadband worldwide still being quite disparate in its allocations the WiMAX Forum has introduced several profiles for Mobile WiMAX including various channel bandwidths from $1.25 \mathrm{MHz}$ to $20 \mathrm{MHz}$ and frequency bands ranging from $2300 \mathrm{MHz}$ to $2690 \mathrm{MHz}$ and $3300 \mathrm{MHz}$ to $3800 \mathrm{MHz}$. Another benefit of a WiMAX solution is its ability to operate in licensed and licenseexempt frequency bands. Each country defines and regulates its own set of licensed and unlicensed spectrum which makes WiMAX an attractive option with this sort of flexibility. Intel is an active participant of the WiMAX Forum and provides a comparison of both solutions outlining the advantages, challenges and possible usage scenarios for WiMAX [8].

The Mobile Technical Group (MTG) in the WiMAX Forum defines the mandatory features (ensuring baseline functionality for terminals and base stations) and optional features (different configurations that are either capacity-optimized or coverage optimized) of the standard that are necessary to build a Mobile WiMAX network.

\section{Mandatory features}

OFDMA - is a multiplexing technique that subdivides the bandwidth into multiple frequency sub-carriers. The input data stream is divided into several parallel sub-streams of reduced data rate (thus increased symbol duration) and each sub-stream is modulated and transmitted on a separate orthogonal subcarrier. The increased symbol duration improves the robustness of OFDM to delay spread. Furthermore, the introduction of the cyclic prefix $(\overline{\mathrm{CP}})$ can completely eliminate Inter-Symbol Interference (ISI) as long as the $\mathrm{CP}$ duration is longer than the channel delay spread. The $\mathrm{CP}$ prevents interblock interference and makes the channel appear circular and permits low-complexity frequency domain equalization. OFDM exploits the frequency diversity of the multipath channel by coding and interleaving the information across the sub-carriers prior to transmissions. OFDM modulation can be realized with efficient Inverse Fast Fourier Transform (正FT), which enables a large number of sub-carriers (up to 2048) with low 
complexity. In an OFDM system, resources are available in the time domain by means of OFDM symbols and in the frequency domain by means of sub-carriers. The time and frequency resources can be organized into sub-channels for allocation to individual users. The OFDMA symbol structure can be seen in Section 3.5.2.

Adaptive Modulation and Coding (AMC) - support for QPSK, 16QAM and 64QAM are mandatory in the DL with Mobile WiMAX. In the UL, 64QAM is optional. Both Convolutional Code (CC) and Convolutional Turbo Code (CTC) with variable code rate and repetition coding are supported. Block Turbo Code and Low Density Parity Check Code (LDPC) are supported as optional features.

Hybrid Automatic Repeat Request (HARQ) - provides fast response to packet errors (ACK/NACK signalling) and improves cell edge coverage. Its a combination of high-rate forward error-correcting coding, and ARQ error-control for detectable-butuncorrectable errors. In Hybrid ARQ, a code is used that can perform both forward error correction (FEC) in addition to error detection (ED) (such as Reed-Solomon code, Convolutional code or Turbo code), to correct a subset of all errors while relying on ARQ to correct errors that are uncorrectable using only the redundancy sent in the initial transmission.

Fast Channel Feedback (CQICH) - provides fast channel information feedback to enable the scheduler to choose the appropriate coding and modulation for each allocation.

These features AMC, HARQ and CQICH were introduced with Mobile WiMAX to enhance coverage and capacity for WiMAX in mobile applications.

Media Access Control (MAC) - MAC layer is based on the time-proven DOCSIS standard and can support bursty data traffic with high peak rate demand while simultaneously supporting streaming video and latency-sensitive voice traffic over the same channel. The resource allocated to one terminal by the MAC scheduler can vary from a single time slot to the entire frame, thus providing a very large dynamic range of 
throughput to a specific user terminal at any given time. Furthermore, since the resource allocation information is conveyed in the MAP messages at the beginning of each frame, the scheduler can effectively change the resource allocation on a frameby-frame basis to adapt to the bursty nature of the traffic.

QoS - with features such as AMC which ensure a steady signal strength over increasing distance, MAP-based signalling schemes and flexible time/frequency resource allocation mechanisms (which monitors interference and other detractions to signal), allow Mobile WiMAX to meet QoS requirements for a wide range of data services and applications. The main detractors from good QoS are latency, jitter and packet loss. WiMAX offers a very low latency across the wireless span. The main solution in offering good QoS is to prioritize time sensitive traffic such as VoIP and video. Mobile WiMAX has 5 categories:

- Unsolicited Grant Service (UGS) - VoIP;

- Real-time Packet Service (rtPS) - Streaming audio or video;

- Extended real time Packet Services (ErtPS) - VoIP with activity detection;

- non-real time Packet Services (nrtPS) - FTP;

- Best Effort (BE) - Data transfer, Web browsing etc.

Duplexing - Fixed WiMAX supports both Frequency Division Duplex (FDD) and TDD profiles while Mobile WiMAX is currently only defined for TDD mode. TDD is a technique whereby information is transmitted and received using a common frequency band but at different times. There are arguments in the preference of use for both duplexing mechanisms, but it can be noted that TDD has the potential to offer improved spectral efficiency over FDD in applications with asymmetric downlink/uplink traffic [9]. 
Most of the mandatory features are considered in the proposed optimization framework in some way or another, with exception of HARQ and fast channel feedback which are the dynamic features of a scheduler.

\section{Optional features}

As optional base station deployment configurations are not considered in this work we provide only a list of these features. Full analysis, however, can be found in [73]. These optional features include:

Adaptive Antenna System (AAS) - adaptive antenna systems use beam forming technologies to focus the wireless beam between the base station and the subscriber. This reduces the possibility of interference from other broadcasters as the beam runs directly between the two points [146].

Frequency reuse - traditional reuse conventional cellular deployments used cell frequency reuse factors as high as 7 to mitigate inter cellular Co-Channel Interference (CCI). These deployments assured a minimal spatial separation of 5:1 between the interfering signal and the desired signal but required seven times as much spectrum [73]. With technology such as OFDMA, more aggressive reuse schemes can be employed to improve overall spectrum efficiency. Two common frequency reuse configurations for a multi-cellular deployment with 3-sector base stations are used - reuse of 3 and reuse of 1 , also referred to as universal frequency reuse. With a frequency reuse of 1 the same channel is deployed in each of the three base station sectors.

Multiple in and multiple out (MIMO) - with multiple transmitters and receivers built into the antenna, the transmitter and receiver can coordinate to move to an open frequency if/when interference occurs.

Software defined radio (SDR) - SDR have the flexibility to dynamically shift frequencies to move away from a congested frequency to an open channel. 


\subsection{Network planning and optimization issues}

As WiMAX possess new technologies such as SOFDMA, adaptive modulation and coding schemes, space-time coding and spatial multiplexing, flexible subchannelization, and flexible network deployment, these technologies allow the standard to deliver high data rates and to satisfy the ever growing demand for broadband services. Consequently significant optimization is required for the network design to achieve the desired superior performance.

Traditionally the network planning and optimization of wireless networks includes the following stages:

1. Network survey

2. Network dimensioning

3. RF optimization

4. Radio resource allocation optimization

5. Scheduling optimization

This section describes each stage in detail and provides an analysis of related work for WiMAX technology and other alternatives.

\subsubsection{Network survey}

When a company decides to build and operate a wireless broadband network it has several important issues to consider in terms of how to proceed. After commercial and residential market analysis is complete which includes: definition of the service area, the projected number of customers, their distribution, spectrum availability, growth rate, and system usage, and the network interconnect agreement, numbering, and routing policy for inter-network access and roaming, an operator must conduct a thorough 
engineering site survey of the area to be served. The data obtained from the survey will aid in the development of a preliminary network design. The interference issues and risks need to be studied very carefully. The quality of this survey can provide an invaluable insight into current or potential interference issues.

A site survey is very important as it provides to the operator the necessary information for a complete WiMAX network blueprint, which helps to minimize the unknowns regarding the network, minimize costly mistakes prior to construction, and provide a vision of how the network will behave once it is deployed.

Variables to be considered in a wireless network by experienced network engineers may include [7]:

- The impact of the fresnel zone on signal propagation

- The difference between passive gain and powered amplification, and when use of each is appropriate

- The orchestration of sector, space, frequency and polar diversification(s)

- The use of a Front to Back ratio

- How many channels are available for use and where are potential interferers located

- The $+/-3 \mathrm{~dB}$ rule and how it influences the link budget

- How and why application must drive the hardware

All these engineering considerations are site-specific and represent only a fraction of the issues that must be observed during the survey. More information obtained from the survey translates into higher engineering and performance accuracy, which minimizes the total cost of network deployment. 


\subsubsection{Network dimensioning}

Network dimensioning activity is designed to evaluate and estimate the main infrastructure requirements required to provide coverage, capacity and quality of service. This can estimate the capital and operational costs for designing a WiMAX broadband network with specified characteristics. As is widely presented in the literature, specifically in Wideband Code Division Multiple Access (WCDMA) network dimensioning [80, 30, 95, 21, 116], essentially the sole purpose of dimensioning is to provide a quick count of sites in accordance with some theoretical approach, which provides a basis for the next step of network planning. The main dimensioning process is performed after the network survey has been done and all the technical requirements and specifications of the network have been identified. At this point accuracy is crucial and hence care should be taken to achieve it since if the predictions from dimensioning turn out to be inaccurate for the deployment of the network it is very costly to reposition incorrectly located sites afterwards. The results of this stage are used as input in the preliminary network design, which includes the preliminary distribution of base station equipment to service areas and preliminary BS configuration (i.e. sectorization, air-interface parametrization). The results of the network dimensioning activity have a great impact on the business case of a WiMAX network investment. It essentially assists in the business planning and budget planning process and allows an investor to identify the best proposed strategy and understand the likely return on investment. The benefit of dimensioning is its ability to provide a diversity of strategies that address the crucial issues such as the reduction of Capital Expenditure (CapEX), Operating Expenditure (OpEX) and network complexity, and improvement of time-to-market deployment.

A number of papers has been published on the dimensioning techniques for $3 \mathrm{G}$ networks $[21,110,10,82,99]$. Some methods include only coverage dimensioning, others a joint estimation with capacity dimensioning based on the load factor equations and providing lower bounds on the sites for selection [21]. Others provide a comparison 
between the results obtained from the dimensioning process and comprehensive system level simulation stating that the results only vary in the range of $0-15 \%$ [110]. However, there appears to be only two studies [10, 82] that consider the network dimensioning of WiMAX technology, both applying similar approach.

Traditionally the process of dimensioning is based on the number of assumptions (such as homogeneous morphology and ideal site distribution) and involves a sequence of steps, which serve different requirements such as capacity or coverage estimations [10]. First, the input parameters are defined consisting of the business plan, the assets, and the Key Performance Indicators (KPI). Next, the coverage analysis is performed which provides an estimation of the service areas and the required number of sites. Normally at this stage a simplified representation of base stations is considered, with three sectors providing a 360 degrees footprint. Following the coverage analysis, the capacity estimation checks for sufficient capacity resources as dictated by customer numbers and services profiles. Therefore this step estimates the required number of sectors to achieve this capacity. The final step is a joint analysis which aims to estimate number of BSs and sectors to satisfy both coverage and capacity. Coverage, capacity, and joint analysis may be revised several times before providing the final dimensioned network.

As has been noted already the output of this activity also provides input to the core network engineering team to design the network topology as well as to decide the number of backhaul links required for the initial launch of the network. Since the result of network dimensioning significantly impacts the network reliability and initial implementation cost, each parameter that is used in the dimensioning process must be thoroughly considered.

The advantages of WiMAX technology which offer great flexibility and scalability, however present more design challenges. The challenges in WiMAX network dimensioning and planning compared to conventional $2 \mathrm{G} / 3 \mathrm{G}$ cellular network planning involve evaluating the implication of each of the optional and mandatory features (sec- 
tion 2.2 and then consider the best combination of the supported features to suite the operator's business model. Once again the traditional approach in dimensioning networks [10, 82] incorporates two stages - coverage and capacity analysis to identify the required number of base stations and sectors satisfying the business model.

Coverage analysis: the objective is to ensure a sufficient number of base stations to satisfy the required coverage level. In [10] the authors identify this by dividing the service area by the cell footprint to produce the necessary number of BS. The cell footprint calculation depends on the specifications of the equipment and estimation of the maximum system range. This commonly adopted approach is based on the link budget which sums the losses and gains in signal strength as it travels through different components in the path between transmitter and receiver. This gives the limit of the cell range for different configurations in order to maintain a balanced uplink and downlink. Generally the link budget represents the whole system as an average in terms of the derived cell radius, providing the engineering guidelines to begin cell layout and design. Also the hexagonal representation of a cell is often used in the dimensioning to simplify the calculations [10].

Capacity analysis: involves assessing the traffic demand for different service requirements considering the activity factor, overbooking/contention ratio, and TDD ratio for the uplink and downlink, in order to ensure sufficient air-interface capacity (throughput) for a wide range of services. Therefore the purpose of capacity dimensioning is to translate the capacity into a number of sectors, which are to be distributed within the estimated number of sites. With the support of an adaptive modulation and coding scheme, the calculation method widely adopted is a ring structure [82], where the throughput calculation is based on the area of each modulation scheme. Thus the average operating sector throughput is normally adopted, which determines how many subscribers can be served in a sector. Defining this average sector throughput is a complex task as it involves interpretation of a wide range of parameters. Another option which can be employed is the usage of sector throughput provided as a recommenda- 
tion by the system vendor, however it can vary significantly depending on the deployment scenario and not as accurate. A more accurate estimation is through extensive RF planning simulations and statistical processing that would result in a $\mathrm{PHY}$ mode regions map and give the average cell throughput. Another way employs the assumption traditionally considered, the average sector throughput, by using of the mostly used modulation and coding scheme (16-QAM 1/2) [82]. The final step for this approach is to combine the results from both analysis into the optimum BS configuration. This includes three possibilities: Balanced network, Coverage-limited network, Capacitylimited network [10].

The major drawback of these approaches is a lack of accuracy. Both analysis methods described in this section are based on the averaged performance of the cells: for coverage estimation based on the link budget and averaged cell radius, and for capacity on the analytical estimation of the averaged cell capacity. An interference estimation with this approach is also unreliable. Another weakness of this method is that there is no account for the signalling overheads in the throughput calculation which makes the capacity overestimated. These predictions of the required infrastructure based on empirical models can differ significantly with the number of BS derived from radio planning bringing the investor/service provider to a misleading conclusion in terms of the financial commitment and potential return on the planned network. This in turn has a negative impact on the following stage of the physical planning of the network. In this thesis we propose a completely different and novel approach which incorporates all the specifics of WiMAX technology, with accurate interference and capacity estimation methods. The resulting output of this approach not only provides the count on the required number of sites but also optimal positions for their locations and the regions which have to be serviced by the sites. This approach is more accurate and faster and can be used as a dimensioning tool in itself and at the same time produce initial solutions for the following configuration and optimization stages (described in Section 5). 


\subsubsection{RF optimization}

It is known that the wireless radio network planning problem belongs to the class of NP-hard problem [52, 25, 103]. Thus, a theoretical optimum cannot be found in polynomial time. This also implies that the solution time can grow quickly as the number of variables grows. Meta-heuristic techniques have become a common approach to solve this kind of problem in traditional cellular networks [25]. With no guarantee in finding an optimal solution, they have proven to perform very well for practical problems with large number of variables. The utilization of optimization techniques for wireless network planning is not new. However, with the emergence of new technologies providing wireless access it is extremely important to assess and take into account new factors affecting the planning process.

RF optimization is the key step in wireless network planning. The aim is to produce a network solution which satisfies the trade-off between the given objectives, such as maximizing coverage, service and minimizing capital and operational costs. Traditionally RF optimization consists of site location selections, frequency planning and BS configuration which include antenna orientation selection (azimuth and tilt), BS radiation power, antenna type and other technology-specific parameters. In order to evaluate the performance of the network plan a static snapshot-based simulation is normally used due to the high computational load of dynamic simulation [29].

A number of optimization models have been introduced for wireless network planning. These models could be solved by commercial solvers, such as CPLEX. However, in practical instances the large size of the problems makes the use of heuristic procedures more popular. An initial approach to wireless network planning was to partition the network area into equally sized hexagons [126]. Later this approach deemed to be unusable because of the oversimplified approximations and assumptions incorporated, which were unsuitable for practical planning. The massive growth and demand in wireless communications led to the necessity of network expansions and the requirement for engineers to plan frequency spectrum more thoroughly. These difficulties of operat- 
ing with large and complex planning problems and scarce radio resources required the need to develop effective automatic design algorithms based on mathematical optimization techniques. In the early 1980s the frequency assignment problem was recognized as equivalent to the Graph Coloring Problem which results in assigning a frequency to each vertex of a graph so that adjacent vertices receive different colors and the number of colors is minimized [14]. This resulted in the development of heuristic algorithms to obtain optimal colorings to solve the frequency assignment problem. The popularity of mathematical optimization techniques has inspired the development of more accurate representations of network instances and later hexagonal cells were replaced with the demand nodes introduced by Tutschku [133], and now referred to as test points (TP).

After the appearance of the TP model, more accurate models and effective optimization algorithms have emerged for the cell placement problem, finding suitable site positions and base station parameters for the coverage of a wireless network [27]. A number of models defined by associating suitable decision variables with the physical and radioelectrical antenna parameters (candidate locations, power values, activation statuses, transmission frequencies, service, and coverage requirements ) and solving coverage and capacity planning problems have been developed and applied to the design of Global System for Mobile Communications (GSM) [106, 140, 83, 143, 139, 22, 144] and Universal Mobile Telecommunications System (UMTS) [26, 51, 25, 142, 137, 141, 24]. Authors in [138] have provided a detailed survey of proposed approaches for network design, charting the evolution of models and techniques for the automatic planning of cellular wireless services.

Discrete programming models for wireless network optimization have been proposed in [52] with the aim to select an optimal subset of site locations from a defined set. These are formulated as integer linear programming (ILP) problems and solved by combinatorial optimization techniques, however, this approach is too generalized and does not take account of some economic and technical constraints.

When planning the wireless network, coverage and capacity problems should be con- 
sidered simultaneously, as both depend on common network configurations. However, the traditional approach in handling large-scale network problems normally appeals to a method involving decomposition of the problem to separate coverage and capacity planning [133]. This approach involves an initial placement and configuration of base stations in order to satisfy the coverage level, followed by solving the frequency assignment problem to meet the required capacity level. In order to satisfy the growth in capacity demand in the future an operator can install additional antennas or assign additional transmission frequencies [118]. In [149, 150, 151] a mathematical model for UMTS radio network planning is presented, and different single-objective optimization strategies are investigated. The Base Station (BS) location problem is modelled as a simplified problem, and parameter tuning for different meta-heuristics is presented.

With the development and maturity of technologies new optimization challenges have emerged which are related to radio resource allocation, in particular power control and frequency resource allocation to users. A combined power control and base station placement was proposed for UMTS networks in [25], where the traffic demand and power control were addressed simultaneously to obtain a successful network plan. The problem was formulated as a Mixed Integer Problem (MIP) constrained by a set of BS candidate sites, which are selected based on the accessibility of the locations. This was followed by [76], where equal cell loading was considered as a key factor for an efficient Third Generation (3G) network based on a four CDMA cell experiment. These methods were further expanded to include IEEE 802.11 based technologies in [53]. The network design problem is formulated into two optimization problems, first, the facility location problem which deals with the Access Points (APs) locations, and second, the graph coloring problem which distributes frequency channels in certain pattern to reduce the interference between the reused frequency bands.

WiMAX is often compared to Wireless Fidelity (Wi-Fi) technology, when in reality both standards are different in many respects. The Wi-Fi MAC layer uses contention access. This causes users to compete for data throughput to the access point. Wi-Fi 
also has problems with distance, interference, and throughput and therefore triple play (voice, data, video) technologies cannot be hosted on traditional Wi-Fi. In contrast, 802.16 uses a scheduling algorithm. This algorithm allows the user to compete once for the access point. This gives WiMAX inherent advantages in throughput, latency, spectral efficiency, and advanced antenna support.

One of the challenges associated with planning WiMAX networks is financial. Operators have to identify requirements related to the market segment targeted, types of services to provide, spectrum available and the topography of the service area. There is no single solution for success, thus operators need to make a thorough technical analysis including a viable business model and build a network in the most efficient way to realise the goals. The main challenges in WiMAX network planning in comparison to current $2 \mathrm{G}$ and $3 \mathrm{G}$ technologies however is consideration of all technical features which the standard possesses:

- OFDM - the implementation of various AMC schemes, the radio resource allocation, scheduling and other features, not only increase the throughput of base stations but also radically changes the methodology of interference and capacity evaluation

- channel bandwidths - with flexible channelization ranging from 1.25 to $20 \mathrm{MHz}$, the channel offers up to four times more bandwidth than channels of current systems, as well as different frequency reuse schemes including more aggressive fractional reuse of bandwidth.

Wireless networks have grown rapidly as a result of increasing the multi-service traffic demands in terms of data rate, QoS and bandwidth requirements. Thus, the network cost increases according to these demands. In order to meet these demands, the service provider has to invest in developing new methodologies, which maintain the balance between the service quality and the network cost, whilst considering several constraints and influential factors that restrict the outcome of these methodologies. 
There is a limited number of studies that consider the detailed complexity of Mobile WiMAX factors for optimization. In [100], for example, authors present a heuristic clustering algorithm for minimum cost wireless broadband overlay network deployment based on techniques derived from the set covering problem, capacitated facility location and LP-rounding algorithms. However, a very simplified approach is presented without consideration of an accurate interference calculation. A very thorough economic investigation of the problem is presented in [75], but only a few physical constraints are taken into account, such as the size of the cells and the number of radio channels used. In [36] the authors investigate a mesh topology design employing 802.16a radio access in order to provide a backhaul service for a $3 \mathrm{G}$ network. In [129] different optimization algorithms including Tabu search are compared for automatic cell planning in WiMAX networks. However, the authors mainly focus on the economic problem without implementation of all WiMAX-specific base station configurations.

In [46] an optimization model for WiMAX network planning is presented. The physical and radio-electrical parameters such as base stations locations, emission powers, transmission frequencies, and service areas are identified as the main decision variables of a mixed-integer linear programme (MILP). The network quality is evaluated by basic performance indicators such as network coverage and capacity. The WiMAX Network Planning Problem (WNPP) is stated as finding positions and emission powers of the BSs in order to maximize the overall profit. CPLEX is used as a tool to solve the MILP. However, once again the model is simplified and does not consider OFDMA structurization of time/frequency resources, slot based power control and interference calculations. Moreover, a standard sectorization approach is employed with 3-sectored base stations, two types of antennas are considered - omnidirectional antenna and directive with an $120^{\circ}$ pattern, as well as three $7 \mathrm{MHz}$ channels used for frequency assignment.

Authors in [70] present a multi-objective optimization framework for the IEEE 802.16e 
standard. The meta-heuristic algorithm used is Tabu search where QoS, coverage, interference and costs are the criteria to the BS location problem in a Mobile WiMAX deployment. The results produced by the proposed methodology are evaluated using a network simulation tool - Forsk Atoll. First the authors identify the WiMAX-specific physical factors that affect network simulation, planning and optimization processes. Second they simplify the problem and translate it into a formal optimization routine with consideration of economic factors. The main emphasis of the work is on the optimal BS location, i.e. finding optimal site locations for the BS deployment. Most of the BS configurations have been omitted from the optimization procedure, with placement of 3 -sectored base stations with fixed azimuths and 0 degrees tilting. The network instance comprises 20 potential site locations and 320 mobile stations for the service. The planning tool comprises a financial model which incorporates costs (CapEx and OpEx expenditures) and revenue of a Mobile WiMAX network. The problem is formulated to select candidate sites from each region to install BSs such that the traffic capacity and the number of covered users are maximized with the lowest installation cost. The optimization objective function includes aggregation of all costs and penalty functions applied to every user according to criteria such as coverage, interference or QoS indicators. The framework employs a $1 \times 3 \times 1$ frequency reuse scheme, i.e. a frequency reuse factor of 1 and three sectors per site. The interference model does not consider the specifics of OFDM frame structure with subcarrier collisions, and therefore is a simplified generalized model. The optimization problem is known as p-median ${ }^{1}$ where only the BS location is considered as the decision variable.

In [18], a planning method is proposed for wireless networks which are based on OFDMA technology. The authors consider a heterogeneous traffic pattern with a wide variety of service demands in terms of data rate and QoS. The planning problem is formulated as a non-linear multi-objective optimization problem, where the optimum solution should not dominate the throughput of one BS over any other. The aim of the

\footnotetext{
${ }^{1}$ The p-median problems constitute searching $p$ number of locations each time regardless of how distant the sites are.
} 
work is to provide equal loading of all cells. The proposed method seeks an optimal solution by tackling the problems of BS positioning and resource allocation simultaneously. A Pareto-Metaheuristic (PMH) is adopted which is claimed to achieve a balanced throughput over all cells as well as minimizing the number of the installed BSs targeting a certain service outage probability. The problem is decomposed into two problems: the facility location problem and resource allocation (subcarriers and power). However a relatively small number of users are used in experiment (1300) and are randomly located, also the cost model is simplistic as it only considers minimization of network cost by minimizing the number of base stations selected. 
Chapter 3

\section{Optimization \& network model}

The aim of the wireless network planning is to find an efficient network design of a WiMAX MAN with regards to users' service level, coverage and profitability of the network, given a set of BS locations, sector equipment configurations and subchannelization schemes for different channel sizes. Although the approach and model is generic and has been widely used for planning wireless mobile networks based on $2 \mathrm{G}$ and $3 \mathrm{G}$ standards [83] in our case we focus on and introduce new aspects of the model originating from OFDMA structure of IEEE 802.16e networks. Namely, the standard model was amended by incorporating the following new elements: the propagation model based on ray-tracing technique with the use of Rapid Pipeline Development tool (Section 3.2); subchannelization procedure inherent to the WiMAX OFDMA [13] (Sections 3.5.2 and 3.5.3), formulation of the slot assignment problem (Section 3.5.4), formulation of the collision-based interference calculation (Section 3.6), throughput estimation with consideration of transmission overheads (Sections 3.7 and 3.8).

Unlike $2 \mathrm{G}$ and $3 \mathrm{G}$ mobile standards, the channel assignment in networks adopting OFDMA is adjusted over time to take advantage of multiuser channels and traffic diversity. This makes the problem more computationally demanding but utilizing realtime system information leads to higher spectrum efficiency of the system. Therefore the planning stage should take into account the dynamic nature of the network and jointly solve two fundamental problems (cell planning and frequency planning) which traditionally have been solved in sequence [97]. 
In this chapter we will formulate the problem and model for Automatic Cell Planning $(\overline{\mathrm{ACP}}$ and Automatic Frequency Planning (AFP as separate tasks, in the way they are considered traditionally in wireless network planning, and then introduce new combined strategy which allows for finding more efficient solutions.

\subsection{System Model}

The main idea of wireless network planning is finding the configuration of a network which will maximize the performance of that network, as well as satisfy all objectives and constraints. This involves: the initial selection of potential site locations for base station (BS) deployment, identification of required number of sectors (sectorization) and appropriate equipment for each active site, i.e. antennas, for provision of efficient coverage and satisfaction of traffic demand from users in the covered area.

The network is modelled using the following components:

\section{Reception Test Points}

The area where network coverage and service are desired, is modelled by a set of grid points, $R$, and referred to as Reception Test Point $(\underline{\mathrm{RTP}})$. Each point in the grid represents one or more users (further mentioning of RTP will correspond to a user and vice versa), and will have associated parameters:

- traffic demand in Kbps, $\tau$, which is determined by the DL bit-rate

- geographical position in Cartesian coordinates $\mathrm{x}, \mathrm{y}, \mathrm{z}$

- annual revenue which is generated by the network provider if the user is serviced

Therefore any RTP can be presented as a function of following parameters:

$$
R_{i}=\{x, y, z, \tau, r e v\}, R_{i} \in R
$$


where $x, y, z$ are the coordinates of RTP $R_{i}$ in the network grid, $z$ is elevation above the sea level measured in meters, $\tau$ is the traffic demand from the user located at $R_{i}$ and finally rev is the predicted revenue when service is provided for the user.

The equipment for all the users' terminals is assumed to be identical with a $0 \mathrm{~dB}$ antenna gain.

\section{Potential Site Locations}

A set of potential site locations for base station placement is denoted as $S$. We consider the problem where all the locations are predefined with a fixed number of candidates $S=\left\{S_{1}, S_{2}, \ldots, S_{\max }\right\}$ and therefore the choice and maximum number of the locations are not part of the network design process considered in the thesis. These locations are selected by service providers prior to planning taking place. The choice is governed by several factors such as cost of BS installation, annual maintenance (these are considered in the network profitability predictions), electromagnetic compatibility with the surrounding infrastructures and equipment (governed by EMC regulatory body), security, geographical location, planning permission and the most important - propagation potential of the site. In urban areas the BSs are often deployed on the roof tops of the buildings to cover the maximum possible area. The propagation predictions form pathloss (or attenuation) figures between potential site locations and RTPs, measured in $\mathrm{dB}$. These losses do not include factors such as antenna characteristics - feeder cable, jumper, and connector losses, loss/gain due to mast head amplifier, antenna configuration - height, azimuth and tilt, and user equipment characteristics. Given the specific equipment selected at a site these characteristics can be found from manufacture and then used in the design process.

All site locations are associated with the following parameters:

$$
S_{i}=\{x, y, z, Q, C A P E X, O P E X\}, S_{i} \in S
$$

where $x, y, z$ are coordinates in the network grid, $Q_{S_{i}-R}$ is the path-loss prediction matrix represented as a set of signal attenuations from the site $S_{i}$ to all test points from 
the set $R, C A P E X$ and $O P E X$ are the capital and operational expenditures respectively, related to the installation and maintenance costs of the site. The installation costs are one-off payments, operational costs are charged on the yearly basis.

Each site has a binary operational status:

$$
z_{k}=\left\{\begin{array}{l}
1-\text { if site } k \text { is active } \\
0 \text {-otherwise }
\end{array}\right.
$$

where an active status implies that a BS is deployed at a particular site, i.e. operational.

\section{Sectors}

Having determined a set of site locations for deployment of base stations (decision variables in the optimization framework), the next step is to identify a set of sector/antenna equipment for each BS, $B$, essential for effective coverage and service. The maximum number of sectors at a BS is limited by the need to maintain an acceptable level of inter-cell interference and also any hardware restrictions.

Sectors have a number of operational parameters to be configured which are decision variables in the optimization framework. These include:

$S$ - site location where the sector is installed.

$A$ - type of antenna. These range from omni directional which radiates power in a 360 degrees horizontal plane, to a number of directional with 30, 60, 90 and 120 degrees radiation patterns. Designed for urban environments with high cell density, the antenna equipment must deliver consistent reliable data streams to customer devices, deliver equal RF power across the entire cell and in areas of high customer concentration reduce cell to cell interference.

$G$ - antenna gain in $\mathrm{dBi}$, varies with different types of antenna. This specifies the intensity of an antenna in the direction of main beam.

$L_{h o r}$ - horizontal losses of antenna in $\mathrm{dBi}$, varies with different types of radiation patterns. Defined by antenna manufacturer's horizontal diagram. In this diagram the angle 
between the half-power $(-3 \mathrm{~dB})$ points of the main lobe, when referenced to the peak effective radiated power of the main lobe, is a beamwidth and is expressed in degrees. $L_{v e r t}$ - vertical losses of antenna in $\mathrm{dBi}$, varies with different types of radiation patterns. Defined by antenna manufacturer's vertical diagram. Also can be specified with beamwidth (in degrees).

$P$ - BS transmitting power, a set of 10 levels is defined ranging from 27 to $45 \mathrm{dBm}$. In this work it is assumed that if a power level is chosen for the sector then all the users within the cell will be sent a signal at the same power level irrespective of the channel conditions.

$\alpha$ - tilt angle of antenna, from 0 to 15 degrees. Tilt is the angle of the main beam of antenna below the horizontal plane. The main goal of tilting the antenna is to reduce inter-cell interference in order to improve the SINR for users at the serving cell which in turn increases the cell capacity. There are two ways of tilting with current technologies - mechanical and electrical. Mechanical tilting is performed at the initial stage of BS installation. Electrical tilting is more dynamic and can be performed remotely by the operator. The electrical method does not make any physical changes to a tilt, but adjusting the radiating currents in the antenna elements to lower the beam. However the electrical tilting is quite limited due to EMC regulations.

$\beta$ - azimuth angle of antenna, ranging from 0 to 360 degrees. In this work azimuth is the horizontal angle between $\mathrm{x}$-axis of antenna and the main lobe direction. As tilt parameter, azimuth has a vast influence on coverage and overlap levels. Reducing cell overlap with azimuth adjustment in some cases can be achieved without reduction of coverage. However less overlap means less inter-cell interference and power consumption, which also increases cell capacity. Unlike with tilting, azimuth is mostly adjusted mechanically and not as flexible.

$C A P E X$ - antenna cost. As with the costs of site installation and maintenance, the antenna cost plays a role in profitability estimation. 
PermBase - is an integer ranging from 0 to 31, which identifies the particular BS and is specified by the MAC layer. The role of PermBase is fully described in Section 3.5 .2

Given all these parameters and variables a sector can be described by

$$
\begin{gathered}
B_{i}=\left\{S_{j}, A, G, L_{\text {hor }}, L_{\text {vert }}, P, \alpha, \beta, C A P E X, \text { PermBase }\right\} \\
S_{j} \in S, z_{j}=1
\end{gathered}
$$

Each sector has a binary operational status:

$$
b_{i-j}=\left\{\begin{array}{l}
1-i f \text { sector } i \text { at site } j \text { is active } \\
0 \text {-otherwise }
\end{array}\right.
$$

If a sector $j$ is installed at a site $S_{k}$ the transmit power is selected from the predefined set $B_{j-k}^{P}$.

Assumption 3.1.1. The set of available transmitting powers is set to be the same for all the base stations in the network.

\subsubsection{Calculating horizontal and vertical loss}

As previously mentioned the antenna radiation pattern is represented by diagrams of loss figures for the horizontal and vertical planes, measured in $\mathrm{dBi}$. Examples of such diagrams are illustrated in Figure 3.1 for a directional antenna and in Figure 3.2 for an omni directional antenna pattern. An example of the actual loss table for a directional antenna pattern is presented in Table 3.1 . The cumulative directional loss from a given antenna to a user in the network is determined by the horizontal and vertical angles between the main beam of the antenna radiation and a line connecting the user and antenna location.

The illustration of the vertical loss calculation scheme is depicted in Figure 3.3 and Figure 3.4. The parameters which are considered in the calculation are: 

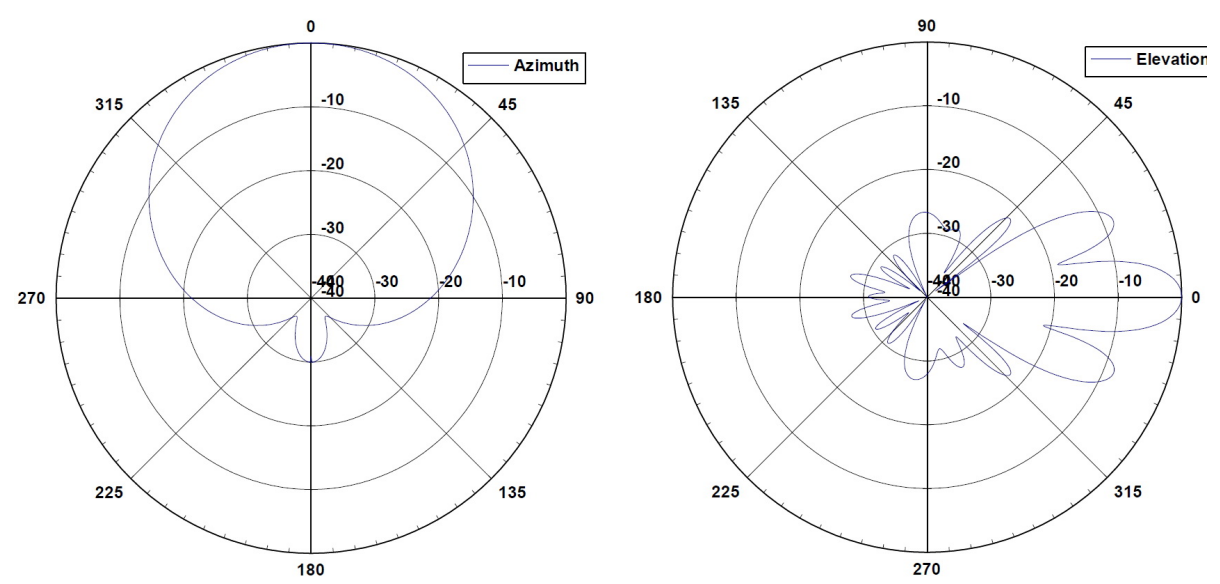

Figure 3.1: Horizontal and vertical planes of 60 degrees directional antenna pattern.
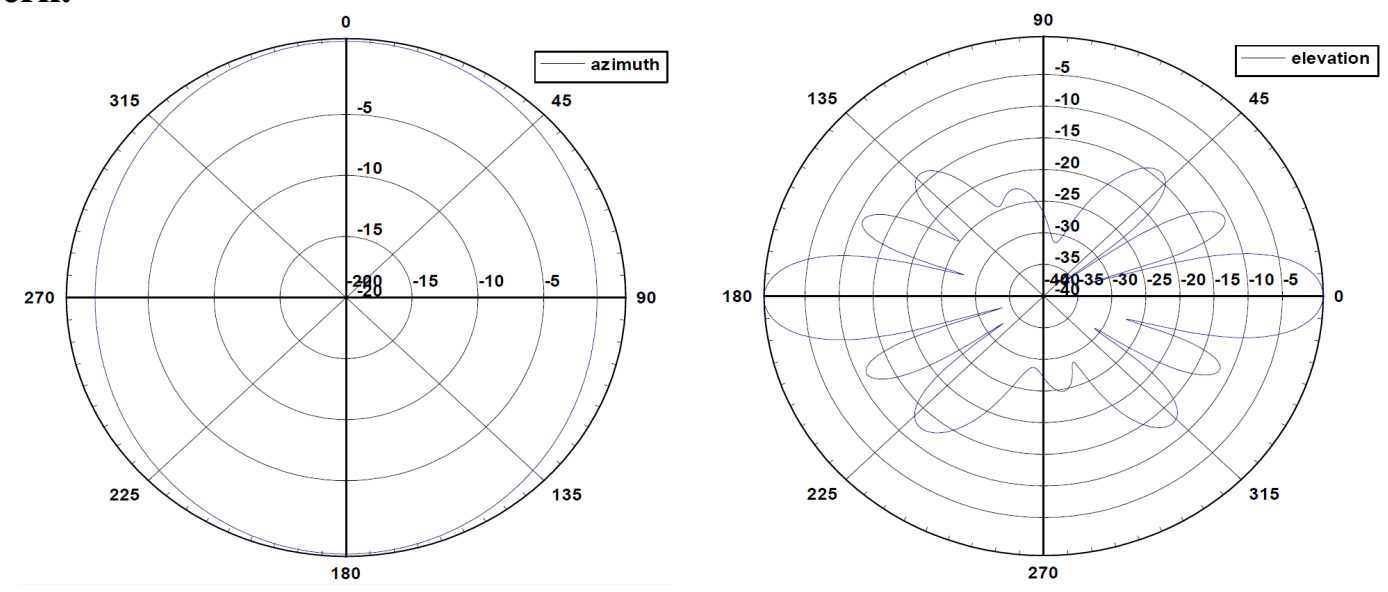

Figure 3.2: Horizontal and vertical planes of omni-directional antenna pattern

- antenna tilt $\alpha$

- antenna azimuth $\beta$

- $\mathrm{x}, \mathrm{y}, \mathrm{z}$ coordinates of site where antenna is installed and RTP where the user is located

In order to find the vertical loss of the antenna at a user location the angle $\theta$ needs to be calculated. This angle indicates how much the horizontal plane of the antenna has 
to be turned so that antenna plane (main lobe axis) aligns with the RTP. This can be expressed as follows:

$$
\begin{gathered}
\text { Dist }_{\text {vert }}=S_{j}^{z}-R_{i}^{z} \\
\text { Dist }_{\text {hor }}=\sqrt{\left(S_{j}^{x}-R_{i}^{x}\right)^{2}+\left(S_{j}^{y}-R_{i}^{y}\right)^{2}} \\
\psi=\beta-\arctan \left(\frac{S_{j}^{y}-R_{i}^{y}}{S_{j}^{x}-R_{i}^{x}}\right) \\
\text { Dist }{ }_{\text {hor_proj }}=\text { Dist }_{\text {hor }} / \cos (\psi) \\
\theta=\text { arctan }\left(\frac{\text { Dist }}{\text { Dist }_{\text {vert_proj }}}\right)-\alpha
\end{gathered}
$$

The next component of the antenna pattern loss calculation is the horizontal angle, and is calculated using the $x, y$ coordinates of the RTP and the site at which antenna is deployed. It is illustrated in the top projection of Figure 3.3. First, the angle between the $\mathrm{x}$-axis and RTP is found - anticlockwise. Then, the required angle is simply the difference between main lobe of antenna and RTP axis, and is expressed as $\psi$. 


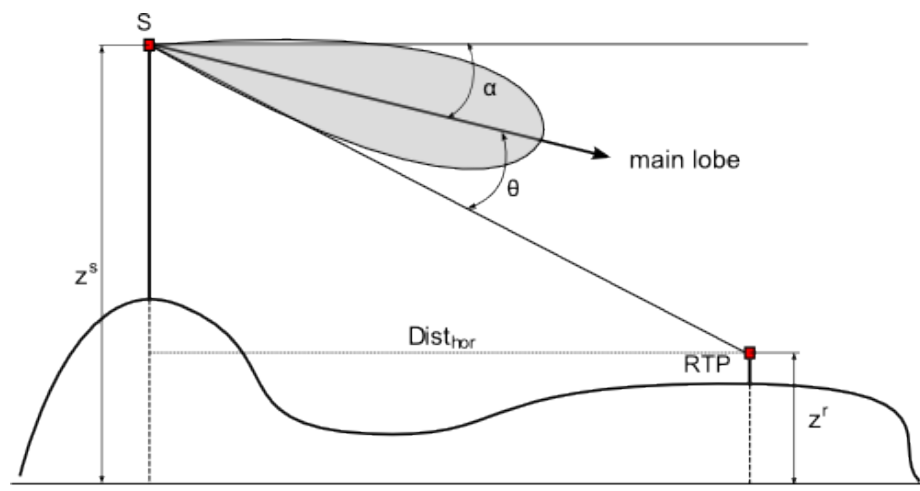

Side projection

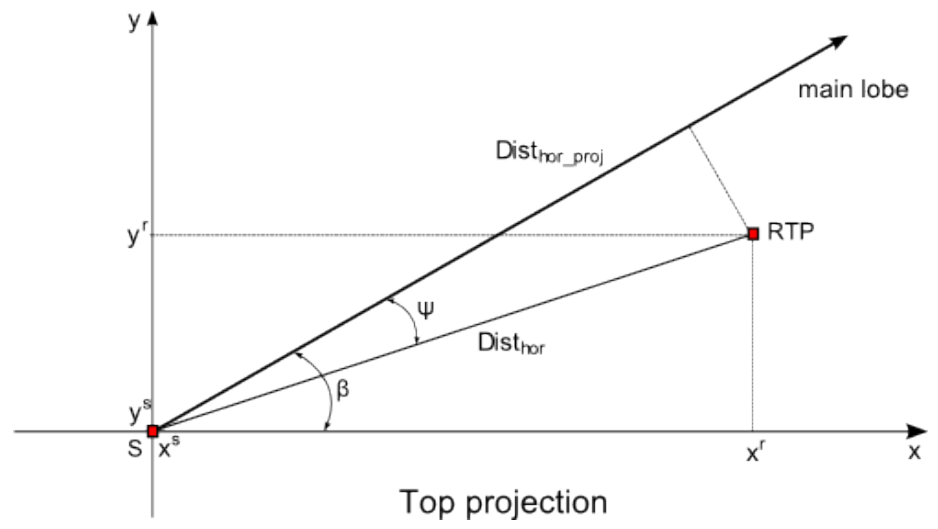

Figure 3.3: Vertical angle calculation - scheme 1

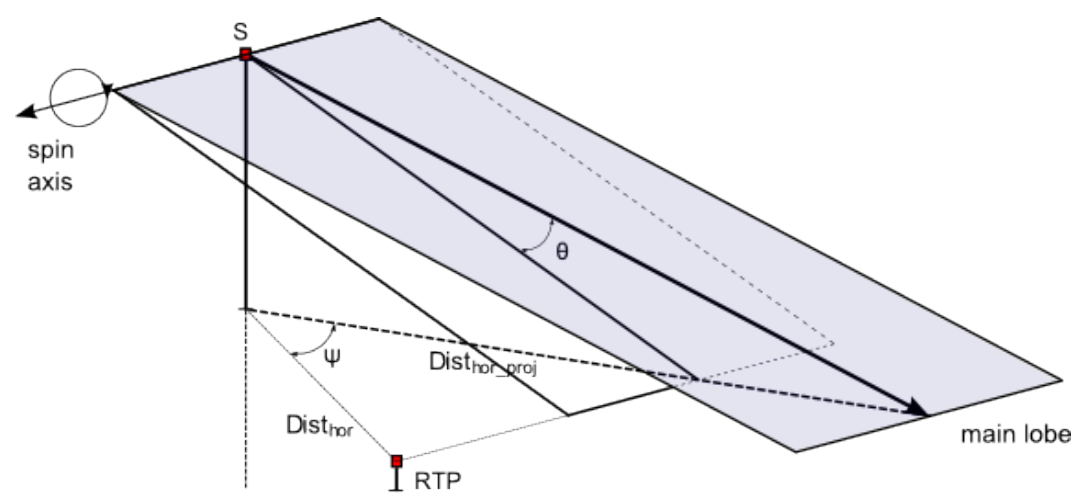

Figure 3.4: Vertical angle calculation - scheme 2 


\begin{tabular}{|c|c|c|c|}
\hline Horizontal angle & Horizontal loss (dBi) & Vertical angle & Vertical loss (dBi) \\
\hline 0 & 0.0 & 0 & 0.0 \\
\hline 1 & 0.0 & 1 & -0.2 \\
\hline 2 & 0.0 & 2 & -0.8 \\
\hline 3 & 0.0 & 3 & -1.8 \\
\hline 4 & -0.1 & 4 & -3.3 \\
\hline 5 & -0.1 & 5 & -5.4 \\
\hline 6 & -0.1 & 6 & -8.3 \\
\hline 7 & -0.2 & 7 & -11.8 \\
\hline 8 & -0.3 & 8 & -15.0 \\
\hline 9 & -0.3 & 9 & -15.0 \\
\hline 10 & -0.4 & 10 & -13.3 \\
\hline 11 & -0.5 & 11 & -12.1 \\
\hline 12 & -0.6 & 12 & -11.9 \\
\hline 13 & -0.7 & 13 & -12.6 \\
\hline 14 & -0.8 & 14 & -14.3 \\
\hline 15 & -0.9 & 15 & -17.3 \\
\hline 16 & -1.0 & 16 & -21.7 \\
\hline 17 & -1.2 & 17 & -24.6 \\
\hline 18 & -1.3 & 18 & -21.0 \\
\hline 19 & -1.5 & 19 & -18.0 \\
\hline 20 & -1.6 & 20 & -16.3 \\
\hline 21 & -1.8 & 21 & -15.8 \\
\hline 22 & -1.9 & 22 & -16.2 \\
\hline 23 & -2.1 & 23 & -17.6 \\
\hline 24 & -2.3 & 24 & -20.1 \\
\hline 25 & -2.4 & 25 & -24.7 \\
\hline 26 & -2.6 & 26 & -36.0 \\
\hline 27 & -2.8 & 27 & -32.0 \\
\hline 28 & -3.0 & 28 & -24.5 \\
\hline 29 & -3.2 & 29 & -21.4 \\
\hline
\end{tabular}

Table 3.1: An example of horizontal and vertical loss table for 60 degrees directional antenna pattern. 
Table 3.2 illustrates the calculation of horizontal, vertical and total losses for various tilt values of antenna. The following parameters have been used for calculation:

$$
\begin{aligned}
& x_{b-s}=400 \\
& y_{b-s}=100 \\
& z_{b-s}=95 \\
& x_{r}=750 \\
& y_{r}=225 \\
& z_{r}=10 \\
& \beta=35 \\
& \text { Dist }_{\text {vert }}=z_{b-s}-z_{r}=85 \\
& \text { Dist }_{\text {hor }}=\sqrt{\left(x_{b-s}-x_{r}\right)^{2}+\left(y_{b-s}-y_{r}\right)^{2}} \approx 371.65 \\
& \psi=\beta-\arctan \left(\frac{y_{b-s}-y_{r}}{x_{b-s}-x_{r}}\right) \approx 15.35 \\
& \text { Dist }_{\text {hor_proj }}=\text { Dist }_{\text {hor }} / \cos (\psi) \approx 385.39 \\
& \theta=\operatorname{round}(12.44-\alpha)
\end{aligned}
$$




\begin{tabular}{|c|c|c|c|c|}
\hline Tilt & $\theta$ & $\begin{array}{c}\text { Vertical loss } \\
(\mathbf{d B i})\end{array}$ & $\begin{array}{c}\text { Horizontal } \\
\text { loss (dBi) }\end{array}$ & $\begin{array}{c}\text { Total loss } \\
(\mathbf{d B i})\end{array}$ \\
\hline 0 & 12 & -11.9 & -0.9 & -12.8 \\
1 & 11 & -12.1 & -0.9 & -13.0 \\
2 & 10 & -13.3 & -0.9 & -14.2 \\
3 & 9 & -15.0 & -0.9 & -15.9 \\
4 & 8 & -15.0 & -0.9 & -15.9 \\
5 & 7 & -11.8 & -0.9 & -12.7 \\
6 & 6 & -8.3 & -0.9 & -9.2 \\
7 & 5 & -5.4 & -0.9 & -6.3 \\
8 & 4 & -3.3 & -0.9 & -4.2 \\
9 & 3 & -1.8 & -0.9 & -2.7 \\
10 & 2 & -0.8 & -0.9 & -1.7 \\
11 & 1 & -0.2 & -0.9 & -1.1 \\
12 & 0 & 0.0 & -0.9 & -0.9 \\
\hline
\end{tabular}

Table 3.2: An example of horizontal and vertical loss calculation for 60 degrees directional antenna pattern.

\subsection{Propagation models for WiMAX at $3.5 \mathrm{GHz}$}

In wireless telecommunication systems, transmission of information between radio entities is performed through electromagnetic waves. During propagation these waves interact with the environment and the signal is attenuated due to the following factors: path distance, terrain pattern, carrier frequency, reflection, diffraction, scattering, freespace loss and absorption by the objects, which causes path loss. Therefore path loss can be defined as the attenuation of the signal strength between transmitter and receiver, measured in $\mathrm{dB}$ : 


$$
Q_{j-k}=B_{j-k}^{P}+B_{j-k}^{G}+R_{i}^{G}-F_{j-k}\left(R_{i}\right)-B_{j-k}^{L_{v e r t}}-B_{j-k}^{L_{h o r}}
$$

where $B_{j-k}^{P}$ and $F_{j-k}\left(R_{i}\right)$ are transmitted and received signal powers, $B_{j-k}^{G}$ and $R_{i}^{G}$ are transmitter and receiver antenna gains respectively, and $B_{j-k}^{L_{v e r t}}$ and $B_{j-k}^{L_{h o r}}$ are combined losses.

Path loss is the main factor characterizing a radio link and serves to predict the effective area of coverage for transmitters and modelling the distribution of signals over different regions. However as each link encounters different attenuating factors and conditions, it is very difficult to formulate the exact losses for different communication systems and the range of frequencies available, in a single mathematical expression. Therefore different models exist for different types of radio links under different conditions.

During preliminary network deployment propagation models play an important role in evaluating the signal characteristics in the network and therefore accuracy of the radio propagation behaviour, and feasibility of potential networks. WiMAX systems can operate in the frequency bands between 2 and $11 \mathrm{GHz}$ and have the benefit of working in NLOS conditions when direct visibility between the communicating antennas is absent. The key feature of WiMAX systems however is to successfully perform under NLOS propagation conditions employing adaptive modulation and coding schemes for different signal to noise ratios. In different propagation conditions with strong interference present or with weak signals due to other phenomena, the system is able to choose the most suitable and robust modulation which ensures transmission.

\subsubsection{Types of propagation models}

There are several types of models to predict path loss, specifically developed for the 3.5 $\mathrm{GHz}$ frequency band covering rural, suburban and urban environments and for different antenna heights [108]. These models can be broadly categorized into the following two main types: 
- Deterministic

- Empirical

Deterministic models became successful for their capability to provide realistic and Site Specific (SISP) radio signal coverage [71]. They are even used to provide wireless channel models, including wideband characteristics such as multiple path delays, and directions of departure and arrival. These characteristics of the channel are essential to evaluate the performance of wideband systems using spatial diversity techniques. The deterministic model makes use of physical laws governing electromagnetic wave propagation. In more detail, the ray-based models [85] use geographical digital models to generate rays (by means of geometrical optics), adding reflected and diffracted contributions to the received signal. As input these models require detailed information on the terrain profile, coordinates and dimensions of buildings and other obstacles. The actual representation of buildings and terrain features can be designed by the use of building databases. The conventional ray-tracing techniques consider only contours of the digital representations of the environment and do not include all the complexity of the small cell environment such as urban furniture and vehicles. However, new propagation models target the lack of representation of the geographical digital modelling, even including irregularities such as diffuse scattering from building walls [48] and lampposts [102].

Empirical models are based on large sets of data/measurements collected for the specific scenario and predict mean path loss as a function of various parameters, e.g. antenna heights, distance, frequency, etc. For any model, the collection of data has to be sufficiently large to provide enough similarity to all kind of situations that can happen in that specific scenario. Empirical models are easier to implement, with less computational cost, but they are less accurate. By definition, an empirical model does not define the exact behaviour of a link, rather, they predict the most likely behaviour the link may exhibit under the specified conditions. It can be split into two subcategories, time dispersive and non-time dispersive [16]. The time dispersive model provides 
us with information about time dispersive characteristics of the channel such as delay spread of the channel during multipath. The Stanford University Interim (SUI) model [56] is the perfect example of this type. Various extensions of Hata [78], COST-231 Hata [45] and ITU-R [16] model are examples of the non-time dispersive empirical model.

\subsubsection{Rapid Pipeline Development Tool}

In this section we present a description of the Rapid Pipeline Development (RPD) Tool, which is used to model the propagation of radio waves at microwave frequencies. This tool has been used to produce propagation information for this thesis. It has been developed by the Radio Communications Research Unit (RCRU) which is part of STFC Rutherford Appleton Laboratory and used widely across the industry and academic projects [2, 62].

RPD is a site-specific tool and based on ray-tracing techniques to construct link profiles and to predict radio wave propagation in a dense urban environment. It takes as input a 3-D model of the proposed network area, ie the position of transmitters and detailed city datasets, comprising the outlines of buildings, terrain and vegetation features, and outputs path loss values at each reception test points from each candidate base station.

The main focus of research at RCRU has been on developing algorithms to calculate propagation characteristics over terrain or buildings for RPD. These algorithms make use of extensions to ITU-R P. $452^{1}$, mesh path loss calculation and bottleneck evaluation, and a full ray launching algorithm modelling reflection, transmission, diffraction (shadow region only) and scatter.

RPD relies on the use of the freely available Visualisation Toolkit (VTK) library [4] to manipulate and process 3D data. A simple graphical user interface (GUI) allows

\footnotetext{
${ }^{1}$ Prediction procedure for the evaluation of interference between stations on the surface of the Earth at frequencies above about $0.1 \mathrm{GHz}$, International Telecommunication Union Recommendation.
} 
modules to be connected together with data pipelines. The following classes have been formed to support radio propagation research:

- Transmitter

- Antenna

- Receiver Test Points

- Channel Impulse Responses

- Surface Properties

The functional unit of the VTK is the visualisation pipeline which represents data in the form of objects: process objects (objects to operate on data) and arrow connections between objects (an indicated direction data flow).

RPD allows to create, save and retrieve pipelines visualising $3 \mathrm{D}$ urban data. It can be saved in the form of an RPD application. An example of the visualisation pipeline application is depicted in Figure 3.5 .

\section{Architecture of RPD}

The network data can be loaded to RPD in various formats. The main data format however is a DXF file - Data Exchange Format/Data Interchange Format which can be purchased from the National Remote Sensing Centre. This file includes 3D information including the positions of the corners of the buildings, as well as terrain and vegetation - bushes, trees. Once it is loaded the 3D data may be checked for spurious points, decimated, triangulated, quantised, cut, etc. using the filters provided in the RPD.

After all the required data is imported the coverage calculations can be done using the following filters:

- Coverage Profiles LOS (Line-Of-Sight) 


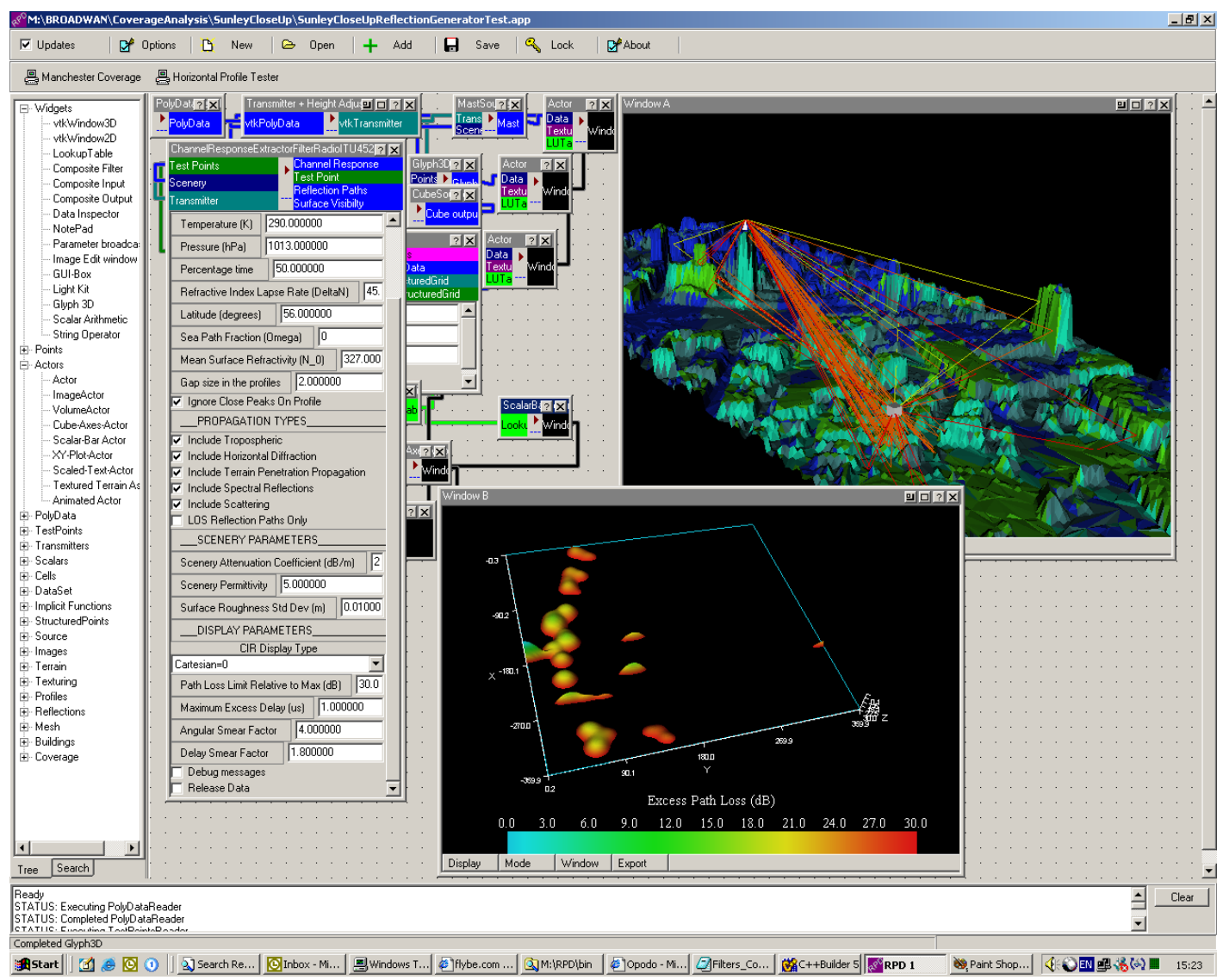

Figure 3.5: RDP application

- Coverage Profiles ITU-R 452 [86, 87]

- Coverage Profiles COST210 [1]

All three filters consider the straight-line profile between the transmitter and the receiver (TestPoint) and the heights along that profile.

The filters use three inputs: 


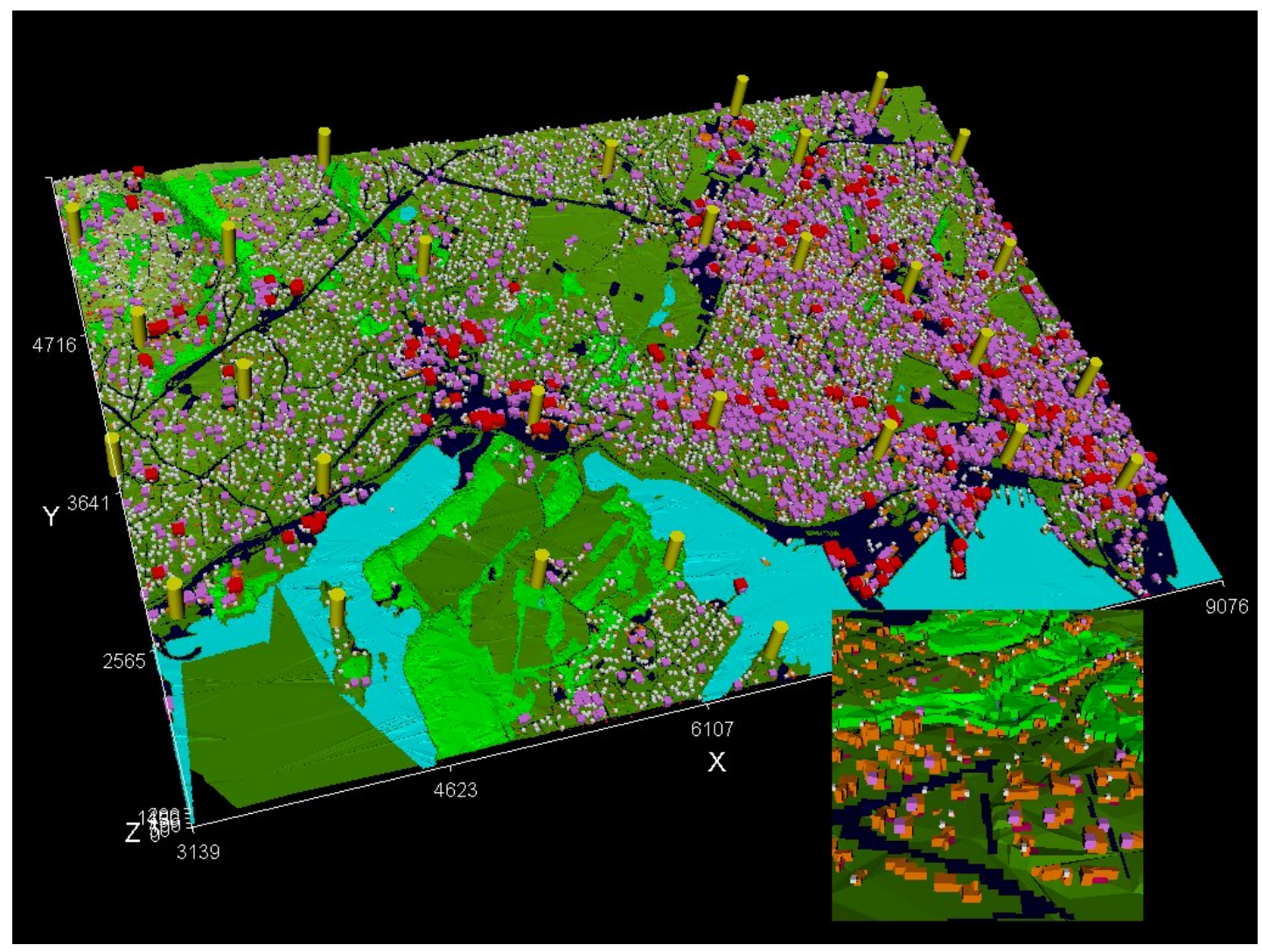

Figure 3.6: 3D visualisation of an example network terrain

1. Scenery - buildings, structures and vegetation inside the town.

2. TestPoints - the points where the EM field is examined.

3. Transmitters - defined as a single point with a number of attributes that can be set by the user.

An example of the visual representation of the 3D network model can be seen on Figure 3.6 


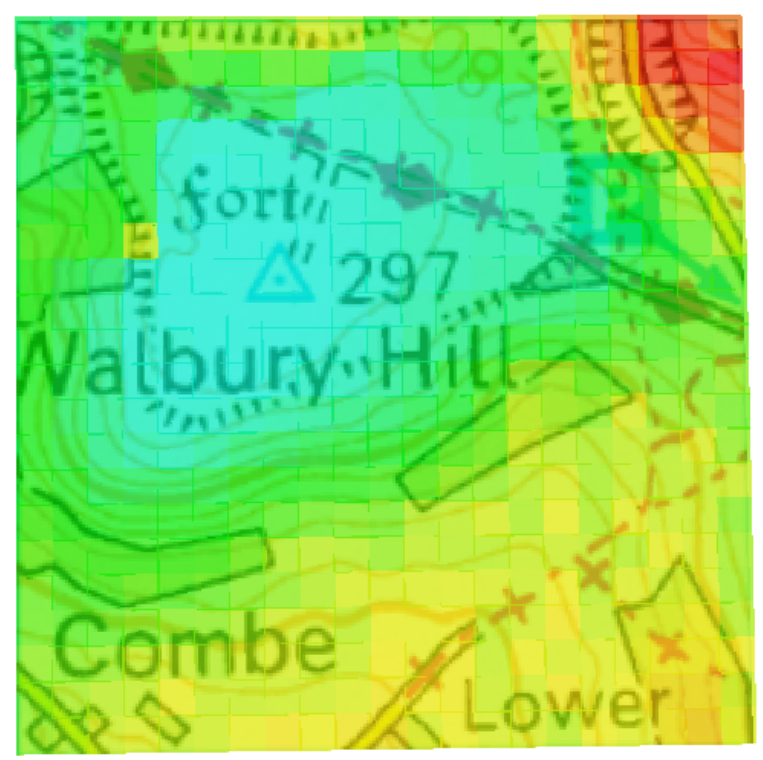

Path Loss from Chilbolton at $1.2 \mathrm{GHz}(\mathrm{dB})$ (at $10 \mathrm{~m}$ above ground height)

$\begin{array}{lllllllll}120.0 & 130.0 & 140.0 & 150.0 & 160.0 & 170.0 & 180.0 & 190.0 & 200.0\end{array}$

Figure 3.7: Path losses base station and test points calculated using ITU-R P.452 module in RPD.

The output of the tool (coverage filters) is produced in the form of a file which contains the array of loss values (in decibels) for all base station/receiver pairs. An example of the antenna coverage with accounted path loss grading can be seen in Figure 3.7 . 


\subsection{Coverage}

After the BS is mounted and configured (e.g. power, azimuth and tilt set) a coverage area can be identified. An RTP is considered to be covered by a sector if the received signal strength is strong enough, i.e. above the sensitivity threshold. The sensitivity level $R_{i}^{R x}$ defined at the RTP determines the minimum received power level required for the user equipment to identify the signal with an acceptable level of errors among the noise and interference. The receiver must be able to decode data bits with a Bit Error Rate $(\mathbb{B E R})$ less than $1 \times 10^{-6}$. This may vary with different equipment and user service requirements, as the threshold defines the lowest transmission data rate.

The downlink received signal strength from sector $B_{j-k}$ at site $S_{k}$ to RTP $R_{i}$ can be calculated as follows:

$$
\begin{aligned}
F_{j-k}\left(R_{i}\right)= & B_{j-k}^{P}+B_{j-k}^{G}+R_{i}^{G}-Q_{k-i} \\
& -B_{j-k}^{D_{\text {hor }}}\left(B_{j-k}^{\beta}\right)-B_{j-k}^{D_{\text {vert }}}\left(B_{j-k}^{\alpha}\right)
\end{aligned}
$$

where $B_{j-k}^{P}$ is the emitted power of the BS sector $B_{j-k}$ at the site $S_{k}, B_{j-k}^{G}$ is the BS's antenna gain, $R_{i}^{G}$ is the user's antenna gain, $Q_{k-i}$ is the path loss between site $S_{k}$ and reception point $R_{i}, B_{j-k}^{D_{h o r}}$ and $B_{j-k}^{D_{v e r t}}$ are the horizontal and vertical losses for the type of antenna $B_{j-k}^{A}$. Finally $B_{j-k}^{\beta}$ and $B_{j-k}^{\alpha}$ are the angles between the main radiation beam axis and $R_{i}$ in the horizontal and vertical planes respectively.

In order to be covered a user must satisfy the following condition:

$$
F_{j-k}\left(R_{i}\right) \geq R_{i}^{R x}
$$

The receiver sensitivity is a function of modulation and coding type, Signal to Noise Ratio (SNR) and channel size. All the specified conditions are defined in [6] and presented in Table 3.3 . 


\begin{tabular}{|l|c|c|c|c|c|c|c|}
\hline \multirow{2}{*}{ Bandwidth } & \multicolumn{6}{|c|}{ Modulation and coding } \\
\cline { 2 - 8 } & BPSK & \multicolumn{2}{|c|}{ QPSK } & \multicolumn{2}{c|}{ 16 QAM } & \multicolumn{2}{c|}{64 QAM } \\
\cline { 2 - 8 } & $1 / 2$ & $1 / 2$ & $3 / 4$ & $1 / 2$ & $3 / 4$ & $2 / 3$ & $3 / 4$ \\
\hline $1.75 \mathrm{MHz}$ & -93.7 & -90.7 & -88.9 & -83.7 & -81.9 & -77.4 & -75.7 \\
\hline $3.5 \mathrm{MHz}$ & -90.7 & -87.7 & -85.9 & -80.7 & -78.9 & -74.4 & -72.7 \\
\hline $7.0 \mathrm{MHz}$ & -87.6 & -84.6 & -82.8 & -77.6 & -75.8 & -71.3 & -69.6 \\
\hline $10.0 \mathrm{MHz}$ & -86.1 & -83.1 & -81.3 & -76.1 & -74.3 & -69.8 & -68.1 \\
\hline $20.0 \mathrm{MHz}$ & -83.0 & -80.0 & -78.2 & -73.0 & -71.2 & -66.7 & -65.0 \\
\hline
\end{tabular}

Table 3.3: Receiver sensitivity (dBm)

In addition the following set of variables is introduced to explicitly define covered RTPs:

$$
\begin{aligned}
R_{i}^{c o v} & =\left\{\begin{array}{l}
1-\text { if } R T P \text { is covered } \\
0-\text { otherwise }
\end{array}\right. \\
R_{i-j-k}^{c o v} & =\left\{\begin{array}{l}
1-\text { if } R T P \text { is covered by sector }(j) \text { at site }(k) \\
0-\text { otherwise }
\end{array}\right.
\end{aligned}
$$

Generally in 3G mobile networks a typical coverage level desired by operators is more than $95 \%$ of the area.

\subsection{Service and Traffic}

In this thesis we consider the peak busy hour (PBH) traffic demand snapshot for capacity planning. In telecommunications systems, the $\mathrm{PBH}$ is a period during which occurs the maximum total traffic load in a given 24-hour period. The PBH activity level is a challenging task for an operator to predict with any degree of accuracy. It depends on applications, customer mix, etc. The process is further complicated by the 
fact that there will not be any prior history for many of the new services to be offered on which operator can base traffic estimates. Where there does seem to be consensus however, is that traffic will be increasingly more data-centric. With data-centric traffic, downlink traffic is expected to dominate. For that reason we will focus exclusively on DL projections for capacity planning purposes.

When the user is covered by base station sector by receiving an adequate signal strength, in order to get service the following conditions must be met:

1. serving BS must be the best server providing the strongest signal

$$
F_{j-k}\left(R_{i}\right)=\max \left\{F_{b-s}\left(R_{i}\right): 1 \leq\left|B_{b-s}\right| \leq N_{R_{i}}^{c o v}\right\}
$$

where $N_{R}^{\text {cov }}$ is the number of stations covering RTP $R$

$$
N_{R_{i}}^{c o v}=\sum_{k \in S, j \in B_{S_{k}}} R_{j-k}^{c o v}
$$

2. the SINR level at the RTP is greater than the given service threshold

3. the traffic demand of the user is fully satisfied

4. the additional traffic of the user is not overloading the overall capacity of the BS

Similar to notations for covered users we introduce the following variables:

$$
\begin{aligned}
R_{i}^{\text {serv }} & =\left\{\begin{array}{l}
1-\text { if } R T P(i) \text { is serviced } \\
0-\text { otherwise }
\end{array}\right. \\
R_{i-j-k}^{\text {serv }} & =\left\{\begin{array}{l}
1-\text { if } R T P(i) \text { is serviced by sector }(j) \text { at site }(k) \\
0-\text { otherwise }
\end{array}\right.
\end{aligned}
$$




\subsection{Transmit power and subchannelization}

There are several techniques employed in WiMAX to achieve long range coverage including high transmission power, adaptive modulation and coding schemes, and subchannelization.

\subsubsection{WiMAX transmission power}

Although high transmission power level is crucial for long distance communications, it must be balanced with high efficiency of the system in order to ensure robust links, high transmission rates and a wide range for WiMAX services.

WiMAX base station is limited to approximately $+45 \mathrm{dBm}$, as compared to a WiMAX mobile station (MS) which transmits at a maximum level of $+23 \mathrm{dBm}(200 \mathrm{~mW})$. These limitations vary for different countries, regulatory bodies, technological limits and usage models. During normal operation, a base station will normally transmit sufficient power for the system to function properly. Excessive output power may cause unnecessary interference in the network.

As opposed to $3 \mathrm{G}$ cellular systems, WiMAX operating at higher modulation orders requires a much better SNR level in order to maximize throughput. Moreover, as in cellular networks, WiMAX is severely uplink limited, meaning that a user station can receive a signal from a $\mathrm{BS}$ easier than for the $\mathrm{BS}$ to hear a users relatively low power transmission. This can be seen from the marginal difference of $20 \mathrm{~dB}$ between downlink and uplink power levels - from/to BS to/from user respectively.

However, WiMAX employing OFDMA communication with a frequency subchannelization technique allows a user to occupy only a subset of all the available subchannels. This in turn allows concentration of the user transmit power over a smaller range of frequencies. The net signal gain is $10 * \log \left(N_{\text {total }} / N_{\text {used }}\right)$ which is achieved relative to a 
BS transmitting on all subcarriers. $N_{\text {used }}$ is the number of subcarriers assigned to the user, and $N_{\text {total }}$ is the total number of subcarriers available.

Another technique to compensate the link imbalance is the usage of lower order modulation for the user station. This allows the user to communicate with the BS using less transmit power but sacrificing the uplink throughput, since fewer bits are transmitted per subcarrier with lower order modulation.

\subsubsection{Subchannelization}

The ODFMA frame consists of symbols in the time domain and subcarriers in the frequency domain. In order to create a minimal transmission unit in an OFDMA symbol, the modulated symbols are mapped on to subcarriers [104].

Definition 3.5.1. Subchannel. As defined in the IEEE 802.16e-2005 standard, is a logical collection of subcarriers.

Definition 3.5.2. Permutation base. $B_{j-k}^{\text {PermBase }}$ is an integer ranging from 0 to 31 , which identifies the particular BS and is specified by the MAC layer.

Definition 3.5.3. Physical Cluster. A cluster is composed of 14 adjacent subcarriers, including 12 data and 2 pilot. There are 120 physical clusters in total for a 2048-FFT, which are contiguous in the frequency domain, (Figure 3.10).

Definition 3.5.4. Logical Cluster. A renumbering sequence is applied to physical clusters to form logical clusters. It is defined in [11]. These clusters are not contiguous in the frequency domain.

Definition 3.5.5. Group. This consists of a set of logical clusters. There are six groups in total, divided into odd and even numbered groups, with odd numbered groups containing 24 clusters, whereas even numbered groups contain 16 logical clusters for the 2048-FFT. The subcarriers within each group are indexed separately starting from 'O'. 
Therefore, even and odd groups will have subcarriers indexed 0-335 and 0-225 respectively. Thereafter, pilot subcarriers are identified and remainder of the subcarriers are re-indexed to 0-287 and 0-194 for even and odd groups respectively. Subchannels in the amount of '12' and '8' are formed from even and odd groups respectively using inner permutation.

Definition 3.5.6. Inner Permutation. This stage involves the formation of subchannels from the subcarrier of logical clusters of a group.

Definition 3.5.7. Outer Permutation. The transformation of physical clusters into logical clusters by means of renumbering is called Outer Permutation.

Figure 3.8 illustrates the pattern allocation of subchannels for an OFDMA frame where several mobile stations may transmit in the same time slot over several subchannels. Subcarriers forming the subchannel need not be adjacent.

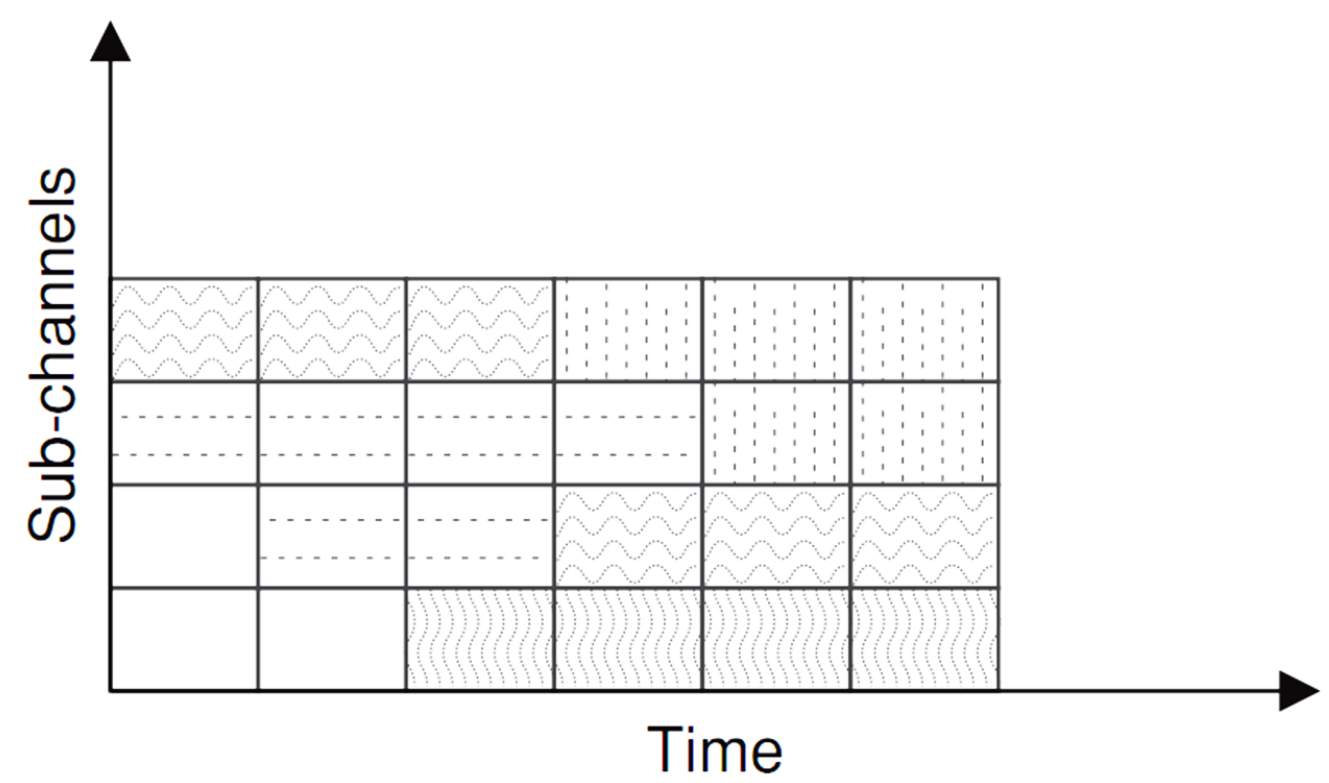

Figure 3.8: OFDMA

Allocating subcarriers to subchannels is a difficult and ongoing research problem which must consider issues such as mobility, Adaptive Antenna Steering (AAS) support and different optimization criteria. The number of subcarriers and their distribution within 
the subchannel depend on the subcarrier permutation scheme. The IEEE 802.16e2005 standard provides examples of several permutations [148]. There are two types of subcarrier permutation mode:

- diversity (distributed) - subcarriers are distributed throughout the frequency band.

- contiguous - subcarriers are adjacent to each other.

A distributed subcarrier permutation scheme gives better frequency diversity and intercell interference averaging, where subcarriers are distributed pseudo-randomly to form a subchannel. It minimizes the probability of using the same subcarriers in adjacent cells. This family includes DL Fully Used Subcarriers (DL FUSC), DL Partially Used Subcarriers (DL PUSC), UL PUSC, and optional permutations such as Optional DL Distributed Subcarrier Permutation: Fully Used Subchannelization (OFUSC), Optional UL Distributed Subcarrier Permutation: Partially Used Subchannelization (OPUSC) and Optional DL and UL Adjacent Subcarrier Permutation: Advanced Modulation and Coding (AMC).

While, a contiguous subcarrier permutation is more desirable for beamforming and allows the system to exploit multi-user diversity by choosing the subchannel with the best frequency response. Subchannels in this mode are formed from a group of adjacent subcarriers.

The trade-off between mobility and throughput is achieved through the use of these permutation schemes. Adjacent schemes are best for fixed, portable, or low mobility environments whereas diversity modes perform well in mobile applications.

Since the aim of this thesis is to plan Mobile WiMAX networks, the permutation scheme used is DL PUSC.

Definition 3.5.8. A slot is a minimum possible data allocation unit in the IEEE 802.16 standard. 


\begin{tabular}{|l|l|}
\hline Permutation scheme and commu- & Slot definition \\
\hline nication mode & \\
\hline Downlink FUSC, downlink OFUSC & 1 subchannel x 1 OFDMA symbol \\
Downlink PUSC & 1 subchannel x 2 OFDMA symbols \\
Uplink PUSC, uplink additional & 1 subchannel x 3 OFDMA symbols \\
PUSC & \\
Uplink and downlink AMC & 1 subchannel x (1,2 or 3) OFDMA symbols \\
\hline
\end{tabular}

Table 3.4: Slot structure

\begin{tabular}{|l|c|c|c|c|}
\hline PARAMETERS & \multicolumn{4}{|c|}{ VALUES } \\
\hline System bandwidth (MHz) & 1.25 & 5 & 10 & 20 \\
FFT size (NFFT) & 128 & 512 & 1024 & 2048 \\
Used subcarriers & 85 & 421 & 841 & 1681 \\
Data subcarriers & 72 & 360 & 720 & 1440 \\
Pilot subcarriers & 12 & 60 & 92 & 184 \\
Left-guard subcarriers & 22 & 46 & 92 & 184 \\
Right-guard subcarriers & 21 & 45 & 91 & 183 \\
Clusters/subchannels & $6 / 3$ & $30 / 15$ & $60 / 30$ & $120 / 60$ \\
\hline
\end{tabular}

Table 3.5: Parameters of DL distributed subcarrier permutation (PUSC)

The exact definition of a slot depends on the number of factors - uplink or downlink subframe, PUSC or FUSC permutation. These variations are reflected in Table 3.4 .

\subsubsection{PUSC structure}

\section{Dividing subcarriers into clusters - Physical Clusters}

First, used subcarriers which include data and pilot are divided into clusters. The partitioning of the channel into different types of subcarriers for the most common channel sizes is provided in Table 3.5 . 
Each cluster comprises of two OFDM symbols in the time domain and fourteen adjacent subcarriers in the frequency domain, with 4 pilot and 24 data subcarriers in each cluster in total. Then clusters are renumbered with a pseudo-random numbering sequence to redistribute the logical identities of clusters.

The distribution of subcarriers to subchannels in DL PUSC is illustrated in Figure 3.9.

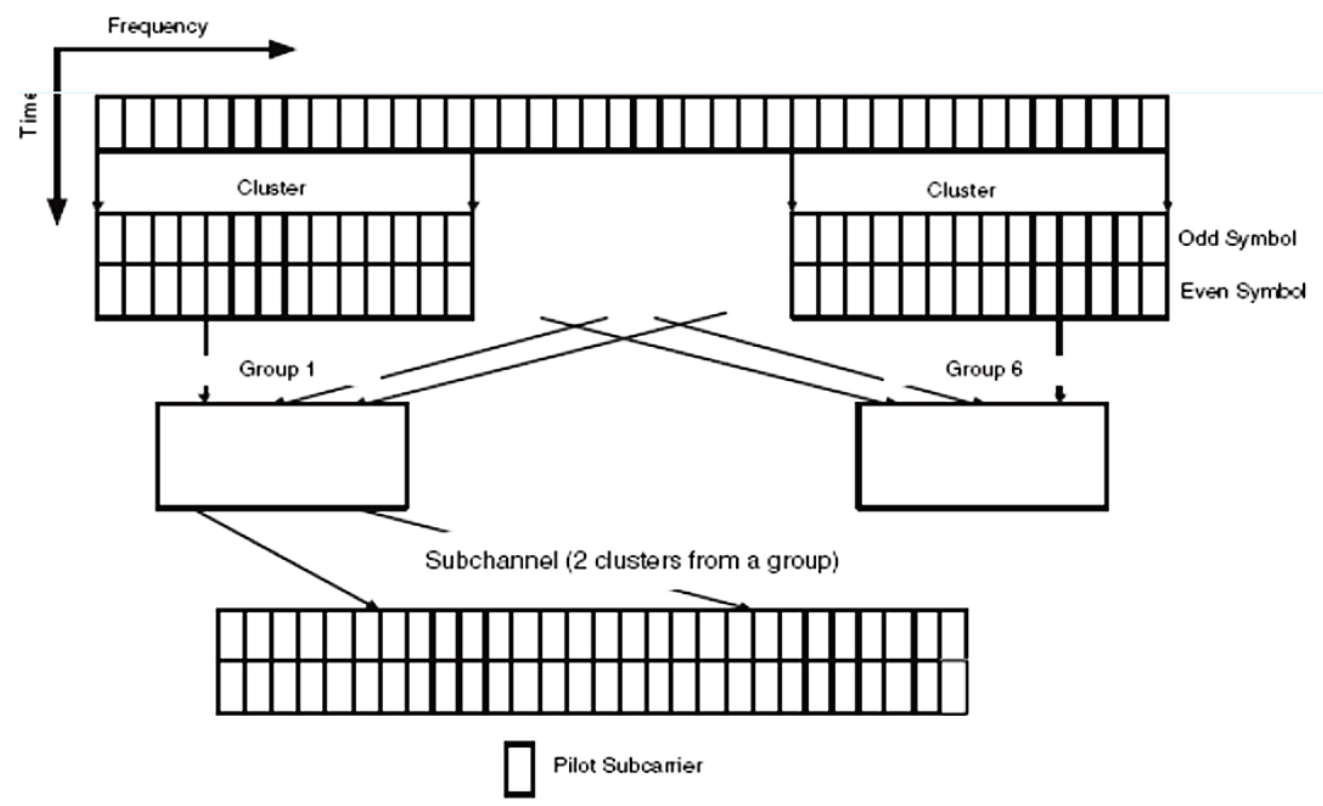

Figure 3.9: DL PUSC permutation sequence [from [104]]

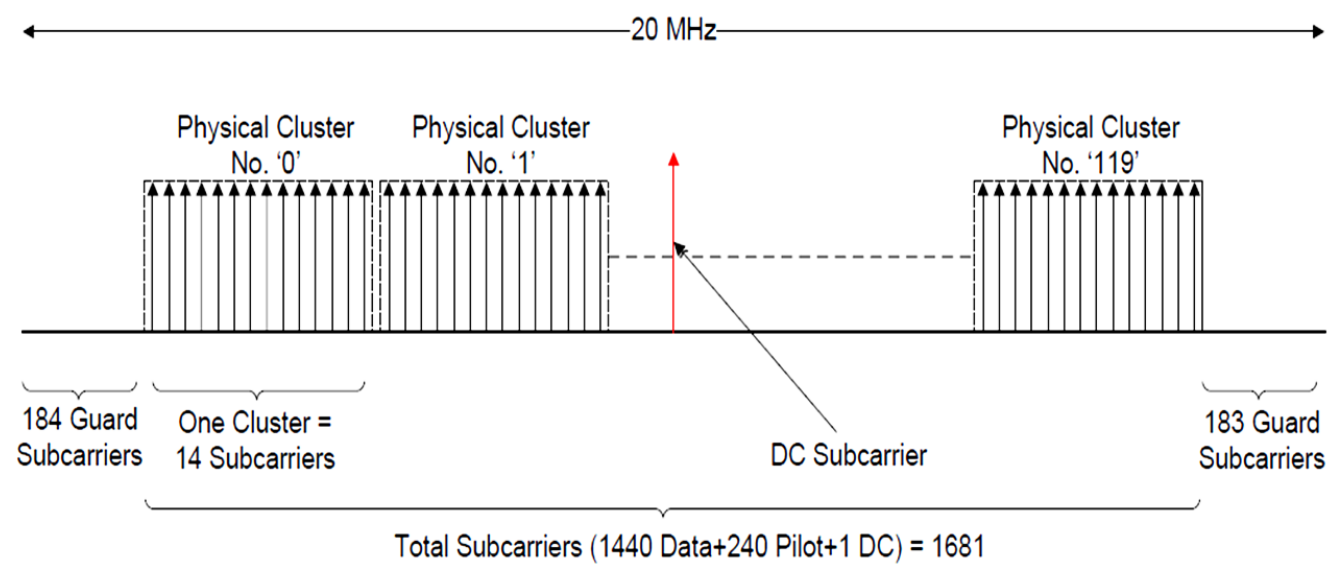

Figure 3.10: DL PUSC physical clustering [from [104]]

As an example, a 20MHz channel with an overall 2048 subcarriers is observed. After removing guard carriers this leaves 1440 of used subcarriers which are divided into 
120 clusters. Initially clusters are numbered with their Physical sequence between 0 and 119, (Figure 3.10).

\section{Renumbering - Logical Clusters}

These clusters are then renumbered into Logical Numbers $(\mathrm{LN})$ with the use of $B_{j-k}^{\text {PermBase }}$ parameter.

Specifically the physical clusters obtained in step 1 are renumbered to form Logical clusters (LC) using equation 3.2. This stage is called the Outer Permutation.

$$
L C=R S(P C)+13 B_{j-k}^{\text {PermBase }}
$$

The $R S$ is a renumbering sequence which is given in [11]:

$$
\left\{\begin{array}{cccccccccc}
6 & 108 & 37 & 81 & 31 & 100 & 42 & 116 & 32 & 107 \\
30 & 93 & 54 & 78 & 10 & 75 & 50 & 111 & 58 & 106 \\
23 & 105 & 16 & 117 & 39 & 95 & 7 & 115 & 25 & 119 \\
53 & 71 & 22 & 98 & 28 & 79 & 17 & 63 & 27 & 72 \\
29 & 86 & 5 & 101 & 49 & 104 & 9 & 68 & 1 & 73 \\
36 & 74 & 43 & 62 & 20 & 84 & 52 & 64 & 34 & 60 \\
66 & 48 & 97 & 21 & 91 & 40 & 102 & 56 & 92 & 47 \\
90 & 33 & 114 & 18 & 70 & 15 & 110 & 51 & 118 & 46 \\
83 & 45 & 76 & 57 & 99 & 35 & 67 & 55 & 85 & 59 \\
113 & 11 & 82 & 38 & 88 & 19 & 77 & 3 & 87 & 12 \\
89 & 26 & 65 & 41 & 109 & 44 & 69 & 8 & 61 & 13 \\
96 & 14 & 103 & 2 & 80 & 24 & 112 & 4 & 94 & 0
\end{array}\right\}
$$

\section{Grouping clusters}

Logical clusters are grouped to form 6 major groups and numbered from 0 to 5 . For a 2048-FFT, the even groups (0, 2 and 4) include 24 logical clusters each, while odd 
groups (1, 3 and 5) have 16 logical clusters. The formation of the groups is shown in Figure 3.11. In order to appreciate how this outer permutation gives frequency diversity, Figure 3.12 shows the distribution of logical clusters of group '0' with respect to 120 physical cluster positions.

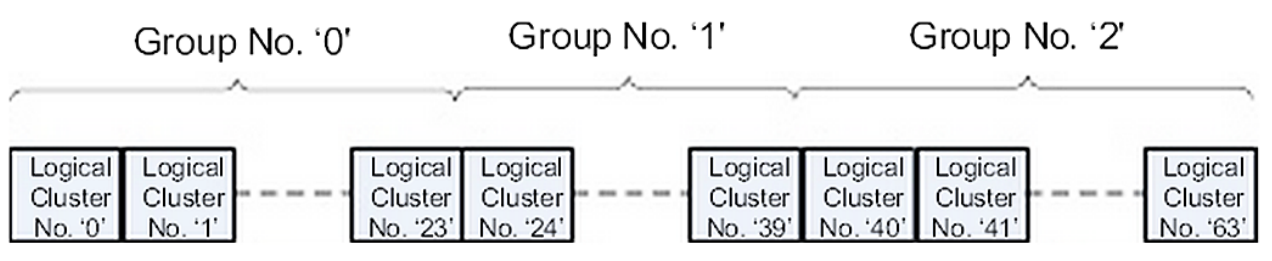

\begin{tabular}{|c|c|c|c|c|c|c|c|c|c|}
\hline $\begin{array}{l}\text { Logical } \\
\text { Cluster } \\
\text { No. ' } 64 \text { ' }\end{array}$ & --- & $\begin{array}{l}\text { Logical } \\
\text { Cluster } \\
\text { No. } 79\end{array}$ & $\begin{array}{l}\text { Logical } \\
\text { Cluster } \\
\text { No. ' } 80 \text { ' }\end{array}$ & $\begin{array}{l}\text { Logical } \\
\text { Cluster } \\
\mathrm{No} \cdot 81 \text { ' }\end{array}$ & --- & $\begin{array}{l}\text { Logical } \\
\text { Cluster } \\
\text { No.'103' }\end{array}$ & $\begin{array}{l}\text { Logical } \\
\text { Cluster } \\
\text { No.'104' }\end{array}$ & $---\cdots$ & $\begin{array}{l}\text { Logical } \\
\text { Cluster } \\
\text { No.'103' }\end{array}$ \\
\hline
\end{tabular}

Group No. ' 3 ' $\underbrace{}_{\text {Group No. ' } 4 \text { ' }} \overbrace{\text { Group No. ' } 5 \text { ' }}$

Even Numbered Groups = 24 Clusters
Odd Numbered Groups = 16 Clusters

Figure 3.11: DL PUSC grouping clusters [from [104]]

\section{Pilot subcarriers}

The positions of the pilot subcarriers are different for an odd and even OFDM symbol.

Within the 14 subcarriers numbered as $0-13$, for odd symbols the pilots are marked as '4' and '8', for even '0' and '12', (Figure 3.13). 


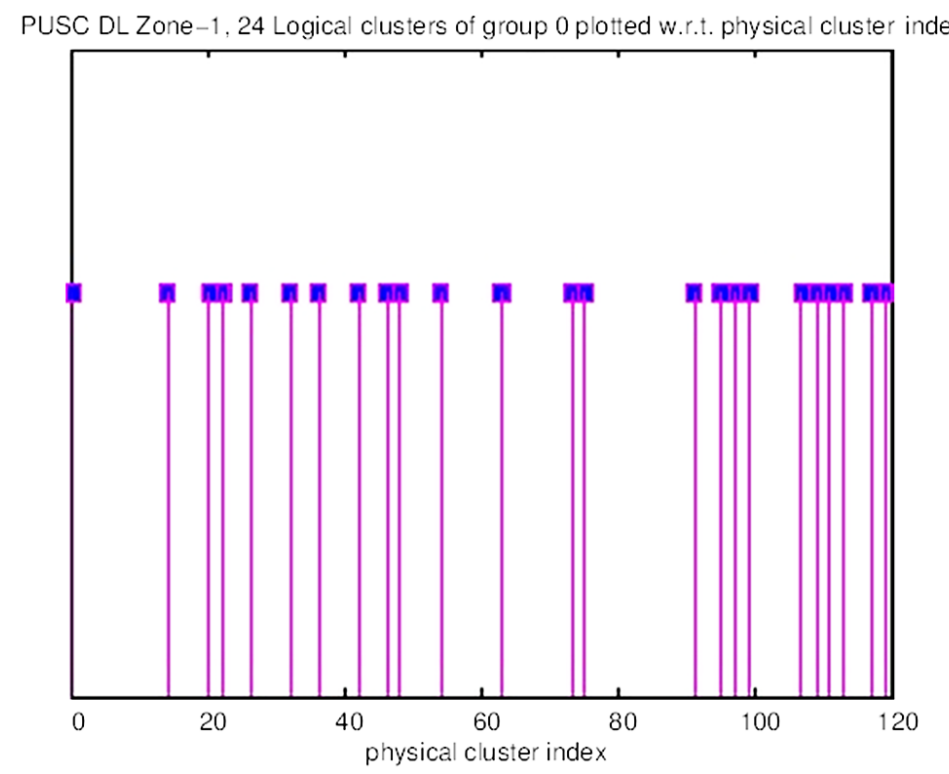

Figure 3.12: DL PUSC distribution of subcarriers [from [104]]

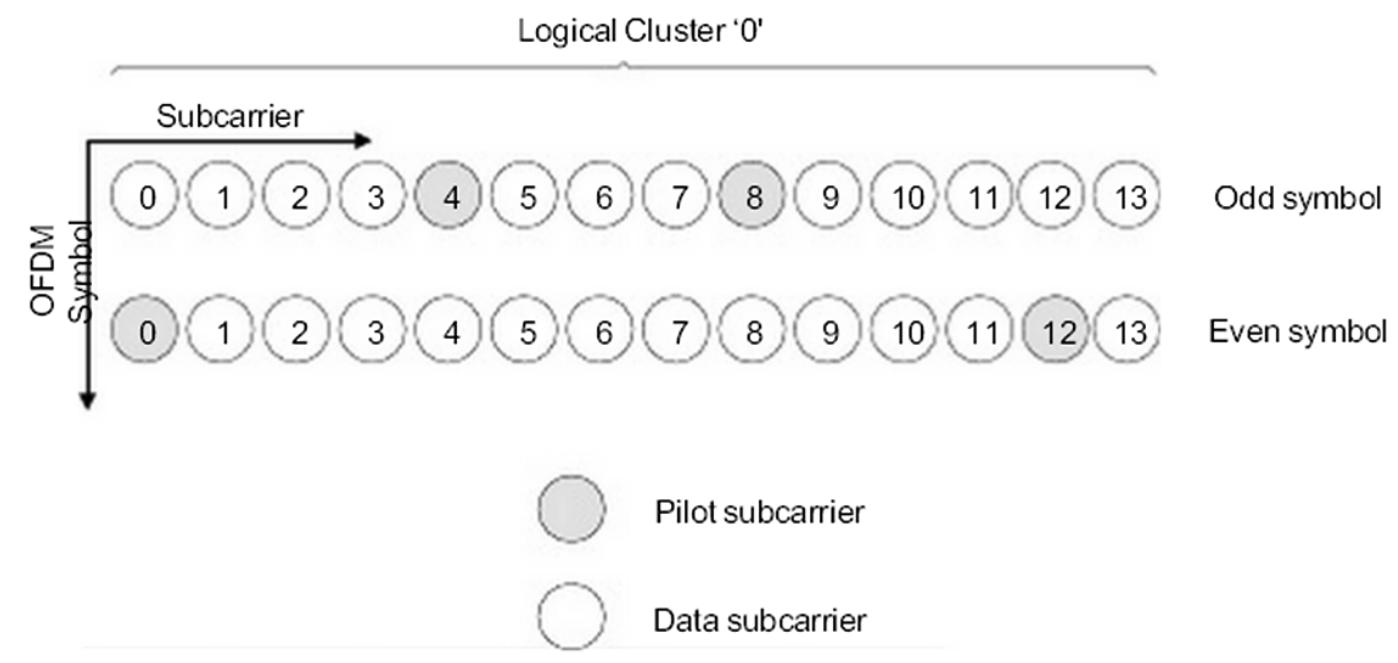

Figure 3.13: DL PUSC - positioning of pilot subcarriers [from [104]]

\section{Forming subchannels}

Finally, subcarriers of a group are distributed to subchannels using the inner permutation given in Eq.111 of [13]. This formula gives the subcarrier index within the group: 


$$
\begin{aligned}
& \operatorname{subcarrier}(k, s)=N_{s c r} n_{k}+P_{S}\left[n_{k} \bmod N_{s c h}\right]+B_{i-j}^{\text {PermBase }} \bmod N_{s c h} \\
& \text { where } \\
& \text { subcarrier }(k, s) \quad \text { is the index of subcarrier } k \text { within a group (i.e., with 2048-FFT, } \\
& \text { in group ' } 0 \text { ', it ranges from (0-287) } \\
& \text { S } \\
& \text { is the index number of a subchannel in a group, from the set } \\
& \text { [0... } \left.N_{s c h-1}\right] \text { (i.e., with 2048-FFT, } N_{\text {sch }} \text { for even and odd groups } \\
& \text { are } 12 \text { and } 8 \text { respectively) } \\
& n_{k} \quad=(k+13 s) \bmod N_{s c r} \\
& k \quad \text { is the subcarrier-in-subchannel index from the set } \\
& {\left[0 \ldots N_{s c r}-1\right]} \\
& N_{s c r} \quad \text { is always equal to } 24 \text { for PUSCDL } \\
& P_{S}[.] \quad \text { is the series obtained by rotating basic permutation } \\
& \text { sequence cyclically to the left } s \text { times (Table-310 [3]). } \\
& P_{S}[.]=\{6,9,4,8,10,11,5,2,7,3,1,0\} \text { and } \\
& \{7,4,0,2,1,5,3,6\} \text { for even and odd groups respectively. }
\end{aligned}
$$

The effect of the inner permutation on frequency diversity with the subcarriers of subchannel ' 0 ' of group ' 0 ' is depicted on Figure 3.14. The effect of both the permutations (inner and outer) can be seen on Figure 3.15.

\subsubsection{Slot assignment formulation}

As defined in the previous section the minimum time/frequency resource allocation unit which can be assigned to a single user for data transmission is a slot. The full set of slots in the downlink subframe is denoted as $L$. According to the selected permutation scheme the slot is formed of 1 subchannel x (1, 2 or 3) OFDMA symbols, (Table 3.4). At each super-frame all the base stations are requesting Channel State Information (CSI) from their subscribers. The CSI represents the interference state and therefore determines the SINR levels of all the channels. According to the SINR the BS selects the most efficient modulation and coding scheme for the user to maximize 
PUSC DL Zone $=1$, group $=0$, PermBase $=0$ and symbol $=$ Odd 24 Data subcarriers of subchannel 0 plotted $w$.r.t. group subcarrier index

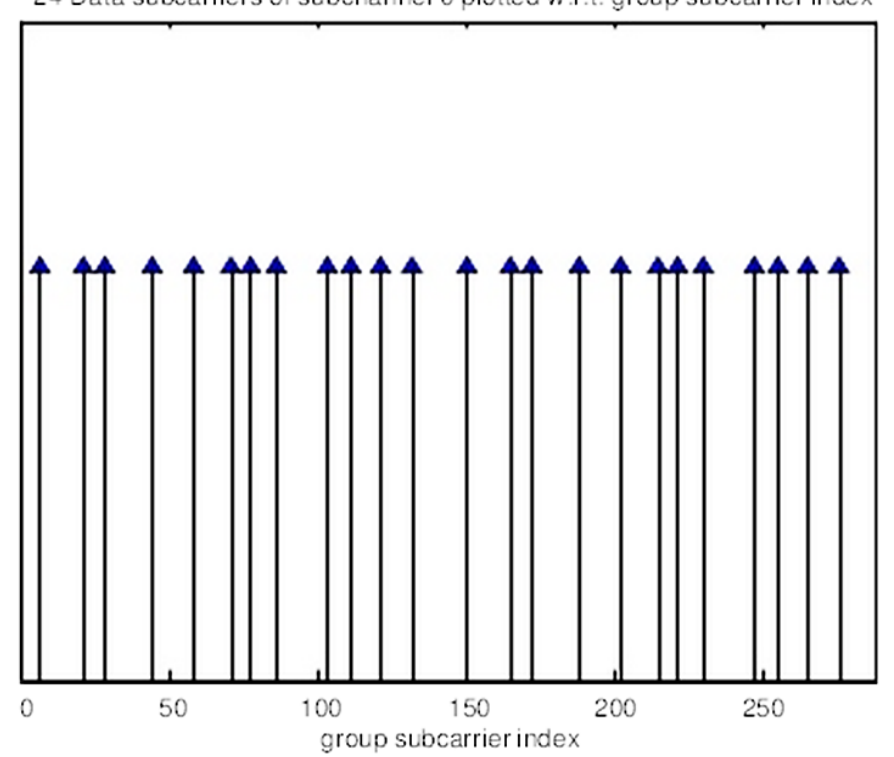

Figure 3.14: Subcarriers of subchannel plotted against group subcarrier index [from [104]].

its achievable bit-rate.

All available slots are shared among the users within a single base station. The slot allocation problem is defined as a non-linear integer programming where for each user a slot allocation matrix is given $\Delta_{R_{j}}$ and denoted as:

$$
\begin{aligned}
\delta_{j}^{i}= & \left\{\begin{array}{l}
1-\text { if slot }\left(L_{i}\right) \text { is assigned to the user at } R T P\left(R_{j}\right) \\
0-\text { otherwise }
\end{array}\right. \\
& L_{i} \in L, R_{j} \in R
\end{aligned}
$$

If a slot is used by a cell it is denoted as:

$$
\begin{aligned}
\delta_{j-k}^{i}= & \left\{\begin{array}{l}
1-\text { if slot }\left(L_{i}\right) \text { is used at sector }\left(B_{j-k}\right) \text { of site }\left(S_{k}\right) \\
0-\text { otherwise }
\end{array}\right. \\
& L_{i} \in L, B_{j} \in B_{S_{k}}, S_{k} \in S
\end{aligned}
$$

We make the following assumptions in this thesis: 
PUSC DL Zone $=1$, group $=0$, PermBase $=0$ and symbol=Odd

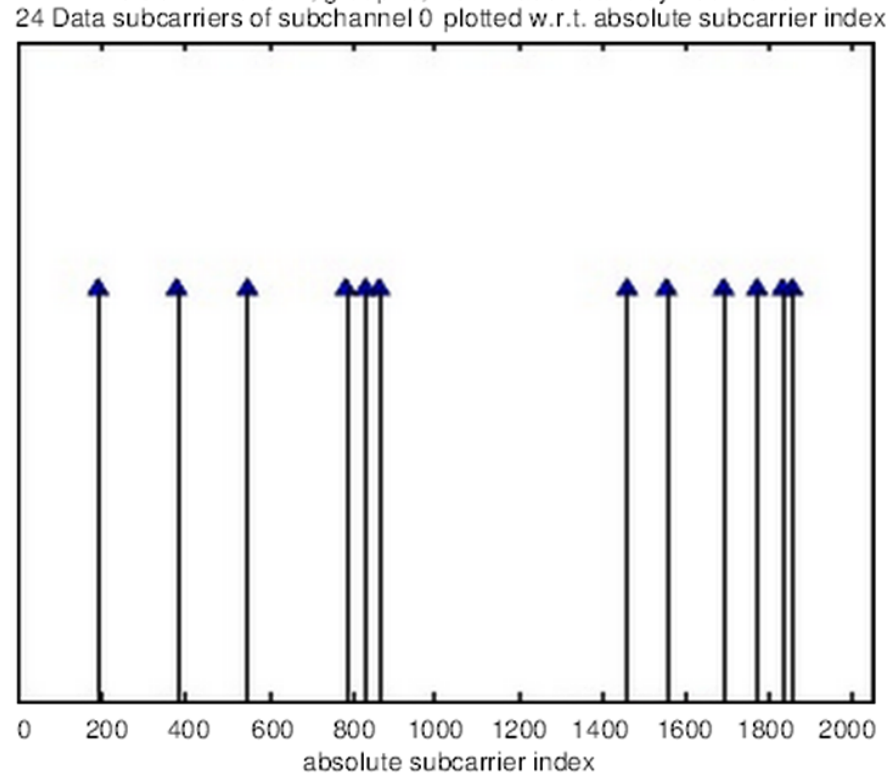

Figure 3.15: Subcarriers of subchannel plotted against absolute subcarrier index [from [104]].

1. The whole pool of channels and subchannels is available to all BSs in the network.

2. Each slot can be allocated to one user only within a cell, in order to avoid intracell interference and gain maximum achievable data-rate.

3. Neighbouring cells may reuse the same subchannels and channels if it does not cause mutual interference.

4. The transmission power on each subchannel is fixed, whereas the transmission rate is variable depending on the channel condition (SNR).

Assumption 2 is based on [89, 33]. It shows that an OFDM system can achieve the best performance when each channel is assigned only to one user in each cell. 


\begin{tabular}{|c|c|c|c|}
\hline OPT levels & BS power (dBm) & BS power $(\mathbf{W})$ & $\begin{array}{c}\text { Subchannel } \\
\text { power }(\mathbf{d B m})\end{array}$ \\
\hline 1 & 27 & 0.5 & 9 \\
2 & 29 & 0.79 & 11 \\
3 & 31 & 1.26 & 13 \\
4 & 33 & 2.0 & 15 \\
5 & 35 & 3.16 & 17 \\
6 & 37 & 5.01 & 19 \\
7 & 39 & 7.94 & 21 \\
8 & 41 & 12.6 & 23 \\
9 & 43 & 20.0 & 25 \\
10 & 45 & 31.6 & 27 \\
\hline
\end{tabular}

Table 3.6: Total BS and subchannel power levels

\subsubsection{Power allocation}

If deployed by the optimization framework, a BS transmits at a power level from a predefined set $B_{j-k}^{P}$. The breakdown for sector and subchannel power at each level is given in Table 3.6 where 'OPT levels' is the notation of power levels used in the optimization framework. The maximum power is limited by the regulatory body in each country (e.g. $45 \mathrm{dBm}$ in European countries).

In this thesis it is assumed that all the users within a cell receive the signal on the same transmit power level irrespective of the channel conditions.

\subsection{Interference calculation}

Interference evaluation of the system is a crucial stage of the network planning, as fully loaded wireless networks tend to be interference limited. There are numerous factors affecting interference [58] including: 
- Out of Block Emission

- Receiver selectivity

- Transmitter adjacent channel emissions

- Adjacent channel interference ratio

- Antenna characteristics

- Base station / subscriber station location

- Frequency reuse pattern

With universal frequency reuse in WiMAX systems the spectral efficiency near the cell boundaries is significantly reduced due to Inter-Cell Interference (ICI) There is number of ICI mitigation techniques discussed in the literature, namely interference avoidance (IA), interference randomization, interference cancellation, fractional frequency reuse and inter-sector coordination. WiMAX embeds some of these techniques, i.e. distributed permutations such as PUSC is a good example of IA in a mobile environment.

The main source of interference in a WiMAX network comes from subcarrier collisions within the selected slots of neighbouring cells. Therefore, average interference in a cell increases as the traffic load of other cells increase. The main task of the interference determination lies in finding the collision rates among the interfering cells.

By intelligently allocating slots to users the SINR ratio can be significantly improved, thus, increasing the achievable bit-rate. The interference power in a subchannel is linearly proportional to the number of subcarrier collisions in a subchannel.

Resources are allocated to users based on the periodic channel measurements and traffic demand. This information allows us to determine the interfering BSs and corresponding achievable rates. Therefore the SINR received by user $i, \gamma_{i}$, can be calculated according to the following expression: 


$$
\begin{gathered}
\gamma_{i}=\frac{F_{j-k}\left(R_{i}\right)}{\sum_{S_{y} \in S} \sum_{B_{x} \in B_{S_{y}}} I_{x-y}\left(R_{i}\right)+\Gamma_{i}} \\
\forall i: R_{i} \in R, R_{i}^{c o v}=1, F_{j-k}\left(R_{i}\right)=\operatorname{argmax}\left(F_{x-y}\left(R_{i}\right)\right) \\
\forall x, y: R_{i-x-y}^{c o v}=1
\end{gathered}
$$

where $F_{j-k}\left(R_{i}\right)$ is the received signal strength from the serving antenna $B_{j-k}, I_{x-y}\left(R_{i}\right)$ is the total interference level from the cell $B_{x-y}$ and $\Gamma_{i}$ is a noise. Conditions 3.5 and 3.6 show that the signal strength from the serving station must be the highest and the rest of the stations covering RTP $R_{i}$ and using the same slot are creating co-channel interference.

Noise can be calculated as:

$$
\begin{gathered}
\Gamma_{i}=\Gamma_{a m b}+\Gamma_{b k g} \\
\Gamma_{b k g}=\left(T_{r x}+T_{b k g}\right) \times b w \times \kappa \\
\Gamma_{a m b}=b w \times \sigma \\
T_{r x}=\left(N F_{W}-1.0\right) \times T_{b k g} \\
b w=N_{\text {subch_used }} * 20000000 / N_{\text {subch }}
\end{gathered}
$$

where $\Gamma_{a m b}$ and $\Gamma_{b k g}$ are the ambient and background noise levels respectively, $T_{r x}$ the receiver and $T_{b k g}=290 \mathrm{~K}$ the background temperatures, $\kappa$ is the Boltzmann constant (1.3806488E-23), $\sigma$ is the ambient noise level constant (1.0E-26), NF is the noise factor of the receiver $(4 \mathrm{~dB})$ and $N F_{W}$ is the value in Watts, bw is bandwidth (in $\mathrm{Hz}$ ) allocated to the user, $N_{\text {subch_used }}$ subchannels are allocated to a user and $N_{\text {subch }}$ is the total number of subchannels within a single time symbol.

$$
I_{x-y}\left(R_{i}\right)=\sum_{L_{s} \in L} I_{x-y}^{s}\left(R_{i}\right)
$$




$$
I_{x-y}^{s}\left(R_{i}\right)=\frac{\sum_{L_{w} \in L} \delta_{x-y}^{w} \digamma\left(B_{x-y}^{\text {PermBase }}, B_{j-k}^{\text {PermBase }}, s, w\right)}{\left(\sum_{L_{w} \in L} \delta_{x-y}^{w}\right) \times N_{\text {subcar }}} \times F_{x-y}\left(R_{i}\right)
$$

Equation 3.8 gives the expression for the total interference power from a particular cell. Whereas, expression 3.9 calculates interference from a cell on individual slots, where $\delta_{x-y}^{w}$ indicates if slot $L_{w}$ is in use in sector $B_{x-y}, \digamma($.$) is a function of four$ parameters to identify the number of subcarrier collisions in a pair of slots: $B^{\text {PermBase }}$ identifies the serving and interference cells, and slot indices respectively, $F_{x-y}\left(R_{i}\right)$ is the field strength of the interfering signal. Using the procedure of subchannels formation described in Section 3.5.3, $\digamma$ (.) gets the ratio of subcarriers in the given slots and PermBase parameters of the serving and interfering cells to the total number of subcarriers in these slots. The interference is therefore reduced according to the ratio of the collisions.

\subsection{Throughput estimation}

The theoretical throughput calculation for the DL subframe in Mobile WiMAX employing an OFDMA profile is done using the following expression:

$$
C_{\text {subfr }}^{D L}=\frac{N_{\text {symbols }} N_{\text {subcar }}}{t_{f r}}(\text { symbols/second })
$$

It accounts for the number of symbols used for data transmission in the DL subframe $N_{\text {symbols }}$, the number of data subcarriers in the frequency dimension $N_{\text {subcar }}$ and the duration of the frame $t_{f r}$. This gives the raw capacity but does not account for the signalling overheads. However, this issue is discussed in the section 3.8 .

Wireless networks in comparison to wired suffer from rapidly fluctuating channel conditions. This means that the system must be able dynamically adopt to changing conditions and control the power and coding rate in order to constantly maintain the highest 
channel capacity at all times. The use of the advanced modulation and coding schemes has a significant impact on the raw capacity of the channel. The uncoded channel size varies by a factor of nine between the highest and lowest modulations. This means that depending on the channel conditions the capacity can vary by nearly an order of magnitude.

Based on the adoption of AMC schemes for varying SINR levels of the channels, the throughput calculation of the WiMAX system can be described as a ring structure. The further a user is located from the cell the lower its SINR level and as a result the achievable bit-rate is reduced. Hence, the actual mapping between the SINR measures and the achievable rates is a function of fading profiles and the available modulation/coding techniques. Examples of the rate functions in terms of SINR can be found in [153, 44]. The theoretical throughput calculation per modulation scheme for DL subframe is expressed as follows:

$$
C_{\text {subfr }}^{D L}=\frac{b_{m} c_{r} N_{\text {symbols }} N_{\text {subcar }}}{t_{f r}}(b p s)
$$

where $b_{m}$ is the modulation gain, which is 2 for QPSK, 4 for 16-QAM, and 6 for 64-QAM; $c_{r}$ is the coding rate of the chosen modulation.

Given that the SINR level modulation and coding scheme can be identified, then this in turn can be matched with the maximum achievable bit-rate of the transmission. The SINR versus modulation values used in this thesis are presented in Table 3.7 The switching process between modulations is represented by transition thresholds SINR MIN and SINR MAX - when a threshold is reached, the modulation order changes until another threshold is achieved. The definitions of the thresholds may vary depending on the goal of the adaptation (switching) algorithm - minimizing the error probability or maximizing throughput.

As previously defined the minimum allocation unit referring to the smallest two-dimensional quantum, for users is a slot. By means of CSI and resource allocation algorithms em- 


\begin{tabular}{cccc}
\hline MODULATION & CODING RATE & SINR MIN $(\mathrm{dB})$ & SINR MAX $(\mathrm{dB})$ \\
\hline QPSK & $1 / 2$ & 0 & 5 \\
\multirow{2}{*}{ 16QAM } & $3 / 4$ & 5 & 12 \\
& $1 / 2$ & 12 & 19 \\
64QAM & $3 / 4$ & 19 & 23 \\
& $1 / 2$ & 23 & 25 \\
& $2 / 3$ & 25 & 27 \\
& $3 / 4$ & 27 & 29 \\
& $5 / 6$ & 29 & $>29$ \\
\hline
\end{tabular}

Table 3.7: SNR thresholds based on modulation and coding rate

bedded in WiMAX, the slots are chosen for each user to maximize overall capacity. A slot capacity can be expressed as a fraction of the subframe capacity:

$$
C_{\text {slot }}^{D L}=\frac{C_{\text {subfr }}^{D L}}{N_{\text {slots }}}=\frac{N_{\text {smb_slot }} b_{m} c_{r} N_{\text {subcar }}}{t_{f r} N_{\text {subch }}}(\text { bps })
$$

where $N_{\text {slots }}$ is the total number of slots in the DL subframe which can be defined as $\frac{N_{\text {symbols }} N_{\text {subch }}}{N_{\text {smb_slot }}}$, and $N_{\text {smb_slot }}$ is the number of symbols per slot (1, 2, or 3 symbols), a partitioning parameter for different permutation schemes which is presented in Table 3.4

Given the transmission bit-rate of an assigned slot it can be calculated whether more slots need to be assigned to a user in order to satisfy its bit-rate demand.

\subsection{Transmission overheads and cell capacity}

To analyse the actual capacity of $802.16 \mathrm{e}$ WiMAX radio, the raw capacity needs to be adjusted based on the amount of signalling overheads. The main parameters of OFDMA PHY are summarized in Table 3.8, In this section the overview of DL overheads analysis and the formulation of capacity is presented. 


\begin{tabular}{|l|c|c|c|}
\hline Parameter & \multicolumn{3}{|c|}{ Values } \\
\hline System Bandwidth & 5 & 10 & 20 \\
\hline FFT size & 512 & 1024 & 2048 \\
\hline Total OFDMA symbol duration $(\mu \mathrm{s})$ & \multicolumn{3}{|c|}{100.84} \\
\hline Frame duration $(\mathrm{ms})$ & \multicolumn{3}{|c|}{5} \\
\hline Symbols/frame & \multicolumn{3}{|c|}{49} \\
\hline Data symbols & \multicolumn{3}{|c|}{44} \\
\hline
\end{tabular}

Table 3.8: Mobile WiMAX PHY parameters

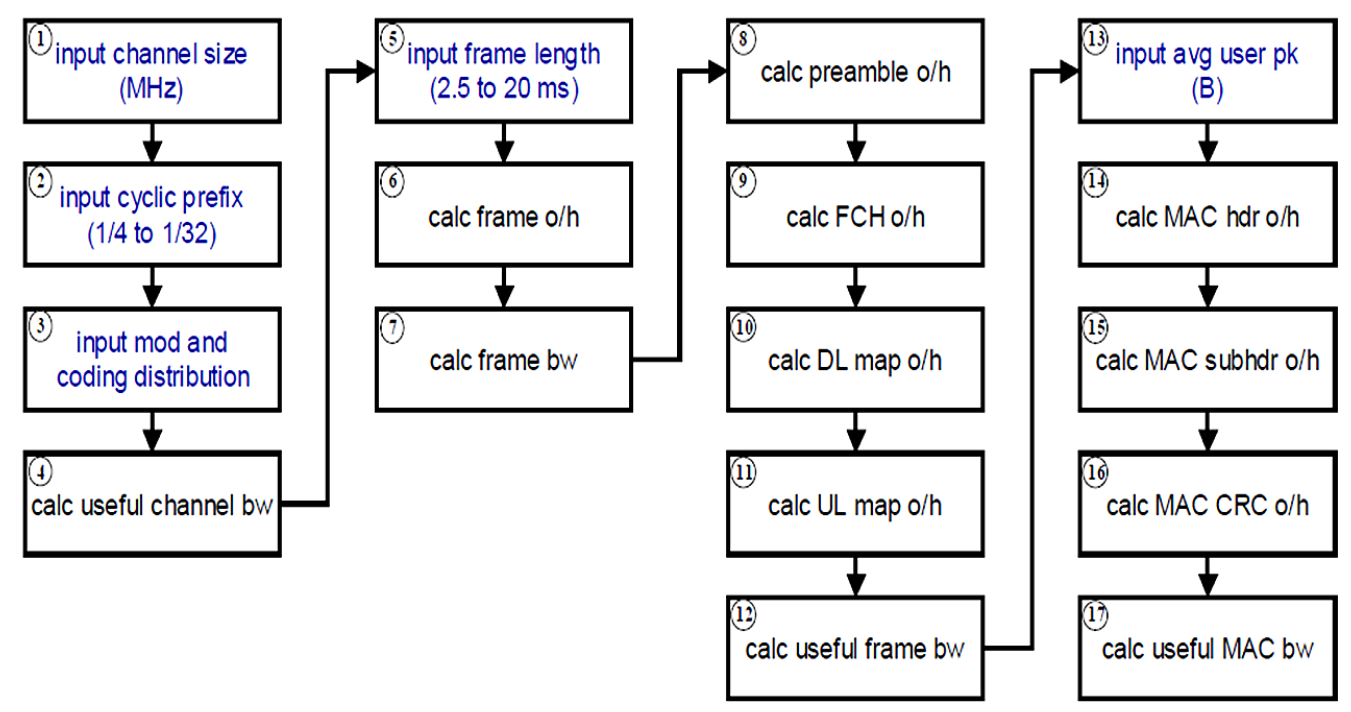

Figure 3.16: Downlink bandwidth calculation

There are numerous complex factors in the WiMAX MAC and PHY layers which affect the base station capacity estimation. A step-by-step approach with consideration of all the major overheads and the process of calculating the useful channel bandwidth can found in [5]. Figure 3.16 depicts this process with the account of the various PHY and MAC overhead contributions. These contributions are different in nature - the PHY layer introduces fixed overheads, whereas the MAC layer is more variable and depends on configurations of the system and traffic type.

The overhead elements can be described as follows: 
- Incomplete symbols

- DL preamble

- Frame Control Header (FCH)

- UL/DL MAP

- MAC protocol data units (MAC-PDU)

- Data burst

Considering the listed overheads the effective capacity is reduced by: the preamble length (2 OFDM symbols), FCH message length (1 OFDM symbol), and broadcast messages (DL-MAP, UL-MAP, UCD, DCD) where length is variable according to the number of served users in the cell, as well as to the length of control messages specified to each user.

In depth analysis of each element is not considered here. However, the explicit calculations of the overheads are presented in [125, 41, 5, 31, 113].

The following assumptions are used in this thesis:

- The signalling overhead in DL subframe encounters of $24 \%$ of the PHY capacity, indicated in the studies [82].

- The frame is partitioned to downlink and uplink subframes with a DL/UL ratio set of $3: 1$, with 37 symbols allocated to DL and 12 to UL.

- Removing overheads from the DL leaves 28 symbols for the actual capacity.

$$
B_{j-k}^{\text {cap }}=N_{\text {slots }}\left(1-r_{\text {overheads }}\right)
$$

The capacity of the base station sector, denoted as $B^{c a p}$, is derived from the number of slots available in the channel within a frame with removed overheads $r_{\text {overheads }}$. Having assigned a number of slots to a user the capacity is reduced by this number of slots. 
Chapter 4

\section{Optimization - General Framework}

This section addresses the problems of automatic cell planning and automatic frequency planning and solving these problems using an optimization framework first in a traditional way when they are considered separately and then in the more complex combined fashion to address the two fundamental network design problems simultaneously.

\subsection{Optimization framework background}

It has been shown that wireless network problem belongs to the class of NP-hard problem [52, 25, 103]. This implies that an exhaustive search, where every possible solution is evaluated in order to obtain the optimal solution is unacceptable strategy for the real world problems as the size of the problems tend to grow very large very quickly. When faced with this kind of problem an approach needs to be employed which allows to obtain high-quality solutions in a reasonable time. Such an approach is called heuristic. The following definition provided by Reeves in 1996 gives a good description:

A heuristic technique (or simply heuristic) is a method which seeks good (i.e. nearoptimal) solutions at a reasonable computation cost without being able to guarantee optimality, and possibly not feasibility. Unfortunately, it may not even be possible to state how close to optimality a particular heuristic solution is. 
Meta-heuristic techniques have become a common approach to solve problem of planning cellular/wireless networks with the following most popular methods:

- Simulated annealing (SA),

- Genetic algorithms (GA),

- Tabu search (TS).

Genetic algorithms are search methods based on principles of natural selection and genetics [63, 35, 79]. GAs encode the decision variables of a search problem into finite-length strings of alphabets of certain cardinality. The strings which are candidate solutions to the search problem are referred to as chromosomes, the alphabets are referred to as genes and the values of genes are called alleles. In contrast to traditional optimization techniques, GAs work with coding of parameters, rather than the parameters themselves. Another important concept of GAs is the notion of population. Unlike traditional serach methods, genetic algorithms rely on a population of candidate solutions. The population size, which is usually a user-specified parameter, is one of the important factors affecting the scalability and performance of genetic algorithms. The main concept of GA is based upon the concept of survival of the fittest. Starting with a population of randomly generated solutions through breeding, mutation and death the overall fitness of each solution is raised over successive generations [68]. There are several studies concentrating on using GAs to obtain solutions for cellular planning problems [38, 109, 37, 154, 77, 88].

Tabu search was proposed by Fred Glover in 1986 to allow hill climbing to overcome local optima [65, 66, 67]. The basic principle of tabu search is to pursue the search whenever a local optimum is encountered by allowing non-improving moves; cycling back to previously visited solutions is prevented by the use of memories, called tabu lists, that record the recent history of the search. Tabus are one of the distinctive elements of tabu search when compared to hill climbing. As already mentioned, tabus are used to prevent cycling when moving away from local optima through non-improving 
moves. The key realization here is that when this situation occurs, something needs to be done to prevent the search from tracking back its steps to where it came from. This is achieved by making certain actions tabu. This might mean not allowing the search to return to a recently visited point in the search space or not allowing a recent move to be reversed. Tabus are stored in a short-term memory of the search (the tabu list) and usually only a fixed and fairly limited quantity of information is recorded. The TS heuristic was applied to the antenna placement problem as well as to the frequency assignment problem in [136, 93, 123, 84, 49, 24, 96].

Simulated annealing is a heuristic optimization algorithm which is based and inspired by an analogy between the physical annealing process of solids and the problem of solving large combinatorial optimization problems. In condensed matter physics, annealing is known as a thermal process for obtaining low energy states of a solid in a heat bath. The process consists of the following two steps [91]: increase the temperature of the bath to a maximum value at which the solid melts; decrease carefully the temperature of the heat bath until the particles arrange themselves in the ground state of the solid. A typical feature os SA is that, besides accepting improvements in cost, it also accepts deteriorations to a limited extent. Initially, at large values of temperature, large deteriorations will be accepted; as temperature decreases, only smaller deteriorations will be accepted and finally, as the value approaches 0 , no deteriorations will be accepted at all. This feature means that SA, in contrast to iterative improvement, can escape from local minima while it still exhibits the favorable features of iterative improvement, i.e simplicity and general applicability. Simulated annealing has been widely used within the area of cellular network planning [101, 64, 134, 28, 17, 105, 83].

It can be seen that all the listed techniques have been successfully used to tackle the fundamental problems of cellular network planning and frequency planning. With no theoretical prove of one method being superior to another, that is also confirmed in No Free Lunch theorem [147] which states: 'For all possible performance measures, no search algorithm is better than another when its performance is averaged over all 
possible discrete functions', the following criteria can be considered when selecting the optimization technique for network planning: the objectives of network planning, techniques employed in the framework, the required quality of solution, computation time and other technique-specific considerations. Technique-specific considerations for the three algorithms are as follows:

\section{Genetic algorithms}

- representation is crucial, complex data structures for the specific problem must be determined;

- effectiveness could be sensitive to choice of parameter values and operators, the large number of parameters must be set through a lot of experimentation in order to acquire solutions of the required quality.

\section{Tabu search}

- effectiveness depends on strategy of tabu-list manipulation, definition of the search space and the neighbourhood structure.

\section{Simulated annealing}

- neighbourhood function is crucial to its effectiveness;

- cooling schedule could be important.

As described, all the techniques have their advantages and disadvantages in the form of complexity of the implementation. The main goal of the research work presented in this thesis was not to compare the effectiveness of different optimization methodologies but to develop a model and a framework for planning Mobile WiMAX networks optimizing all the given objectives. The simulated annealing has been chosen as a foundation of the optimization work due to the following elements and characteristics: performance, i.e running time and solution quality; ease of implementation; and 
applicability and flexibility. The SA is conceptually very simple and quite easy to implement. With respect to applicability and flexibility it has become obvious as a result of the overwhelming amount of practical experience that has been gathered over the past 30 years that simulated annealing can be considered as one of the most flexible and applicable algorithms that exist. With respect to performance, the solution quality can be typically traded against running time. Despite numerous studies it is still difficult to judge SA on its true merits. Another justification for choosing SA is that it can be more efficient than GA and TS because of the single configuration - it can be made more efficient to update the configuration after a single change has been made. This applies to GA's but is required for all the population members, for TS the whole neighbourhood needs to be evaluated. SA is the only method that updates a single network configuration.

The optimization framework is based on a Simulated Annealing (SA) algorithm [91] powered by a range of neighbourhood moves. SA is a generic probabilistic metaheuristic for locating a good approximation to global optimum of a function in a large search space. As it incorporates an iterative process, at each step some small changes are made to the current solution to explore and evaluate neighbours. These moves can be divided on two categories: random - which help to explore new areas of the search space and to avoid getting stuck in a local optimum, and intelligent moves which specifically target specific network deficiencies. The assessment and evaluation of new network designs is based on a weighted objective function. When new solutions yield better values they are always accepted, otherwise the algorithm probabilistically decides whether to move to a new state. This mechanism helps to avoid the algorithm converging to solutions which are suboptimal.

The overall framework is depicted on Figure 4.1 . 


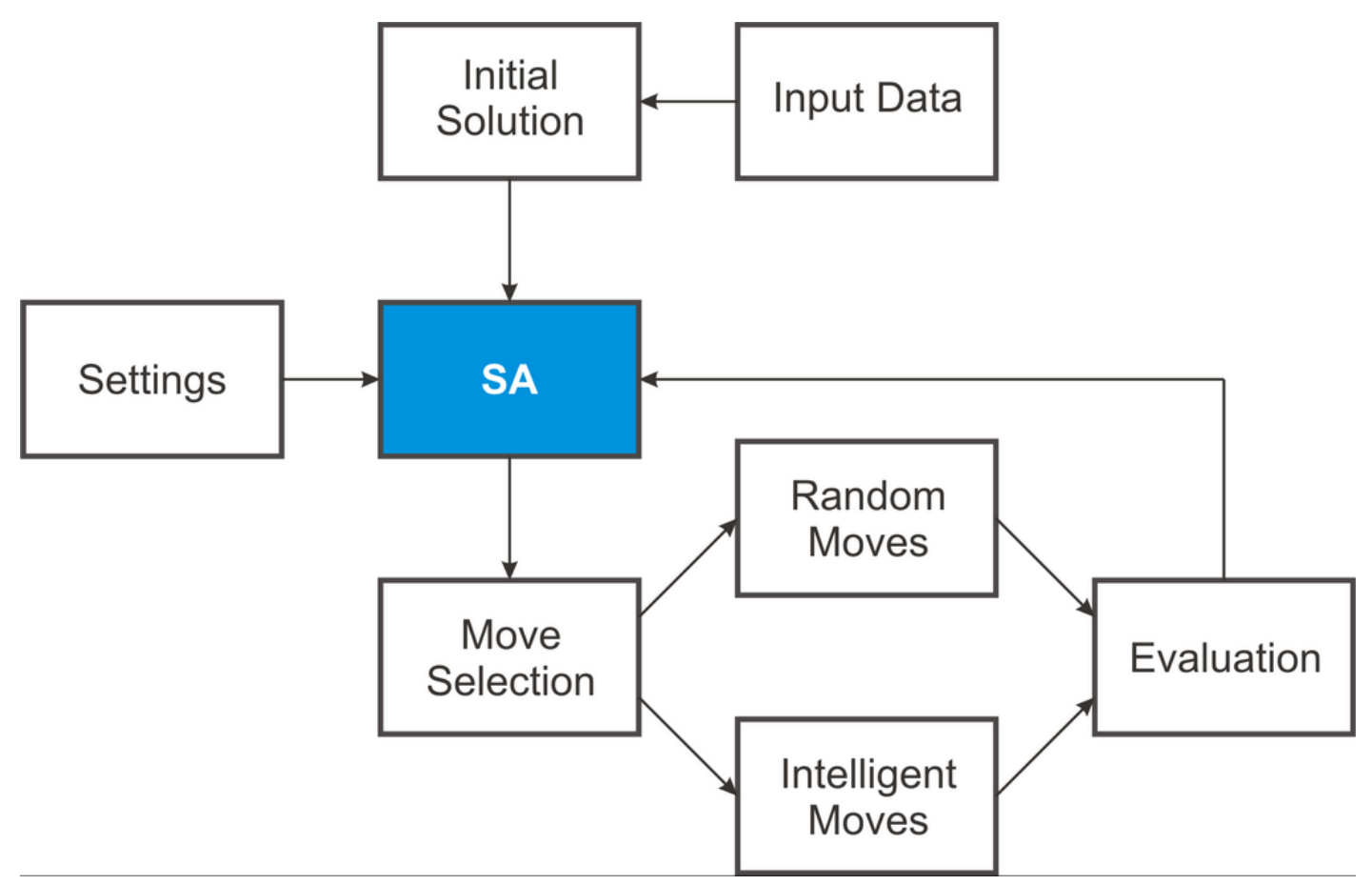

Figure 4.1: Optimization framework

\subsubsection{Input data}

Accuracy of input data has a significant effect on the quality of the final network designs. Prior to the planning stage, network operators spend a considerable amount of time to prepare all the required information for the desired area. This data includes: geographical positions of potential sites; distribution of RTPs within the area; propagation model or measurements for all RTPs; distribution of users at so-called peak hours (when the network is loaded the most); traffic model, which represents throughput and QoS demands for the users; the financial model reflecting the capital and operational costs for running the network and predictions of revenues and profits.

\subsubsection{Initial network}

One can choose the initial state starting point randomly, and begin annealing at a sufficiently high temperature that most moves are accepted. Alternatively, a fast problem- 
specific heuristic is used to find an initial state with a moderately small cost function value, and annealing begins at a lower temperature.

The initial network is a starting point for the algorithm. There are several scenarios for building it depending on any pre-existing infrastructure. An existing network can be used as the initial one if it is to be expanded or reorganised. Otherwise, when the service provider is deploying a new network it can be produced from the following options:

- random - select a random number of sites from the set of potential ones and activate with maximum number of BSs randomly configured.

- random with full coverage - activate sites in a random order until the whole network is covered

\subsubsection{Simulated Annealing configurations}

Simulated annealing is not without its critics and possesses some positive and negative features. The strongest advantage over the range of available techniques is a statistical guarantee of finding an optimal solution [91]. Furthermore, SA can:

- process cost functions possessing quite arbitrary degrees of non-linearities, discontinuities, and stochasticity;

- process quite arbitrary boundary conditions and constraints imposed on these cost functions;

- be easily implemented compared to other optimization algorithms;

Critical points of SA include:

- too costly in terms of computational run-time in order to find an optimal solution (main criticism); 
- complex in tuning the algorithm for specific problems (compared to other techniques);

- misused by transforming SA into SA-like techniques, e.g. simulated quenching (SQ), which do not predict the optimality, but nonetheless are powerful tools;

- mainly based on physical properties rather than on mathematical ideas.

The original Metropolis Monte Carlo algorithm [107] was generalized by Kirkpatrick who included a temperature schedule to improve the efficiency of the algorithm [91].

We do not seek the guarantee of finding global optima but a good solution in a reasonable time. With the ease of implementation of SA some modifications have been applied to the standard simulated annealing (Boltzmann annealing [128]) in order to speed up the optimization process. The general structure of the SA is presented in Algorithm 4.1.

The idea behind the algorithm is to heat the system to some initial high temperature and then slowly cool it down. This is called annealing, which lets the system settle into a low energy state without getting stuck in a local minima. The analogy is taken from the physical systems in order to solve combinatorial optimization problems: the cost function is associated with the energy of a physical system; the solution space is associated with the state space. The search space of the problem is probed by a probabilistic hill climbing search, which is controlled by a parameter $T$ that plays the role of the temperature in a physical system. By gradually and slowly decreasing $T$ towards zero according to a chosen schedule, it can be shown that the globally optimal solutions are approached asymptotically. By randomly proposing a move from a set of predefined moves a new state is formed. A move is defined as a perturbation whose utilization to the current state leads to a new state. The move selection process is called in this work as move generation strategy and fully described in the following section. Then it is decided if the proposed move is accepted or rejected by probability $p$. If a proposed move is accepted, the new state becomes the current state; otherwise 


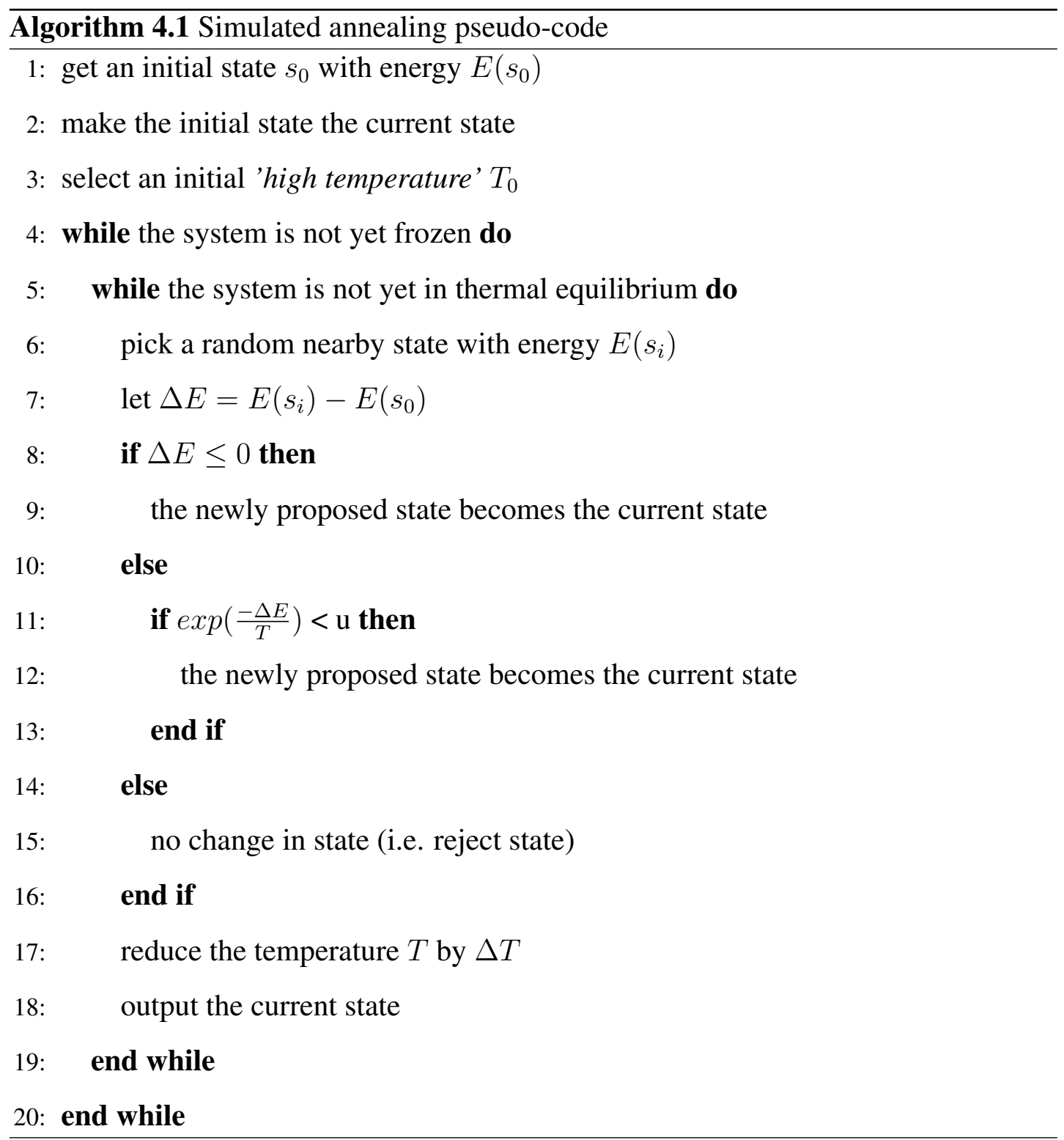

the current state remains unchanged. The probability of accepting an uphill move (which results in a positive $\Delta E$ ) is controlled by the temperature $T$. This gives to SA a mechanism to widely explore the state space. The process of exploration is repeated until the system reaches the state of thermal equilibrium. Then, the temperature is reduced according to a schedule and the system is allowed to reach thermal equilibrium ones again. The process is terminated when no significant improvement is expected by further lowering the temperature. 
The main issues with the implementation of SA include the problem-specific choices such as objective function, neighbourhood and constraints, and generic settings - generation and acceptance probabilities and the cooling schedule.

Acceptance Probability Function: The probability of moving from the current solution state $s$ to a new state $s^{\prime}$ is defined by the function $p\left(E(s), E\left(s^{\prime}\right), T\right)$, where $E(s)$ and $E\left(s^{\prime}\right)$ are the energies (cost) of the current and new state respectively, and $T$ is the temperature. There is a considerable amount of experimentation approaches presented in the literature on the acceptance probability but we employ the standard and the most common - exponential function [15]. The probability function $p$ is required to be non-zero when $E\left(s^{\prime}\right)>E(s)$, meaning that there is a chance of a system being in a high energy state, in other words accepting worse solutions (uphill moves) in order to avoid getting stuck in local minimum in favour of finding a global solution. The transformation of the state $s$ highly depends on the temperature $T$, i.e. the chances of accepting uphill moves are diminishing with the reduction of the temperature. We use the so-called Boltzmann probability distribution:

$$
p\left(E(s), E\left(s^{\prime}\right), T\right)=\exp \left(-\frac{E\left(s^{\prime}\right)-E(s)}{T}\right)
$$

Cooling Schedule: defines the initial temperature, procedure for changing the temperature during the run, and stopping criterion. As Romeo and Sangiovanni-Vincentelli state in their work [121] - an effective scheduling is essential to reduce the time for the algorithm to converge to an optimal solution. Cooling schedules are heuristic in nature, striving to balance the reasonable run time with SA dependence on asymptotic behaviour. We adopt the static schedule representation which implies the complete identification of one prior to the beginning of the algorithm. That is contrary to the adaptive approach when the temperature is adjusted dynamically based on the information obtained during the execution. Many approaches and heuristic methods have been presented in the literature to find the optimal cooling schedule. Full analysis can be found in [42]. However, these schedules fully depend on the chosen neighbourhood 
and the topology of the objective function and their effectiveness can only be compared through experimentation.

As the scheduler used in this SA equation 4.2 is shorter than those guaranteeing the convergence to global minimum, a record of best performing solutions found during the optimization are maintained, as when the algorithm terminates the last found solution may not be the best.

$$
T_{k+1}=a T_{k}, \quad 0<a<1
$$

\section{Initial temperature}

The initial stage of simulated annealing is generally directed to global searching to investigate alternative optima. It is important to identify the sensitivity of the global exploration to the initial temperature. This can improve the efficiency of the SA. For many problems much time is spent inefficiently until an annealing temperature is reached which starts to effectively sample the search space and getting to a local minima. One solution has been proposed in [32] where some short sample runs were performed to determine this effective temperature. Their numerical experiments indicate that for SA optimization one should spend most of the time determining $T_{0}$. Then conventional slow cooling schedule can be replaced by a schedule with rapid cooling. We choose the initial temperature such that after one loop of the given temperature the algorithm has a $40 \%$ acceptance ratio.

\section{Stopping criteria}

It has become successful and popular in stopping SA at low temperatures when some low predetermined acceptance ratio is reached. Acceptance criteria are biased according to information being gathered on the cost function during the search, maintaining detailed balance throughout the search. We suggest using SA until some low acceptance criteria is reached and then terminate the algorithm. This ratio is chosen in such 
a way that when the system approaches its frozen state, the decrement of the average energy from one temperature to the other is small.

\subsubsection{Move generation strategy}

The choice of move generation strategy highly influences the performance of simulated annealing. As previously mentioned, the Metropolis method has one major drawback - the run-time is quite high because many candidate solutions are rejected at low temperatures. Moreover, when heuristics are used to build a good initial state, annealing starts at a low temperature and as a consequence there is low acceptance rate in the following runs. The low acceptance rate is necessary as this method does not maintain information about the effect of the possible moves on the cost function. The algorithm must uniformly choose from the set of $N_{\text {moves }}$ possible moves and then reject most selections.

The alternative is to maintain the effects of each possible move on the cost function and use this information to bias the selection of moves. The move generation probability function used in this work is not a temperature-dependent distribution with probability proportional to the neighbourhood size. Since the main drawback of simulated annealing is its excessive run-time we introduce a modification to the generation function to speed up the algorithm. The increase in speed is achieved by decreasing generality. Instead of the standard approach which blindly generates neighbours uniformly, we adopt an intelligent mechanism which modifies its probability distribution dynamically to accommodate search intensification and diversification. The idea is adopted from [132, 61] where Tovey suggests an approach called neighbourhood prejudice swindle. Similarly, a very methodical approach called rejectionless was developed in [74] yielding a search time not dependant on the acceptance ratio or temperature.

Prior to the start of the optimization process all the moves from the neighbourhood set are equally weighed with the same probabilities of selection to allow the SA to explore 
the full range of modifications. However, as the search proceeds the algorithm dynamically evaluates the acceptance ratio of moves (number of accepted to the total number) within a fixed number of iterations. Then SA dynamically corrects the corresponding weights according to the performance of each move, so that the most accepted moves are executed more frequently in the following loop.

\section{Move selection strategy}

A set of moves defined for each optimization stage is used to steer the initial stage into making changes to the configurations of base stations and network which are deemed to be problematic. This selection procedure has an important impact on the search progress and final result. We introduce a mechanism (probabilistic by nature) to control and target these modifications towards deficiencies in the network as efficiently as possible.

There are two ways in making modifications - randomly selecting the sites and configurations for the base stations, or intelligently, which are performed according to the performance of certain moves. This means that the best performing moves are selected more frequently, thus improving problematic base stations which are under performing in some way or another. For example, if a BS is not efficiently loaded then its configurations may be altered towards increasing the load by increasing power or tilting. Also the proportion between random and intelligent moves plays a significant impact on the advancement along the search space. Having random moves is beneficial in terms of exploring new areas of the solution space, but too frequent use may slow down the progress and reduce the chances of converging to a good solution. The dominance of the intelligent moves may lock the search in the local optima, but it speeds up the progress by modifying and improving problematic areas of the network. Clearly some balance should be introduced between the two.

Definition 4.1.1. Acceptance ratio: is a rate between the accepted and the total number of perturbations for a particular move. 
One way of realizing this is to fix the probabilities of selecting moves equally, so that all the modifiers have the same chance of being chosen. However, this method has proved to be wasteful towards the end of annealing when the acceptance ratio of some moves drops dramatically. Having identified this trend the selection procedure has been modified to dynamically evaluate the acceptance ratio of the moves and giving priorities to those performing the best. The pseudo-code of this strategy is given in Algorithm 4.2 .

The strategy is straightforward. Initially all the moves are equally weighted. In our case for Stage 1 of the optimization framework (section 5) four moves have been constructed, all with the weight of 0.25 (or $25 \%$ chance of being chosen). With every iteration of the Initial Coverage stage the number of accepted and the total number of moves are maintained. After 100 iterations of SA the acceptance ratio of all moves is retrieved and is expressed as the ratio of accepted number of moves (being selected and accepted for meeting the criteria) to a total number of moves (including rejected ones). Next, the moves are arranged in the increasing order of acceptance ratio with the lowest first. Finally the new weights are calculated.

To illustrate this: after first 100 iterations - or 100 moves being performed, say the following values have been derived:

- move site - 22 accepted, 26 total - acceptance ratio $\frac{22}{26} \approx 0.846$

- change power - 14 accepted, 25 total - acceptance ratio $\frac{14}{25}=0.56$

- change tilt - 17 accepted, 23 total - acceptance ratio $\frac{17}{23} \approx 0.739$

- site deactivation - 9 accepted, 26 total - acceptance ratio $\frac{9}{26} \approx 0.346$

It is clear that the 'move site' modifier is the most successful with more than $84 \%$ acceptance ratio, followed by 'change tilt' etc. After sorting the moves the new weights will be as follows: 


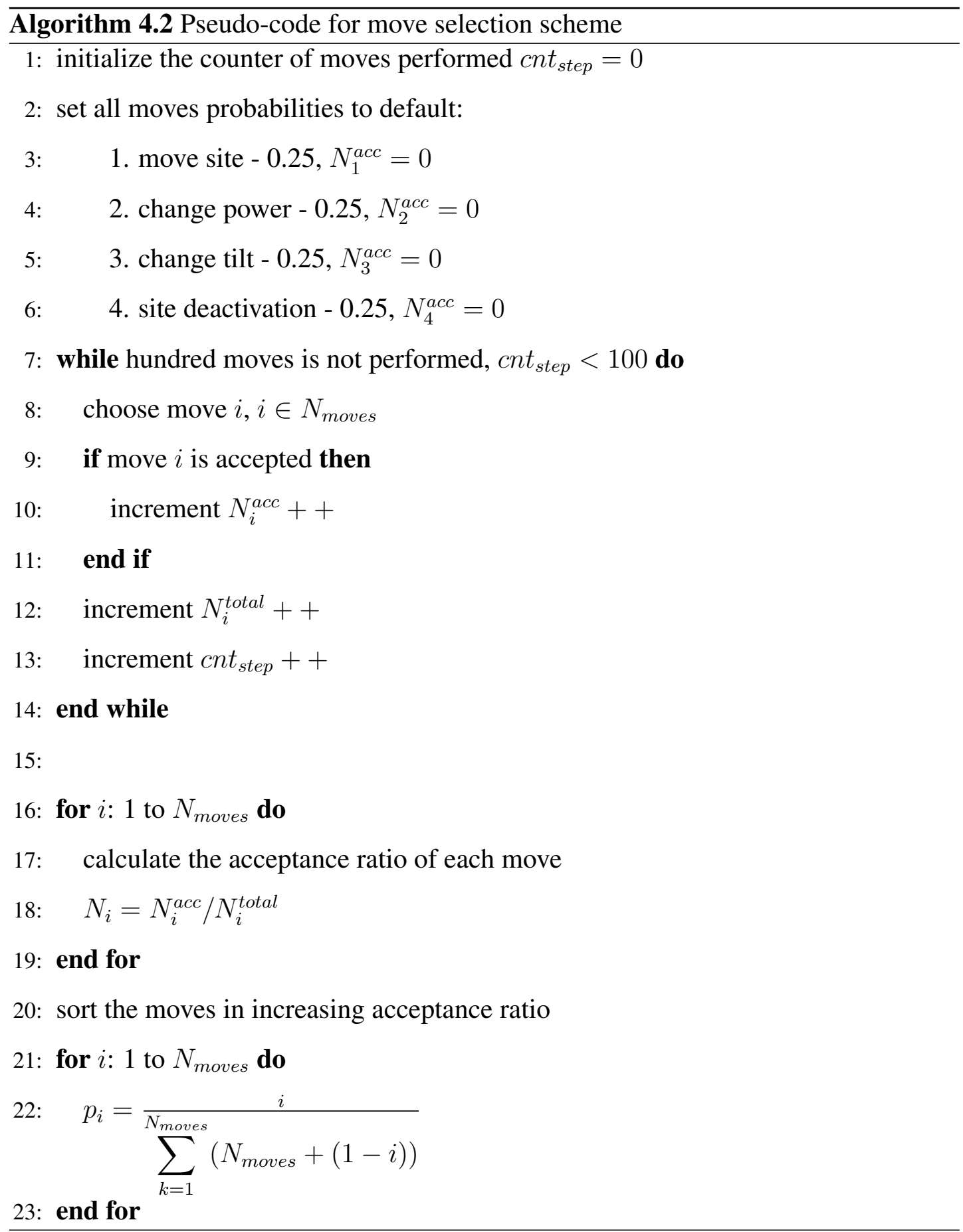

- site deactivation $-p_{1}=\frac{1}{4+3+2+1}=0.1$

- change power $-p_{2}=\frac{2}{4+3+2+1}=0.2$

- change tilt $-p_{3}=\frac{3}{4+3+2+1}=0.3$ 
- move site $-p_{4}=\frac{4}{4+3+2+1}=0.4$

Having identified the new priority levels for the moves in the next 100 iterations the choice will be governed by a randomly generated number $r n d$ between 0,1 and procedure presented in Algorithm 4.3:

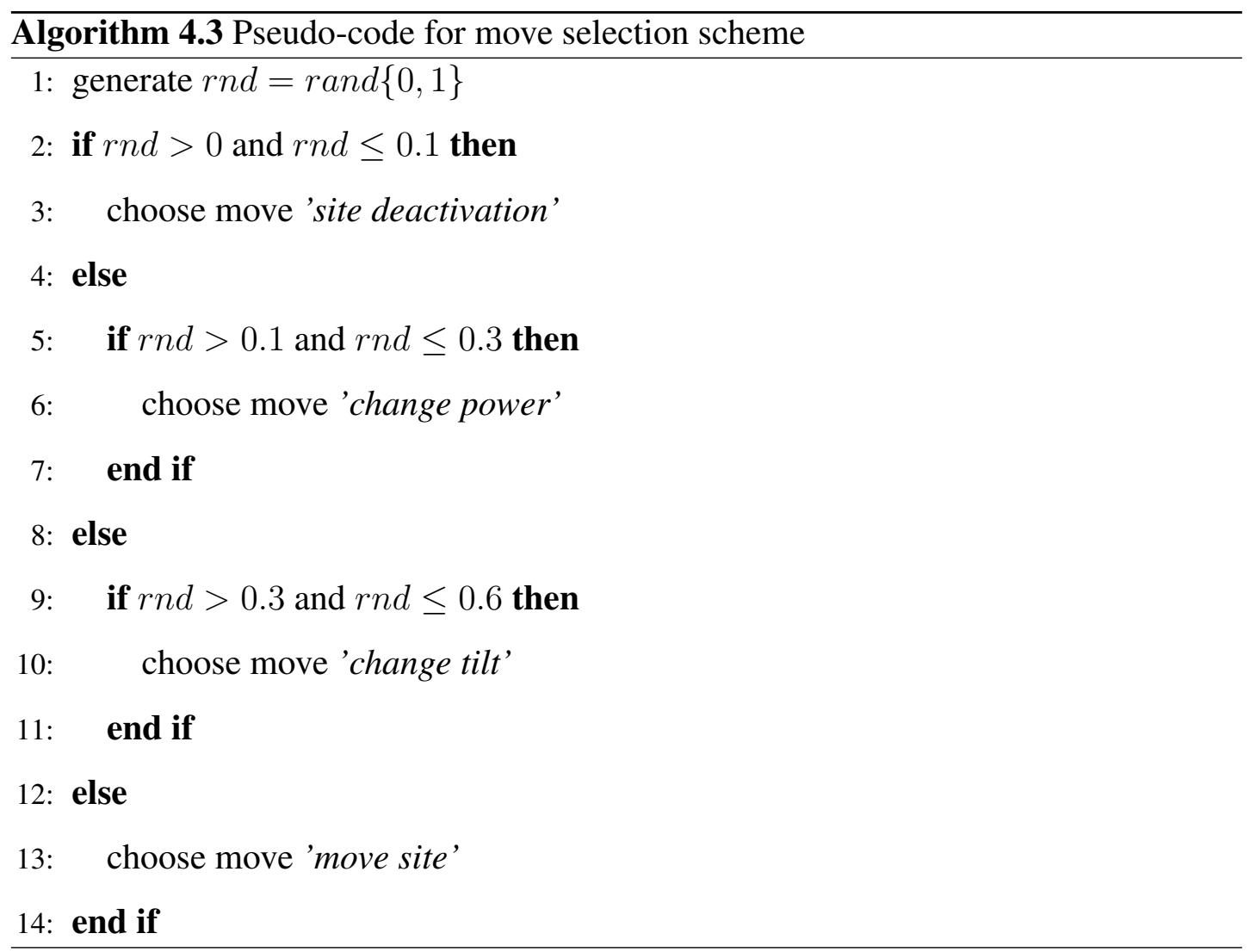

Therefore after each set of 100 iterations the performance of the moves is re-evaluated.

\subsubsection{Network objectives and cost function}

Multi-objective optimization is the process of simultaneously optimizing (finding a trade-off between) multiple conflicting objectives, subject to given constraints. As these objectives are competing it is no longer possible to find the single solution which is superior to all others in every objective. There are two approaches dealing with this: 
finding a number of solutions for which there exists no superior solutions - the Pareto set [47]. This set represents the optimal trade-off between objectives available - a set of solutions, none of which dominate each other (mutually non-dominating) and for which no feasible configurations dominate them.

Simulated annealing is a highly desirable algorithm for the problems with a single objective as there exists a theoretical proof that is able to show convergence to the optimal solution. Therefore we make use of a standard method of finding a solution to multi-objective optimization problem - single aggregate objective function (AOF). The idea behind AOF is to join all objective functions into a single fitness function by means of a weighted sum.

$$
E(s)=\sum_{i=1}^{N} w_{i} E_{i}(s)
$$

Here $E(s)$ is a combination of the $N$ objectives $E_{i}(s)$, with weights $w_{1} \ldots w_{N}$. Using this approach, the $E(s)$ function is to be minimized for $s \in \Omega$, where $\Omega$ is the solution space.

The use of $\mathrm{AOF}$ is attractive as the minimization of $E(s)$ gives a solution, the image of which in objective space lies on the Pareto set $x \in \Omega$. Also by varying the weights it is possible to locate different regions of the Pareto set. However, it is argued that the weighted sum approach is subjective in that a network designer must choose the weights. Despite the problems with the AOF approach, it has been shown in [81] that algorithms employing this technique can perform well, especially with a large number of objectives. This method has been adopted and modified by many [122, 135, 112].

From the view of an operator when building a network from nothing the planner must consider a large range of targets as well as limitations. He needs to ensure that the broadband service advances to market quickly, becomes profitable by optimizing CAPEX, not over engineering the network and provide a possible path for future growth. After the network is deployed, it should be built in a way for the operator to 
easily manage, tune and grow the system when required.

The initial investment for a WiMAX license is considerable and therefore the return is desired as quickly as possible. The most important element of commercializing the network is thorough planning both for the business model and in the operational network plan.

To plan and place a robust WiMAX network as quickly and cost-effectively as possible it is crucial to set the right objectives so the operator can focus on attracting customers with new wireless broadband solutions. Three objectives are presented all aiding the algorithm to find a profitable and effective final network.

\section{COVERAGE}

Coverage of the network is to be maximized, i.e. the number of RTPs receiving signal above service level threshold from at least one active base station:

$$
\begin{aligned}
& E_{1}=1-\frac{\sum_{i=0}^{|R|} R_{i}^{c o v}}{|R|} \longrightarrow M I N \\
& \forall R_{i}: \sum_{B_{j} \in B_{S_{k}}} \sum_{S_{k} \in S} R_{i-j-k}^{c o v} \geq 1 \\
& \frac{\sum_{i=0}^{|R|} R_{i}^{c o v}}{|R|} \geq \lambda^{c o v}
\end{aligned}
$$

Constraint 4.5 ensures that a RTP is covered if it receives signal from at least one base station. (4.6) represents the constraint on coverage to be achieved at least for $\lambda^{\text {cov }}$ percentage of users, imposed by service providers and is normally in the range of $90 \%$.

In [143] authors have introduced a method of determining and analysing the cost implications of providing different levels of service coverage in terms of a marginal cost 
measure. It has been shown that as the coverage level rises beyond some threshold there is a substantial increase in cost, relative to increase in service coverage, for additional provision. Although this threshold level is problem specific, this investigation shows that coverage above $95 \%$ is significantly more expensive.

\section{SERVICE}

Maximize service level, i.e. the number of users located at RTPs with satisfied service demand. This objective is to be optimized for the so-called peak hour when the traffic load in the network at its maximum:

$$
\begin{gathered}
\sum_{i=0}^{|R|} R_{i}^{\text {serv }} \\
E_{2}=1-\frac{M \mid}{|R|} \longrightarrow M I N \\
R_{i-j-k}^{\text {serv }} \leq z_{j-k}: \forall S_{k} \in S, B_{j} \in B_{S_{k}} \\
\sum_{B_{j} \in B_{S_{k}}} \sum_{S_{k} \in S} R_{i-j-k}^{\text {serv }} \leq 1: \forall R_{i} \in R
\end{gathered}
$$

Constraint (4.8) ensures that RTP can be serviced only by an active base station at an active site. By constraint (4.9) RTP can be serviced only by one base station at most.

\section{TRAFFIC COST}

Traditionally in wireless network planning problem the financial component of an objective function targets the minimization of the total expenses imposed by the infrastructure deployment costs, costs of equipment and maintenance. However, in this thesis we propose another approach to the financial model replacing the minimization of the expenses by the minimization of the traffic cost or cost of service. This objective reflects a cost of providing a unit of service, which in our case is measured in Kbps. The low cost of service ensures that the service provider can gain more profit by providing service to consumers at a competitive tariff. In other words the optimization 
algorithm will aim to create cost-effective networks and generate maximum possible profit.

$$
E_{3}=1-\frac{t r a f \_c o s t \_m i n}{\text { traf_cost }}
$$

where traf_cost_min is a lower bound on the cost of service or a desired level by the service provider, traf_cost is the traffic cost of the current network plan.

$$
\text { traf_cost }=\frac{\sum_{i=0}^{|S|}\left(z_{i} \times\left(S_{i}^{C A P E X}+S_{i}^{O P E X}+\sum_{j=0}^{\left|B_{S_{i}}\right|} z_{i-j} \times B_{i-j}^{C A P E X}\right)\right)}{\sum_{i=0}^{|R|} R_{i}^{\text {serv }} \times R_{i}^{\tau}}
$$

The numerator in the expression 4.11 provides the total cost of the network, whereas the denominator calculates the total traffic of serviced users in the network. The main contributors which make the most significant impact on the overall expenditure are the site installation and antenna equipment costs, which are the capital expenditures, and the annual site maintenance costs - operational expenditure. It is clear that the fewer sites are in use and the more antennas are deployed at each BS the less the overall costs will be.

\subsubsection{Key performance indicators}

As in the rest of the industries, key performance indicators (KPIs) are a set of critical measures of the performance of a network. Such measures are commonly used to help engineers and providers to define and evaluate how successful the network plan is. Typical list of these indicators in the Telecom industry is quite wide including such categories as: subscribers, usage, revenue, coverage and spread, market share, incremental performance, operational efficiency, marketing, service performance and quality, financial, spectrum efficiency etc. Each category may also include tens of 
different KPIs. In our case the list was limited to a few of the indicators reflecting the main technical and financial measures of the network performance. The defined KPIs are as follows:

- Coverage level $(\%)$

- Service level $(\%)$

- Expenses $(€)$

- Year 1 profit $(€)$

- Traffic cost $(€ / \mathrm{Kbps})$

- Spectral efficiency (bps/Hz)

- Total capacity (Mbps)

Some of the KPIs have already been described in the previous section such as coverage, service, traffic cost and the overall expenses. The remaining indicators are as follows:

\section{SPECTRAL EFFICIENCY}

As spectrum is a scares resource, spectral efficiency is one of the main performance indicators which allows to evaluate effectiveness of any of the wireless technologies providing triple-play services. Spectral or bandwidth efficiency refers to the information rate that can be transmitted over a given bandwidth in a specific communication system. It is a measure of how efficiently a limited frequency spectrum is utilized by the physical layer protocol. It is measured in bit/s/Hz and is obtained by dividing the channel capacity by the channel bandwidth. WiMAX Forum provides the spectral efficiency figures for different deployment scenarios of Mobile WiMAX networks, e.x. using SIMO or MIMO antennas, varying between 1.0 and $2.0 \mathrm{bps} / \mathrm{HZ}$, [117]. 


\section{PROFIT}

Calculation of profit is simply expressed as the difference between the total generated revenue from all users and total expenditure necessary to supply service:

$$
\Pi=\sum_{j \in R} R_{j}^{s e r v} \times R_{j}^{r e v}-\sum_{i=0}^{|S|}\left(z_{i} \times\left(S_{i}^{C A P E X}+S_{i}^{O P E X}+\sum_{j=0}^{\left|B_{S_{i}}\right|} z_{i-j} \times B_{i-j}^{C A P E X}\right)(4 .\right.
$$

The aim is to maximize the profitability of the network having that each serviced user brings revenue, the installation costs (or CAPEX) are paid at the initial stage of deployment and operational costs (or OPEX) are considered in each year. We do not consider the growth of the network in terms of number of users and consequent increase in the number of base station required for serving the new users.

\subsubsection{Neighbourhood moves: generating new solutions}

The main problem-specific choice of the algorithm and efficiency of SA is influenced by the neighbourhood function definition [111]. Creating an effective neighbourhood structure is a crucial part of designing an efficient simulated annealing algorithm. The following investigations [50, 57] state that the choice of the neighbourhood serves to enforce the search topology - 'a neighbourhood structure which imposes a smooth topology where the local minima are shallow is preferred to a bumpy topology where there are many deep local minima'.

Another factor playing a significant role in the choice of neighbourhood function is the neighbourhood size. Although there are different views on the scale of the neighbourhood, [40] stating that small size is favourable and that very large neighbourhood is randomly sampling the search space and is unable to target specific areas, and [114] reporting completely the opposite that larger neighbourhoods perform better. With no theoretical proof available the main conclusion can be made that the neighbourhood requires reachability in a finite number of steps from one solution to any other solution. 
The neighbourhood structure we have employed consists of a selection of so-called 'moves' as follows:

Add/Remove site - the site to change its operational status is chosen at random. Therefore when the site is not active a random number of BS's is activated with default configurations. When the site is operational it is deactivated and the status is changed accordingly.

Add/Remove sector - at a randomly selected operational site the choice is made whether to install additional antenna with default configurations or remove one.

Move site - for a selected active site the list of all possible not operational sites is created. Thereafter, from the set of the closest sites one is chosen at random and all the base stations from the initial site are removed to the new one, retaining all configurations.

Reconfigure antenna - a randomly chosen active antenna is selected for modification and one or several configurations are altered.

Reconfigure site - a random operational site is selected and all the base stations in it are modified.

The procedures of the moves and their usage vary with the three optimization stages which are aiming to resolve specific problems within a stage and imposing different limitations on the utilization. We therefore reflect all the specifics of each move for each stage in sections 5.3 and 6.1 .3 .

\subsection{AFP formulation}

Generally, frequency planning schemes can be classified into the following categories: fixed channel allocation (FCA), dynamic channel allocation (DCA) and hybrid channel 
allocation (HCA). Each of these methods has advantages and disadvantages and are applied to different technologies e.g. GSM, Wi-Fi, MBWA, WiMAX and also different multiple access schemes e.g. TDMA, FDMA, CDMA, OFDM or OFDMA schemes. An advantage of OFDMA over OFDM-TDMA and OFDM-CDMA is the elimination of intra-cell interference, avoiding the CDMA multi-user detection problem. FCA is mainly used in GSM systems where a group of channels are assigned to each cell or cluster of cells and fixed during the network operation. In comparison to FCA, the channels in DCA are allocated not only in the space domain to different base stations but also adapt to the distribution of channels over time as well. Taking advantage of multi-user channels, traffic diversity and precise estimation of channel conditions between users and base stations DCA overcomes the problems listed before but at the price of higher computational complexity and increased signalling overhead.

Significant interest has been dedicated to DCA algorithms for different technologies and in particular for 802.16d and 802.16e standards [127, 20, 92, 98, 90] taking into account the characteristics of OFDMA in recent years. On the other hand a lack of attention is devoted to the initial network deployment where both the ACP and AFP problems are tackled simultaneously [69]. Therefore, we introduce a new approach for simultaneous resolution of the ACP and AFP problems during the optimization for WiMAX mobile networks. The staged optimization framework presented here is used to ease the ACP problem by decomposing the entire problem into a set of tasks which are simpler to solve. Another contribution is a channel allocation algorithm which is performed during the solving of the ACP problem. Here the main issue lies in the online calculation of the SINR which determines whether the user is served by a particular base station and the QoS that can be supported at this SINR level.

\section{Slot Allocation Problem}

The main objective of AFP is to allocate available slots to users in order to maximize the system throughput based on fading channel and traffic conditions. The network has been defined as an OFDMA system in section 3.1. We use the following notations to 
describe network measurements:

1. Rate matrices $R^{\tau}=\left[R_{i}^{\tau}\right]$ and $R^{\tau}\left(L_{j}\right)=\left[R_{i}^{\tau}\left(L_{x}\right)\right]$, which represents $R_{i}$ 's user demanded bit-rate and achievable bit-rate with interference on slot $L_{x}$

2. Interference matrix $I^{x}\left(R_{i}\right)=\left[I_{j-k}^{x}\left(R_{i}\right)\right]$, which represents $R_{i}$ 's user list of interfering BS sectors on slots $L_{x}$

3. Slot assignment notations defined in section $3.5 .4 \delta_{i}^{x}$ and $\delta_{j-k}^{x}$, indicating which slots $L_{x}$ are assigned to users $R_{i}$ and sectors $B_{j-k}$ at sites $S_{k}$

Following these notations the slot allocation problem with the goal of maximizing network throughput can be formulated as follows:

$$
\sum_{i \in R} \sum_{x \in L} \delta_{i}^{x} \times R_{i}^{\tau}\left(L_{x}\right) \longrightarrow M A X
$$

subject to:

$$
\begin{gathered}
\forall x, j, k: \sum_{i \in R} R_{i-j-k}^{s e r v} \times \delta_{i}^{x}=1 \\
\forall i: \sum_{x \in L} \delta_{i}^{x} \geq 1 \\
\forall i: \sum_{x \in L} \delta_{i}^{x} \times R_{i}^{\tau}\left(L_{x}\right) \geq R_{i}^{\tau}
\end{gathered}
$$

Constraint 4.14 states that each slot within a sector can be assigned to one user and one user only, constraint 4.15 shows that each served user must be allocated at least one slot, and the number of slots assigned to a user must be sufficient to satisfy the user's traffic demand, 4.16 . 
The goal of the slot allocation algorithm is to identify the set of slots to assign to each user in order to maximize the overall system throughput. In other words a slot assigned to user $R_{i}$ is optimal if:

$$
\forall i, j, x: R_{i}^{\tau}\left(L_{x}\right) \geq R_{j}^{\tau}\left(L_{x}\right)
$$

Therefore, the aim of the algorithm is to assigning a slot to a user having the best channel response:

$$
R_{i}^{\tau}\left(L_{x}\right)=\underset{j \in R}{\arg \max } R_{j}^{\tau}\left(L_{x}\right)
$$

\subsection{Problem Decomposition}

Large problems in different areas of research are often solved in phases as they are very complex. For example in logistics, designing a distribution network involves determining locations for warehouses, designing their internal systems, determining the composition and routing of the delivery fleet, scheduling the labour forces etc. Each is a complex decision in its own right, but all these decisions interact.

Although techniques based on meta-heuristics have proven to be successful for particular optimization problems, the real world large scale problems are too complex to be solved with these techniques, which makes them considerably computationally (and run-time) costly to produce good quality results. Instead, specialized and problemspecific algorithms tend to perform best. An effective approach to this problem is application of decomposition techniques.

Definition 4.3.1. Decomposition: the break down of a complex problem into smaller parts and solving each of them separately, either in parallel or sequentially (in phases). 
The advantages of problem decomposition are:

- it facilitates the understanding of the trade-offs of the system.

- it simplifies modelling as subproblems are relatively simple, which helps the engineer focus on a small set of clearly defined issues.

- it makes the adaptation to changing conditions of the problem easy to implement as the purpose of a model is always clear.

- it is a good alternative to the construction of a complex and detailed model which incorporates all issues in a single model.

A standard approach would entail the creation of one large-scale model which will incorporate all the information and relationships relevant to the network design issues. Apart from the question of whether it is feasible to create such a model one can seriously doubt the contribution such a model would make to the quality of the decision making process. Because the search space associated with such a model would necessarily be very large it would be difficult to understand the results obtained since the underlying mechanisms are too complex to comprehend. Moreover, such a model would have very heavy computational requirements.

There are two types of decomposition - one which works in one step called separable. The problem is partitioned into subproblems and then each of these subproblems is solved separately (and in parallel). The solution is then re-assembled from the results of parts. Second type - when there is some interaction between the subproblems, the problem cannot be solved independently. The overall problem in this case is solved by iteratively solving a sequence of smaller problems, where the solution of one stage becomes the input for the following stage.

This section proposes a problem decomposition approach to solve ACP and AFP problems. The aim of this scheme is to break the complex problem into three easier to manage sub-problems and solve them sequentially in order to improve the algorithm's 
result in terms of both solution quality and runtime. This method does not guarantee finding the absolute optimal solution, however proves to be more effective in finding better quality solutions for the large scale network instances and to reduce the processing time. Moreover, we are interested in the trade-off between solution quality and runtime rather than finding the global optimum. This is more practical approach when the time available to produce solution is constrained.

\subsubsection{Background}

Decomposition in optimization is not a new idea which has been widely applied in different applications of computer science. A good reference on decomposition methods can be found in [34]. This reference provides a good insight on the decomposition methods such as primal decomposition and dual decomposition. As well as explore general decomposition structures and associated decomposition methods.

With application to wireless network planning, [43] proposes a problem decomposition approach to solve hard Frequency Assignment Problem instances with standard meta-heuristics. The results show that the use of the decomposed assignment approach proves to be a very effective technique to solve large practical data sets using meta-heuristics when time is limited. Furthermore, they show that the decomposition approach allows the use of standard meta-heuristics irrespective of the scale of problems it is applied to, hence eliminating the need of tailoring specifically designed algorithms for a particular class of problems. Another approach in the area of Automated Cell Planning has been applied in [23] using the DesNet system which aims to solve the problem of planning cellular systems using Frequency-Division Time-DivisionMultiple-Access (FD-TDMA) technology. Their framework adopts an approach based on simulated annealing. A three phase approach is proposed to automate the design process. Their experimental results show that the phased approach proves to be far more effective than an un-phased alternative. Similar work is presented in [116] with the aim of enhancing the WCDMA network designing process. 


\subsubsection{Decomposition approach}

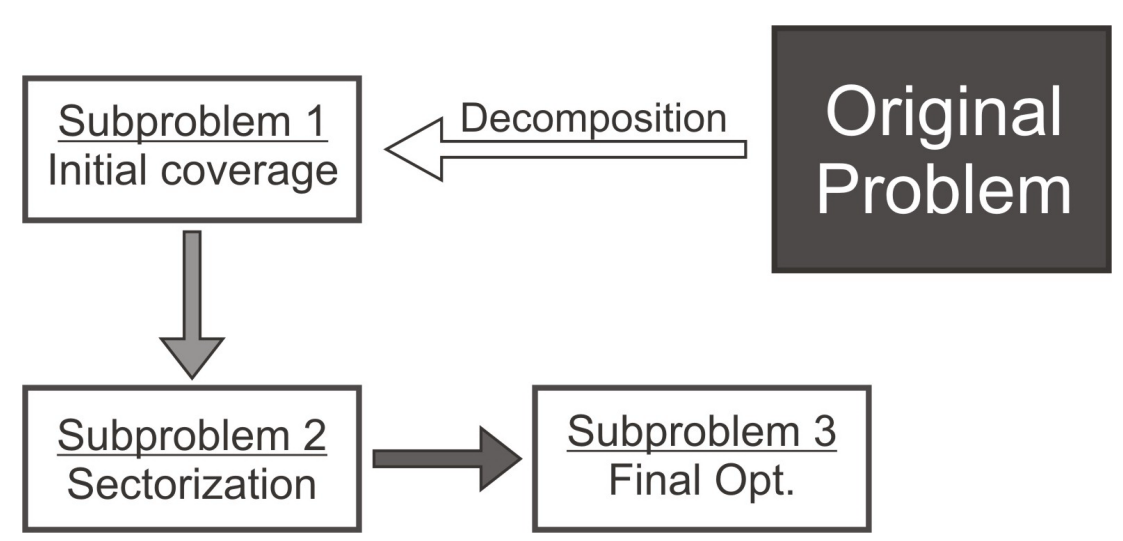

Figure 4.2: Decomposition of an overall problem into sub-problems

The framework proposed in this thesis is aimed at simplifying a complex problem of WiMAX network planning by dividing the optimization process into three stages, instead of increasing the complexity of a meta-heuristic which solves the problem as a whole (see Fig. 4.2). Each stage is dedicated to achieving a specific set of goals in order to simplify the overall optimization procedure and gain better results compared to a single stage optimization solving the complete problem. The three stages are as follows:

Stage 1 overview - aims to generate an initial cell plan with maximum coverage and service levels while minimizing the overall financial commitment for the required return period. For this reason, only three objectives are considered in this stage, which are: service level $\left(E_{2}\right)$, cost $\left(E_{3}\right)$, and traffic cost $\left(E_{3}\right)$. The optimization objective is achieved through the thorough selection of sites for deployment of base stations and their configuration. All the antennas deployed at this stage are omni-directional with artificial capacities simulating a number of sectors with directional antennas. Consequently, the resulting output of this stage provides:

- a set of site locations to be activated,

- a set of regions (set of RTPs) in the network that are "covered" by each site, 
- a number of sectors required for each site to provide service for the given areas.

Stage 2 overview - aims to sectorize the initial cell plan by replacing synthesized omnidirectional base stations with the number of real sector antennas that was predicted in the initial coverage stage, and performing preliminary configuration so that the given regions are covered and any global constraints are met. All the changes are individually made for each site in the order from the largest cell to the smallest. The objectives to be improved in this stage are: service $\left(E_{2}\right)$ and assignment ratio $\left(E_{4}\right)$ (Section ??). It is conducted reasonably quickly for the stage to obtain the resulting cell plan.

This procedure is individually performed for each site and involves following steps:

1. Evaluate the users and traffic demand distribution relative to a given site position. This is to calculate a centre of mass for the site's coverage region and hence plan the sectors where the base stations are more likely to be installed for a high coverage level and for the most efficient traffic load.

2. Taking into account all calculations and information derived from the previous step, antennas are reconfigured in an intelligent way, but in a random order, while considering given objectives and constraints. This is continued for a number of iterations.

In this stage the AFP algorithm is performed by allocating slots to a user assigned to a base station, together with the evaluation of interference in these slots. This determines the modulation scheme for transmission and achievable bit-rate at the SINR level.

Stage 3 overview - takes into account the full network for optimization where the complete set of neighbourhood moves is used in order to target any deficiencies in the design from stage 2 , the main objective is to increase the expected profitability while ensuring all constraints remain satisfied.

To summarize, the main goal of the 3 stage optimization framework is to: 
1. identify a subset of site locations

2. deploy sectorized base stations

3. configure each sector

It can be noted that the initial stage involving the exploration of sites to be activated plays a crucial role for the service provider as the results of this phase of network planning define the future potential of the network in terms of profitability and possibility of expansion and growth. The developed decomposition strategy allows us to overcome complexities associated with the overall problem. Full details of Stage 1,2 and 3 will be presented in the following chapters. 
Chapter 5

\section{Optimization and Dimensioning -}

\section{Stage 1}

Stage 1 is designed to partition the entire network into cells with maximum coverage and service levels while minimizing the total expenditure. The idea is to execute this stage in the shortest amount of time to get an initial perspective on the best design. This includes estimation of the required number of sites, determination of the best possible site locations which optimally cover the network area, and partitioning the network into cells that can be serviced from the obtained sites. In [97] several schemes were developed for building the initial network plan. Although different types of regions are taken into consideration with low and high densities of population representing rural and urban areas the schemes are limited to the usage of 3 sectored base stations (120 degrees separation) or omni directional antennas. This approach inevitably leads to an excessive usage of sites, which significantly increases the total expenditure and degrades future financial return. Moreover, the poor quality of the initial design will eventually drive the subsequent optimization phases to struggle to converge to an efficient and profitable final network solution. To overcome this problem instead of sectorizing the base stations from the start and using directional antennas we reduce the complexity of the problem by utilising a synthesized representation of omni directional antennas which simulate a number of sectors with directional antennas but with an omni radiation pattern. This significantly reduces the amount of configurations and decision variables to perform optimization for the stage, consequently improving the 
chances of finding a good quality solution and reducing the runtime. At the same time it artificially raises the capacity of a single base station. In addition, the combined use of path-loss figures from the active sites and omni radiation antenna patterns produce cells with near optimal sizes and shapes which benefits the following optimization stages. These representations of the real sectored base stations lead to the following assumptions:

Assumption 5.0.1. The capacity of each omni-directional antenna is not fixed to a single capacity, but has a variable value which is limited by the minimum $N_{\text {min }}^{\text {sect }}$ and

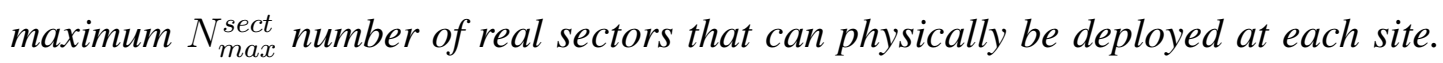
The restrictions are due to spectrum limitations or limited number of channels. This capacity can be set in two ways, either when it is selected to optimally service the area surrounding the site or when it is forcefully increased or decreased. This process is described in section 5.2

Assumption 5.0.2. Though it is not typical for omni-directional antennas to be tilted, in our case the pattern can be artificially amended (explained in the following section $5.3)$.

Assumption 5.0.3. Compared to a sectorized base station where each sector can perform transmission at a selected/predefined power level, for the omni-directional antenna the radiated power is the same for all directions in a 360 degrees horizontal plane.

The initial stage is outlined in Figure 5.1 which involves the following process. First, the candidate site is chosen from a random ordering. If the site is active then according to the selection scheme the next move is chosen to perform some small or large changes to the current BS configurations. Alternatively if the site is inactive then it is activated with a base station configured to default settings and with optimally chosen capacity. After the move has been executed the obtained solution is evaluated. Whether accepted or not, this state is considered to update the 'acceptance ratio' of each move. This in turn determines the selection probabilities of moves (described in Section 4.1.4). 


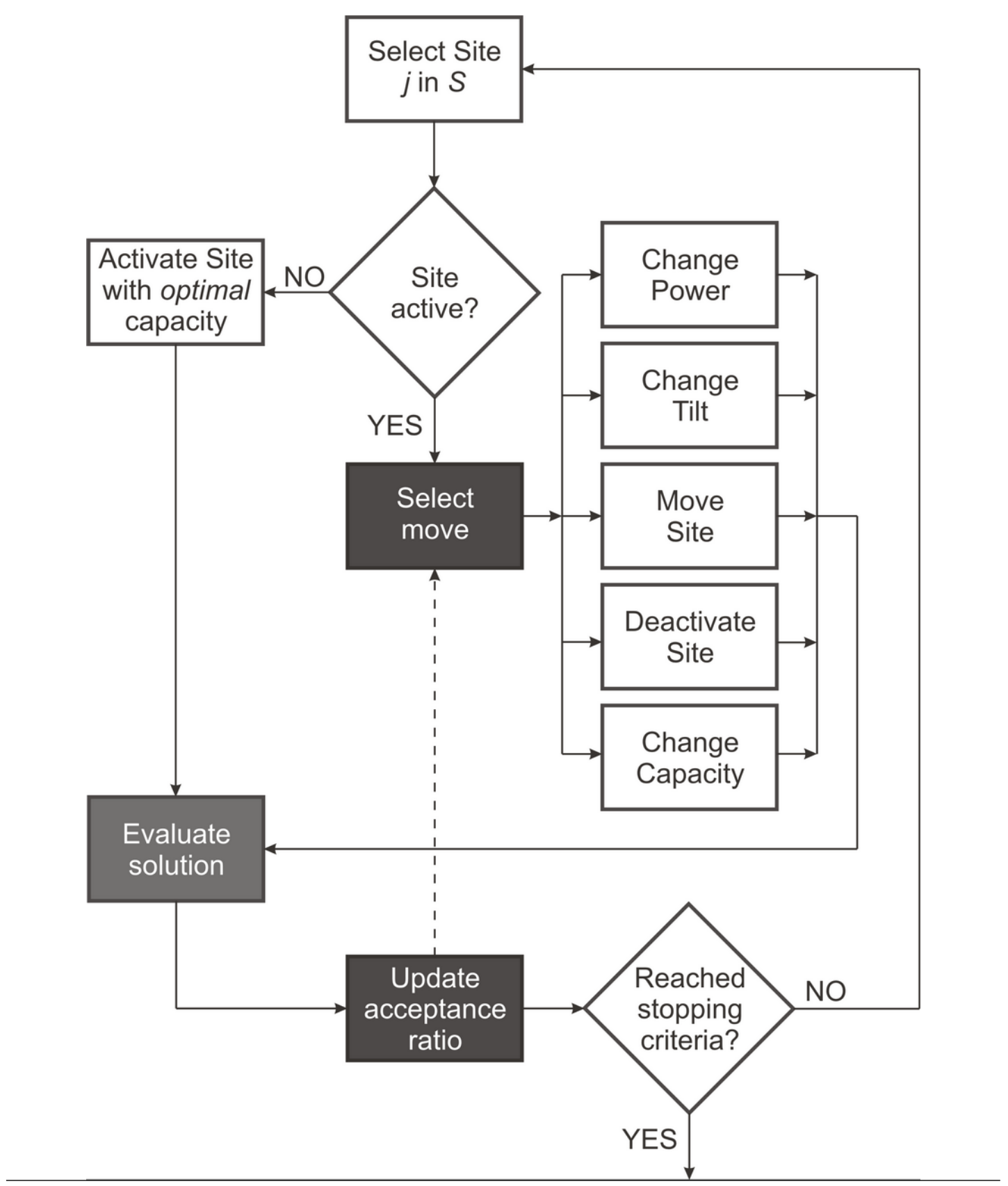

Figure 5.1: Initial coverage stage scheme

Figure 5.2 illustrates the main issues related to the use of antennas in the following sections. 


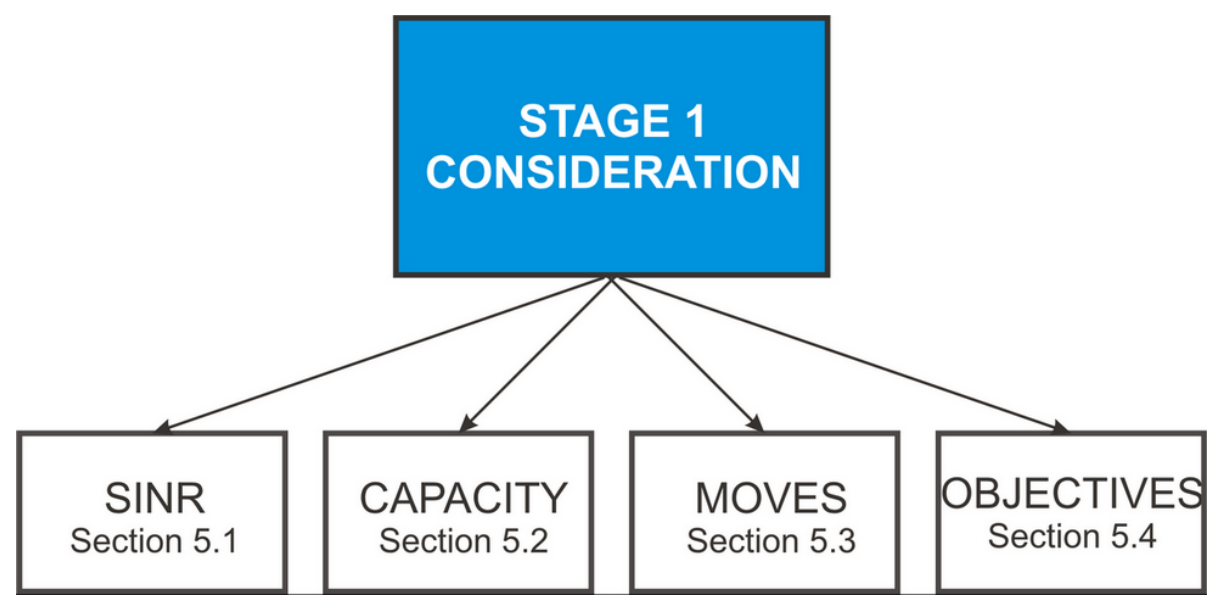

Figure 5.2: Main issues related to Stage 1 optimization

\subsection{Interference and throughput calculation}

There are several issues which have to be addressed in this part. First, as each BS simulates a hypothetical number of sectors with directional antennas realistically we cannot estimate the real SINR for the serviced users. This is due to the fact that without the sectors being deployed the slots cannot be allocated to users without knowledge of the sector they are allocated from. Second, as the interference level is not known the modulation scheme and hence the achievable bit-rate which can be supported at a particular SINR cannot be determined, as well as the required number of slots to satisfy the users traffic demand. To solve these problems we propose a new framework based on simple argumentative assumptions to identify the SINR for each served user.

The idea lies in the determination of overlap between the base stations - the more base stations that cover a user the higher level of interference they create. If the assignment of frequency resources is not performed in Stage 1, which means the actual SINR cannot be evaluated, then we overcome this by introducing an approximation representing the relation between the level of overlap and the attenuation of the serving signal due to the potential interference. The procedure is as follows.

Having deployed a BS, first a set of covered users is identified, followed by the estima- 
tion if these users can be serviced or not. In order to calculate this, the algorithm loops through all the BS covering the current user. With each potentially interfering site we predict the number of sectors which will be covering the user by looking at its capacity (or in other words at the number of sectors). However, obtaining the total number of sectors at a neighbouring site is not the final solution to a problem. It can be noted that the base station with 6 sectors will most likely have more sectors interfering with a given user in comparison to a base station with only 2 sectors (Figure 5.3). Consequently, this measure needs to be interpreted into a proportion of sectors (after the omni base station has been sectorized) which will actually be covering the current user. To assess this we have plotted the following graphs through the series of experiments, Figure 5.4

The average amount of interfering sectors versus the distance between the user and the corresponding site for $n$-sectorized base stations (with 6, 5, 4 and 3 sectors) can be seen on these graphs. An interesting observation is that approximately beyond 500 meters from the interfering site the number of interfering sectors stabilizes and behaves steadily over the distance, whereas in the closer proximity to the user the number of sectors dips slightly following the sharp increase the closer it gets to the site.

A reference table in Table 5.1 has been formed to reflect the relation between the capacity of an omni base station and the number of interfering sectors.

\begin{tabular}{|c|c|c|}
\hline \multirow{2}{*}{ BS capacity } & \multicolumn{2}{|c|}{ Interfering sectors } \\
\cline { 2 - 3 } & $>500 \mathrm{~m}$ & $<500 \mathrm{~m}$ \\
\hline 6 & 2.3 & 3 \\
5 & 2 & 2.5 \\
4 & 1.7 & 2.2 \\
3 & 1.3 & 1.8 \\
2 & 1 & 1 \\
\hline
\end{tabular}

Table 5.1: Number of interfering sectors as a function of capacity of omni BS and distance between user and interfering site. 

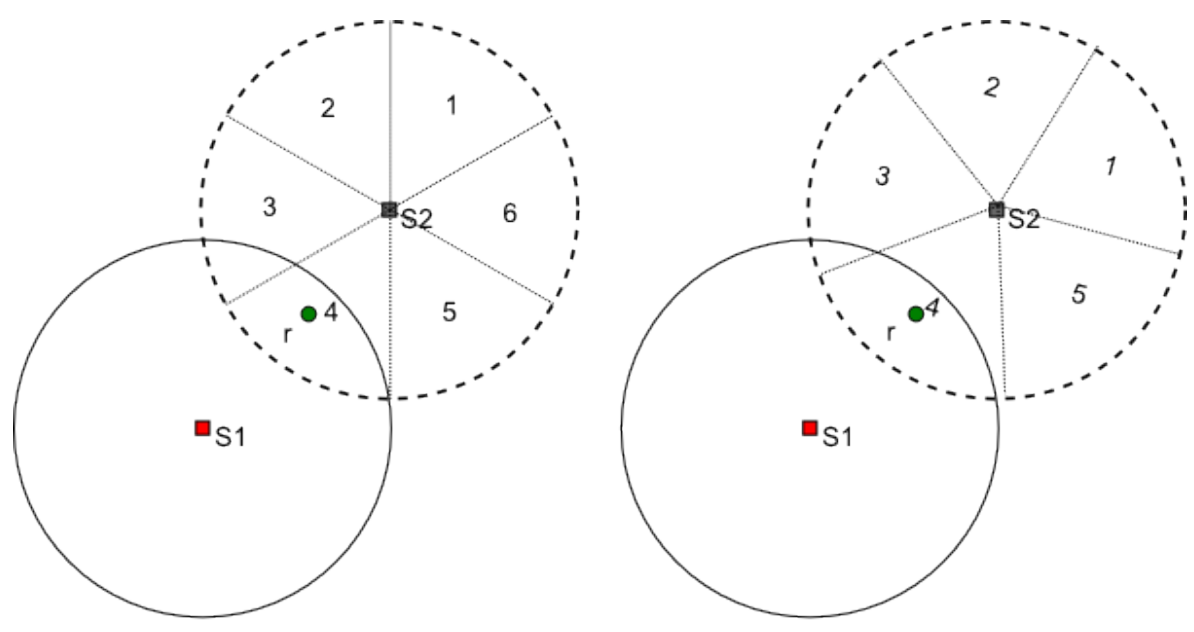

6 sectored base station

5 sectored base station

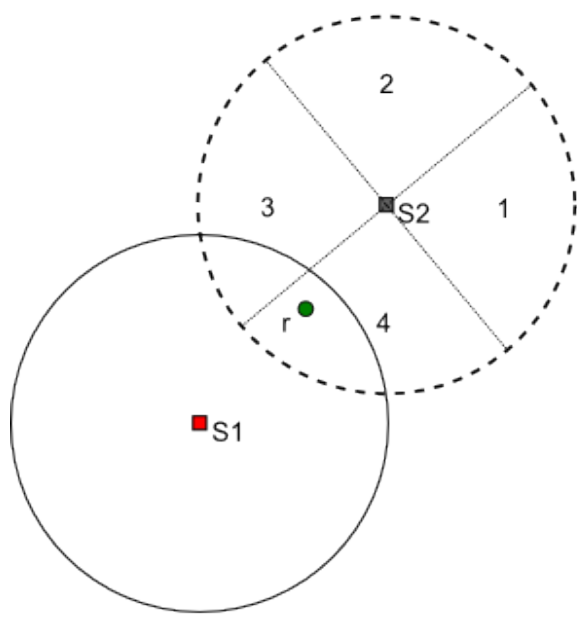

4 sectored base station

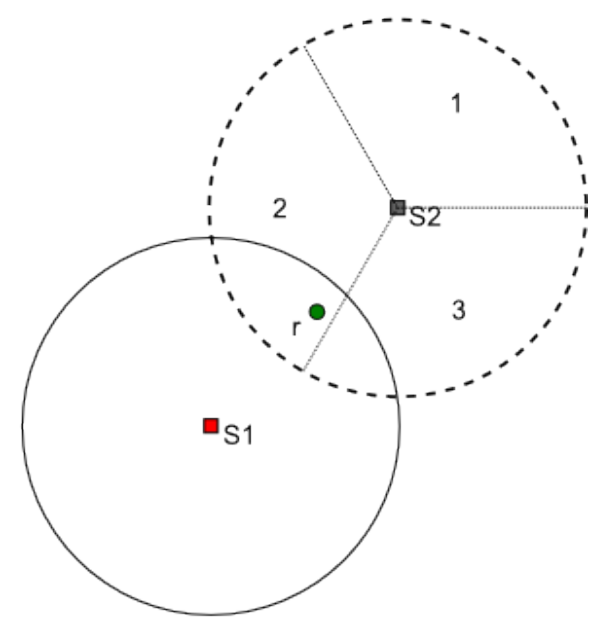

3 sectored base station

Figure 5.3: Sectorization of omni-directional base stations - evaluation of the interfering sectors. Square with red mark represents the serving base station, with black square in the centre - interfering base station, and green circle - a user. 

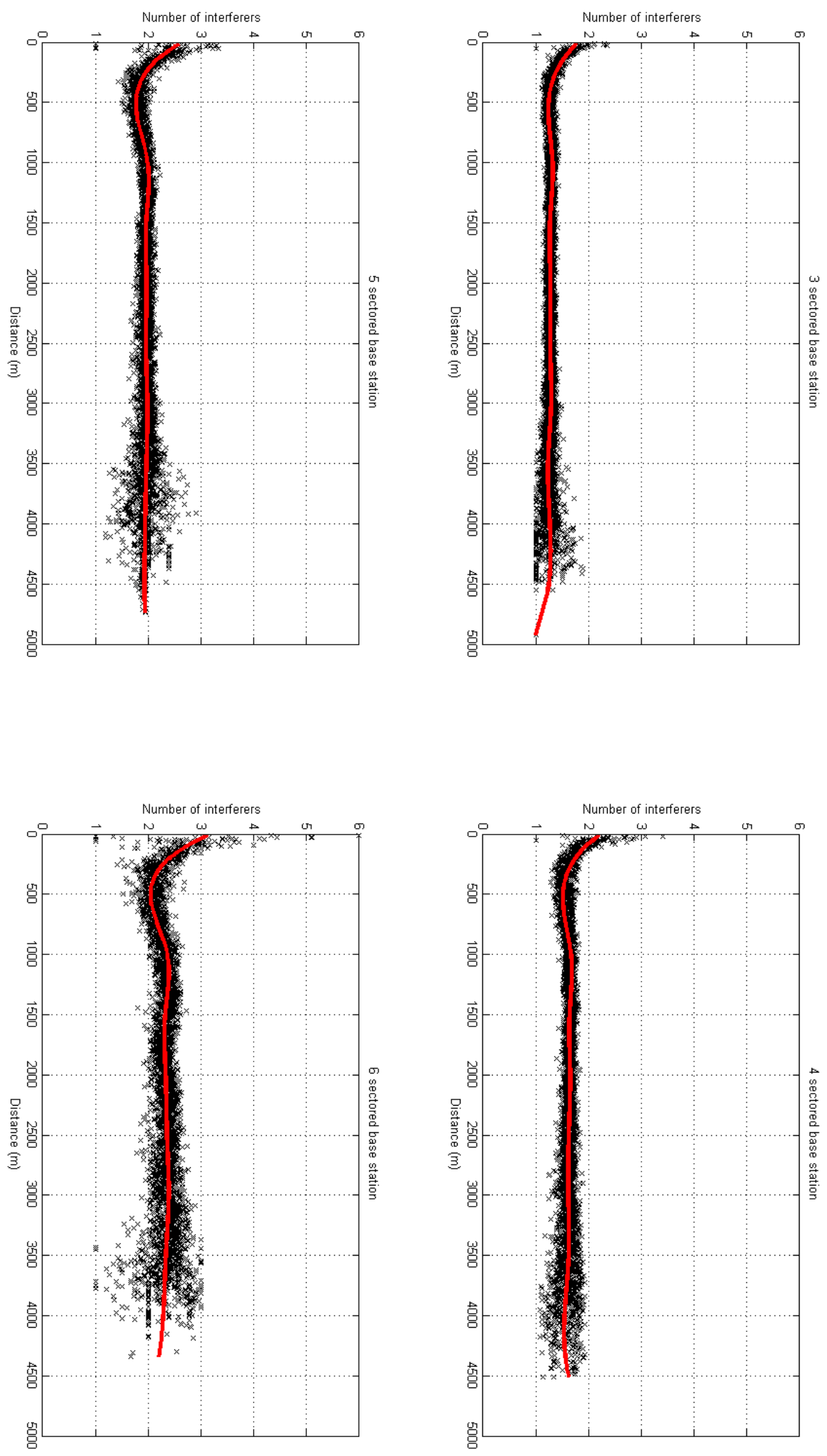

Figure 5.4: Identification of interfering sectors at omni-directional base stations 
Despite the growth trend of interfering sectors within the 500 meters distance we average the value for this interval to simplify the calculations.

Having identified the relation between the capacity of an interfering base station and the number of sectors the next step is to define an expression for the SINR calculation. Similar to expression 3.4 we introduce a modified version for Stage 1 operation:

$$
\begin{gathered}
\gamma_{i}=\frac{F_{b-s}\left(R_{i}\right)}{\sum_{S_{k} \in S} \sum_{B_{j} \in B_{S_{k}}} I_{j-k}^{o m n}\left(R_{i}\right)+\Gamma_{i}} \\
I_{j-k}^{o m n}\left(R_{i}\right)=F_{j-k}\left(R_{i}\right) \times n_{i n t}\left(B_{j-k}^{c a p}, d_{i-k}\right) \times \epsilon_{c o l}
\end{gathered}
$$

where $I_{j-k}^{o m n}\left(R_{i}\right)$ is the interfering power experienced by user $R_{i}$ from the base station $B_{j-k}$ at the site $S_{k}, n_{i n t}\left(B_{j-k}^{c a p}, d_{i-k}\right)$ gives the number of interfering sectors presented as a function of BS capacity and distance between user $R_{i}$ and site $S_{k}$, (obtained from table 5.1) and $\epsilon_{\text {col }}$ is the average ratio of collisions in a single slot. Following the methodology of forming subchannels in Section 3.5.3 it has been identified that with all the permutation possibilities collisions between subchannels range between 0 to 24 (when subchannel, symbol and PermBase completely match) subcarriers, the average amount nevertheless is 2 subcarrier collisions per slot. With these assumptions we can finally calculate the SINR level for omni directional antennas using expressions 5.1 and 5.2. To summarize the overall procedure for the SINR estimation can be seen in Algorithm 5.1 


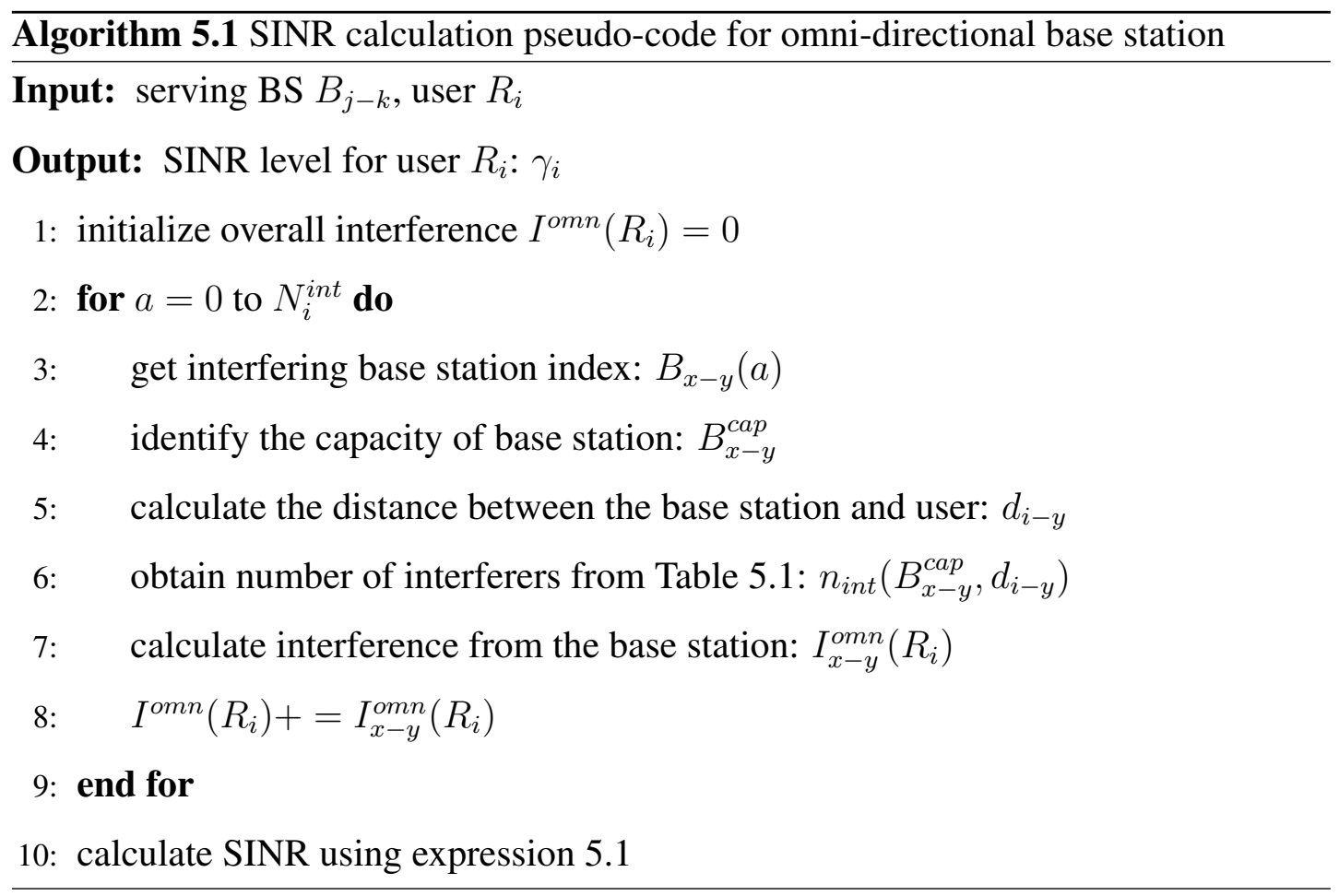

\subsection{Capacity estimation for omni-directional base sta- tions}

An accurate estimation of optimal capacity is not an easy task. Once again, the capacity of a base station with an omni-directional antenna in Stage 1 of the optimization framework defines the number of real sectors for the subsequent re-configuration of the base station in later stages. As it determines the quantity of available time/frequency resources (slots), the capacity adjustment makes a considerable impact on the size and shape of a cell. This reproduction of the omni cell needs close correlation to the physical behaviour of the real sectorized base station, in order to successfully design the overall network instance.

There are two ways in which the capacity can be set for a base station: fixed, or in other words the algorithm chooses the capacity with some predefined selection mechanism and fixes it for the base station regardless of the capability of a base station to serve the 
surrounding users; and optimal, where the algorithm evaluates the network, considers the neighbouring stations, the interference they create, the number of users the base station can possibly handle and the overall gain in terms financial return for choosing a particular capacity level. The fixed mechanism can be easily implemented and discussed in Section 5.3. The optimal selection procedure is discussed in this section and detailed in Algorithms 5.2 and 5.3 . It can be presented as the following sequence of actions:

- a site is selected for a base station activation;

- the list of all users covered by the BS is determined and ordered in descending order of received signal strength, strongest first;

- if the user is not currently assigned to any of the base stations the required number of slots to satisfy users traffic demand is calculated as a function of SINR;

- if the user is assigned to one of the base stations, but not to the selected one and the strength of the signal from the serving base station is weaker, then the number of required slots is determined in order to serve the traffic demand;

- if the user is already serviced by the selected base stations the number of previously assigned slots is obtained;

- after all covered users are analysed all the slots are summed up;

- the total number of slots is then divided by the number of slots in a single frame which gives the capacity of a current BS in terms of the amount of sectors.

\subsection{Operational moves}

At each iteration of the optimization process a site for the next operational modification is selected according to the move selection mechanism. The following moves have been selected for Stage 1: 


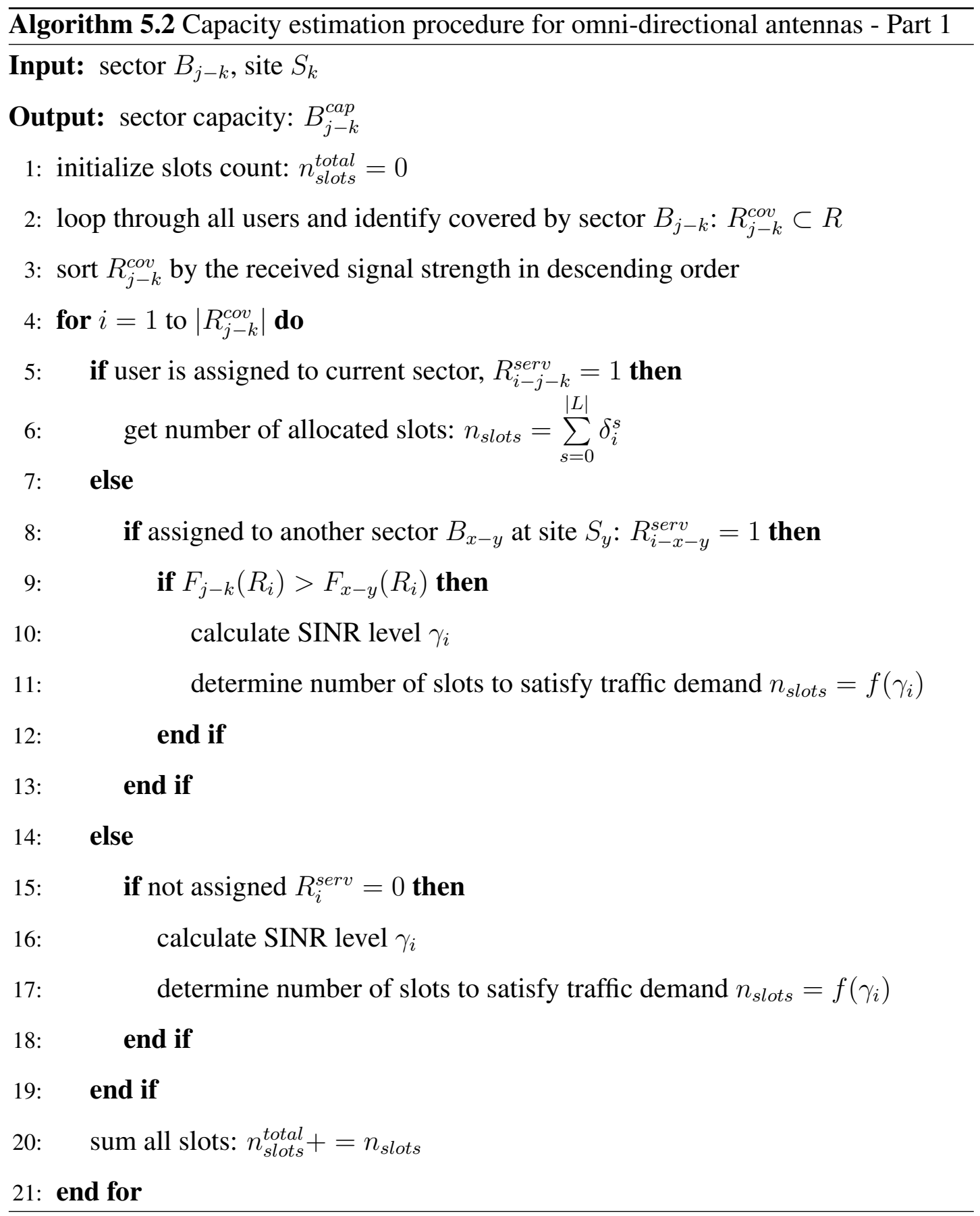

I. Site activation - if the site is not active its operational status is changed and a base station is deployed with default configurations (Table 5.2). The chosen capacity is 'optimal' (section 5.2) with regard to the configurations of the base station and its capability to serve the surrounding area. The procedure of activating a base station is reflected in Algorithm 5.4 . 


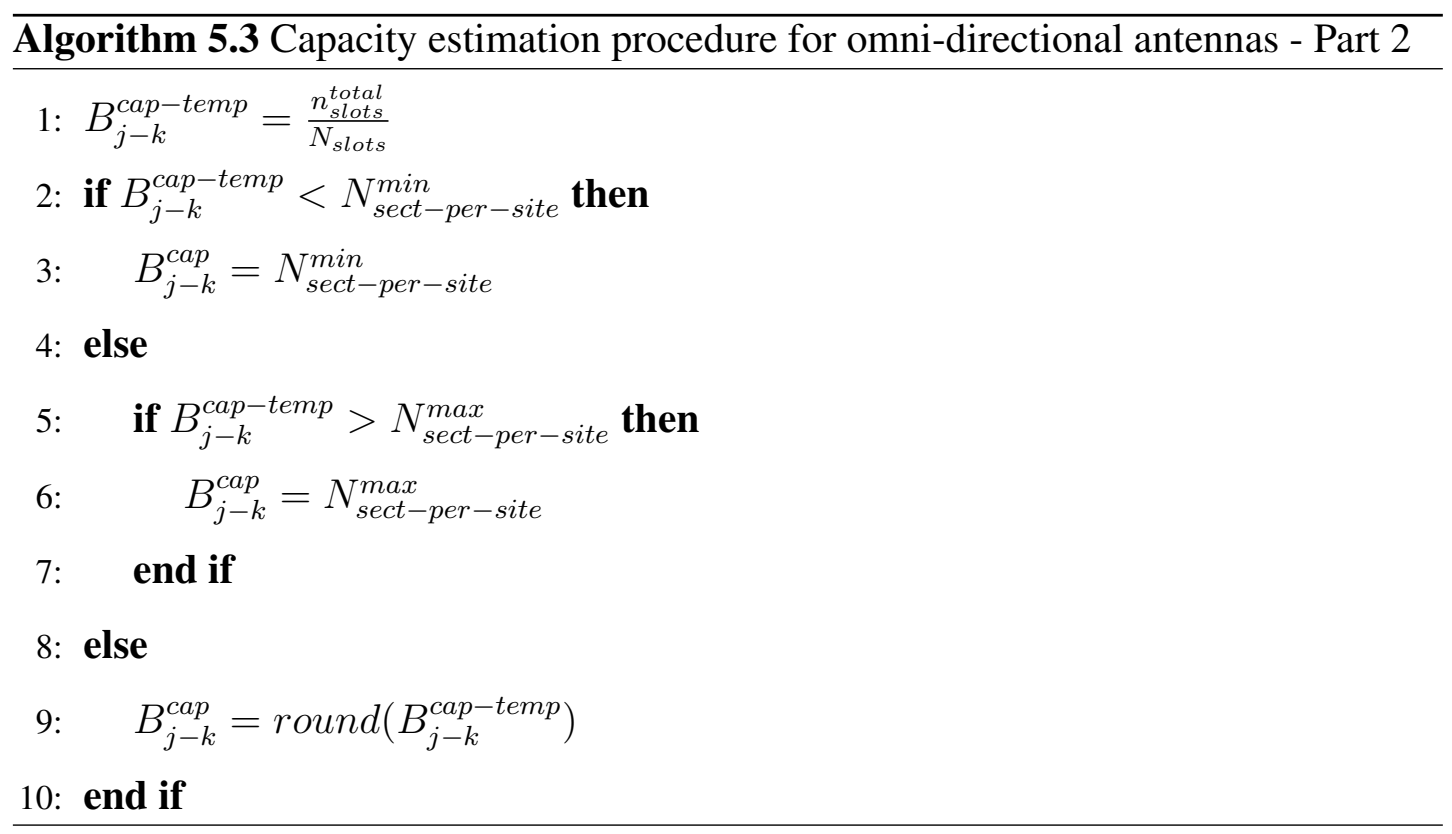

\begin{tabular}{|l|l|}
\hline Parameter & Value \\
\hline slot power & $36 \mathrm{dBm}$ \\
tilt & 11 degrees \\
capacity & 6 sectors \\
\hline
\end{tabular}

Table 5.2: Default settings for omni-directional base station

The aim of the algorithm is not only to identify the set of served users but also to evaluate the impact on neighbouring cells, the amount of interference it will produce and how it will affect the performance of other base stations. If a user is assigned to another base station $B_{x-y}$ and the current BS $B_{j-k}$ creates a better SINR level, and it holds enough slots for service, the user is re-assigned and the affected BS $B_{x-y}$ is added to the set $B^{\text {eval }}$ of cells for further re-evaluation to improve the loading of the base stations. On the other hand, if the current base station cannot offer better signal conditions the users' SINR level is re-calculated due to the introduced interference (described in Section 5.1). If the new SINR requires more slots and the base station is not able to offer this amount the user is unassigned. The same action is taken when the cell is fully loaded, and not able to assign any more users.

After the assignment of users is completed the set of affected cells, $B^{\text {eval }}$, is retrieved 
$\overline{\text { Algorithm 5.4 Omni-directional base station activation procedure }}$
Input: sector $B_{j-k}$, site $S_{k}$

Output: list of covered and served users: $R_{j-k}^{\text {cov }}, R_{j-k}^{\text {serv }}$

1: get list of covered users sorted in decreasing order of signal strength $R_{j-k}^{c o v} \in R$

2: identify optimal capacity for the base station $B_{j-k}^{c a p}$

3: initialize number of used slots at base station $B_{j-k}^{s l-u s e d}=0$

4: for $i=1$ to $\left|R_{j-k}^{c o v}\right|$ do

5: $\quad$ calculate SNR level $\gamma_{i}^{j-k}$

6: calculate amount of slots required for service of user $R_{i}: L\left(R_{i}\right)=\sum_{s \in L} \delta_{i}^{s}$ : $\sum_{s \in L} \delta_{i}^{s} \times R_{i}^{\tau}\left(L_{s}\right) \geq R_{i}^{\tau}$

7: $\quad$ if $\left|L\left(R_{i}\right)\right| \leq\left(B_{j-k}^{c a p}-B_{j-k}^{s l-u s e d}\right)$ then

8: $\quad$ if $R_{i-x-y}^{\text {serv }}=1$ : user is assigned to a base station then

9: $\quad$ get SNR level of serving station $\gamma_{i}^{x-y}$

10: $\quad$ if $\gamma_{i}^{j-k}>\gamma_{i}^{x-y}$ then

11: $\quad$ re-assign user $R_{i-x-y}^{\text {serv }}=0 \rightarrow R_{i-j-k}^{\text {serv }}=1$

12: $\quad$ include sector $B_{x-y}$ to the list $B^{\text {eval }}$ for re-evaluation

13: $\quad$ else

14: $\quad$ re-calculate $\gamma_{i}^{x-y}$

15: $\quad$ re-calculate required number of slots $n_{\text {slots }}=f\left(\gamma_{i}^{x-y}\right)$

16: $\quad$ if $n_{\text {slots }}<\left(B_{x-y}^{c a p}-B_{x-y}^{s l-u s e d}\right)$ then

17: $\quad$ unassign user $R_{i-x-y}^{\text {serv }}=0, R_{i}^{\text {serv }}=0$

18: $\quad$ end if

19: $\quad$ include sector $B_{x-y}$ to the list $B^{\text {eval }}$ for re-evaluation

20: $\quad$ end if

21: $\quad$ else

22: $\quad$ assign user to base station $R_{i-j-k}^{\text {serv }}=1, R_{i}^{\text {serv }}=1$

23: $\quad$ end if

24: else

25: $\quad$ if $R_{i-x-y}^{s e r v}=1$ then

26: re-calculate $\gamma_{i}^{x-y}$

27: $\quad$ include sector $B_{x-y}$ to the list $B^{\text {eval }}$ for re-evaluation

28: $\quad$ end if

29: $\quad$ end if 
for processing. If an affected cell has lost users due to the activation of another and gained available slots, the algorithm makes a quick loop through the covered users once again and checks the users which are not assigned to any of the cells. Provided that the users' SINR level requires less slots than BS has available, the user gets assigned and the affected base station recovers in terms of loading.

II. Site deactivation - when a selected site is active and is selected for deactivation, the BS is removed along with all the associations with the covered and serviced users. The released users are then re-evaluated in terms of being allocated to the neighbouring base stations. The neighbours are sorted in descending order of signal strength. The stations with the strongest signal level (above receiver sensitivity) and with the required number of slots available to serve the user is chosen. The user is then re-allocated to the best server. Figures 5.5 and 5.6 show the effect of a base station being deactivated. As one of the interferers has disappeared the overall SINR level in the network has improved, hence some of the remaining base stations have gained extra available slots and assigned additional users, Table 5.3 .

III. Change power - when this move is selected there are several options in the way the power level can be changed - small (MIN) or larger changes (MAX), which are both beneficial when performing search through the solution space. The pseudo-code is presented in Algorithm 5.5. First, the old base station configurations are saved. Then the mode is randomly selected between MIN and MAX. In MIN mode it randomly changes the power level by one unit (from the list 3.6). Alternatively, MAX mode selects randomly from the full power range excluding the old setting. After the new power level is obtained the base station is deactivated and re-activated with the new power setting.

IV. Change tilt - as was mentioned before it is not possible in the real world to change the tilt of omni directional antenna. Ideally omni antennas radiate power equally in all directions in the horizontal plane and with fading gain towards the south pole in the vertical plane, eliminating any need to tilt it. However, as we assume in Stage 1 


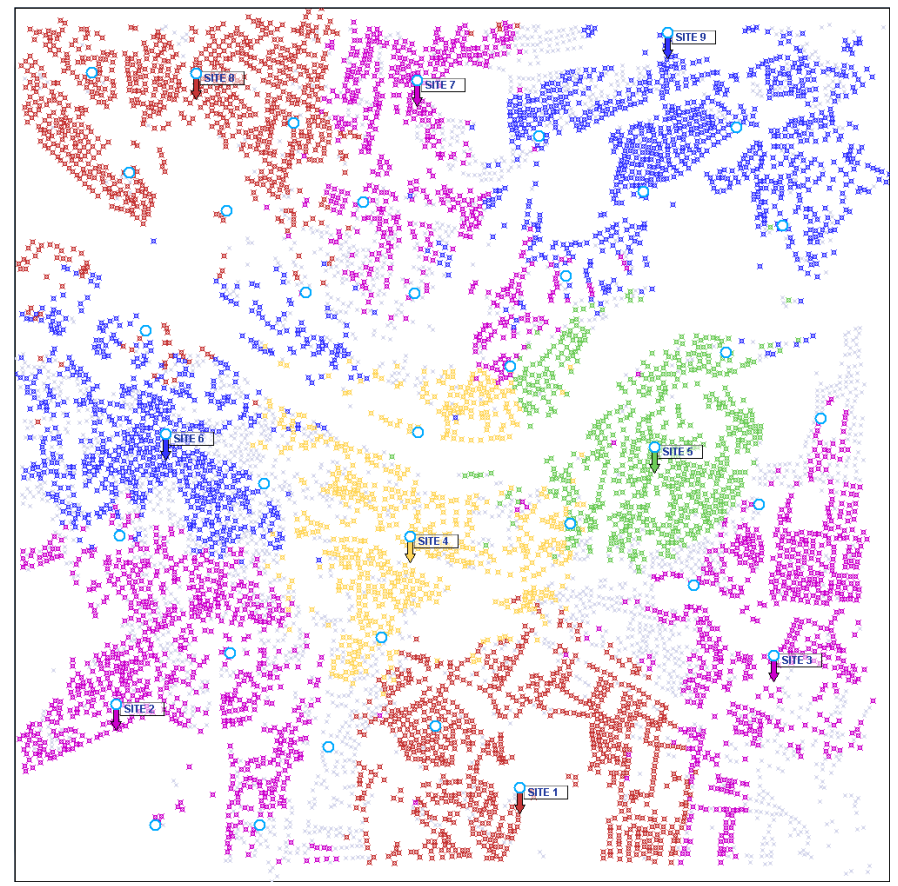

Figure 5.5: Original network before deactivating base station

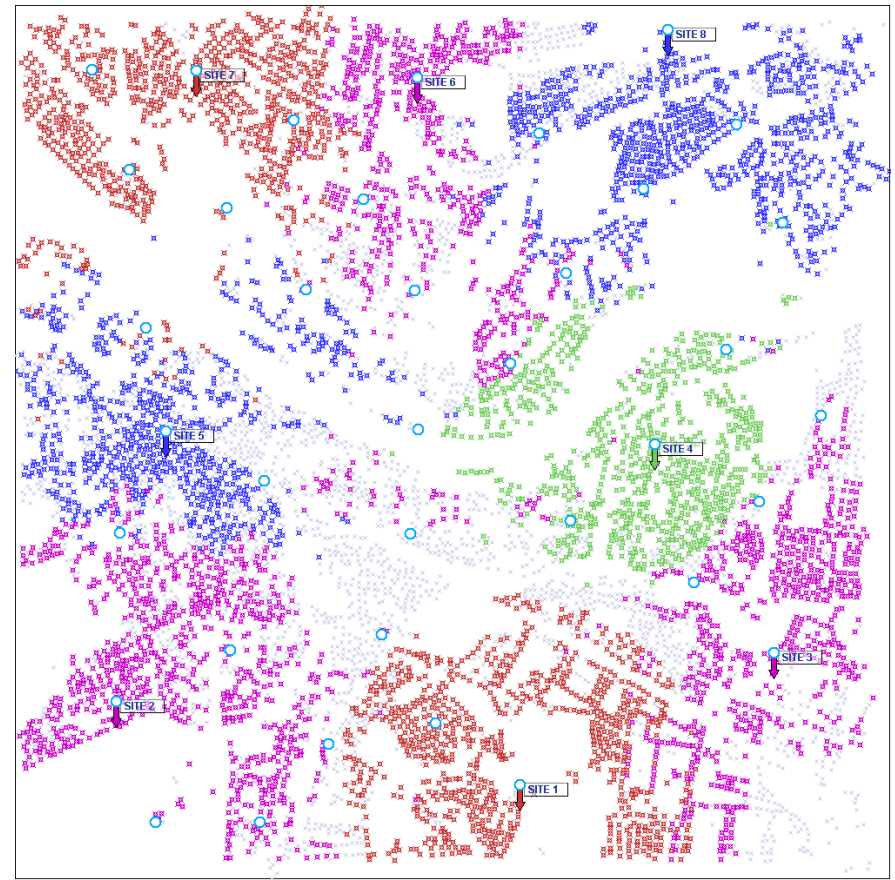

Figure 5.6: Network after deactivating base station 


\begin{tabular}{|c|c|c|c|c|}
\hline Sites & Site ID & Capacity & Serviced users before & Serviced users after \\
\hline 1 & 2 & 6 & 754 & 812 \\
2 & 5 & 6 & 713 & 784 \\
3 & 6 & 6 & 638 & 680 \\
4 & 10 & 6 & 626 & 0 \\
5 & 15 & 6 & 622 & 766 \\
6 & 16 & 6 & 687 & 716 \\
7 & 33 & 6 & 505 & 591 \\
8 & 34 & 6 & 826 & 815 \\
9 & 36 & 6 & 848 & 826 \\
\hline
\end{tabular}

Table 5.3: Variation in the number of serviced users before and after deactivation of site in Stage 1.

that an omni antenna represents a number of directional antennas which in turn can be adjusted with a tilt angle we introduce this feature as well. Changing the angle of the main lobe presents the opportunity to more effectively identify the optimal cells required for the subsequent stage $2 \& 3$. The idea behind it is simple - tilting $\alpha$ degrees translates into shifting the gains in the vertical pattern by this value. For example, the default pattern will be associated with 0 degrees tilt, but if we tilt it by $\alpha$ degrees then in order to calculate vertical loss of antenna pattern between the user and the site we will need the following angle:

$$
\theta=\sigma-\alpha
$$

where $\sigma$ is the angle between the RTP and the site. The idea is adopted from the electrical tilting of antennas when the pattern is not amended physically but computationally.

The procedure for changing the tilt is similar to the change power move with the exception that the set of available tilts is set to 15 values - between 0 and 14. The example of tilting an omni-directional pattern can be observed in the following figures: 5.7 and 5.8 


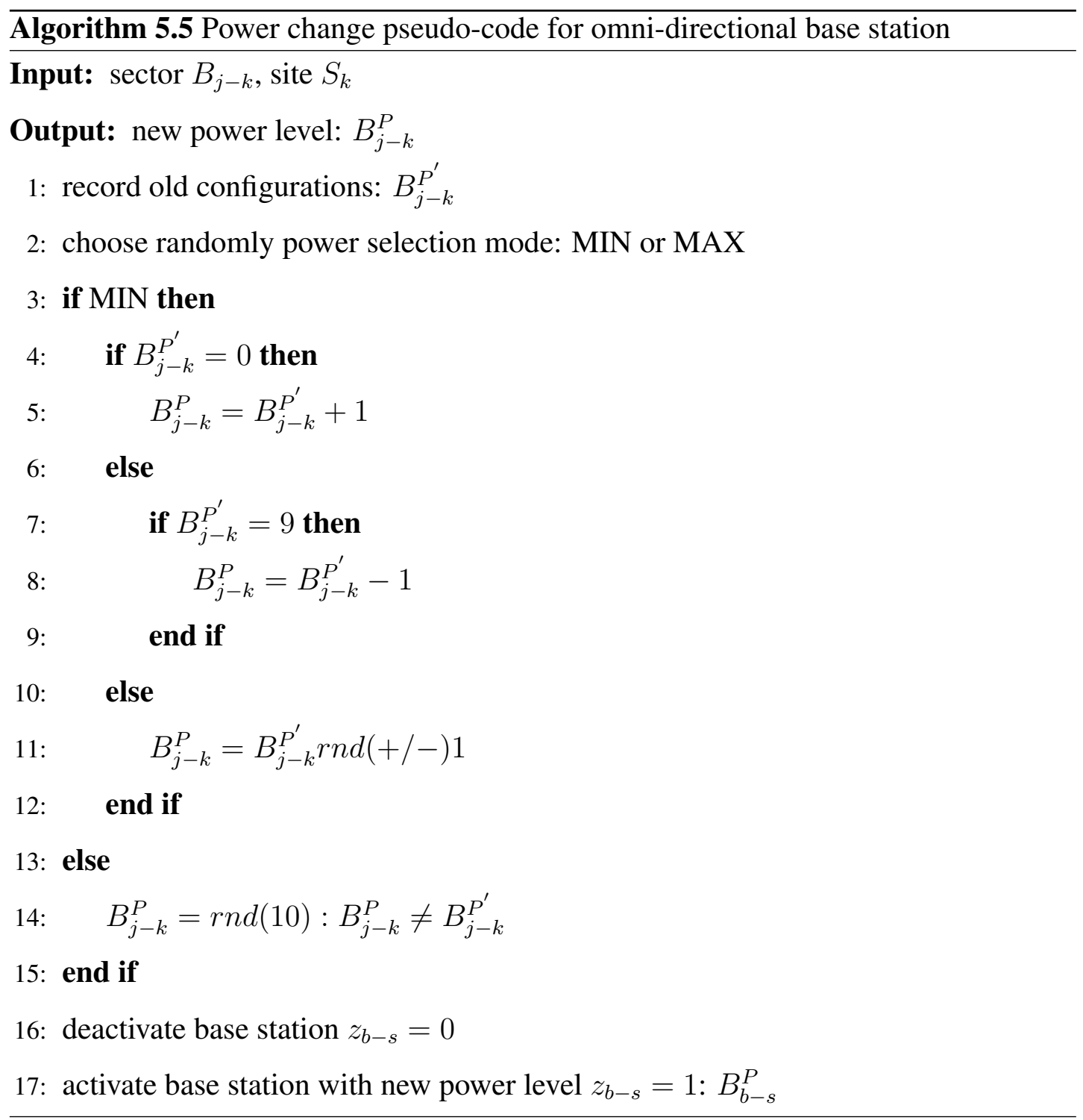

show the original pattern, figures 5.9 and 5.10 present the pattern of omni-directional antenna tilted by 14 degrees.

V. Move BS - this procedure is very useful in terms of exploring the search space, since the use of such modification can make considerable alteration to the current solution. This modifier significantly improves and helps the algorithm to avoid getting stuck in local optima. The procedure is realised by maintaining the lists of inactive neighbours for all active sites arranged in descending order of distance from the selected site. Once the site is selected for movement the list of neighbours is investigated. If there 


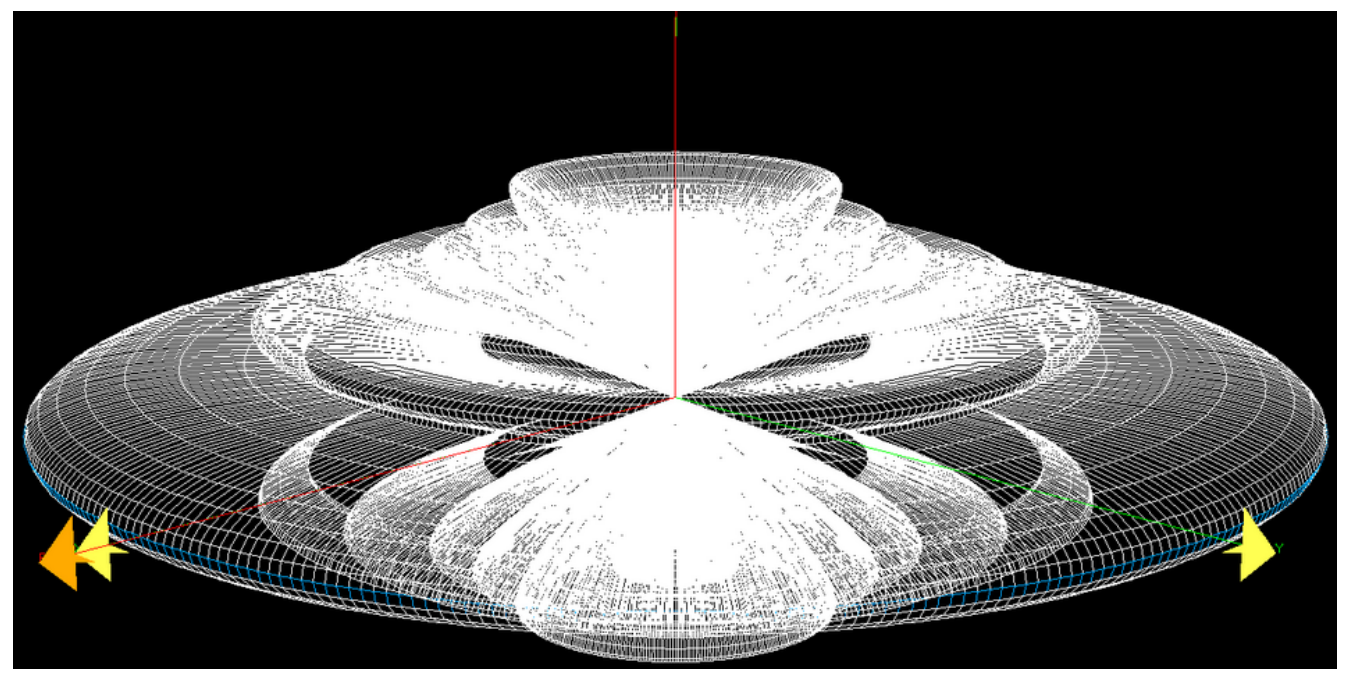

Figure 5.7: Original omni-directional pattern - angled view

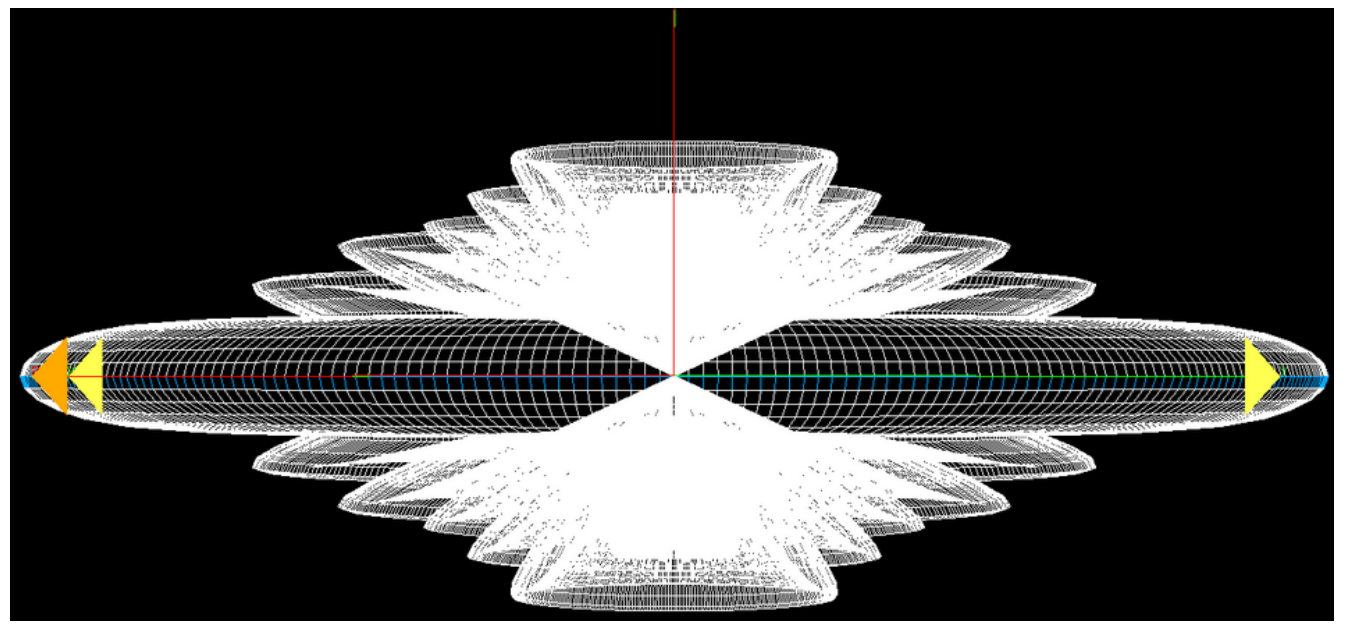

Figure 5.8: Original omni-directional pattern - side view

are no neighbours available, which means that all the sites are active then the base station at the selected site is transformed by changing the power and the tilt to random values. The list of considered neighbours does not exceed 6 site locations. The new site is chosen randomly. The limit of six neighbours is dictated by the need to explore the closest neighbourhood rather than moving the base station from one corner of the network to opposite one. After the site is chosen the previous site is deactivated and the new site is deployed with default configurations. 


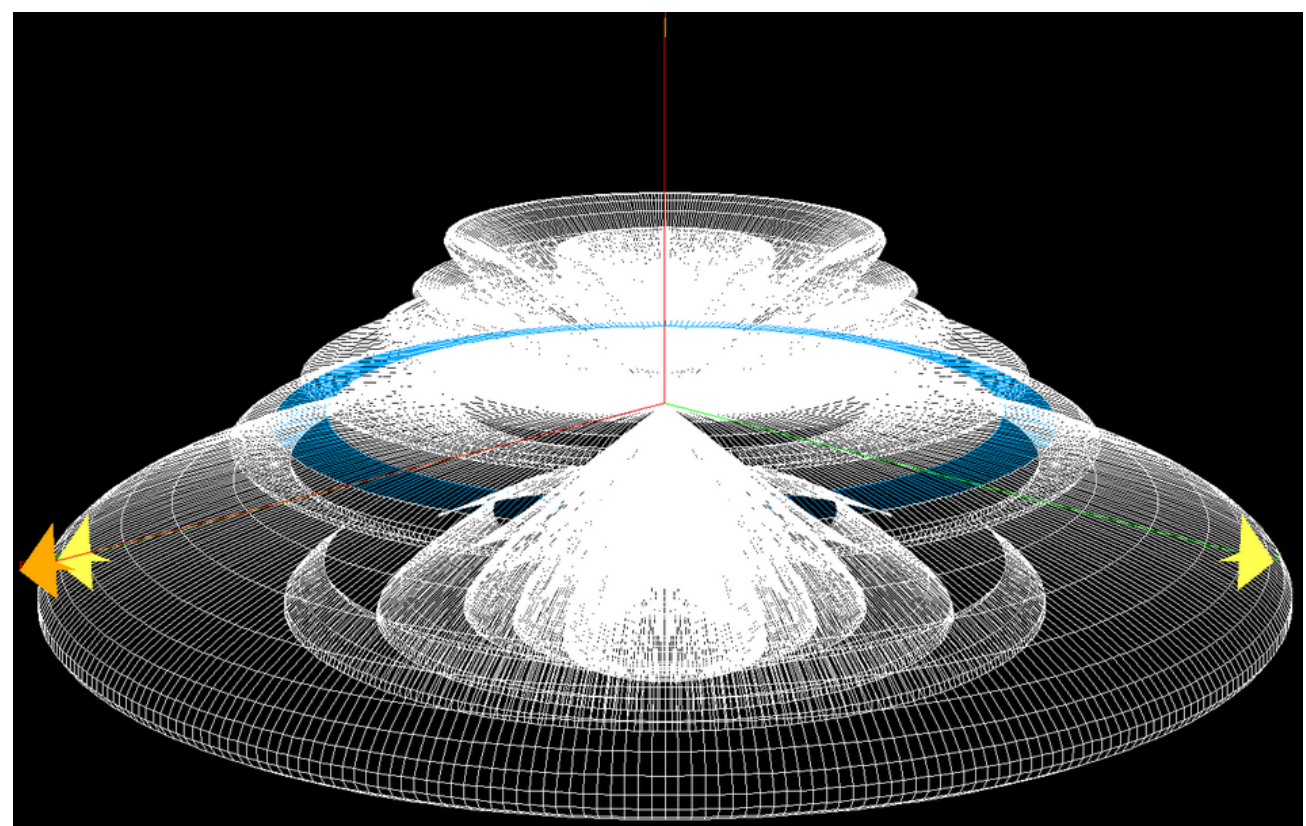

Figure 5.9: Tilted omni-directional pattern (14 degrees) - angled view

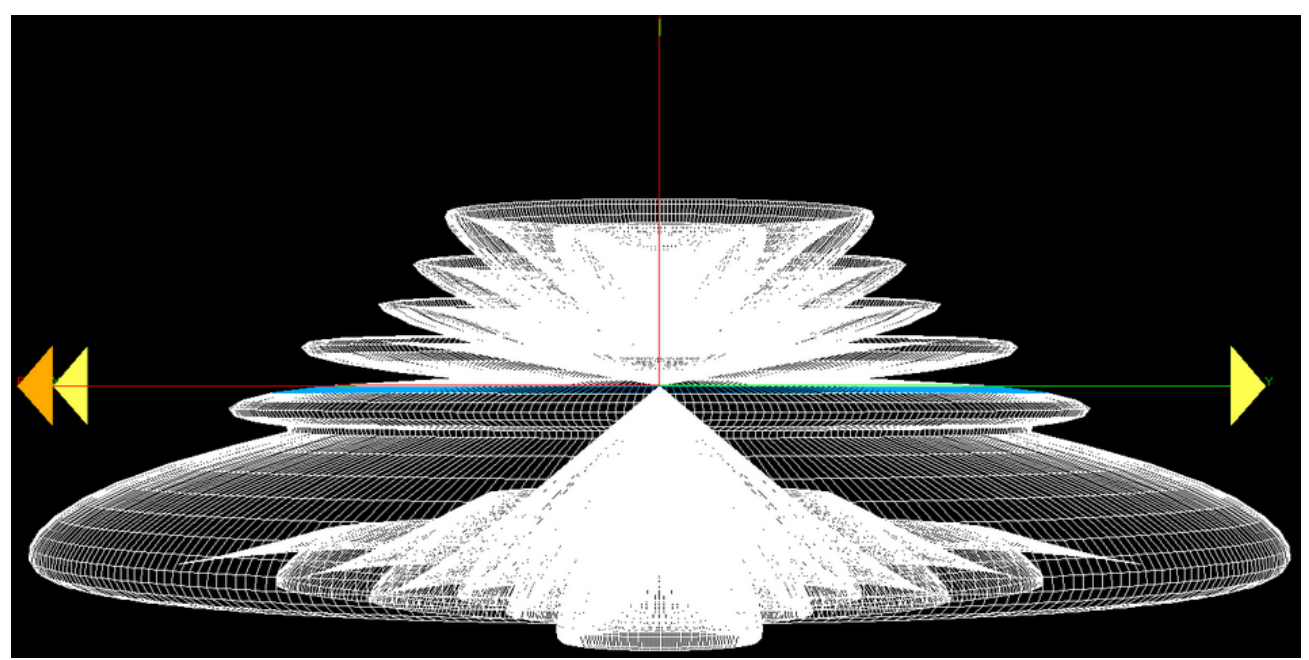

Figure 5.10: Tilted omni-directional pattern (14 degrees) - side view

VI. Change capacity - the capacity amendment for an omni-directional base station emulates the activation or deactivation of an additional sector with a directional pattern. Therefore, an increase in capacity is reflected by the increase of available slots for user assignment and an increase in the base station cost. Hence, the aim of this modifier is to find an optimal balance between the gain in ability to serve more users (and therefore 


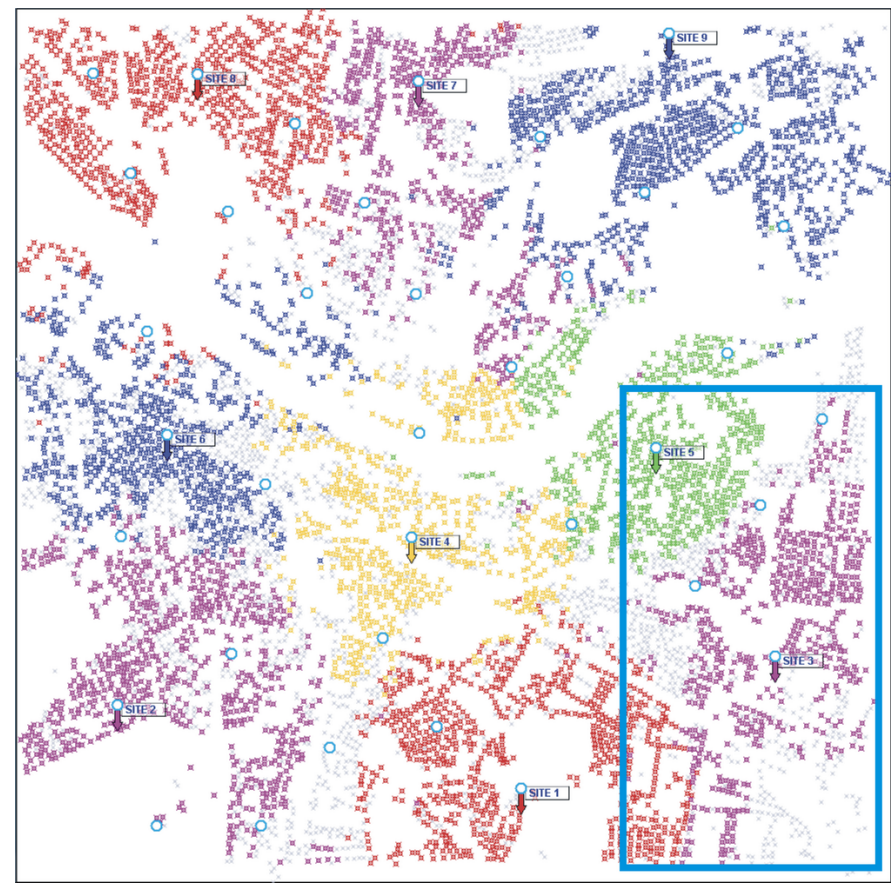

Figure 5.11: Base station '3' with capacity of 6 sectors

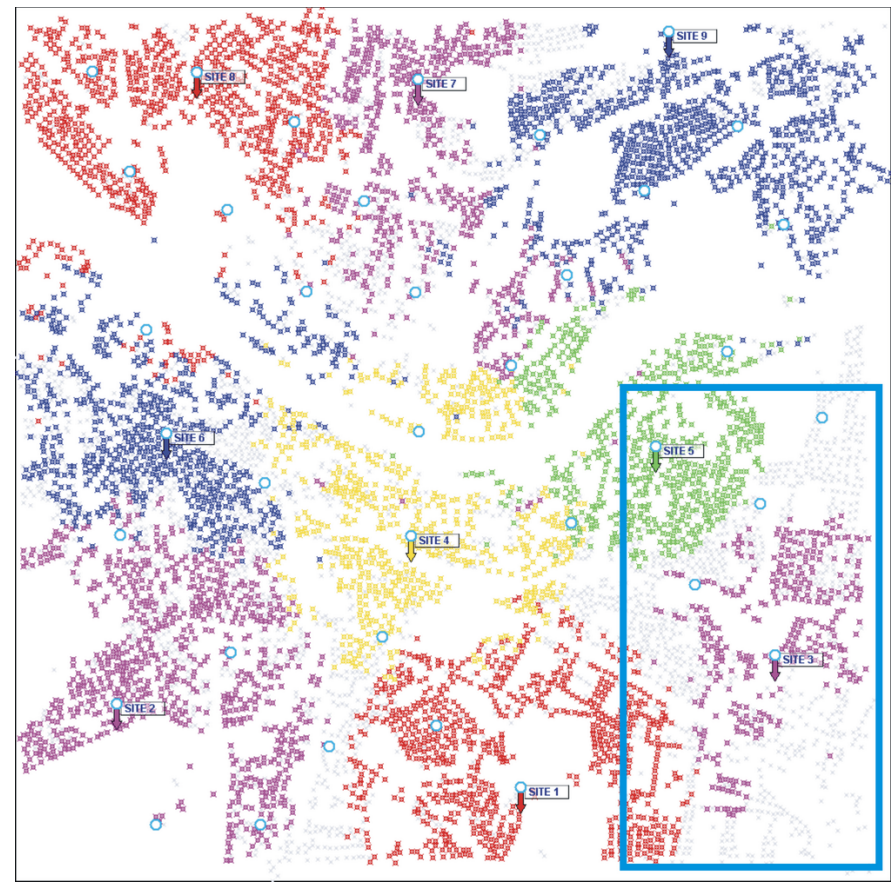

Figure 5.12: Base station ' 3 ' with capacity of 2 sectors 


\begin{tabular}{|c|c|c|c|c|}
\hline Sites & Site ID & Capacity & Serviced users before & Serviced users after \\
\hline 1 & 2 & 6 & 754 & 771 \\
2 & 5 & 6 & 713 & 757 \\
$\mathbf{3}$ & $\mathbf{6}$ & $\mathbf{6}$ & $\mathbf{6 3 8}$ & $\mathbf{3 2 8}$ \\
4 & 10 & 6 & 626 & 655 \\
5 & 15 & 6 & 622 & 637 \\
6 & 16 & 6 & 687 & 693 \\
7 & 33 & 6 & 505 & 524 \\
8 & 34 & 6 & 826 & 834 \\
9 & 36 & 6 & 848 & 844 \\
\hline
\end{tabular}

Table 5.4: Variation in the number of serviced users before and after changing the capacity of BS from 6 to 2 sectors in Stage 1.

generate more revenue), and on the other hand the increase of expenditure to realise the additional capacity. The modification of capacity is performed in the same way as 'change power' with the difference in limits - minimum capacity of 2 sectors and maximum of 6 . The effect of changing the capacity of a base station can be seen in figures 5.11 and 5.12 , and Table 5.4 .

The comparison of move selection strategies, dynamic and fixed (when the probabilities of selecting moves are equal) is presented in Figure 5.13. The graph represents the normalized values of the acceptance ratio of moves. The 'change capacity' modifier value in the dynamic strategy is the most successful performer. It can be seen that in both selection strategies the relative ratio between the moves is approximately the same, with 'deactivate site' having the lowest acceptance ratio and 'change capacity' the highest. However, the dynamic mechanism doubles (or more for some moves) the performance ratio, or in other words with the dynamic evaluation of each moves performance it sets probabilities for selecting the modifier in a way that it gets accepted more frequently than in a fixed mode. 


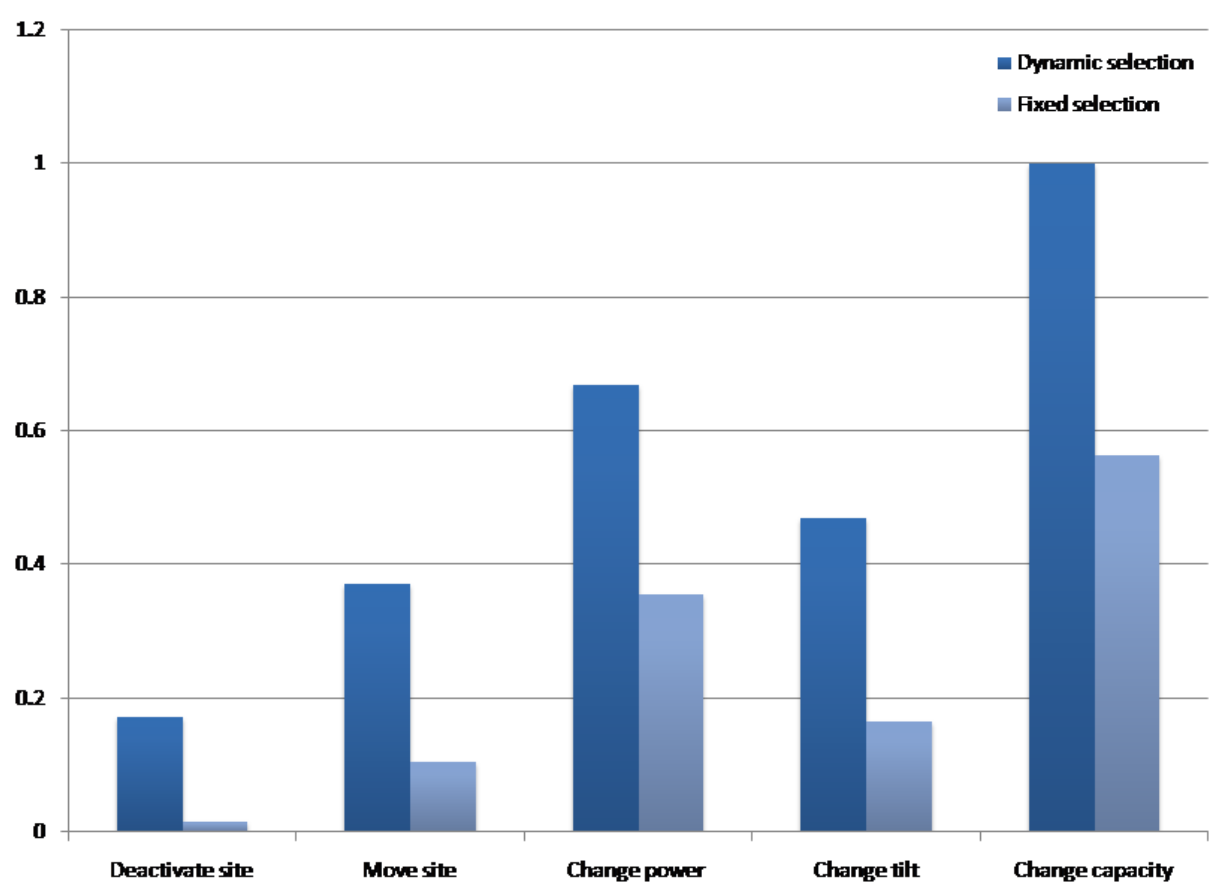

Figure 5.13: Normalized acceptance ratios of moves for dynamic and fixed strategies.

\subsection{Stage objectives and cost function weights}

Generally, the initial solution or starting point in the optimization process plays an important role. The better the starting solution the smoother and quicker the proceeding process can reach the global optima. In our case the result of Stage 1 provides the starting point for the following stages of sectorizing and re-configuring the base stations. Therefore, it can be said that it is not only the starting point but it also generates direction for the stages ahead. Hence, stage 1 must target the most crucial objectives which define the chosen quality of solution for the service provider. The objectives considered for this stage and their weights in the cost function calculation are:

$\begin{array}{ll}\text { coverage maximization } & 0.1 \\ \text { service maximization } & 0.6 \\ \text { traffic unit cost minimization } & 0.3\end{array}$ 
The main emphasis is on finding solutions with maximum service level and low traffic unit cost which in a sense represent the generated profit of the network. The coverage is not weighed at a greater level as maximization of the service guaranties high level of coverage. The weights have been chosen through a series of experimental runs.

\subsection{Output of Stage 1}

Having addressed all the issues related to Stage 1, defined all the moves and objectives we can finally present the output of the stage for subsequent use by stage 2 :

1. A set of optimal site locations with activated base stations to satisfy all the objectives imposed at the stage.

2. A set of users in service for each activated base station.

3. A number of sectors required for each base station to provide service for the given areas.

Figure 5.14 and Table 5.5 provide a sample of the resulting output solution of Stage 1. Table 5.6 provides the Key Performance Indicators (KPI) for the same network instance.

The framework of Stage 1 in some respect provides a network dimensioning tool for WiMAX networks. Network dimensioning techniques are generally used to provide a quick estimate of the network's capabilities by making the necessary estimations and assumptions, and more importantly without going into a full optimization process. There is a number of published dimensioning methodologies for WCDMA technology, one of which provides a good analysis [115] for existing techniques. In [115] the author lists several approaches such as coverage dimensioning and capacity dimensioning, which when combined must provide an estimate of the number of sites for selection 


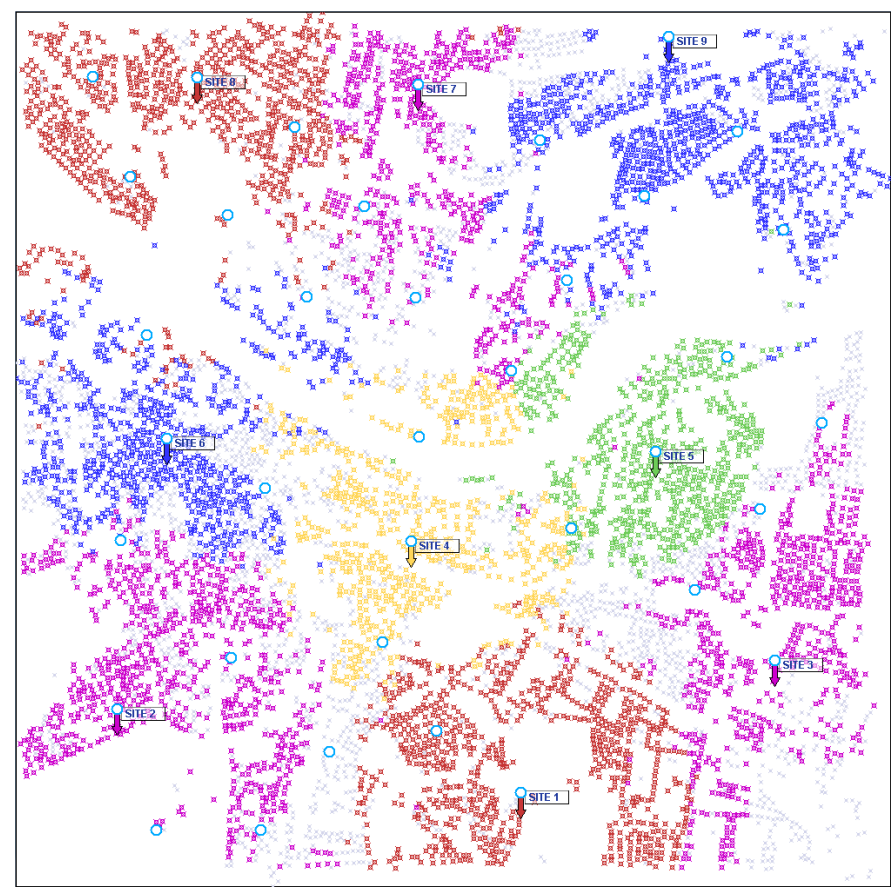

Figure 5.14: Stage 1 network output display

\begin{tabular}{|c|c|c|c|}
\hline Sites & Site ID & Capacity & Serviced users \\
\hline 1 & 2 & 6 & 754 \\
2 & 5 & 6 & 713 \\
3 & 6 & 6 & 638 \\
4 & 10 & 6 & 626 \\
5 & 15 & 6 & 622 \\
6 & 16 & 6 & 687 \\
7 & 33 & 6 & 505 \\
8 & 34 & 6 & 826 \\
9 & 36 & 6 & 848 \\
\hline
\end{tabular}

Table 5.5: Network results breakdown after Stage 1

and their initial configurations, in order to provide a satisfactory service and to meet required capacity specifications. 


\begin{tabular}{|l|l|}
\hline Key performance indicator & Values \\
\hline Coverage level $(\%)$ & 98.28 \\
Service level $(\%)$ & 72.46 \\
Network cost $(€)$ & 432000 \\
Profit $(€)$ & 187200 \\
Traffic unit cost (€/Kbps) & 0.347 \\
Spectral efficiency (bps/Hz) & 1.15 \\
Capacity $(\mathrm{Mbps})$ & 1243 \\
\hline
\end{tabular}

Table 5.6: Key performance indicators of Stage 1

The contribution of our approach is that Stage 1 not only identifies the required number of sites and their possible configurations, but more importantly the locations of these sites. Our model takes into account the full complexity of the network including traffic distribution, interference modelling and financial components of the network. The results produced can be used for initial budgetary estimation purposes for the network provider or as the starting cell plan for the subsequent automated cell planning process. 


\section{Chapter 6}

\section{Optimization - Stage 2 \& Stage 3}

\subsection{Stage 2. Sectorization}

Having identified the site locations and the provisional sets of users to be serviced by the base stations at these sites, Stage 2 of the optimization framework takes control of the preliminary sectorization of the omni-directional antennas with the number of sectors defined in Stage 1, and configuration of each sector for coverage and service. The main goal of Stage 2 is to quickly find the required settings for the base stations in order to get results close enough to the ones obtained from Stage 1. In comparison to Stage 1 where a site can be selected for modification more than once over the entire optimization process, in Stage 2 all sites are sectorized and undergo a series of modifications only once. The order in which sites are sectorized is from the highest traffic load to the lowest.

The overall procedure of Stage 2 is depicted in Figure 6.1 and these steps can be defined as follows:

1. sort sites in descending order of traffic load - the first site from the list is sectorized, then algorithm progresses through the rest of the list;

2. from each site obtain the list of serviced users and capacity in terms of the number of sectors - these characteristics define the cell size and shape which must be serviced by the sectors; 
3. deactivate/remove all sites from the network - the sectorization process starts on a clean network instance, with sectorized sites joining during the optimization process;

4. identify the traffic centre of mass for each cell - this defines the centre of a cell in terms of traffic;

5. sectorize each cell with calculated initial azimuths - the sites are not sectorized with uniform separation between them but taking into account the traffic mass centre;

6. perform a range of modifications - each sectorized site undergoes a series of randomly selected modifications to improve the imposed objectives.

The critical point of this stage is to obtain good results in a short amount of time, hence the strategy of targeting the specified goals in the most effective way. All the operations are described in full in the following sections.

\subsubsection{Optimization algorithm}

The fact that results obtained from Stage 2 do not require optimality as it is an intermediate stage where the base stations are sectorized and approximately configured in a 'cell by cell' sequence. A solution is desired to be derived in a short amount of time, hence the choice of Hill Climbing ( $\mathrm{HC})$. The pseudo-code for $\mathrm{HC}$ is provided in Algorithm 6.1.

In contrast with SA, hill-climbing always attempts to make changes that improve the current state. In other words, hill-climbing can only advance if there is a lower point (minimization problem) in the adjacent landscape. Therefore, the main problem that hill-climbing can encounter is that of local minima. This occurs when the algorithm 


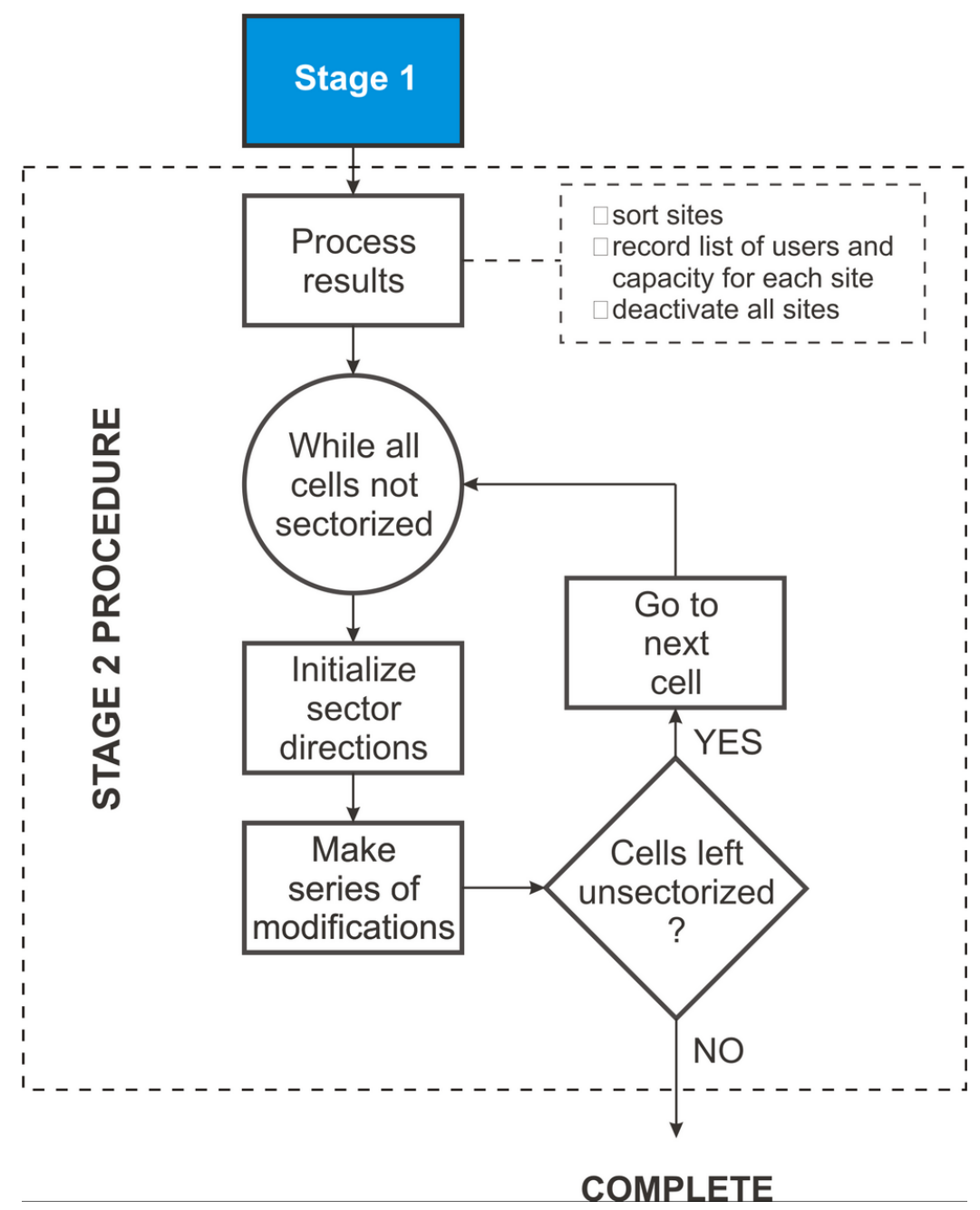

Figure 6.1: Stage 2 procedure

stops making progress towards an optimal solution; mainly due to the lack of immediate improvement in adjacent states. However, as it has been mentioned before the issue of HC getting stuck in local minima is not a concern as:

- the main goal of this stage is to get a good enough solution as quickly as possible,

- the local minima each solution converges to with hill-climbing is only local to a cell instance which is different to a network local minima.

With each omni cell being sectorized, the output it provides becomes an input for the next cell in the list. The stopping criteria for the algorithm is chosen to be a number of 


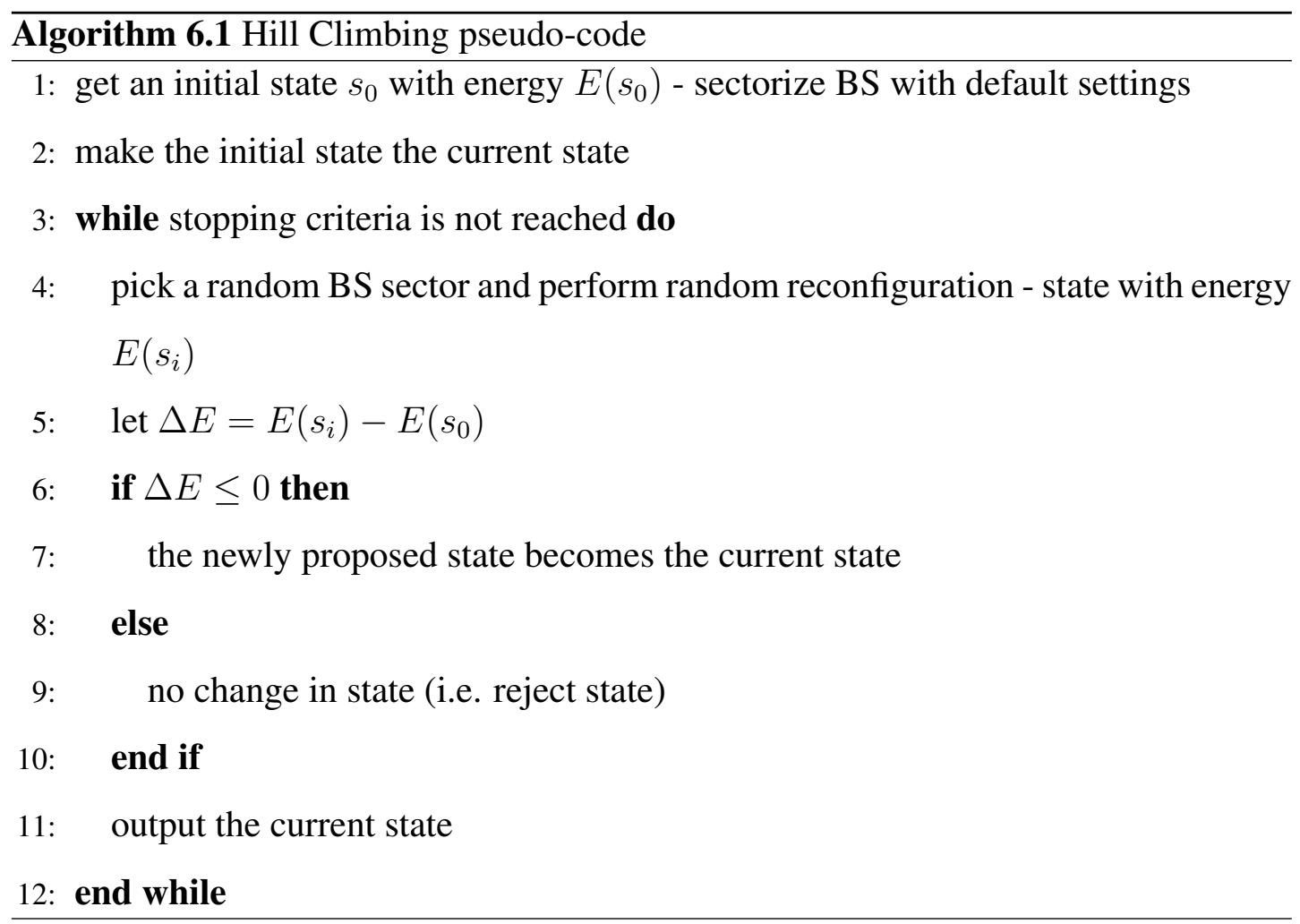

states without improvement (set to 700 steps).

\subsubsection{Initial sectorization of cells}

As it can be seen from Figure 5.14 the shapes of the cells constitute the irregular forms in comparison to the ideal representation of a cell with a hexagonal shape. This can be explained by the range of affecting factors such as varying propagation losses between sites and users, non-uniform distribution of users throughout the network space, interference between the cells etc. Having irregular shaped cells, the sectors must be positioned in such a way so that the users within a cell receive the maximum signal quality from their servers and the sectors achieve the most efficient traffic load. This cannot be achieved by separation of sector antennas with equal angles in the horizontal plane, e.g. six sectors with 60 degrees separation. In this case in order to identify the correct targeting directions for each sector the algorithm will need to sample variations of azimuths for antennas which will consume a considerable amount of time. Instead, 
the distribution of users can be thoroughly analysed to produce the required positioning of antennas.

First, the algorithm evaluates the traffic demand distribution within the given region relative to the site position. This is done in order to calculate a 'centre of mass', also known in physics as a 'centre of gravity', which can be defined as:

Definition 6.1.1. The centre of mass of a system of particles, $\boldsymbol{R}$, of total mass $M$ is defined as the average of their positions, $R_{i}^{x, y}$, weighted by their masses, traffic demand in our case, $R_{i}^{\tau}$ :

$$
\mathbf{R}_{\mathbf{s}}=\frac{1}{M} \sum R_{i}^{x, y} \times R_{i}^{\tau}
$$

This calculation will produce a mass centre point which will give an initial direction for the sectors. However, the next step is to identify how to distribute the sectors, which greatly depends on the distance between the mass centre and the site's geographical position, as well the amount of sectors to be deployed.

The initial sectorization of an omni-directional cell, Figure 6.2, is performed using the following stages:

1. Calculate the centre of mass for the distribution of traffic among the cell, (Figure 6.3);

2. Calculate the distance, $d_{\max }$, to the furthest serviced user from the site location in the direction of mass centre;

3. Calculate the angle, $\alpha_{y}$, between the site position and the centre of traffic mass which forms a vertical axis of the sectorized cell;

4. Identify the perpendicular line to the vertical axis, $\alpha_{x}$;

5. Partition users on two zones - below the horizontal axis of mass centre $\alpha_{x}$ (red zone 1) and above (blue zone 2), Figure 6.4; 


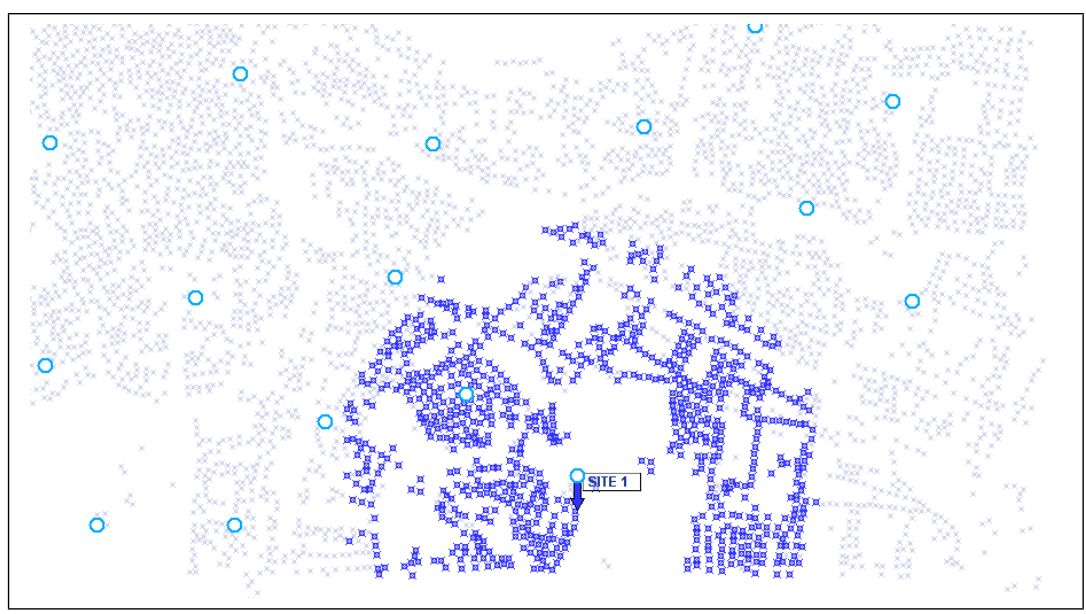

Figure 6.2: Omni cell structure before sectorization

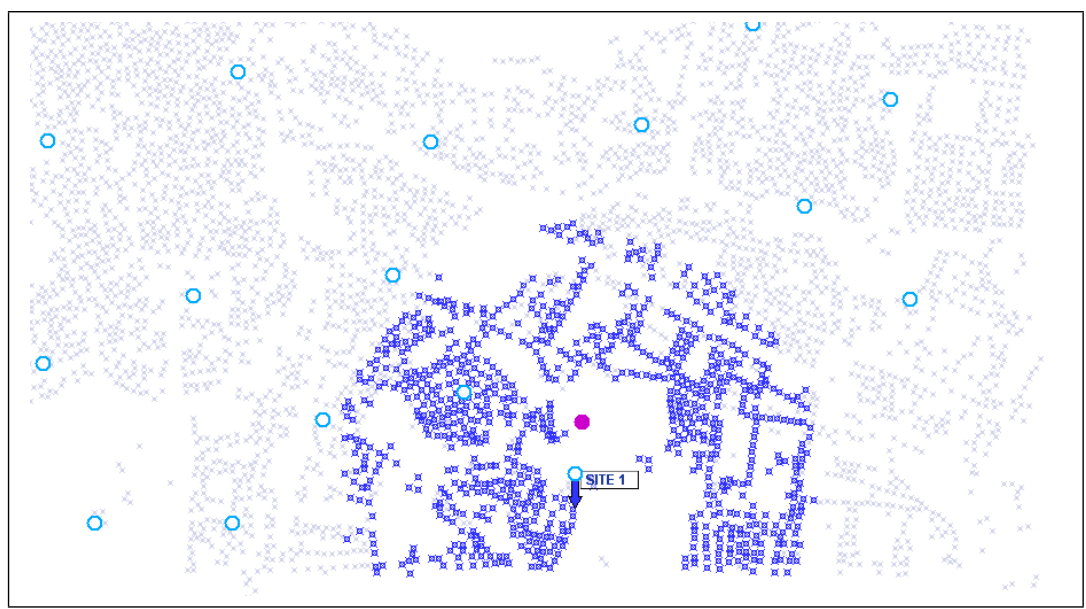

Figure 6.3: Identifying the centre of mass for the omni cell

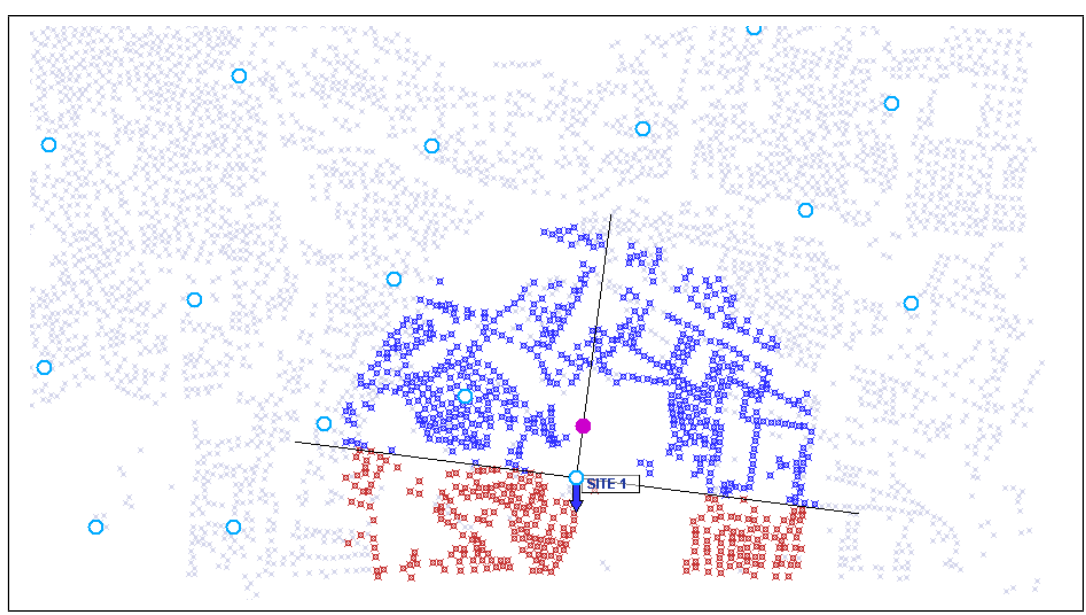

Figure 6.4: Partitioning the omni cell on two zones 
6. Determine the amount of sectors required to serve each zone by the ratio of zone users to the total number of users:

$$
\begin{gathered}
\operatorname{sectors}\left(\text { zone }_{1}\right)=\operatorname{round}\left(n_{\text {users }}^{\text {zone }} / n_{\text {users }}^{\text {total }}\right) \times B^{\text {cap }} \\
\operatorname{sectors}\left(\text { zone }_{2}\right)=B^{\text {cap }}-\operatorname{sectors}\left(\text { zone }_{1}\right)
\end{gathered}
$$

7. The further the centre of mass is from the site location the less users are positioned in zone 1, and therefore the less sectors the base station requires to serve the users within the zone. For this stage of optimization in order to target antenna patterns most efficiently the azimuth range each antenna can explore is limited and we introduce the following notations: zone range - which defines the angle sector in the horizontal plane available for sectors of the zone to operate on; antenna base angle - which defines the central (and initial) azimuth position for each antenna; sector diapason - the azimuth diapason in which each sector can operate around the base angle. These notations can be found on Figure 6.5 where $\mathbf{A}$ is a zone range, $\mathbf{B}$ is a sector diapason (60 degrees in this example) defining a range of azimuth values for the antenna direction, and base angle $\mathbf{C}$ set to 270 degrees:

$$
\begin{gathered}
\mathbf{A}_{\mathbf{1}}=\left\{\begin{array}{l}
180 \text { degrees }- \text { if more than } 1 \text { sector in zone } 1 \\
120 \text { degrees }- \text { otherwise }
\end{array}\right. \\
\mathbf{B}_{\mathbf{i}}=\frac{\mathbf{A}_{\mathbf{1}}}{\text { sectors }(\text { zone } 1)} \\
\mathbf{C}_{\mathbf{i}}=\left(180-\alpha_{y}\right)-\frac{\mathbf{A}_{\mathbf{1}}}{2}+\mathbf{B}_{\mathbf{i}} \times\left(\frac{1}{2}+i\right)
\end{gathered}
$$

All these settings are set for each sector, $i: 0-2$, in order to reduce the amount of decision variables for the algorithm to choose from. Zone 1 is initialized first. If the number of sectors needed is more than 1 then the whole 180 degrees zone range is used for sectors to work on, otherwise only 120 degrees is allocated. 


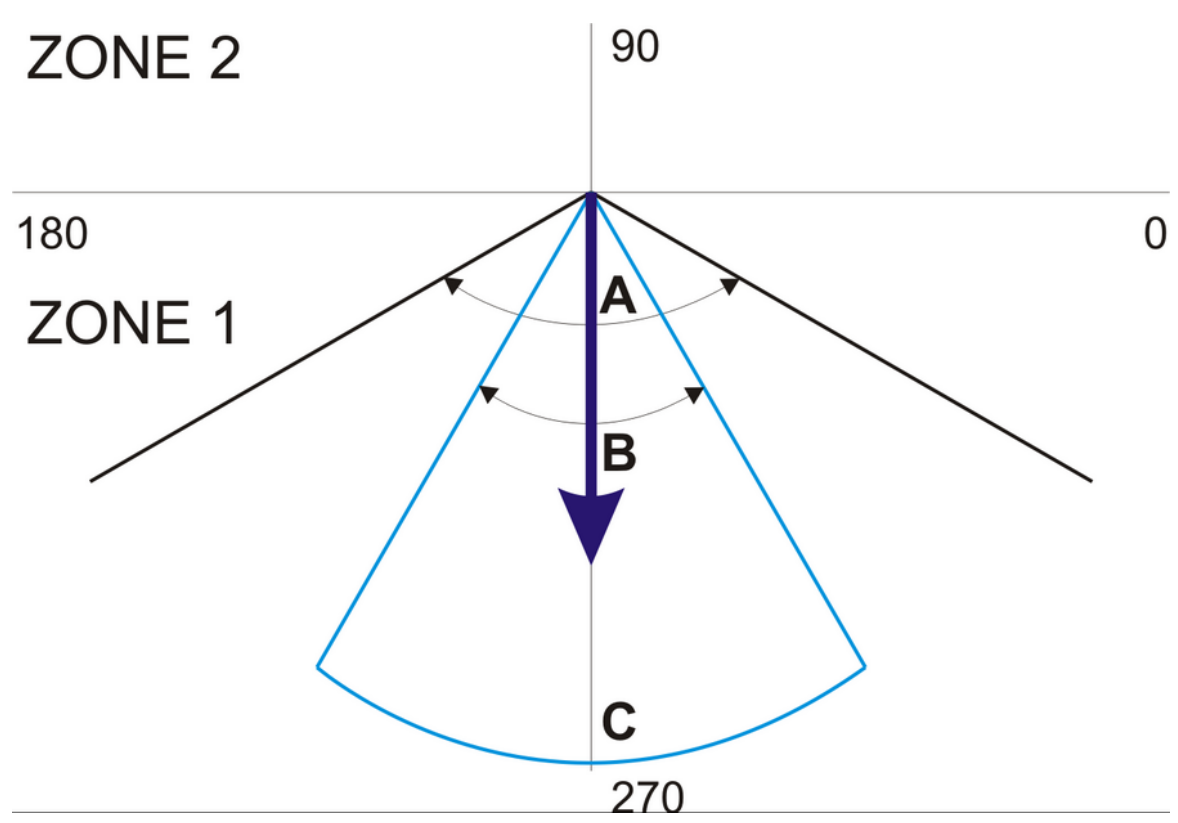

Figure 6.5: Azimuth limitations of zone 1 in sectorization process

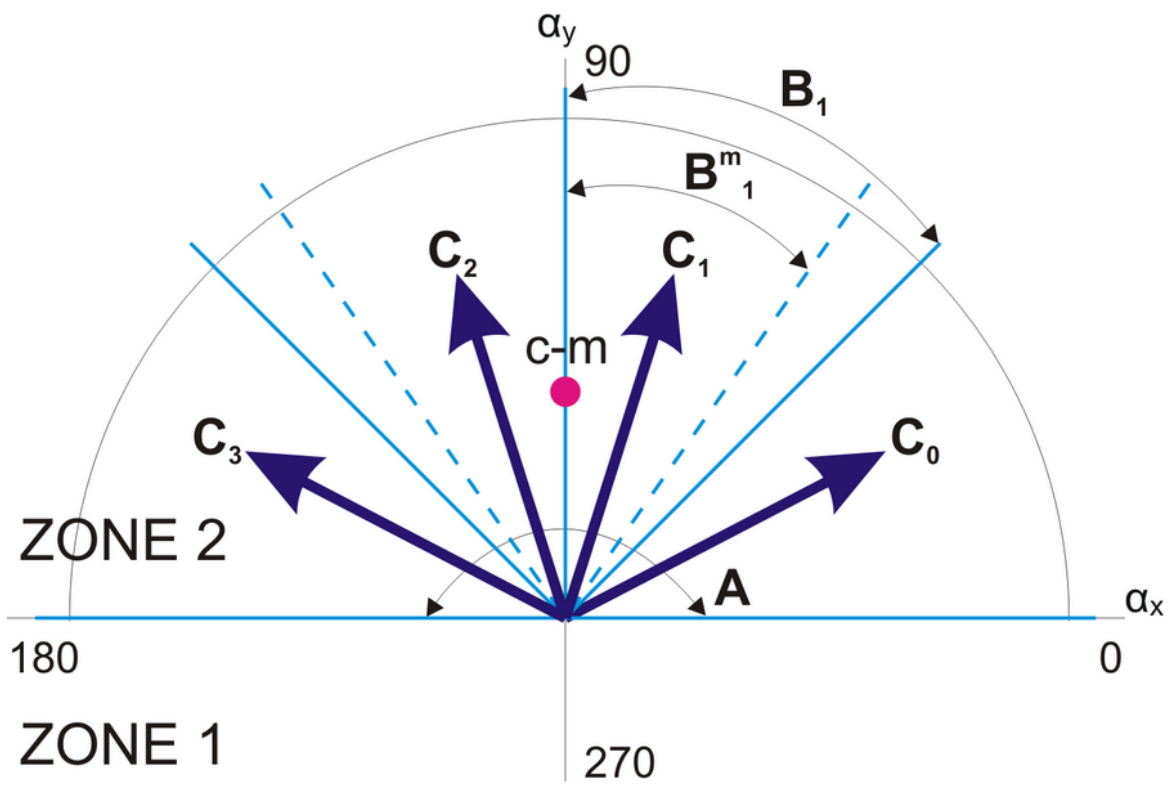

Figure 6.6: Azimuth limitations of zone 2 in sectorization process

8. Zone 2 partitioning is the final stage of sector initialization. Unlike in zone 1 the zone range of zone 2 is always greater or equal to 180 degrees as it always incorporates the majority of users within the entire cell: 


$$
\mathbf{A}_{\mathbf{2}}=360-\mathbf{A}_{\mathbf{1}}
$$

However the procedure on sectorizing zone 2 differs depending on the number of sectors allocated to it, in particular whether an even or odd number. First let's describe the even case, Figure 6.6. The zone range is partitioned into sectors with equal sector diapasons:

$$
\mathbf{B}_{\mathbf{i}}=\frac{\mathbf{A}_{\mathbf{2}}}{\operatorname{sectors}\left(\text { zone }_{2}\right)}
$$

As the distance to the centre of mass represents the shape of the cell, i.e. how the cell is stretched in this direction (the greater the distance the more parabolic shape it takes), hence the more users are positioned in this direction. Therefore the partitioning of sectors must reflect this factor:

$$
\begin{gathered}
k_{\text {mass }}=\frac{d_{\text {mass }}}{d_{\text {max }}} \\
\mathbf{B}_{(\mathbf{1 , 2})}^{\mathbf{m}}=\mathbf{B}_{(\mathbf{1}, \mathbf{2})} \times\left(1-k_{\text {mass }}\right) \\
\mathbf{B}_{(\mathbf{0}, \mathbf{3})}^{\mathbf{m}}=\mathbf{B}_{(\mathbf{0 , 3})} \times\left(1+k_{\text {mass }}\right)
\end{gathered}
$$

Expressions 6.9 and 6.10 modify the sector diapasons for sectors 1 and 2 by shrinking them by a factor of $1-k_{\text {mass }}$ and as the main mass of users is concentrated in the direction of the centre of mass, sectors 0 and 3 are expanded by the factor $1+k_{\text {mass }}$ respectively. It is clear that the further the centre of mass the greater difference between the two, and vice versa.

The base angles for the sectors in zone 2 are also modified according to the factor of the distance to the mass centre: 


$$
\begin{gathered}
\mathbf{C}_{(\mathbf{1}, \mathbf{2})}=\alpha_{y}+/-\frac{\mathbf{B}_{(\mathbf{1}, \mathbf{2})}^{\mathrm{m}}}{2} \\
\mathbf{C}_{(\mathbf{0}, \mathbf{3})}=\alpha_{y}+/-\frac{\mathbf{B}_{(\mathbf{0}, \mathbf{3})}^{\mathrm{m}}+2 \times \mathbf{B}_{(\mathbf{1}, \mathbf{2})}^{\mathrm{m}}}{2}
\end{gathered}
$$

The $+/-$ sign in expressions 6.11 and 6.12 is given to reflect the position of antenna in relation to the vertical axis $\alpha_{y}$, - if on the right side, and + for the opposite side.

The process for sectorizing a zone with an odd amount of sectors is very similar to the one described, the only difference being that the central sector is positioned first on the line of vertical axis and then the parameters sector diapason and base angle are calculated for the rest of the sectors.

An example of the initial sectorization can be seen in Figure 6.7. With the majority of users positioned in zone 2 the algorithm allocated only 2 sectors to zone 1 which can be seen is more than enough to service the users. Zone 2 is more populated and stretched, which reflects in sectors marked with green and red colours being more closely directed to each other, and sectors marked with blue and black colours having higher sector diapasons.

The same site after a series of modifications can be seen on Figure 6.8. The origin of the axis starts from the left top corner, with the 'y' axis directed to the south (bottom left corner) and the ' $x$ ' axis to the east (top right corner). Therefore, the ' 0 ' degrees azimuth is in the direction from the cell centre to the south, '90' degrees azimuth to the east etc. Initially all the sectors are activated with default configurations:

- slot power level - $17 \mathrm{dBm}$

- antenna type - 60 degrees pattern

- tilt - 10 degrees 


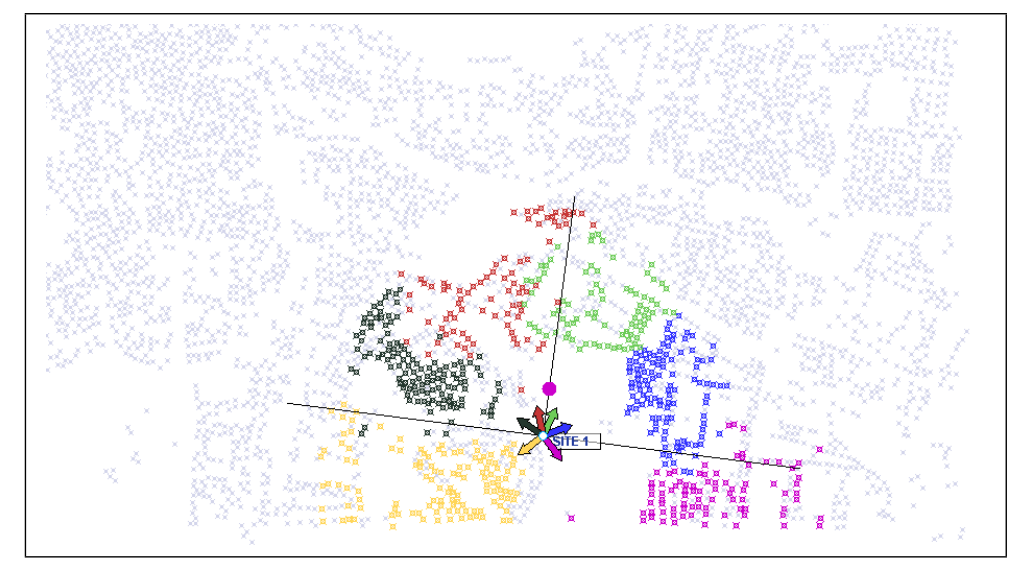

Figure 6.7: Initial sectorization of omni base station with default configurations

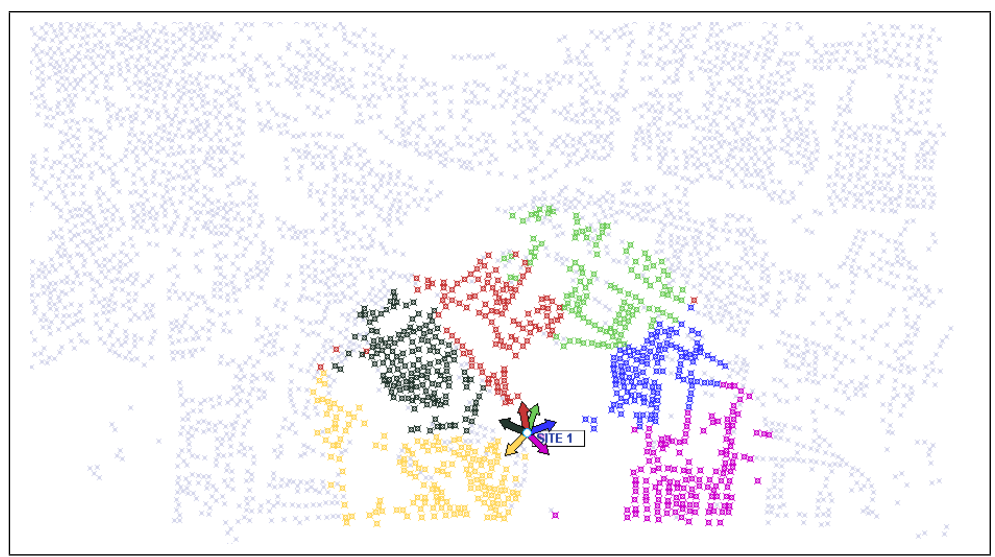

Figure 6.8: The resulting sectorized cell after a series of modifications

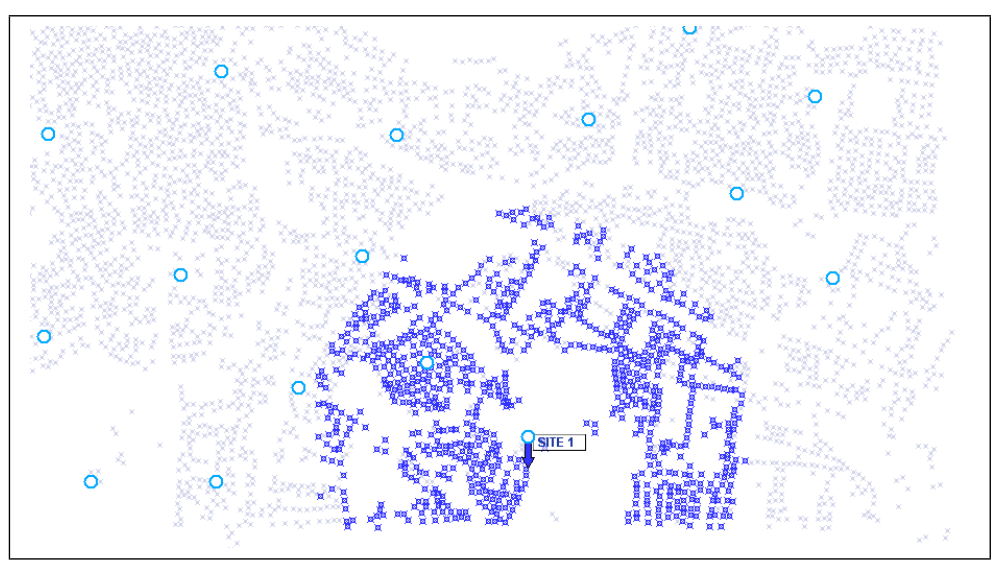

Figure 6.9: Comparison of omni cell structure to sectorized solution 
However, after the moves are applied (described in the following section) the resemblance of the overall cell structure to the one provided from the Stage 1, Figure 6.9, is significant. This also can be seen from the performance indicators provided in Table 6.1.

\begin{tabular}{|l|c|}
\hline KPI & Values \\
\hline Served users Stage 1 & 861 \\
Served users Stage 2 & 811 \\
Stage 2 assignment ratio & 0.91 \\
\hline
\end{tabular}

Table 6.1: Performance comparison of Stage 1 and Stage 2 for a cell

The assignment ratio is a parameter which reflects the ratio of users (from the initial list provided by Stage 1) serviced in Stage 2 to the total amount of users from this list. Which means that only 27 users out of 811 are not from the initial list.

Table 6.2 shows the changes in sector parameters after the algorithm performed the re-configurations (presented in Figure 6.8) using 515 iterations. In the table I indicates the sector parameters at the starting configuration using the default values, an $\mathbf{O}$ is for the optimized settings on completion of Stage 2.

\begin{tabular}{|c|c|c|c|c|c|c|c|c|}
\hline \multirow{2}{*}{ Sectors } & \multicolumn{2}{|c|}{ Power $(\mathbf{d B m})$} & \multicolumn{2}{c|}{ Azimuth } & \multicolumn{2}{|c|}{ Tilt } & \multicolumn{2}{c|}{ Type } \\
\cline { 2 - 9 } & I & O & I & O & I & O & I & O \\
\hline 1 & 17 & 27 & 308 & 316 & 10 & 12 & 60 & 60 \\
\hline 2 & 17 & 27 & 38 & 45 & 10 & 11 & 60 & 60 \\
\hline 3 & 17 & 17 & 110 & 110 & 10 & 10 & 60 & 30 \\
\hline 4 & 17 & 17 & 155 & 161 & 10 & 7 & 60 & 120 \\
\hline 5 & 17 & 19 & 191 & 190 & 10 & 12 & 60 & 30 \\
\hline 6 & 17 & 19 & 236 & 246 & 10 & 11 & 60 & 30 \\
\hline
\end{tabular}

Table 6.2: Re-configuration of sectors in Stage 2 for one cell

From the table it can be seen that the relative azimuth separation between the sectors has been retained with only minor corrections. This shows that the algorithm makes 
less computational effort in finding the optimal azimuth directions for antennas which indicates the importance of the initial sectorization phase.

\subsubsection{Operational moves}

In comparison to Stage 1 where the next modification in the algorithm process is applied to a randomly selected site according to the move selection strategy, in Stage 2 the algorithm optimizes each site with a random selection of sectors instead. Therefore, at each iteration of the algorithm a random re-configuration modification is employed to a randomly selected sector. These moves are listed as follows:

1. Change power - this move is performed in a similar way as in Stage 1 with a variation in the way the power level can be changed. The pseudo-code is presented in Algorithm 5.5 .

2. Change azimuth - is a modifier with the highest number of choices available compared to other moves. Hence, the limitations in azimuth exploration imposed in Section 6.1.2 are put in place in order for sectors to most effectively target the traffic demand of users distributed within a cell. After the initial sectorization each sector maintains two parameters throughout the entire Stage 2 process - base angle and sector diapason, which define the central azimuth position of the antenna and the azimuth diapason of the antenna respectively. Both of these parameters essentially provide the minimum and maximum azimuth directions for the algorithm to sample during the optimization operation. For example, if as a result of initial sectorization the sector has been set with 210 degrees base angle and 60 degrees sector diapason, it translates into a minimum azimuth of 180 and maximum of 240 degrees. The algorithm then randomly selects the new azimuth within the two limits. Therefore, the approach of initial sectorization is aimed at reducing the range of azimuth directions for the areas of high user concentration in order to reduce the effort for the algorithm to identify other sector configurations for effective service of these areas. 
3. Change tilt - in comparison to Stage 1 where the tilt modification of an omnidirectional antenna is artificial, the same modification in Stage 2 is with real directional antenna patterns which are designed to be tilted, mechanically or electronically. The amendment of tilt angle results in the change of antenna vertical losses in calculation of downlink signal strength described in Section 3.3. This modifier is implemented in the same way as the 'Power change' move but with 15 degrees of variation for tilt.

4. Change antenna type - the choice of antenna patterns used in the thesis has been limited to those with $30,60,90$ and 120 degrees radiation patterns. When this move is selected for modification of a sector the antenna pattern is chosen randomly from the given list of available types.

\subsubsection{Base station PermBase allocation procedure}

PermBase is an integer used in the permutation formulas in Section 3.5 .3 that controls the allocation of subcarriers to a subchannel in the sector of a cell. It implies the same subchannel, in two different sectors using the same frequency band but different PermBase, will comprise of different subcarriers. Therefore, the aim of the algorithm is to assign Permbase to all sectors in such a way so that adjacent cells, or those overlapping with each other, have different PermBase values in order to avoid interference. The $D L_{\text {PermBase }}$ is an integer ranging from 0 to 31 .

When any sector is activated in the network a list of overlapping/interfering cells is immediately created for it. Each cell within the list is checked for the assigned PermBase id, which are then excluded for the assignment to a current sector. Having reduced the list of possible PermBase values available for allocation the one with the lowest value is chosen. 


\subsubsection{Slot assignment and interference calculation}

The AFP algorithm in Stage 2 is implemented by considering any user for assignment to a base station resulting in the allocation of time/frequency resources and the evaluation of interference on these slots. This determines the modulation scheme for transmission and achievable bit-rate at the SINR level. An overview of the slot assignment procedure is described in the Algorithm 6.2

The process begins by checking which slots are available for assignment at the given sector. Thereafter, the amount of subcarriers colliding with slots assigned to interfering cells in the network is evaluated. When all slots are checked, the sorted list is created in order of ascending collisions. The slots with the least amount of collisions experience the least amount of interference. The next step is to calculate the SINR at each slot from this list, which provides the AMC scheme the available at the SINR level and therefore achievable bit-rate. Having calculated the bit-rate the slots get assigned to a user until his traffic demand is satisfied.

\subsubsection{Stage objectives and cost function weights}

Due to the fact that the cost of the network is not taken into account in Stage 2 as none of the sectors or sites get activated or de-activated, the main objective of this stage is to increase the overall served traffic. With fixed expenditures an increase in the service level directly increases the profitability of the network as well. However, an additional objective is introduced specifically for Stage 2, namely the assignment ratio of users. As it has already been mentioned, during the sequential sectorization of omni cells another goal is to reproduce the same shape of the cell found from Stage 1, or in other words to assign as many users given by Stage 1 as possible. This guarantees high correlation of the overall network results between the optimization stages.

The objectives and their associated weights in the objective function for Stage 2 are: 


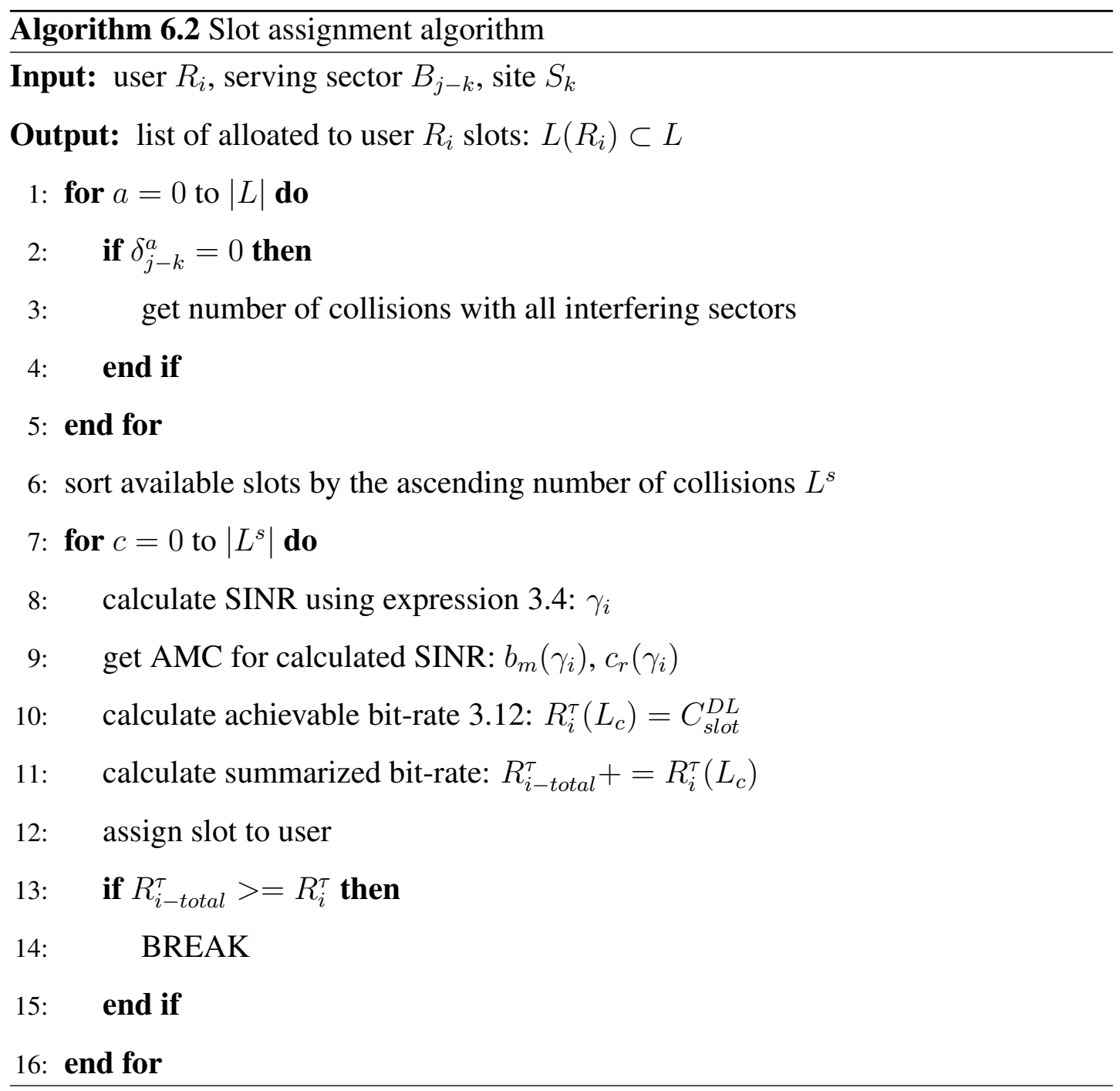

service maximization

0.5

assignment ratio maximization $\quad 0.5$

\subsubsection{Output of Stage 2}

The resulting output of Stage 2 is a selection of sites with fully sectorized base stations pre-configured for the final optimization stage. The comparison of network solutions produced by stage 1 and 2 can be seen in Figures 6.10 and 6.11 . Table 6.3 presents the comparison of results based on KPI measures. It can be seen that all performance 


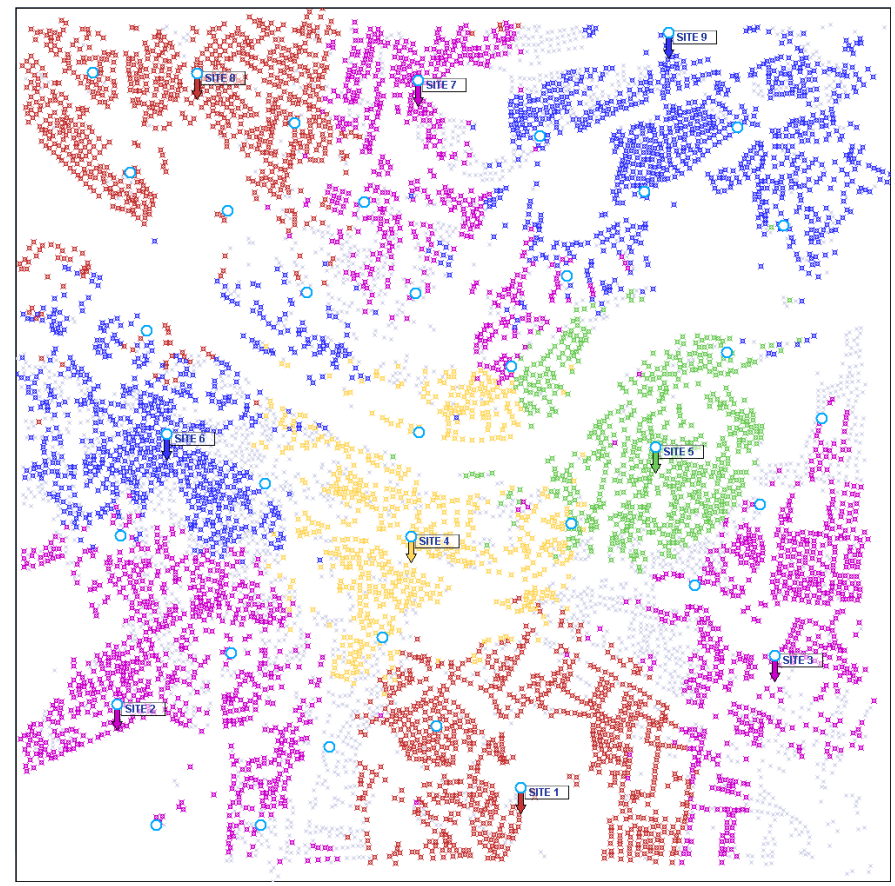

Figure 6.10: Network instance from the optimization Stage 1

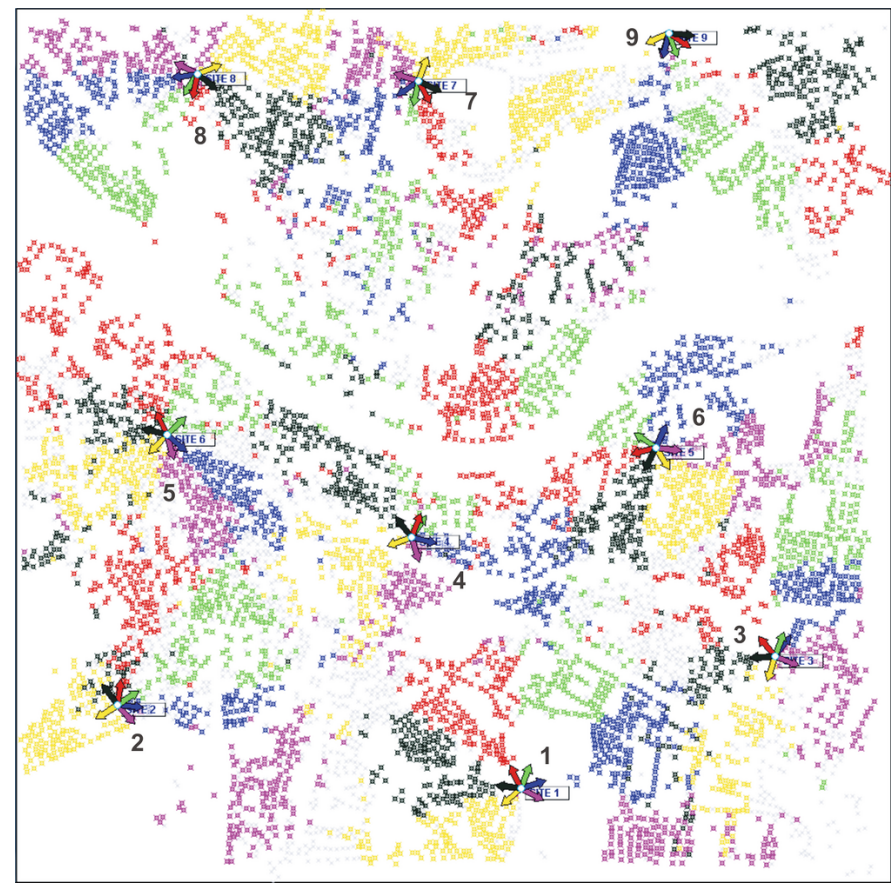

Figure 6.11: Network instance from the optimization Stage 2 
indicators of Stage 2 are close to the ones of Stage 1 with marginal improvement in performance. This is due to the fact that sectorization of omni cells and configuration of sectors is performed quickly to obtain the preliminary settings, which are not final or optimal in any sense.

Nevertheless, the sectorization (stage 2) produces results which are very close to the initial coverage (stage 1). This shows that all the assumptions related to capacity estimation and interference calculation for omni-directional base stations are suitable and tuned to produce results highly correlated to the real sectorized design. This can also be observed in Table 6.4 where the coverage and service levels are compared between a network with 9 cells in Stage 1 and 2. From this table it can be noted that sectorized cells tend to cover less users in order to service most effectively the pre-defined set of users given by Stage 1 and at the same time to reduce the potential interference within the network. Also the total amount of users covered by each sectorized cell is less than the sum of users covered by all sectors within the cell. This can be explained as some of the users are covered by several sectors at a site. The service level is approximately at the same level between stages. Also, it is noticeable that the central cells with indices ' 4', '5' and '7', being surrounded by the rest of the cells in the network, experience the highest level of interference which reduces the order of modulation and coding schemes used and therefore reducing the overall traffic load of the cells.

\begin{tabular}{|l|l|l|}
\hline Key performance indicators & Stage 1 & Stage 2 \\
\hline Coverage level (\%) & 98.28 & 97.68 \\
Service level (\%) & 72.46 & 72.49 \\
Network cost (€) & 432000 & 432000 \\
Profit (€) & 187200 & 187506 \\
Traffic unit cost (€/Kbps) & 0.3506 & 0.347 \\
Spectral efficiency (bps/Hz) & 1.15 & 1.15 \\
Capacity (Mbps) & 1243 & 1244.4 \\
\hline
\end{tabular}

Table 6.3: Key performance indicators comparison between Stage 1 and 2 


\begin{tabular}{|c|c|c|c|c|c|c|c|c|c|}
\hline \multirow{2}{*}{ KPI } & \multirow{2}{*}{ Site } & \multirow{2}{*}{ Stage 1} & \multicolumn{7}{|c|}{ Stage 2 sectors } \\
\hline & & & 1 & 2 & 3 & 4 & 5 & 6 & Total \\
\hline \multirow{9}{*}{ Coverage } & 1 & 4820 & 573 & 448 & 901 & 593 & 1085 & 905 & 2909 \\
\hline & 2 & 5397 & 126 & 961 & 280 & 892 & 533 & 776 & 2624 \\
\hline & 3 & 1915 & 243 & 108 & 158 & 372 & 194 & 312 & 895 \\
\hline & 4 & 4404 & 435 & 243 & 723 & 522 & 842 & 389 & 2091 \\
\hline & 5 & 4089 & 443 & 406 & 450 & 1302 & 523 & 217 & 2251 \\
\hline & 6 & 2669 & 314 & 250 & 322 & 1049 & 382 & 134 & 1800 \\
\hline & 7 & 3049 & 105 & 164 & 318 & 130 & 176 & 584 & 1063 \\
\hline & 8 & 3919 & 616 & 204 & 626 & 373 & 1896 & 577 & 2683 \\
\hline & 9 & 3858 & 909 & 3358 & 509 & 598 & 1071 & 220 & 3993 \\
\hline \multirow{9}{*}{ Service } & 1 & 754 & 134 & 118 & 143 & 110 & 146 & 127 & 778 \\
\hline & 2 & 713 & 117 & 147 & 66 & 160 & 139 & 71 & 700 \\
\hline & 3 & 638 & 110 & 98 & 97 & 136 & 107 & 86 & 634 \\
\hline & 4 & 626 & 127 & 86 & 142 & 63 & 116 & 134 & 668 \\
\hline & 5 & 622 & 137 & 122 & 123 & 121 & 108 & 110 & 721 \\
\hline & 6 & 687 & 155 & 105 & 116 & 139 & 127 & 75 & 717 \\
\hline & 7 & 505 & 92 & 88 & 104 & 78 & 94 & 84 & 540 \\
\hline & 8 & 826 & 146 & 120 & 123 & 117 & 102 & 146 & 754 \\
\hline & 9 & 848 & 144 & 74 & 142 & 138 & 86 & 131 & 715 \\
\hline
\end{tabular}

Table 6.4: Comparison of the number of covered and serviced users between Stage

\section{1 and 2.}

Results presented in Figures 6.12 and 6.13 reflect the sectorization progress of omnidirectional cells in terms of the cost function minimization score. Figure 6.12 compares cost function score over time for all cells. It can be seen that the application of the greedy heuristic finds a local minima within the first 500 iterations with some minor improvement for some cells. This approach guarantees quick discovery of a good solution which can be further improved in the final stage (Stage 3). Figure 6.13 
on the other hand shows the combined value of the cost function score value for the whole network during Stage 2, where the cells are sectorized in a sequential manner. Each peak in the graph appears when the new, initially sectorized cell, is activated in the network. Sectors configured with default settings do not efficiently use the capacity resources which reflects in the high solution cost. However, after a series of modifications the algorithm converges to a good solution with a low score for the cost function. Beginning with the first cell to be sectorized it can be seen that with an empty network and without any interferers present the variation between the initial score and the final is much greater than for the last cell, where the network is almost fully serviced and with a large amount of interference. This makes it difficult for the algorithm to find configurations for the sectors which improve the overall solution quality. Also it can be noted that the overall trend of the graph in a sense simulates the SA progression through the optimization process with acceptance of uphill moves and minimization of acceptance probability towards the end of the algorithm, which is reflected in the smaller hikes. 


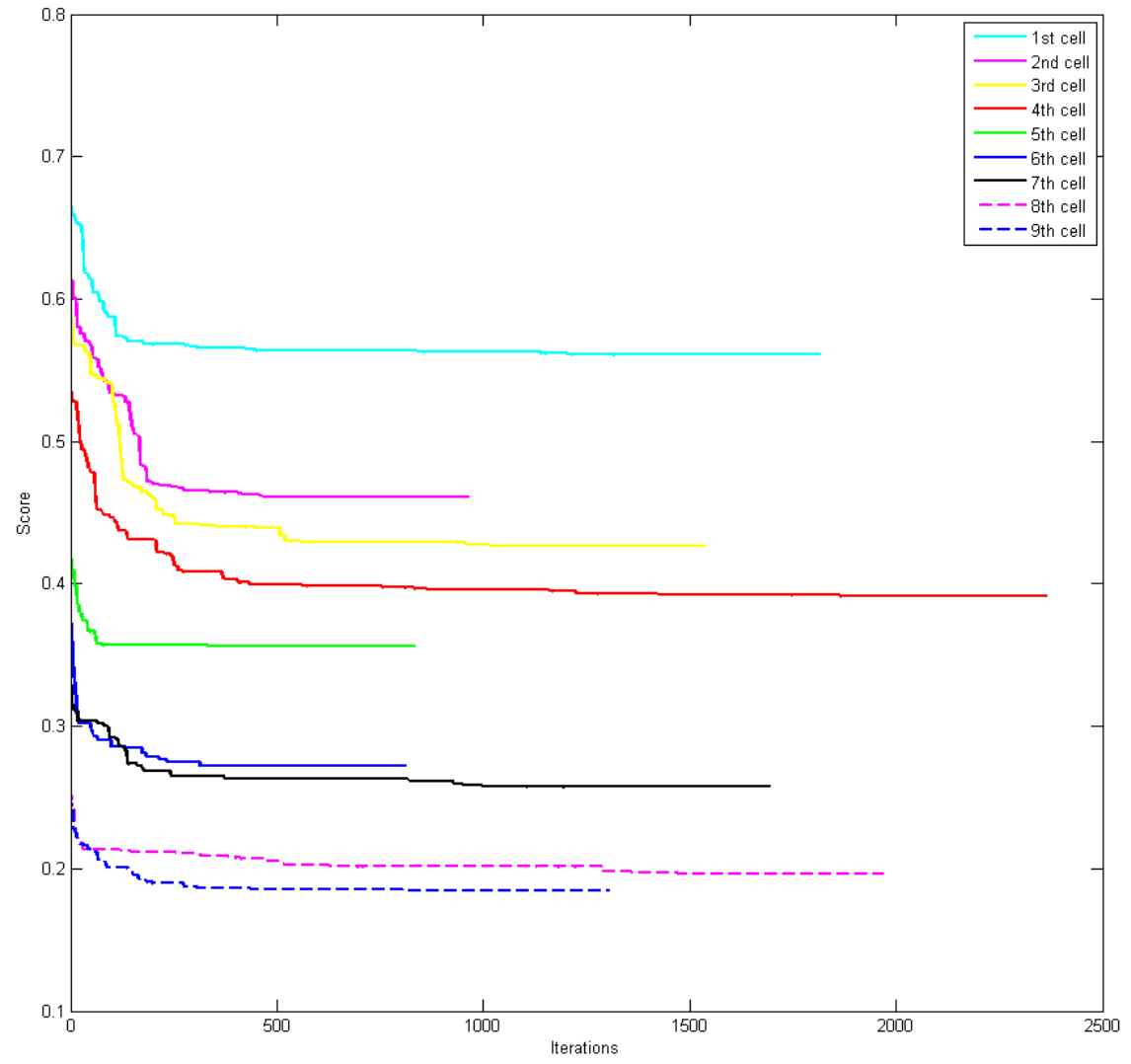

Figure 6.12: Cell-by-cell sectorization score results in Stage 2 


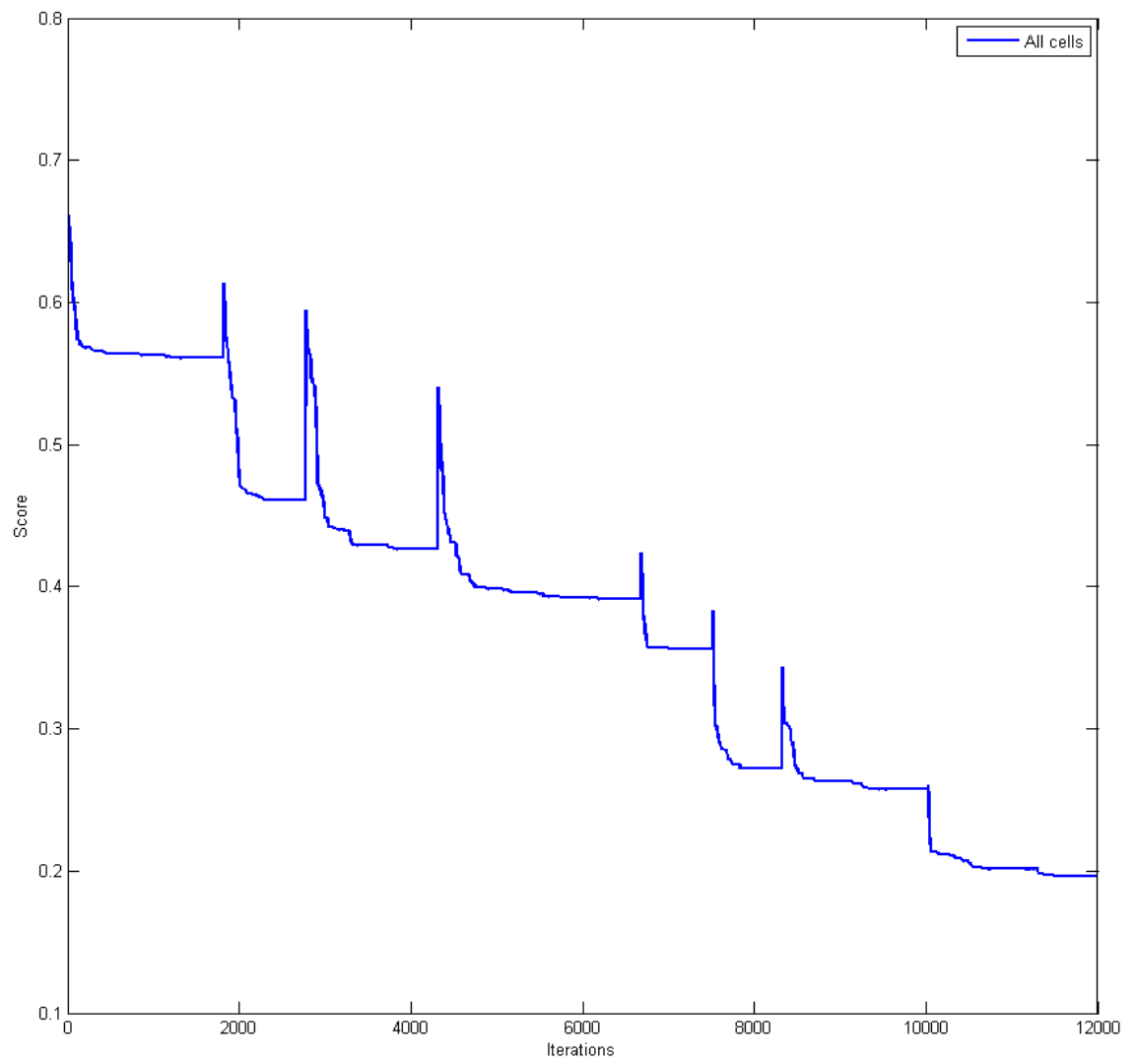

Figure 6.13: Overall score results in Stage 2 


\subsection{Stage 3. Final optimization}

After all sites have been sectorized and pre-configured in Stage 2, Stage 3 takes into account the full network instance for the final optimization where the main objective is to increase the network service level. The outline of the final stage is depicted in Figure 6.14. The set of neighbourhood moves used is the same as in Stage 2:

- change power

- change azimuth

- change tilt

- change antenna type

As with Stage 1 the algorithm used to optimize the given objectives for Stage 3 is simulated annealing. The initial temperature of the SA is set to a lower value compared to Stage 1 in order to reduce the likelihood of acceptance of large uphill (worse) moves. This measure is set to avoid significant alteration of a good solution obtained from Stage 2, as the main goal is to maintain approximately the same solution structure throughout the Stage 3. The stopping criteria is set to 700 iterations without improvement of the solution's cost function score. At each iteration the algorithm randomly selects an active site and a random sector for modification and according to the move selection mechanism (section 4.1.4) performs a re-configuration. After a set of perturbations has been executed the probabilities of moves are updated according to the acceptance ratios of moves and the subsequent set of iterations selects the modifiers with the new probabilities.

\subsubsection{Stage objectives and cost function weights}

Similarly to Stage 2 the financial component of the network is not affected in the final stage of optimization. The main objectives thus are set to maximize the overall 


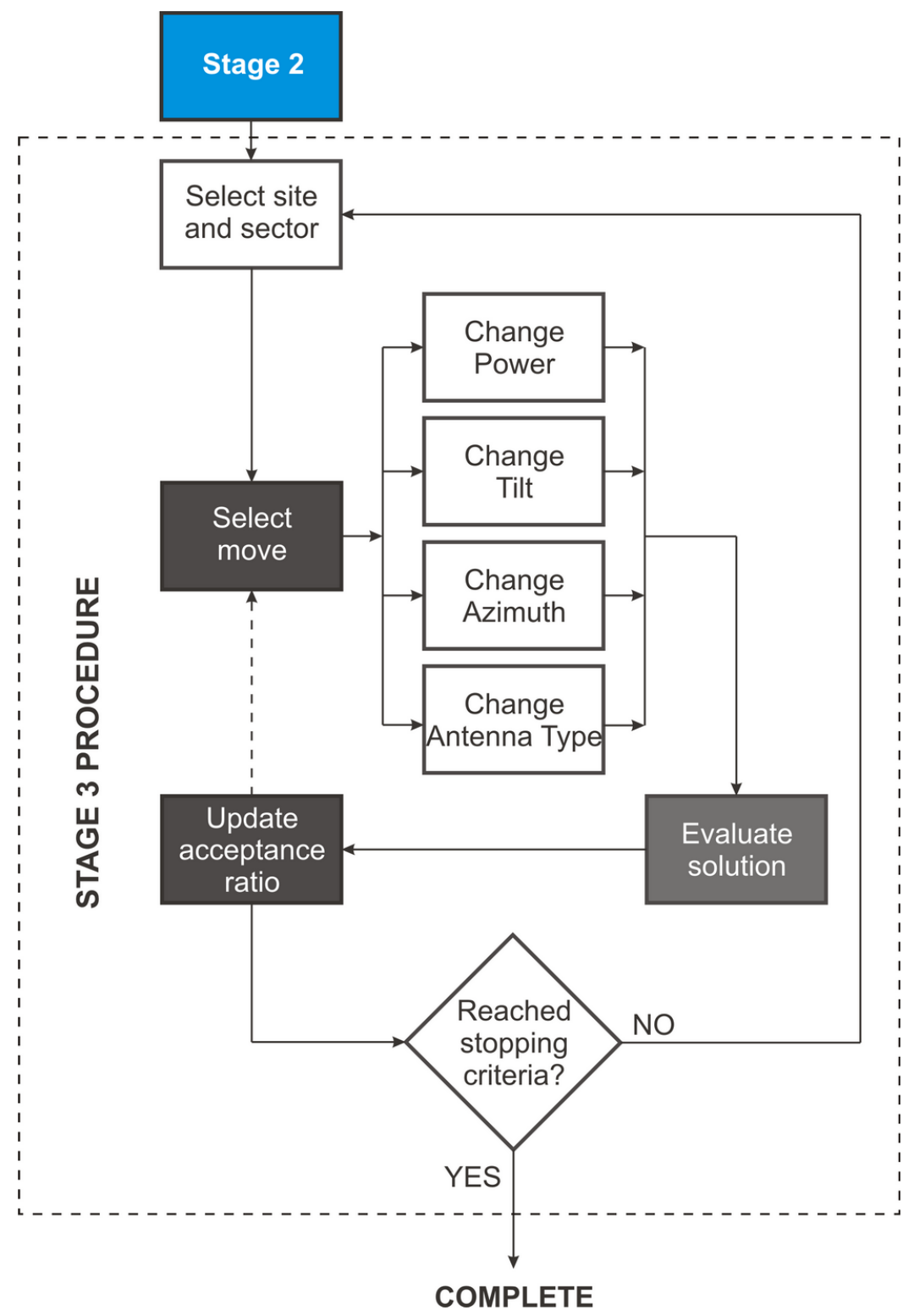

Figure 6.14: Stage 3 procedure

coverage and service levels with the major emphasis on the second objective.

The objectives and their associated weights in the objective function for Stage 3 are:

$\begin{array}{ll}\text { coverage maximization } & 0.2 \\ \text { service maximization } & 0.8\end{array}$ 


\subsubsection{Example output of Stage 3}

Stage 3 produces the final results of the fully sectorized and optimized WiMAX network. It consumes a considerable amount of computational time in comparison to the preceding stages as the amount of decision variables is higher. In addition the SA cooling schedule is tuned to reduce the temperature at a slower rate in order to let the system to settle into a lower energy state while sampling the solution space and to converge to a near optimal solution.

The network designs produced by stages 2 and 3 can be seen on Figures 6.15 and 6.16 respectively. Table 6.5 presents the comparison of key performance indicators for all stages. It can be seen that coverage of the network remains at about the same level at around $98 \%$. Although this objective is weighted much lower in comparison to other objectives throughout all the stages, having the service level as a priority guarantees good network coverage. The hypothetical service level of stage 1 is close to the real result of stage 2 and with the final stage improving this indicator by approximately $5 \%$. This increase in the service level is also reflected in the reduction of the traffic cost for the final solution. As it was mentioned in the section 6.1, the high correlation of results in terms of service level throughout the whole optimization framework underlines the fact that all the assumptions and techniques used in stage 1 for the evaluation of SINR and base station capacity are correctly defined. 


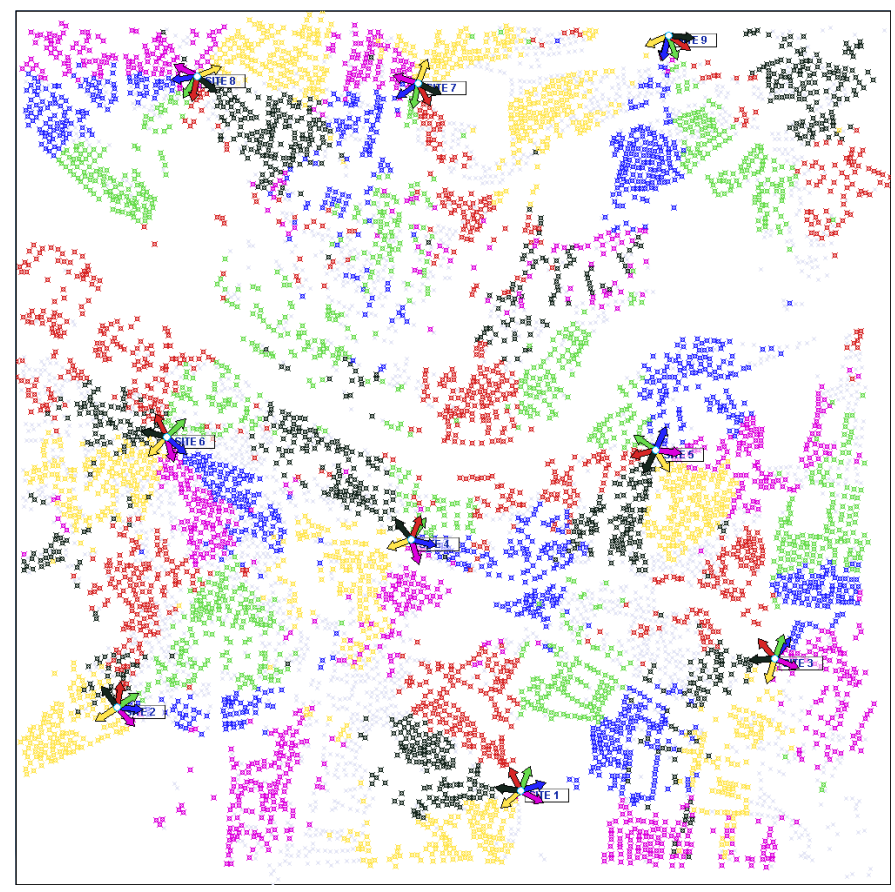

Figure 6.15: Network instance from the optimization Stage 2

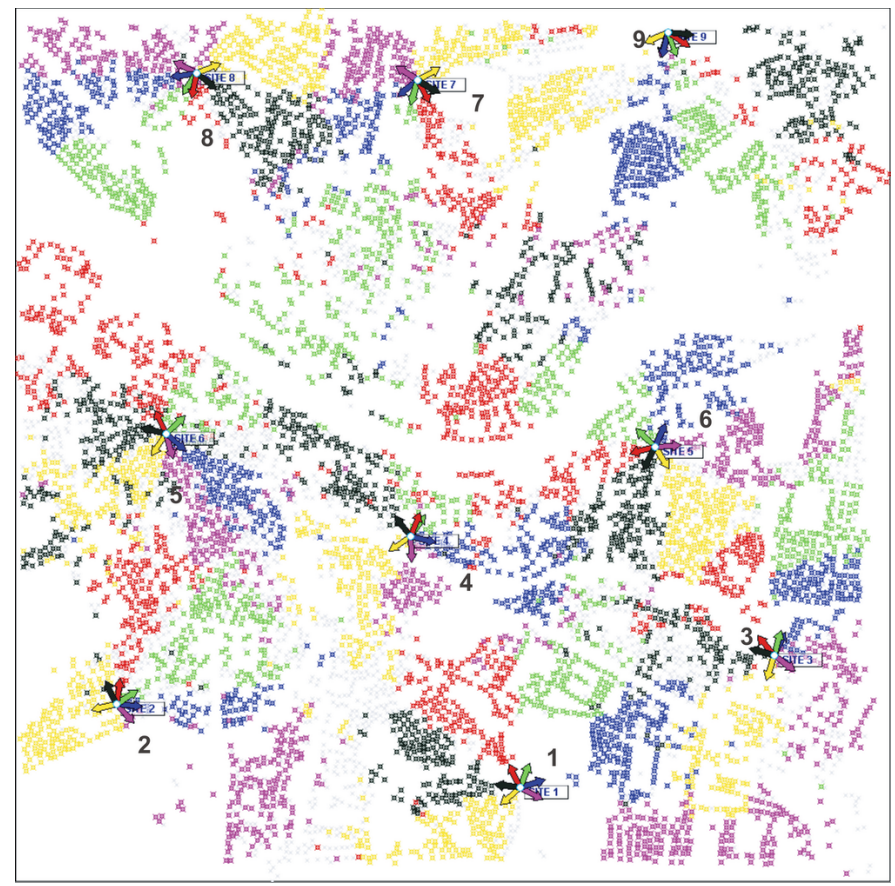

Figure 6.16: Comparison of resulting network plans from Stage 2 and 3 


\begin{tabular}{|l|c|c|c|}
\hline Key performance indicator & $\begin{array}{c}\text { Stage 1 } \\
\text { (dimensioning) }\end{array}$ & $\begin{array}{c}\text { Stage 2 } \\
\text { (sectorization) }\end{array}$ & $\begin{array}{c}\text { Stage 3 } \\
\text { (final) }\end{array}$ \\
\hline Coverage level (\%) & 98.28 & 97.68 & 97.96 \\
Service level (\%) & 72.46 & 72.49 & 75.66 \\
Network cost (€) & 432000 & 432000 & 432000 \\
Profit (€) & 187200 & 187506 & 214666 \\
Traffic unit cost (€/Kbps) & 0.3506 & 0.347 & 0.3326 \\
Spectral efficiency (bps/Hz) & 1.15 & 1.15 & 1.2 \\
Capacity (Mbps) & 1243 & 1244.4 & 1298.7 \\
\hline
\end{tabular}

Table 6.5: Key performance indicators comparison between Stage 1, 2 and 3

Tables $6.6 \& 6.7$ provide configuration settings for the sectorized base stations in the network solutions produced in stages 2 and 3. Almost all the sectors in the final network design have experienced at least one modification of antenna settings. 


\begin{tabular}{|c|c|c|c|c|c|c|c|c|c|c|}
\hline \multirow{2}{*}{ Sites } & \multirow{2}{*}{ Sectors } & \multicolumn{2}{|c|}{ Power (dBm) } & \multicolumn{2}{|c|}{ Azimuth } & \multicolumn{2}{|c|}{ Tilt } & \multicolumn{2}{|c|}{ Type } & \multirow{2}{*}{$\frac{\text { PB }}{\text { St2-3 }}$} \\
\hline & & St2 & St3 & St2 & St3 & St2 & $\mathrm{St3}$ & St2 & St3 & \\
\hline \multirow{6}{*}{1} & 1 & 43 & 43 & 311 & 311 & 10 & 10 & 30 & 30 & 0 \\
\hline & 2 & 31 & 35 & 62 & 62 & 8 & 7 & 30 & 30 & 8 \\
\hline & 3 & 37 & 37 & 108 & 108 & 10 & 10 & 30 & 30 & 10 \\
\hline & 4 & 35 & 35 & 159 & 159 & 11 & 11 & 30 & 30 & 27 \\
\hline & 5 & 41 & 41 & 206 & 206 & 11 & 11 & 30 & 30 & 2 \\
\hline & 6 & 39 & 39 & 263 & 263 & 11 & 11 & 30 & 30 & 18 \\
\hline \multirow{6}{*}{2} & 1 & 45 & 45 & 303 & 283 & 11 & 12 & 120 & 120 & 16 \\
\hline & 2 & 41 & 41 & 42 & 42 & 14 & 14 & 60 & 60 & 11 \\
\hline & 3 & 27 & 27 & 84 & 84 & 14 & 14 & 60 & 60 & 28 \\
\hline & 4 & 41 & 41 & 123 & 123 & 14 & 14 & 30 & 30 & 13 \\
\hline & 5 & 37 & 37 & 172 & 172 & 14 & 14 & 30 & 30 & 29 \\
\hline & 6 & 37 & 35 & 216 & 206 & 0 & 8 & 120 & 120 & 6 \\
\hline \multirow{6}{*}{3} & 1 & 37 & 45 & 343 & 343 & 10 & 10 & 60 & 30 & 6 \\
\hline & $2 \square$ & 43 & 45 & 68 & 53 & 11 & 11 & 60 & 90 & 1 \\
\hline & 3 & 39 & 43 & 144 & 154 & 12 & 12 & 30 & 30 & 17 \\
\hline & 4 & 41 & 41 & 160 & 165 & 6 & 6 & 30 & 30 & 13 \\
\hline & 5 & 39 & 41 & 220 & 220 & 10 & 10 & 60 & 60 & 14 \\
\hline & ${ }^{6}$ & 39 & 45 & 274 & 254 & 10 & 7 & 90 & 30 & 7 \\
\hline \multirow{6}{*}{4} & 1 & 39 & 43 & 290 & 305 & 10 & 11 & 60 & 60 & 12 \\
\hline & 2 & 35 & 35 & 17 & 2 & 12 & 12 & 60 & 60 & 4 \\
\hline & $3^{3}$ & 45 & 45 & 76 & 76 & 9 & 13 & 30 & 30 & 25 \\
\hline & 4 & 35 & 35 & 145 & 145 & 13 & 13 & 90 & 90 & 19 \\
\hline & 5 & 39 & 39 & 158 & 158 & 7 & 7 & 30 & 30 & 26 \\
\hline & 6 & 43 & 43 & 218 & 218 & 9 & 9 & 30 & 30 & 8 \\
\hline
\end{tabular}

Table 6.6: Re-configuration of sectors in Stage 3 (sites 1-4)

Finally Table 6.8 illustrates the comparison of the main KPI results between stages 2 and 3 on a sector basis. It can be noted that although some of the sectors have decreased in performance, most have increased, which satisfies the aim of the optimization framework - finding the balance between the settings which improves the overall performance. 


\begin{tabular}{|c|c|c|c|c|c|c|c|c|c|c|}
\hline \multirow{2}{*}{ Sites } & \multirow{2}{*}{ Sectors } & \multicolumn{2}{|c|}{ Power (dBm) } & \multicolumn{2}{|c|}{ Azimuth } & \multicolumn{2}{|c|}{ Tilt } & \multicolumn{2}{|c|}{ Type } & \multirow{2}{*}{$\frac{\text { PB }}{\text { St2-3 }}$} \\
\hline & & St2 & St3 & St2 & St3 & St2 & St3 & St2 & St3 & \\
\hline \multirow{6}{*}{5} & 1 & 43 & 45 & 32 & 32 & 10 & 5 & 60 & 30 & 6 \\
\hline & 2 & 43 & 45 & 83 & 93 & 4 & 5 & 30 & 30 & 3 \\
\hline & 3 & 39 & 41 & 156 & 156 & 12 & 12 & 60 & 60 & 0 \\
\hline & 4 & 45 & 43 & 236 & 226 & 11 & 13 & 60 & 60 & 24 \\
\hline & 5 & 43 & 41 & 285 & 285 & 7 & 7 & 30 & 30 & 9 \\
\hline & 6 & 43 & 45 & 333 & 333 & 9 & 9 & 30 & 30 & 18 \\
\hline \multirow{6}{*}{6} & 1 & 45 & 45 & 316 & 326 & 10 & 7 & 60 & 30 & 23 \\
\hline & 2 & 43 & 43 & 15 & 15 & 10 & 5 & 30 & 30 & 31 \\
\hline & 3 & 41 & 41 & 49 & 49 & 9 & 9 & 30 & 30 & 17 \\
\hline & 4 & 45 & 45 & 134 & 134 & 11 & 11 & 60 & 60 & 30 \\
\hline & 5 & 41 & 41 & 205 & 205 & 11 & 11 & 60 & 60 & 29 \\
\hline & 6 & 35 & 35 & 256 & 256 & 14 & 14 & 60 & 60 & 8 \\
\hline \multirow{6}{*}{7} & 1 & 45 & 45 & 159 & 119 & 10 & 4 & 90 & 90 & 29 \\
\hline & 2 & 39 & 45 & 252 & 237 & 10 & 13 & 90 & 60 & 21 \\
\hline & 3 & 41 & 39 & 304 & 309 & 10 & 10 & 60 & 60 & 7 \\
\hline & 4 & 37 & 45 & 349 & 344 & 10 & 7 & 30 & 30 & 11 \\
\hline & 5 & 43 & 45 & 28 & 28 & 11 & 12 & 30 & 30 & 22 \\
\hline & 6 & 45 & 45 & 64 & 59 & 9 & 7 & 90 & 90 & 23 \\
\hline \multirow{6}{*}{8} & 1 & 41 & 41 & 112 & 112 & 14 & 14 & 30 & 30 & 31 \\
\hline & 2 & 45 & 45 & 243 & 243 & 14 & 14 & 30 & 30 & 20 \\
\hline & 3 & 39 & 39 & 278 & 278 & 11 & 11 & 30 & 30 & 2 \\
\hline & 4 & 37 & 37 & 327 & 327 & 14 & 14 & 30 & 30 & 15 \\
\hline & 5 & 37 & 37 & 348 & 348 & 11 & 11 & 90 & 90 & 5 \\
\hline & ${ }^{6}$ & 37 & 37 & 51 & 51 & 14 & 14 & 30 & 30 & 3 \\
\hline \multirow{6}{*}{9} & 1 & 45 & 45 & 294 & 294 & 10 & 10 & 30 & 30 & 16 \\
\hline & 2 & 37 & 37 & 342 & 342 & 3 & 3 & 120 & 120 & 20 \\
\hline & 3 & 37 & 37 & 346 & 346 & 11 & 11 & 30 & 30 & 21 \\
\hline & 4 & 35 & 35 & 19 & 19 & 10 & 10 & 30 & 30 & 1 \\
\hline & 5 & 37 & 37 & 55 & 55 & 2 & 2 & 60 & 60 & 12 \\
\hline & 6 & 37 & 43 & 84 & 84 & 10 & 10 & 30 & 30 & 9 \\
\hline
\end{tabular}

Table 6.7: Re-configuration of sectors in Stage 3 (sites 5-9) 


\begin{tabular}{|c|c|c|c|c|c|c|c|c|c|c|c|c|c|}
\hline \multirow{3}{*}{ KPI } & \multirow{3}{*}{ Cell } & \multicolumn{12}{|c|}{ Sectors } \\
\hline & & \multicolumn{2}{|c|}{1} & \multicolumn{2}{|c|}{2} & \multicolumn{2}{|c|}{3} & \multicolumn{2}{|c|}{4} & \multicolumn{2}{|c|}{5} & \multicolumn{2}{|c|}{6} \\
\hline & & St2 & St3 & St2 & St3 & St2 & St3 & St2 & St3 & St2 & St3 & St2 & St3 \\
\hline \multirow{9}{*}{ Coverage } & 1 & 573 & 573 & 448 & 304 & 901 & 901 & 593 & 593 & 1085 & 1085 & 905 & 905 \\
\hline & 2 & 126 & 135 & 961 & 961 & 280 & 280 & 892 & 892 & 533 & 533 & 776 & 536 \\
\hline & 3 & 243 & 185 & 108 & 160 & 158 & 174 & 372 & 317 & 194 & 146 & 312 & 185 \\
\hline & 4 & 435 & 475 & 243 & 135 & 723 & 408 & 522 & 522 & 842 & 842 & 389 & 389 \\
\hline & 5 & 443 & 350 & 406 & 450 & 450 & 525 & 1302 & 752 & 523 & 401 & 217 & 249 \\
\hline & 6 & 314 & 242 & 250 & 240 & 322 & 322 & 1049 & 1049 & 382 & 382 & 134 & 134 \\
\hline & 7 & 105 & 129 & 164 & 155 & 318 & 147 & 130 & 335 & 176 & 133 & 584 & 797 \\
\hline & 8 & 616 & 616 & 204 & 204 & 626 & 626 & 373 & 373 & 1896 & 1896 & 577 & 577 \\
\hline & 9 & 909 & 909 & 3358 & 3358 & 509 & 509 & 598 & 598 & 1071 & 1071 & 220 & 320 \\
\hline \multirow{9}{*}{ Service } & 1 & 134 & 131 & 118 & 153 & 143 & 152 & 110 & 107 & 146 & 147 & 127 & 130 \\
\hline & 2 & 117 & 128 & 147 & 149 & 66 & 64 & 160 & 156 & 139 & 136 & 71 & 98 \\
\hline & 3 & 110 & 119 & 98 & 110 & 97 & 116 & 136 & 130 & 107 & 91 & 86 & 95 \\
\hline & 4 & 127 & 142 & 86 & 79 & 142 & 141 & 63 & 65 & 116 & 124 & 134 & 136 \\
\hline & 5 & 137 & 158 & 122 & 143 & 123 & 121 & 121 & 96 & 108 & 116 & 110 & 129 \\
\hline & 6 & 155 & 142 & 105 & 112 & 116 & 119 & 139 & 140 & 127 & 133 & 75 & 93 \\
\hline & 7 & 92 & 95 & 88 & 118 & 104 & 82 & 78 & 131 & 94 & 98 & 84 & 103 \\
\hline & 8 & 146 & 145 & 120 & 122 & 123 & 123 & 117 & 117 & 102 & 106 & 146 & 146 \\
\hline & 9 & 144 & 143 & 74 & 78 & 142 & 143 & 138 & 134 & 86 & 71 & 131 & 141 \\
\hline
\end{tabular}

Table 6.8: Comparison of the number of covered and serviced users between Stages 2 and 3. 
Chapter 7

\section{Results \& Discussion}

Adopting a novel approach to network design of practical scenarios this chapter presents the results and discusses the benefits in using the proposed three stage framework presented in Chapter 5 and Chapter 6. Several scenarios are considered for comparison with the proposed framework. First, the effect of PermBase assignment is investigated showing the deviation in KPIs when all the base stations in the network operate with the same PermBase. Next, we determine if the sectorization process proposed in Section 6.1 provides any advantage to the network optimization strategy. Further we explore the interference model proposed for Stage 1 and investigate the effect of varying the parameters in the SINR. Finally we build the network employing the standard approach widely presented in the literature in order to compare the results on a simplified problem formulation.

The structure of this chapter is as follows: Section 7.1 presents the network data and a summary of all the network configurations used in this thesis for WiMAX network planning evaluation. Section 7.2 provides the results of the three stage framework proposed in this thesis. Section 7.3 evaluates the advantage of the PermBase assignment process. Section 7.4 assesses the benefits of the Stage 2 sectorization procedure. Section 7.5 evaluates the interference model of Stage 1. Section 7.6 provides the results for the standard network planning approach and finally Section 7.7 summarizes the results of all the scenarios with full analysis and conclusions. 


\subsection{Experimental Network Scenario}

The experimental network was obtained from the EMBRACE (Efficient Millimetre Broadband Radio Access for Convergence and Evolution) project [2]. The main purpose of this project was to investigate and develop efficient and low cost radio access systems for a variety of traffic load scenarios including urban, suburban and rural areas in order to provide users with a full range of broadband services such as digital television, video on demand, high speed internet access and voice communications. The way to achieve this was through efficient utilisation of radio frequency bands and optimisation of transmission capacities for a variety of users and usage.

To predict radio wave propagation in dense urban environments the planning tool RPD (Rapid Pipeline Development) was developed which takes into account obstructions from the topography and man-made structures, the effects of atmospheric propagation conditions and the antenna radiation patterns. This tool is described in Section 3.2

The scenario used for the experiments in thesis is based on the city of Newcastle, UK. The data explicitly defining the network is structured as follows:

1. RTP table - IDs, $x-y-z$ coordinates of reception test points, traffic demand in Kbps and annual revenue which can be generated from the users. The sample of data is shown in Table 7.1

2. Site locations table - site id, $x-y-z$ coordinates, costs for installation and annual maintenance. The sample of data is shown in Table 7.2

3. Losses table - pathloss figures for each base station / receiver pair in $\mathrm{dBm}$. The sample of data is shown in Table 7.3

The graphical illustration of the network is depicted in Figure 7.1. The area of the region is approximately $17.69 \mathrm{~km}^{2}$. Each grey pixel in the figure represents an RTP and golden circles are site locations. There are 8583 RTP test points in total distributed 


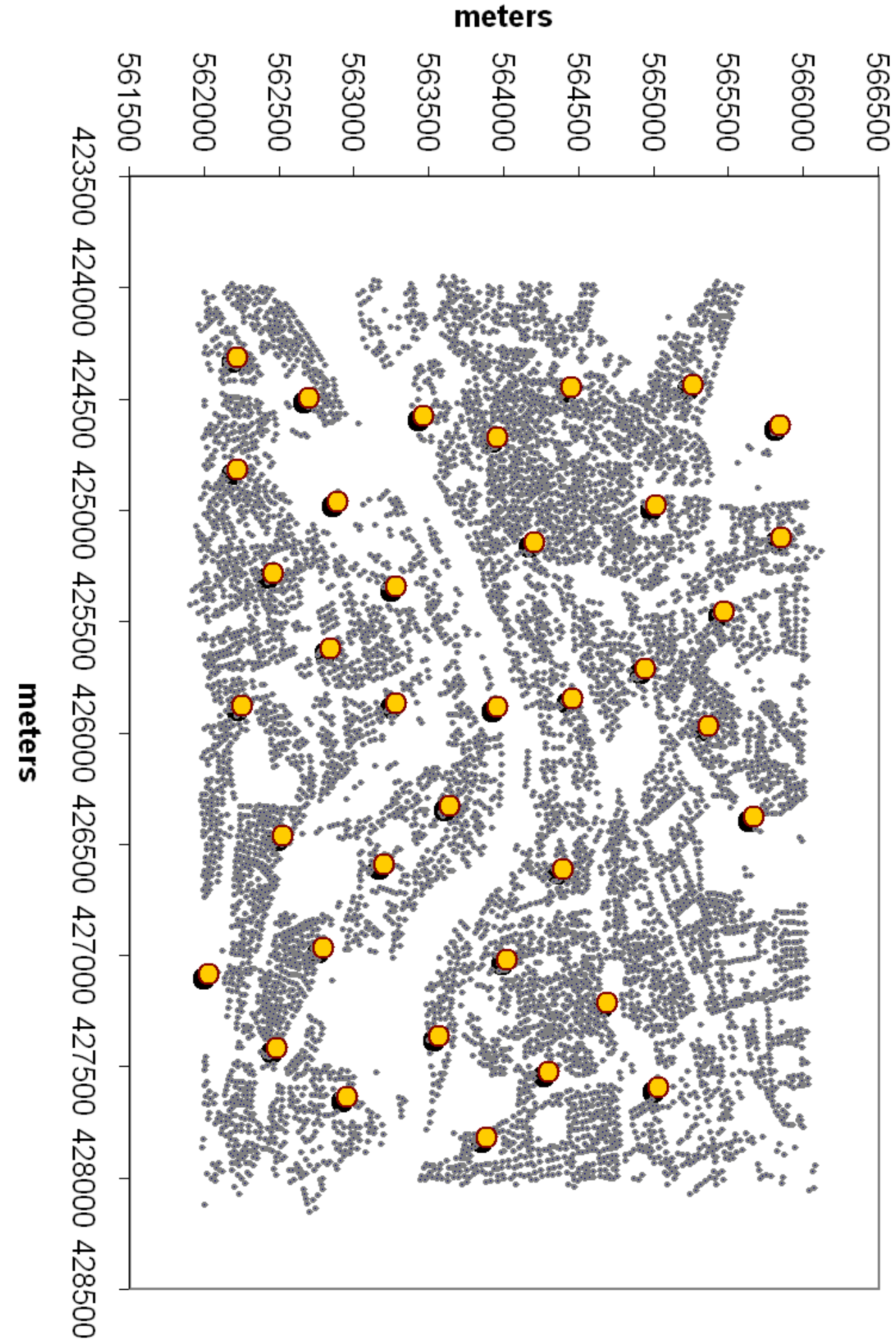

Figure 7.1: Newcastle city 


\begin{tabular}{lcccc}
\hline RTP ID & $\mathbf{X}(\mathbf{m})$ & $\mathbf{Y}(\mathbf{m})$ & $\mathbf{Z}(\mathbf{m})$ & Rate $(\mathbf{K b p s})$ \\
\hline 42 & 426221 & 562034 & 111.25 & 200 \\
43 & 426206 & 562051 & 111 & 200 \\
44 & 426105 & 562052 & 111 & 200 \\
45 & 426212 & 562016 & 111 & 200 \\
46 & 426659 & 562018 & 110.75 & 200 \\
47 & 426735 & 562000 & 110.5 & 200 \\
48 & 426499 & 562053 & 110.5 & 200 \\
\hline
\end{tabular}

Table 7.1: Sample of RTPs data

\begin{tabular}{lccccc}
\hline Site ID & $\mathbf{X}(\mathbf{m})$ & $\mathbf{Y}(\mathbf{m})$ & $\mathbf{Z}(\mathbf{m})$ & CAPEX $(€)$ & OPEX $(€)$ \\
\hline 5 & 425969 & 565365 & 57 & 30000 & 1500 \\
6 & 424436 & 565260 & 137 & 30000 & 1500 \\
7 & 427594 & 565024 & 67 & 30000 & 1500 \\
8 & 424981 & 565012 & 116 & 30000 & 1500 \\
9 & 425711 & 564936 & 116 & 30000 & 1500 \\
10 & 427212 & 564688 & 91 & 30000 & 1500 \\
11 & 425846 & 564450 & 64 & 30000 & 1500 \\
\hline
\end{tabular}

Table 7.2: Sample of site locations data

across the city each of them representing a user with geographical position defined and 37 sites. The traffic demand and service requirements are uniformly defined for all subscribers with $200 \mathrm{Kbps}$. Therefore, it is assumed that the revenue generated from the served user is the same and is fixed at a level of 100 euros per user for a period of one year. The propagation figures used are designed for a $3.5 \mathrm{GHz}$ radio access system. Based on the network model presented in Chapter 3 table 7.4 summarizes the main 


\begin{tabular}{lcl}
\hline Site ID & RTP ID & Loss $(\mathbf{d B m})$ \\
\hline 1 & 542 & 113.189 \\
1 & 543 & 146.367 \\
1 & 544 & 137.27 \\
1 & 545 & 114.812 \\
1 & 546 & 129.144 \\
1 & 547 & 103.862 \\
1 & 548 & 135.727 \\
\hline
\end{tabular}

Table 7.3: Sample of path-loss figures

parameters used for the downlink network planning. 


\begin{tabular}{|c|c|}
\hline PARAMETERS & VALUES \\
\hline Frequency band & $3.5 \mathrm{GHz}$ \\
\hline Channel bandwidth & $20 \mathrm{MHz}$ \\
\hline Frequency reuse pattern & $1 \times 1$ \\
\hline Max number of sectors per BS & 6 \\
\hline Maximum EIRP per sector & $45 \mathrm{dBm}$ \\
\hline Tx antenna gain - 30 degrees pattern & $22.9 \mathrm{dBi}$ \\
\hline Tx antenna gain - 60 degrees pattern & $17.5 \mathrm{dBi}$ \\
\hline Tx antenna gain - 90 degrees pattern & $16 \mathrm{dBi}$ \\
\hline Tx antenna gain - 120 degrees pattern & $15 \mathrm{dBi}$ \\
\hline User antenna gain & $0 \mathrm{dBi}$ \\
\hline Permutation mode & PUSC \\
\hline PermBase & $0-31$ \\
\hline Frame duration & $5 \mathrm{~ms}$ \\
\hline DL symbols & 37 \\
\hline DL usable symbols & 28 \\
\hline Subchannels per symbol & 60 \\
\hline Slot structure & 1 subchannel $\times 2$ symbols \\
\hline DL subframe capacity & 840 slots \\
\hline Duplexing & TDD \\
\hline Rate per user & $200 \mathrm{Kbps}$ \\
\hline Modulation and coding & All modes, Table 3.7 \\
\hline Site installation costs - CAPEX & $€ 30000$ \\
\hline Site maintenance costs (per annum) - OPEX & $€ 1500$ \\
\hline Sector equipment cost - CAPEX & $€ 1500$ \\
\hline User generated revenue (per annum) & $€ 100$ \\
\hline
\end{tabular}

Table 7.4: Network main parameters 


\subsection{Framework results}

This section presents results for the three stage framework proposed in this thesis. A number of trials were performed for each stage of the optimization framework in order to show their effectiveness, correlation of the network plans between stages and the marginal improvements of KPIs in the final network planning stage. First, 25 cell plans were produced in Stage 1. All the network settings and objective function weights were fixed for all tests. Variation in the runs was achieved by the different starting points in the search space and the seeds of the random number generator. The performance of the resulting network plans can be seen in Tables 7.5 and 7.6 ('score' denotes overall objective function value). The best performing designs in one of the KPIs are marked in green and the worst in red. It can be noted that most of the cell plans produced in this stage were constructed of 9 base stations with the maximum number of deployable sectors at each site. Satisfying a coverage level in the network is an important criteria for service providers and most of the networks have achieved a level above $95 \%$. With regard to service, the maximum level of $72.46 \%$ produced by network plan s1-10 indicates that the WiMAX system employing the frequency reuse scheme of 1 is interference limited (meaning that without the introduction of an additional channel the service level cannot be improved to achieve the full service). Since most of the plans having the same amount of equipment deployed in the networks, i.e. same financial commitment, the profitability depends on the number of users serviced, making network plan s1-10 the most profitable. An interesting observation can be noted with regard to the relation between the traffic unit cost and spectral efficiency - the network plan with the worst use of spectrum (s1-20) produces the highest cost of the traffic unit, and conversely for (s1-25).

The distribution of the results, over the 25 trials, in terms of the objective function cost is as follows: 


$\begin{array}{lr}\text { Maximum } & 0.3032 \\ \text { Mean } & 0.2754 \\ \text { Minimum } & 0.2596 \\ \text { Deviation } & 0.0108 \\ \text { CV } & 3.9 \%\end{array}$

With the coefficient of variation (CV) standing at only $3.9 \%$ it can be said that the networks produced are very similar with minor differences in site selections and BS configurations. Five network designs were selected based on their cost function value for subsequent modifications. These networks are denoted as follows (Table 7.7):

- s1-max - worst result

- s1-dev(+) - half of the deviation above the mean value

- s1-mean - mean result

- s1-dev(-) - half of the deviation below the mean value

- s1-min - best result

The graphical representations of the selected network plans can be seen in Figures 7.2 -7.6 . 


\begin{tabular}{|c|c|c|c|c|c|c|c|c|c|c|c|c|c|c|c|c|}
\hline מ̆ & & $\begin{array}{l}\stackrel{0}{0} \\
\text { గ̦ }\end{array}$ & $\begin{array}{l}1 \\
\text { రิ } \\
0 \\
0\end{array}$ & 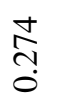 & $\begin{array}{l}\text { ָै } \\
\text { ஸे }\end{array}$ & 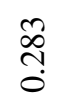 & $\hat{\hat{o}}$ & 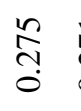 & 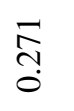 & $\underset{\widehat{ก}}{0}$ & त्रิ & 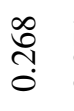 & $\underset{\hat{N}}{0}$ & 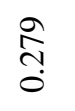 & $\begin{array}{l}\infty \\
\stackrel{0}{0} \\
i \\
0\end{array}$ & 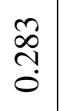 \\
\hline & 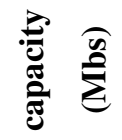 & $\stackrel{\stackrel{+}{ \pm}}{\stackrel{\Xi}{\Xi}}$ & $\stackrel{\infty}{\infty}$ & $\begin{array}{l}\infty \\
\stackrel{\infty}{a} \\
\stackrel{\beth}{\beth}\end{array}$ & $\begin{array}{l}\stackrel{+}{\infty} \\
\stackrel{\infty}{\Xi} \\
\end{array}$ & $\begin{array}{l}\infty \\
\stackrel{0}{0} \\
\end{array}$ & $\begin{array}{l}\infty \\
\ddot{j} \\
\text { ป }\end{array}$ & $\begin{array}{l}\stackrel{\nabla}{\bullet} \\
\stackrel{\Xi}{\Xi}\end{array}$ & $\begin{array}{l}\stackrel{0}{\mathrm{~d}} \\
\text { ป }\end{array}$ & 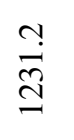 & $\begin{array}{l}\infty \\
\stackrel{\leftrightarrow}{d} \\
\end{array}$ & 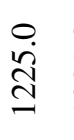 & 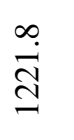 & $\begin{array}{l}\infty \\
\stackrel{0}{0} \\
\stackrel{0}{\beth}\end{array}$ & 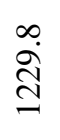 & $\begin{array}{l}\stackrel{1}{\infty} \\
\stackrel{\infty}{=} \\
=\end{array}$ \\
\hline & 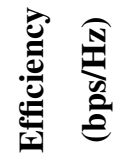 & $\stackrel{\Xi}{\Xi}$ & $\Xi$ & $\stackrel{\cong}{=}$ & $\stackrel{n}{=}$ & $\exists$ & $\stackrel{ \pm}{ \pm}$ & $\stackrel{m}{=}$ & $\stackrel{m}{=}$ & $\underset{:}{ \pm}$ & $\stackrel{\sim}{=}$ & $\stackrel{0}{=}$ & $\stackrel{m}{=}$ & $\stackrel{\simeq}{\leftrightarrows}$ & $\stackrel{\Xi}{\Xi}$ & $\exists$ \\
\hline 总 & 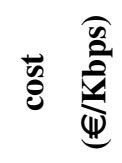 & 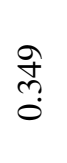 & $\stackrel{m}{\tilde{m}}$ & $\begin{array}{l}n \\
\tilde{n} \\
0\end{array}$ & 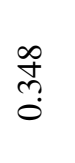 & ڤn & $\begin{array}{l}\tilde{n} \\
\tilde{n} \\
0\end{array}$ & $\begin{array}{l}n \\
\tilde{n} \\
0 \\
0\end{array}$ & \begin{tabular}{l}
\multirow{2}{n}{} \\
$\tilde{\sigma}$
\end{tabular} & $\ddot{n}$ & 缹 & $\ddot{n}$ & $\stackrel{n}{\tilde{n}}$ & 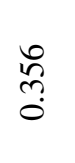 & ڤ్n & $\begin{array}{l}0 \\
\stackrel{?}{0}\end{array}$ \\
\hline $\overrightarrow{\bar{\varpi}}$ & $\begin{array}{l}\mathbb{\Psi} \\
\stackrel{E}{0} \\
\vdots \\
\vdots\end{array}$ & $\begin{array}{l}8 \\
0 \\
n \\
\infty \\
0\end{array}$ & 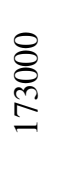 & $\begin{array}{l}\stackrel{N}{N} \\
\stackrel{ }{=}\end{array}$ & $\begin{array}{l}8 \\
0 \\
10 \\
\infty \\
0\end{array}$ & $\begin{array}{l}8 \\
8 \\
6 \\
6\end{array}$ & 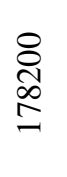 & 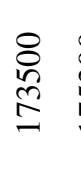 & $\begin{array}{l}8 \\
0 \\
n \\
\\
=\end{array}$ & $\begin{array}{l}8 \\
8 \\
8 \\
\infty\end{array}$ & $\underset{\substack{\hat{~} \\
\infty}}{\infty}$ & $\begin{array}{l}8 \\
\infty \\
\infty \\
\infty \\
\infty\end{array}$ & $\begin{array}{l}\text { ठิ } \\
\text { ปู }\end{array}$ & 分 & 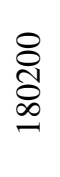 & 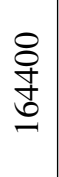 \\
\hline 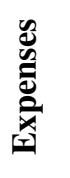 & $\mathbb{\Psi}$ & 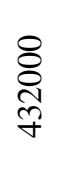 & $\begin{array}{l}\text { 高 } \\
\text { 商 }\end{array}$ & 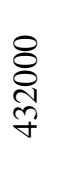 & 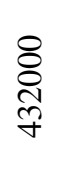 & 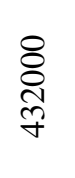 & $\begin{array}{l}\stackrel{8}{8} \\
\stackrel{\pi}{+}\end{array}$ & 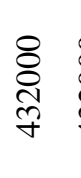 & 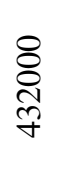 & 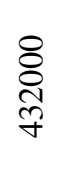 & 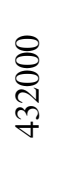 & $\begin{array}{l}\text { § } \\
\text { ণे } \\
\text { ণे }\end{array}$ & 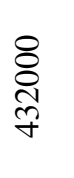 & 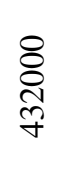 & 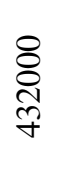 & 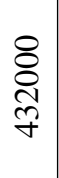 \\
\hline : & of & $\stackrel{\bar{a}}{\vec{\lambda}}$ & $\frac{\infty}{b}$ & $\begin{array}{l}\infty \\
\infty \\
\stackrel{\infty}{\infty}\end{array}$ & $\underset{i}{\stackrel{\Xi}{i}}$ & $\begin{array}{l}\text { ă } \\
\text { ğ }\end{array}$ & $\underset{\dot{i}}{\vec{i}}$ & $\begin{array}{l}\infty \\
\infty \\
\stackrel{R}{2}\end{array}$ & $\stackrel{5}{i}$ & $\stackrel{\mathbb{2}}{\stackrel{i}{i}}$ & 离 & 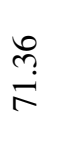 & $\stackrel{\infty}{\stackrel{\infty}{R}}$ & $\stackrel{n}{n}$ & & $\begin{array}{l}\infty \\
\ddot{b}\end{array}$ \\
\hline 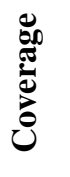 & $\widehat{\theta}$ & ?̊. & $\begin{array}{l}\stackrel{ָ}{a} \\
\stackrel{a}{a}\end{array}$ & $\begin{array}{l}0 \\
+ \\
\infty \\
\infty\end{array}$ & $\begin{array}{l}\stackrel{m}{\infty} \\
\infty \\
\infty\end{array}$ & 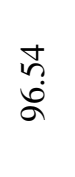 & $\begin{array}{l}\text { бे } \\
\text { ळे }\end{array}$ & $\begin{array}{l}\text { సิ } \\
\text { ๙ }\end{array}$ & $\begin{array}{l}\text { l) } \\
\infty \\
\infty\end{array}$ & $\stackrel{10}{\circ}$ & 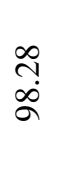 & $\frac{n}{\infty}$ & 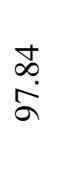 & $\vec{r}$ & $\begin{array}{l}\text { d̦ } \\
\text { s. }\end{array}$ & $\begin{array}{l}\infty \\
\infty \\
\\
a\end{array}$ \\
\hline 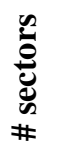 & & in & $\stackrel{\infty}{+}$ & in & in & it & in & in & in & in & in & $n$ & in & in & in & in \\
\hline $\begin{array}{l}\stackrel{0}{\tilde{c}} \\
\# \\
\#\end{array}$ & & $a$ & $\infty$ & $a$ & $a$ & $a$ & $a$ & $a$ & $a$ & $a$ & $a$ & $a$ & $a$ & $a$ & $a$ & $a$ \\
\hline 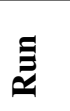 & & $\frac{T}{n}$ & $\frac{N}{n}$ & $\frac{n}{n}$ & $\frac{t}{\infty}$ & $\frac{n}{n}$ & $\frac{i}{i}$ & $\frac{i}{\infty}$ & $\frac{\infty}{\infty}$ & $\frac{a}{n}$ & $\frac{0}{\frac{1}{n}}$ & $\frac{7}{n}$ & $\frac{1}{\frac{1}{n}}$ & $\frac{m}{\frac{1}{n}}$ & $\frac{ \pm}{\infty}$ & $\frac{n}{\frac{1}{n}}$ \\
\hline
\end{tabular}




\begin{tabular}{|c|c|}
\hline 嗙 & 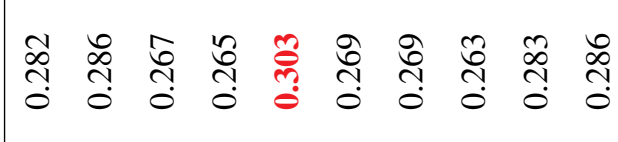 \\
\hline 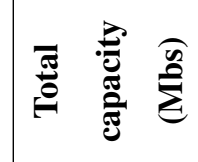 & 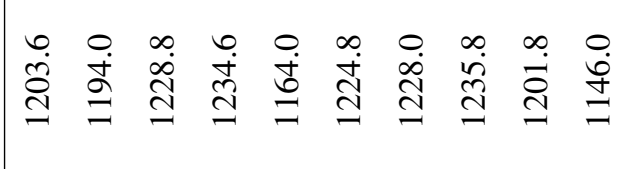 \\
\hline & 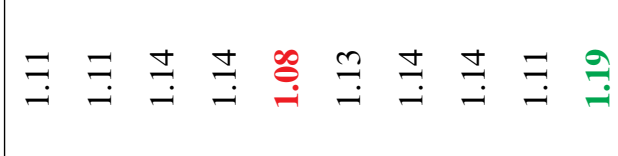 \\
\hline 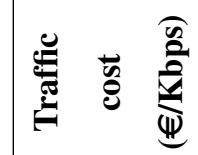 & 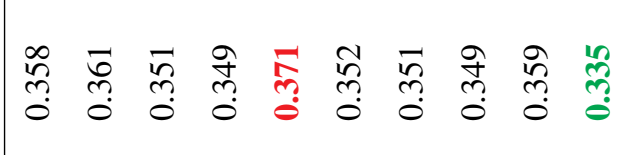 \\
\hline 氞 & 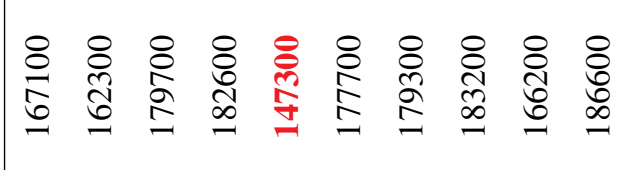 \\
\hline 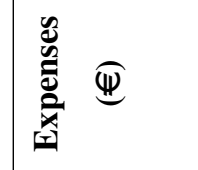 & 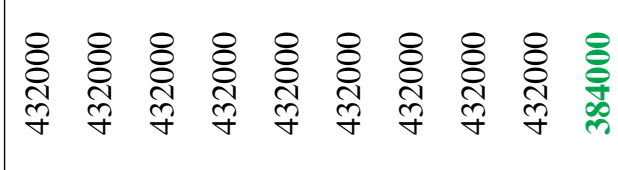 \\
\hline 赵 & 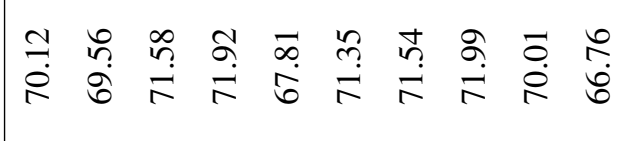 \\
\hline 常 & 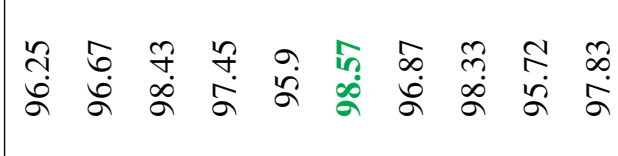 \\
\hline 童 & 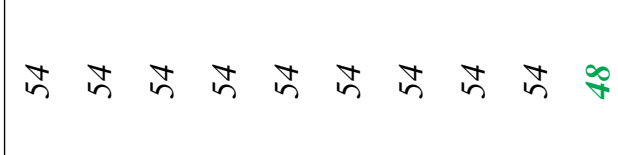 \\
\hline 焉 & $0000000,0 \infty$ \\
\hline 言 & 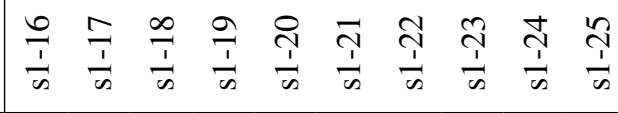 \\
\hline
\end{tabular}




\begin{tabular}{|c|c|c|c|c|c|}
\hline 苛 & & $\begin{array}{ll}\tilde{c} & 0 \\
0 & 0 \\
\tilde{m} & \dddot{0} \\
0 & 0\end{array}$ & 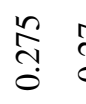 & స̂. & \\
\hline 䔍 & 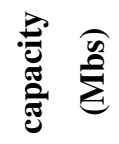 & $\begin{array}{ll}0 & 0 \\
\dot{+} & \stackrel{0}{0} \\
= & 0 \\
=\end{array}$ & 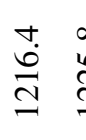 & $\begin{array}{l}\infty \\
\ddot{n} \\
\end{array}$ & $\begin{array}{l}\infty \\
\stackrel{\infty}{\sim} \\
\simeq\end{array}$ \\
\hline 焉 & 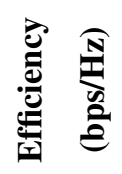 & $\stackrel{\infty}{0}=$ & $\stackrel{9}{=}$ & $\stackrel{ \pm}{=}$ & $\stackrel{n}{=}$ \\
\hline 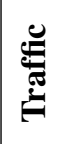 & 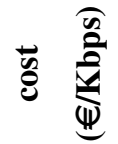 & $\begin{array}{ll}\sqrt{n} & \infty \\
m & n \\
0 & 0\end{array}$ & 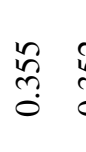 & ח̃ & 年 \\
\hline $\bar{\Xi}$ & 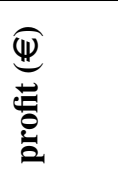 & 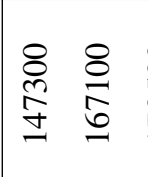 & $\begin{array}{ll}8 & \delta \\
n & \delta \\
& \vdots\end{array}$ & 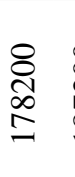 & 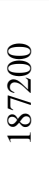 \\
\hline 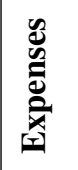 & $\overleftrightarrow{\Psi}$ & 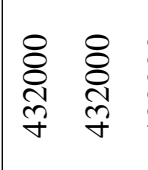 & 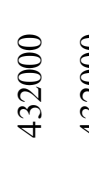 & 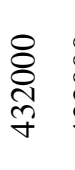 & 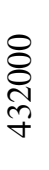 \\
\hline : & $\widehat{\theta}$ & 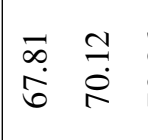 & $\begin{array}{l}\infty \\
\infty \\
\stackrel{2}{\infty}:\end{array}$ & $\underset{\dot{R}}{\vec{r}}$ & 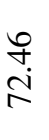 \\
\hline ن & $\widehat{\theta}$ & 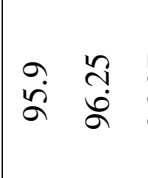 & స઼. & ๙ેे & \begin{tabular}{l}
$\infty$ \\
\multirow{1}{*}{} \\
$\infty$ \\
$\infty$
\end{tabular} \\
\hline 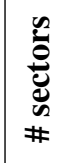 & & in in & in i & in & in \\
\hline $\begin{array}{l}\underbrace{n}_{0} \\
\# \\
\#\end{array}$ & & $a \quad a$ & $a$ & $a$ & $a$ \\
\hline$\Xi$ & & $\begin{array}{ll}0 & 0 \\
\frac{1}{n} & \frac{1}{n}\end{array}$ & $\frac{1}{n}$ & $\begin{array}{l}0 \\
\frac{1}{n}\end{array}$ & $\frac{0}{\frac{1}{n}}$ \\
\hline$\frac{1}{a}$ & & 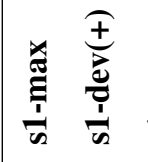 & 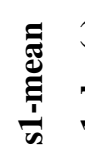 & $\underset{\frac{1}{d}}{\frac{i}{a}}$ & $\frac{\Xi}{\vec{B}}$ \\
\hline
\end{tabular}




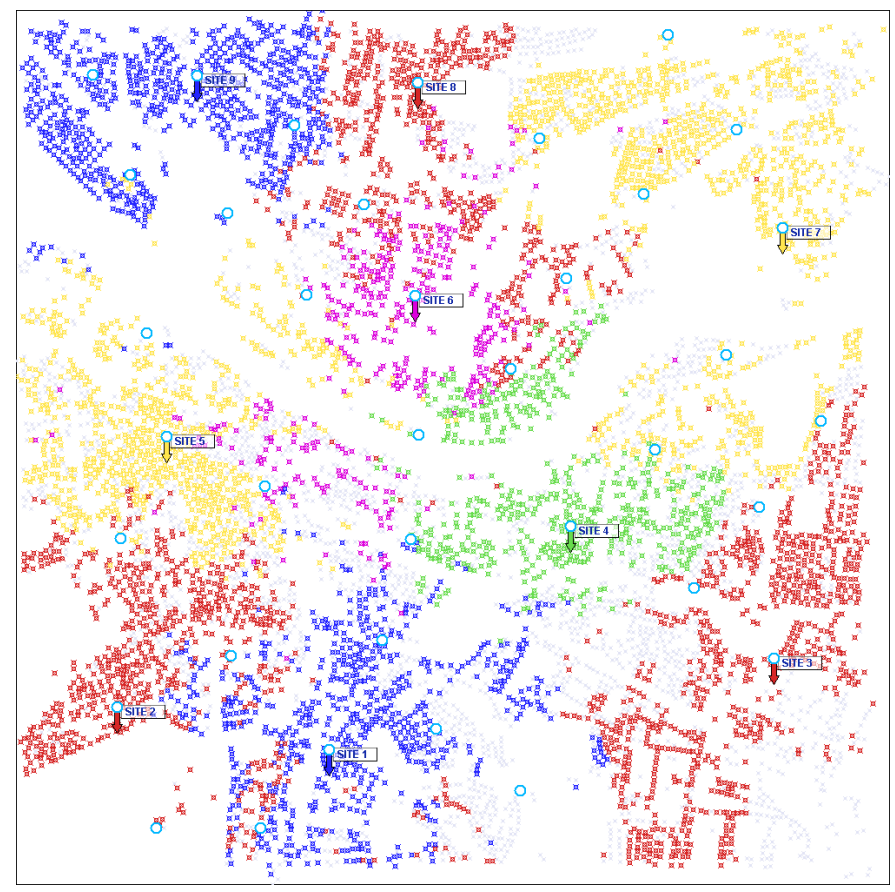

Figure 7.2: Stage 1 network plan - s1-max

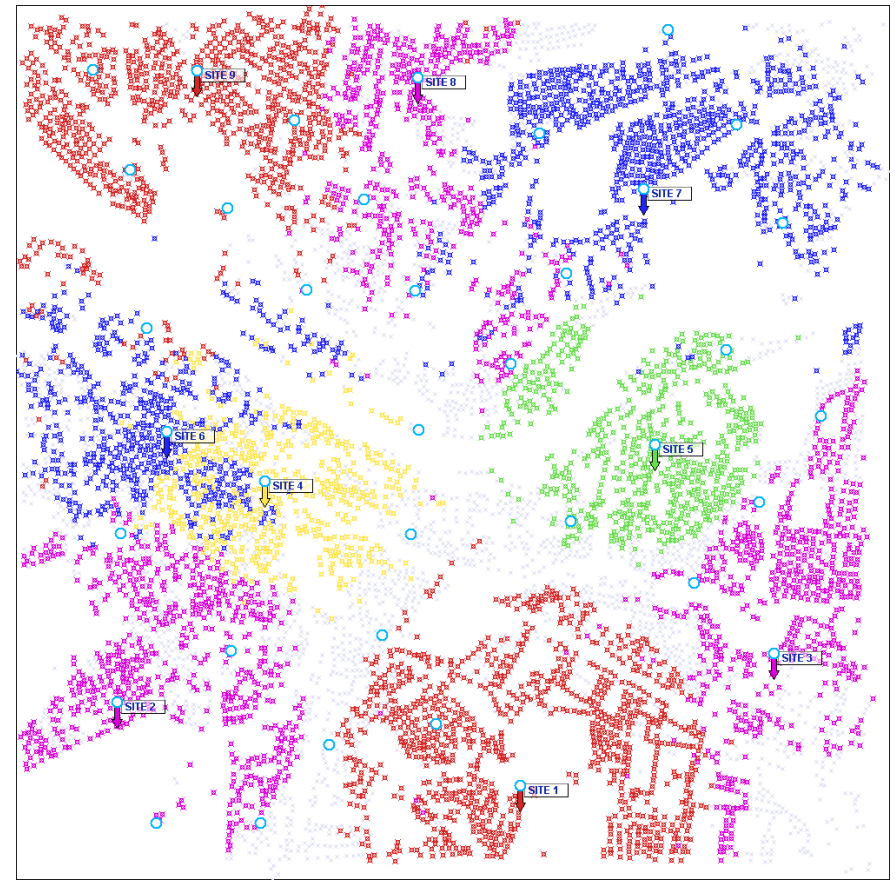

Figure 7.3: Stage 1 network plan - s1-dev(+) 


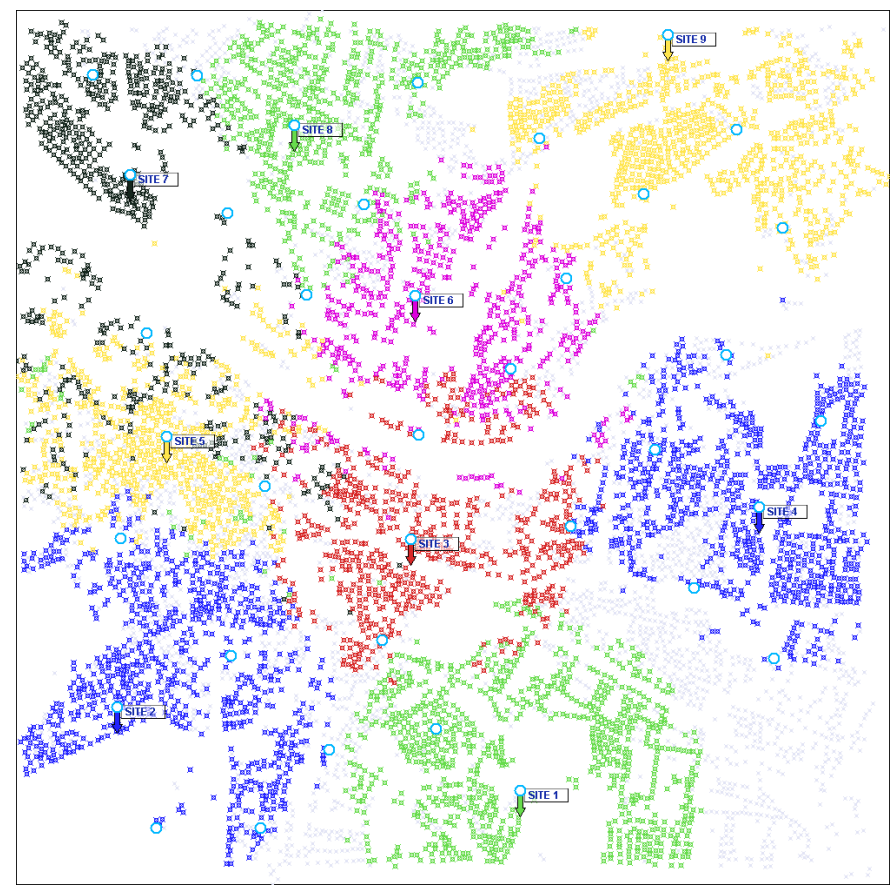

Figure 7.4: Stage 1 network plan - s1-mean

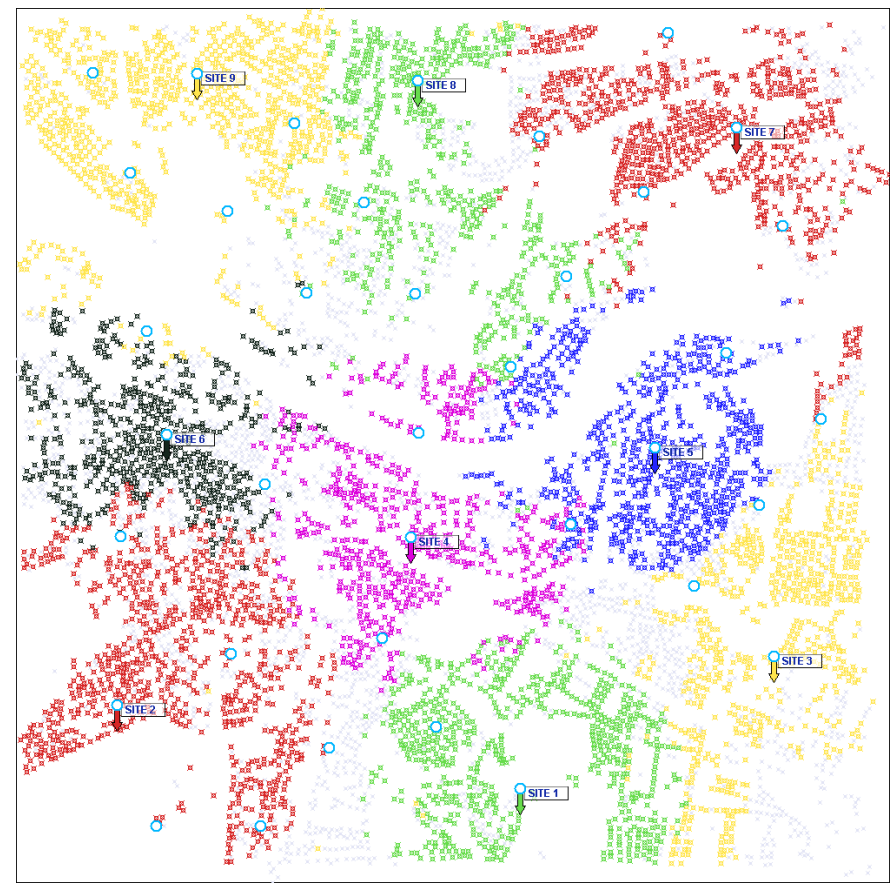

Figure 7.5: Stage 1 network plan - s1-dev(-) 


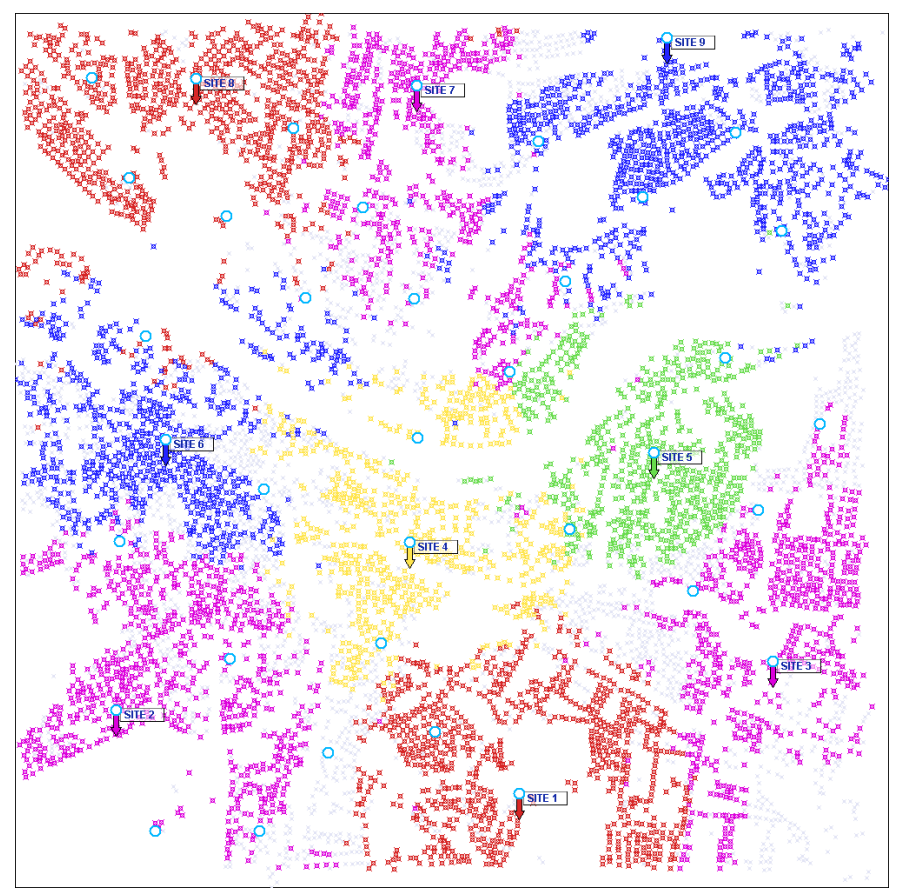

Figure 7.6: Stage 1 network plan - s1-min 
The purpose of Stage 2 as outlined in Section 6.1 is to produce similarly structured cell plans as in Stage 1 while maximizing the overall network service level. For each of the five scenarios chosen from Stage 1 five runs were performed for sectorization. The resulting network designs are presented in Table 7.8 where $\max$ and min indicate the worst and the best network instances respectively from the 5 generated plans. Table 7.9 shows the overall statistical analysis of the network results. It can be seen that the overall trend in terms of the objective function score has been preserved, where the s2-1 network has the worst mean and s2-5 the best. The most important factor in these statistics however is the service gain of Stage 2. The reason that service level was selected as the main indicator is that the expenditure of the networks do not change in Stage 2, indicating that the remaining KPIs will change according to the variation of service level. This indicator shows how the service level of the network plans has changed in comparison to Stage 1 results. In order to calculate this change the mean service level of the 5 networks generated in Stage 2 were compared to the service level of the corresponding selected network plan from Stage 1. A negative value indicates that, on average, the service level of the Stage 2 networks has reduced in comparison to Stage 1, and increased for a positive value. The most important factor however is not the increase or decrease but the amount of change of this indicator after performing sectorization (with maximum difference shown in the s2-5 scenario of $-1.47 \%$, and on average of just $0.656 \%$ ). This indicates that despite the fact that Stage 1, which neither performs the PermBase assignment to the base station sectors, nor the slot allocation to users (in comparison to Stages 2 and 3), and therefore performs without realistic SINR calculation, produces network plans which are well correlated with the results of Stage 2.

Furthermore, five network designs were selected for the subsequent and final modifications from each scenario with minimum value of the objective function. The graphical representations of the selected network plans can be seen on Figures 7.7-7.11 


\begin{tabular}{|c|c|c|c|c|c|c|}
\hline 苞 & & $\stackrel{\substack{I \\
0}}{0}$ & $\stackrel{\substack{n \\
\hdashline}}{\stackrel{9}{0}}$ & $\frac{n}{i} \frac{F}{0}$ & 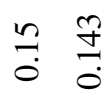 & 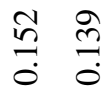 \\
\hline อี & 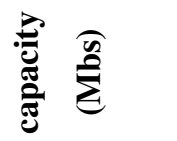 & $\begin{array}{ll}n & \infty \\
\infty & \dot{ \pm} \\
\cong & \Xi \\
= & =\end{array}$ & 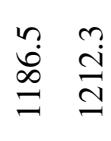 & 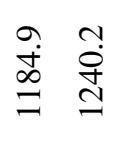 & 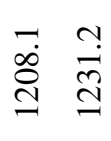 & 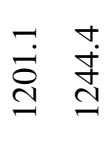 \\
\hline 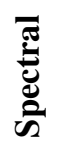 & 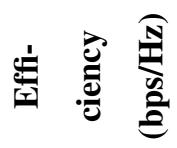 & 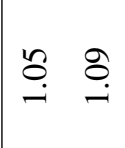 & $\exists \stackrel{\simeq}{=}$ & $=\stackrel{n}{=}$ & $\stackrel{\Xi}{\leftrightarrows}$ & $\exists \stackrel{n}{=}$ \\
\hline 总 & 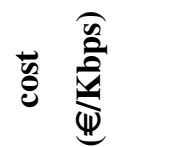 & 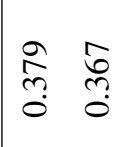 & 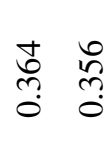 & 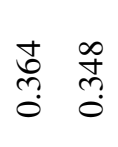 & $\begin{array}{cc}\hat{n} & n \\
n & n \\
0 & 0\end{array}$ & 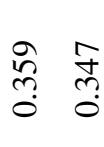 \\
\hline 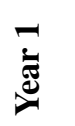 & $\stackrel{\vec{E}}{\vec{E}}$ & 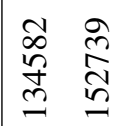 & 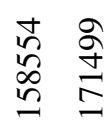 & 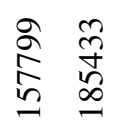 & 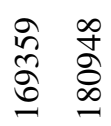 & 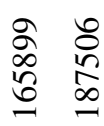 \\
\hline 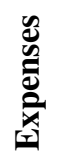 & $\mathbb{\Psi}$ & 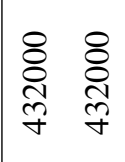 & 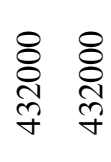 & 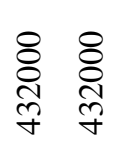 & 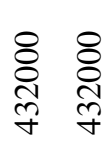 & 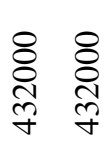 \\
\hline 这 & eq & \begin{tabular}{ll}
$m$ & \multirow{2}{*}{} \\
$\dot{8}$ & $\infty$ \\
0
\end{tabular} & I. & 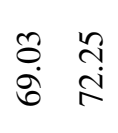 & 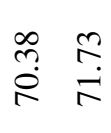 & $\begin{array}{ll}\infty & \stackrel{q}{+} \\
\stackrel{1}{i} & \stackrel{i}{i}\end{array}$ \\
\hline ن & ê & 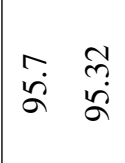 & 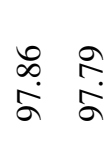 & $\begin{array}{ll}\bar{a} & \hat{a} \\
\text { ڤे }\end{array}$ & 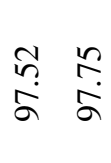 & 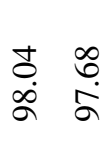 \\
\hline 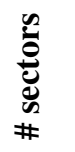 & & in in & $\dot{n}: \vec{n}$ & in & $\dot{n} \vec{n}$ & in in \\
\hline 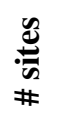 & & $a$ & $a \quad a$ & $a \quad a$ & $a \quad a$ & $a \quad a$ \\
\hline 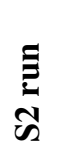 & & 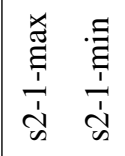 & 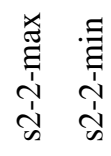 & 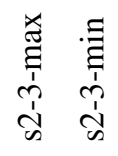 & 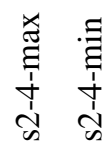 & 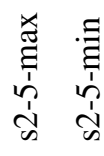 \\
\hline$\frac{n}{\vdots}$ & & 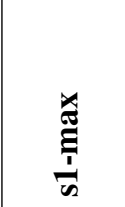 & $\frac{\overparen{f}}{\frac{i}{i}}$ & 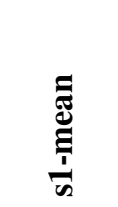 & 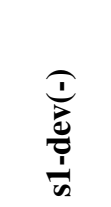 & 章 \\
\hline
\end{tabular}




\begin{tabular}{|c|c|c|c|c|}
\hline S1 networks & S2 networks & S2 stats & S2 score & S2 service gain \\
\hline \multirow{4}{*}{ s1-max } & \multirow{4}{*}{ s2-1 } & $\max$ & 0.1728 & \multirow{3}{*}{$-1.06 \%$} \\
\hline & & mean & 0.1694 & \\
\hline & & $\min$ & 0.1624 & \\
\hline & & \multicolumn{3}{|c|}{ std dev: $0.0042 ; C V: 2.48 \%$} \\
\hline \multirow{4}{*}{ s1-dev(+) } & \multirow{4}{*}{ s2-2 } & $\max$ & 0.1565 & \multirow{3}{*}{$0.11 \%$} \\
\hline & & mean & 0.1515 & \\
\hline & & $\min$ & 0.149 & \\
\hline & & \multicolumn{3}{|c|}{ std dev: $0.0026 ; C V: 1.72 \%$} \\
\hline \multirow{4}{*}{ s1-mean } & \multirow{4}{*}{ s2-3 } & $\max$ & 0.1579 & \multirow{3}{*}{$-0.31 \%$} \\
\hline & & mean & 0.15 & \\
\hline & & $\min$ & 0.1417 & \\
\hline & & \multicolumn{3}{|c|}{ std dev: $0.0057 ; C V: 3.8 \%$} \\
\hline \multirow{4}{*}{ s1-dev(-) } & \multirow{4}{*}{ s2-4 } & $\max$ & 0.1505 & \multirow{3}{*}{$-0.33 \%$} \\
\hline & & mean & 0.1469 & \\
\hline & & $\min$ & 0.1436 & \\
\hline & & \multicolumn{3}{|c|}{ std dev: $0.0029 ; C V: 1.97 \%$} \\
\hline \multirow{4}{*}{ s1-min } & \multirow{4}{*}{ s2-5 } & $\max$ & 0.152 & \multirow{3}{*}{$-1.47 \%$} \\
\hline & & mean & 0.1456 & \\
\hline & & $\min$ & 0.1398 & \\
\hline & & \multicolumn{3}{|c|}{ std dev: $0.0042 ; C V: 2.88 \%$} \\
\hline
\end{tabular}

Table 7.9: Stage 2 statistical analysis of generated networks 


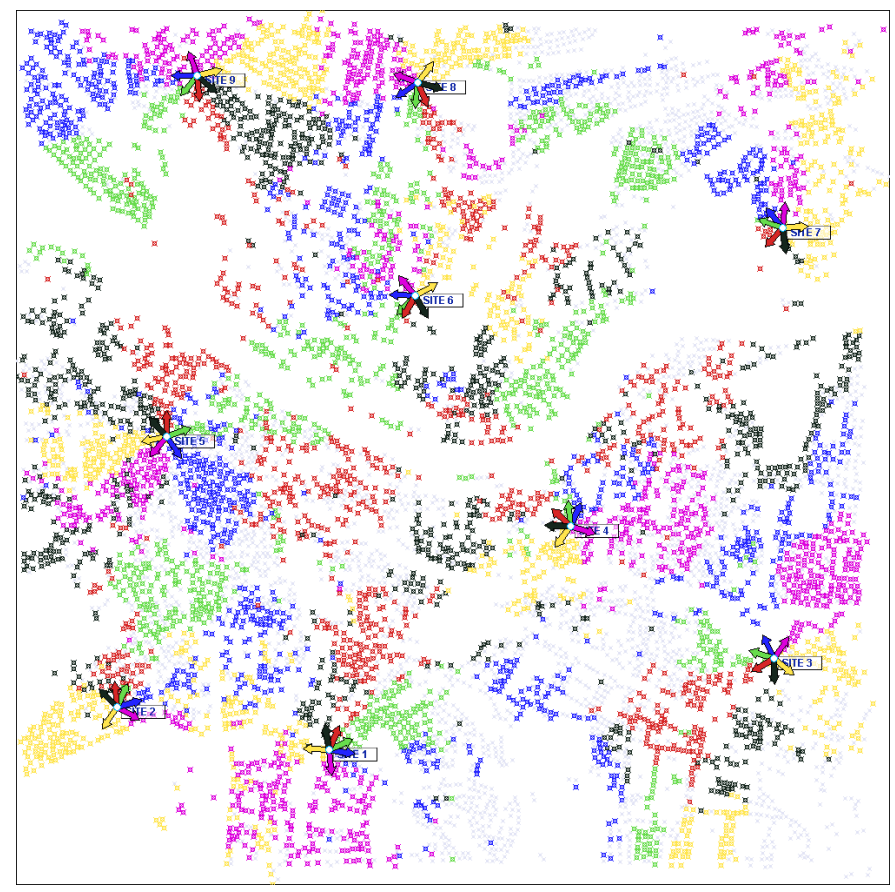

Figure 7.7: Stage 2 network plan - s2-1

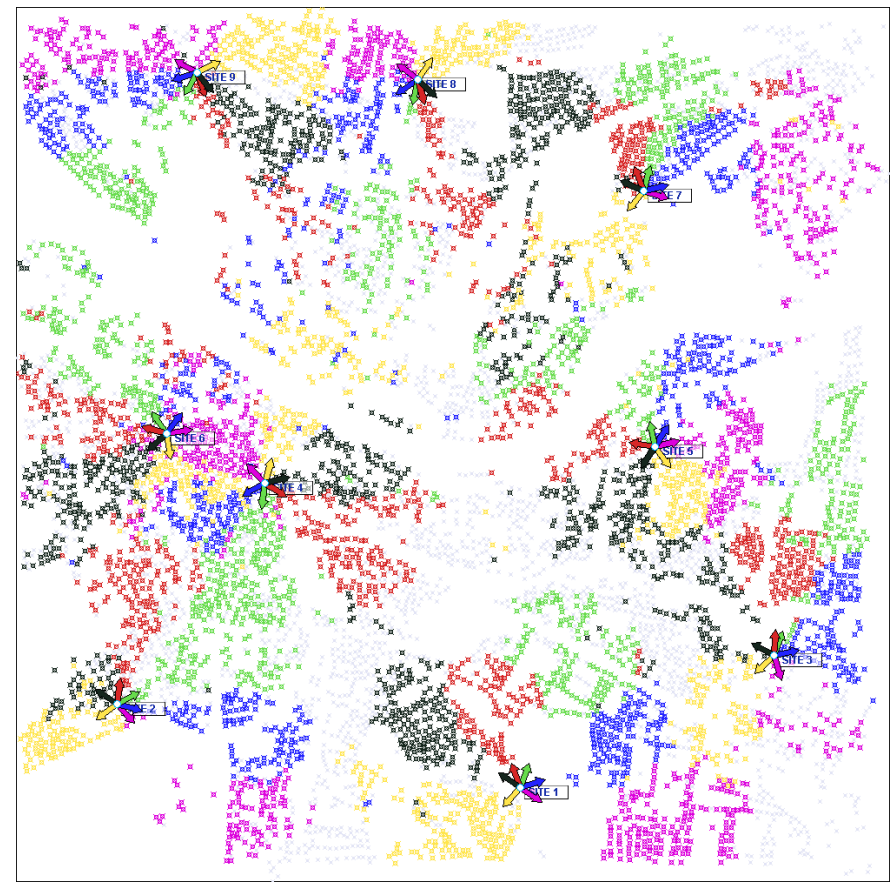

Figure 7.8: Stage 2 network plan - s2-2 


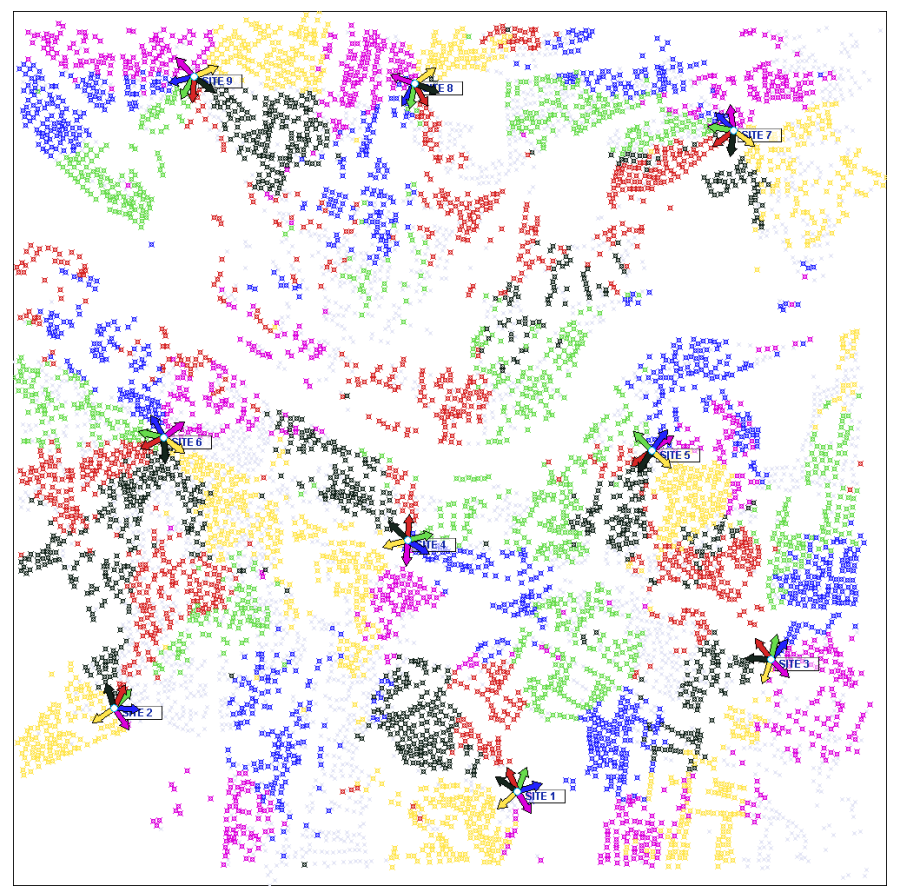

Figure 7.9: Stage 2 network plan - s2-3

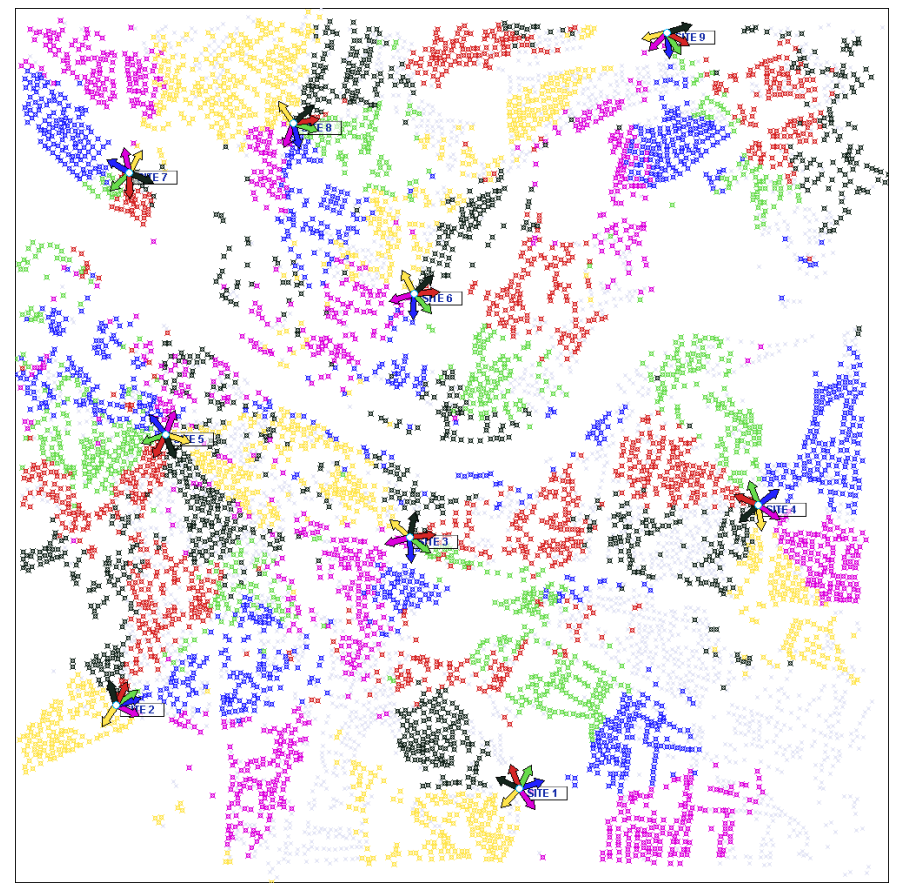

Figure 7.10: Stage 2 network plan - s2-4 


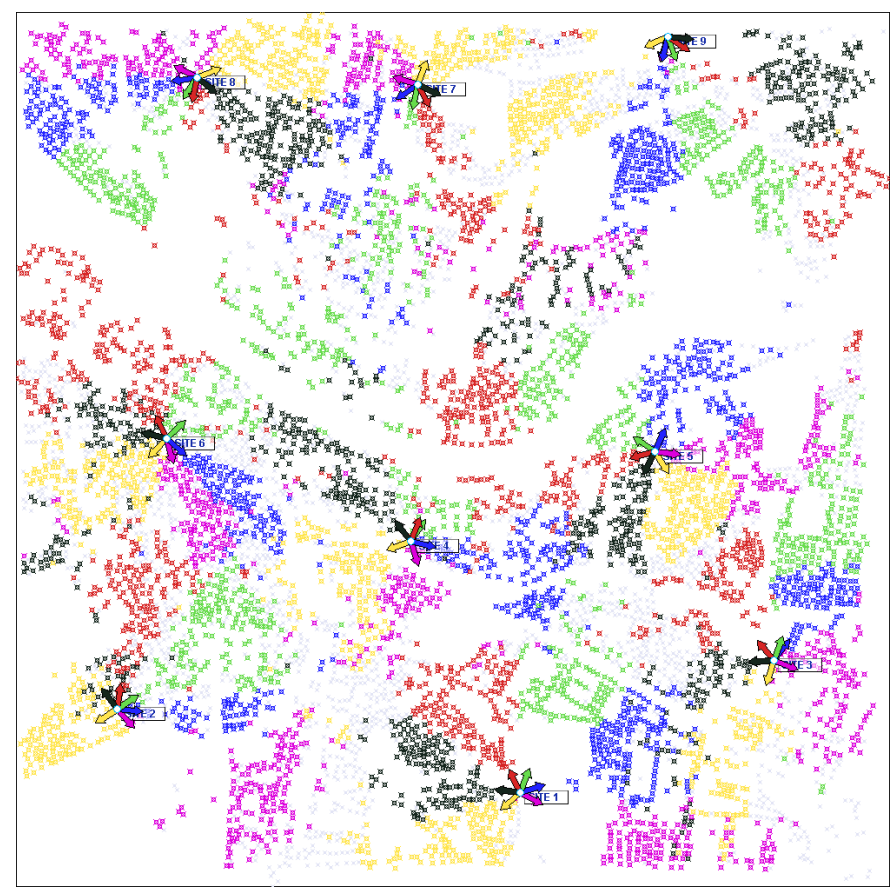

Figure 7.11: Stage 2 network plan - s2-5 
As it was mentioned in Section 6.1 Stage 2 operates using a hill climbing algorithm which converges to a local optimum. The final stage of the optimization framework performs the full range of modifications of the BS sectors in the network using the simulated annealing algorithm in order to achieve the final solution. As in the previous experiments 5 trails were performed for each of the best networks obtained in Stage 2 (s2-1-min, s2-2-min, s2-3-min, s2-4-min, s2-5-min). These generated network plans are presented in Table 7.10. Comparing results of Stage 2 and Stage 3 it can be noted that the improvements have been achieved in all the key performance indicators while retaining the coverage level above $95 \%$. The main statistics as well as the service gain from Stage 3 are presented in Table 7.11. As the average service gain is 3.3\% this may be interpreted as an insignificant improvement. However, this trend once again outlines the value of fine tuning the parameters in Stage 1, and the good sectorization procedures in Stage 2, which in turn leave little room for improvement in the final network optimization stage. This conclusion is tested in the following sections with the final comparison of all experimental results presented in Section 7.7.

Another factor to evaluate the performance of the optimizations stages is a computational time and although this indicator is debatable as it depends on the programming skills of a designer of the system, it can show the relative timings of the stages. Stage 1 on average generates network plans in the time range of 15-20 minutes, whereas it takes 1 hour 30 minutes and 2 hours to execute Stages 2 and 3 respectively. From these figures one conclusion can be made that Stage 1 is a perfect contender for the use as a WiMAX dimensioning tool as it produces good quality results in a relatively short period of time.

Lastly, the graphical representations of the final network plans can be seen on Figures 7.12-7.16. 


\begin{tabular}{|c|c|c|c|c|c|c|}
\hline : & & $\begin{array}{ll}\vec{m} & \infty \\
& \stackrel{m}{0}\end{array}$ & \begin{tabular}{ll}
$\stackrel{\hat{~}}{\hat{n}}$ & \multirow{0}{0}{} \\
0
\end{tabular} & 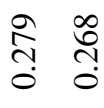 & 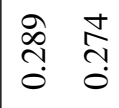 & 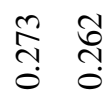 \\
\hline 产 & 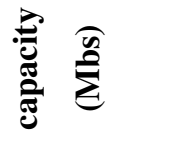 & $\stackrel{\stackrel{\mathscr{D}}{\infty}}{\stackrel{\Xi}{\beth}}$ & 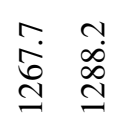 & 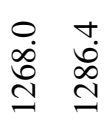 & 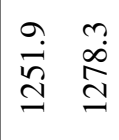 & 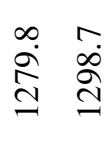 \\
\hline 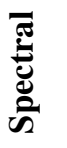 & 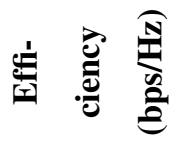 & $=\stackrel{9}{=}$ & $\cong$ & 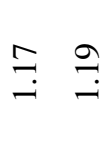 & $\stackrel{0}{\stackrel{0}{=}} \stackrel{\infty}{=}$ & $\stackrel{\cong}{\leftrightarrows}$ \\
\hline 恼 & 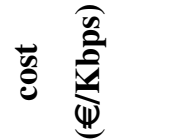 & 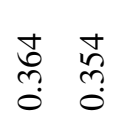 & $\begin{array}{cc}\tilde{n} & \text { ñ } \\
0 & 0\end{array}$ & 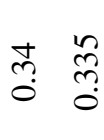 & 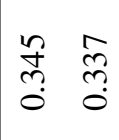 & $\stackrel{n}{m} \tilde{m}$ \\
\hline $\bar{\varpi}$ & 竞 & 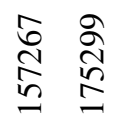 & 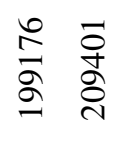 & 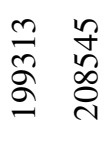 & 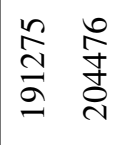 & 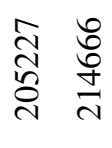 \\
\hline 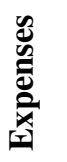 & $\overparen{\Psi}$ & 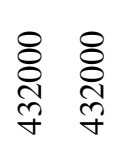 & 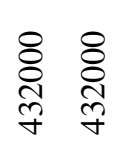 & 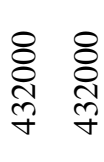 & 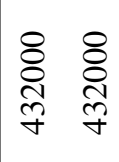 & 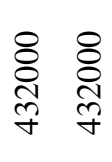 \\
\hline 赵 & to & $\begin{array}{ll}\hat{\alpha} & \hat{0} \\
\hat{\infty} & \vec{\nabla}\end{array}$ & $\begin{array}{ll}n & \vdots \\
\infty & i \\
& \stackrel{n}{r}\end{array}$ & 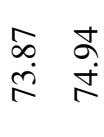 & 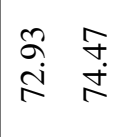 & $\begin{array}{ll}0 \\
\stackrel{2}{+} \\
\stackrel{i}{r}\end{array}$ \\
\hline 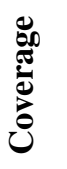 & $\widehat{e}$ & $\begin{array}{ll}\stackrel{n}{n} \\
\stackrel{n}{2}\end{array}$ & $\begin{array}{cc}\stackrel{1}{0} \\
\stackrel{\infty}{\infty}\end{array}$ & $\begin{array}{ll}\text { ते } & + \\
\infty & \infty \\
\curvearrowright\end{array}$ & $\begin{array}{cc}\tilde{a} & \bar{a} \\
\hat{a} & \stackrel{a}{a}\end{array}$ & 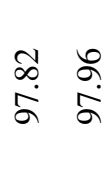 \\
\hline 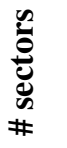 & & in & in in & in in & 苫 in & in \\
\hline 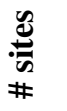 & & $a \quad a$ & $a \quad a$ & $a \quad a$ & $a r$ & $a \quad a$ \\
\hline 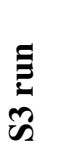 & & 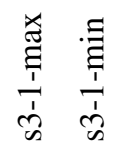 & 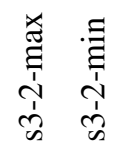 & 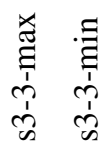 & 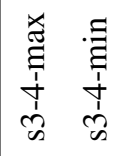 & 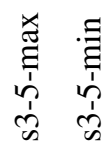 \\
\hline 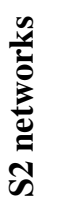 & & 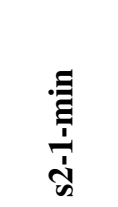 & 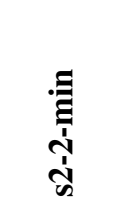 & 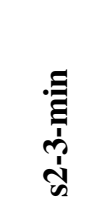 & 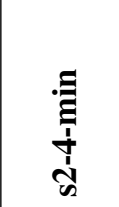 & 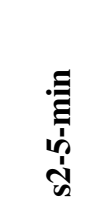 \\
\hline
\end{tabular}




\begin{tabular}{|c|c|c|c|c|}
\hline S2 networks & S3 networks & S3 stats & S3 score & S3 service gain \\
\hline \multirow{4}{*}{ s2-1 } & \multirow{4}{*}{ s3-1 } & $\max$ & 0.3315 & \multirow{3}{*}{$1.91 \%$} \\
\hline & & mean & 0.3226 & \\
\hline & & $\min$ & 0.3086 & \\
\hline & & \multicolumn{3}{|c|}{ std dev: $0.00841 ; C V: 2.61 \%$} \\
\hline \multirow{4}{*}{ s2-2 } & \multirow{4}{*}{ s3-2 } & $\max$ & 0.2796 & \multirow{3}{*}{$5.25 \%$} \\
\hline & & mean & 0.2737 & \\
\hline & & $\min$ & 0.2674 & \\
\hline & & \multicolumn{3}{|c|}{ std dev: $0.00441 ; C V: 1.61 \%$} \\
\hline \multirow{4}{*}{ s2-3 } & \multirow{4}{*}{ s3-3 } & $\max$ & 0.2794 & \multirow{3}{*}{$2.99 \%$} \\
\hline & & mean & 0.2733 & \\
\hline & & $\min$ & 0.2685 & \\
\hline & & \multicolumn{3}{|c|}{ std dev: $0.00432 ; C V: 1.58 \%$} \\
\hline \multirow{4}{*}{ s2-4 } & \multirow{4}{*}{ s3-4 } & $\max$ & 0.2893 & \multirow{3}{*}{$2.98 \%$} \\
\hline & & mean & 0.2797 & \\
\hline & & & & \\
\hline & & \multicolumn{3}{|c|}{ std dev: $0.00541 ; C V: 1.93 \%$} \\
\hline \multirow{4}{*}{ s2-5 } & \multirow{4}{*}{ s3-5 } & $\max$ & 0.2733 & \multirow{3}{*}{$3.39 \%$} \\
\hline & & mean & 0.2686 & \\
\hline & & $\min$ & 0.2621 & \\
\hline & & \multicolumn{3}{|c|}{ std dev: $0.00383 ; C V: 1.43 \%$} \\
\hline
\end{tabular}

Table 7.11: Stage 3 statistical analysis of generated networks 


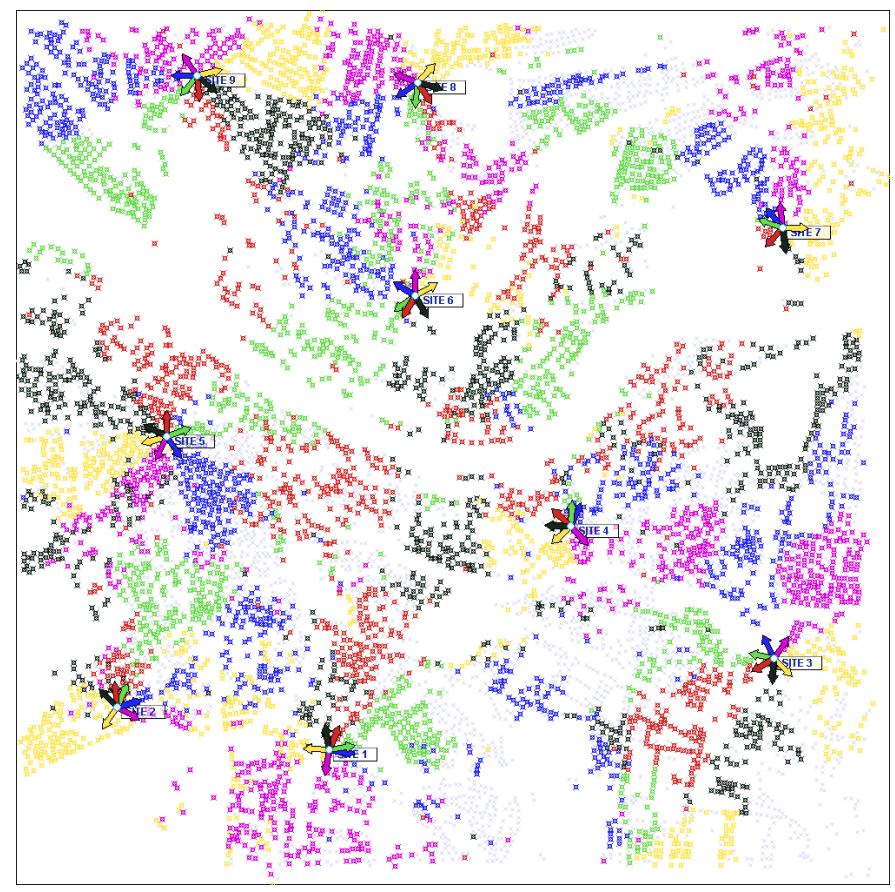

Figure 7.12: Stage 3 network plan - s3-1-min

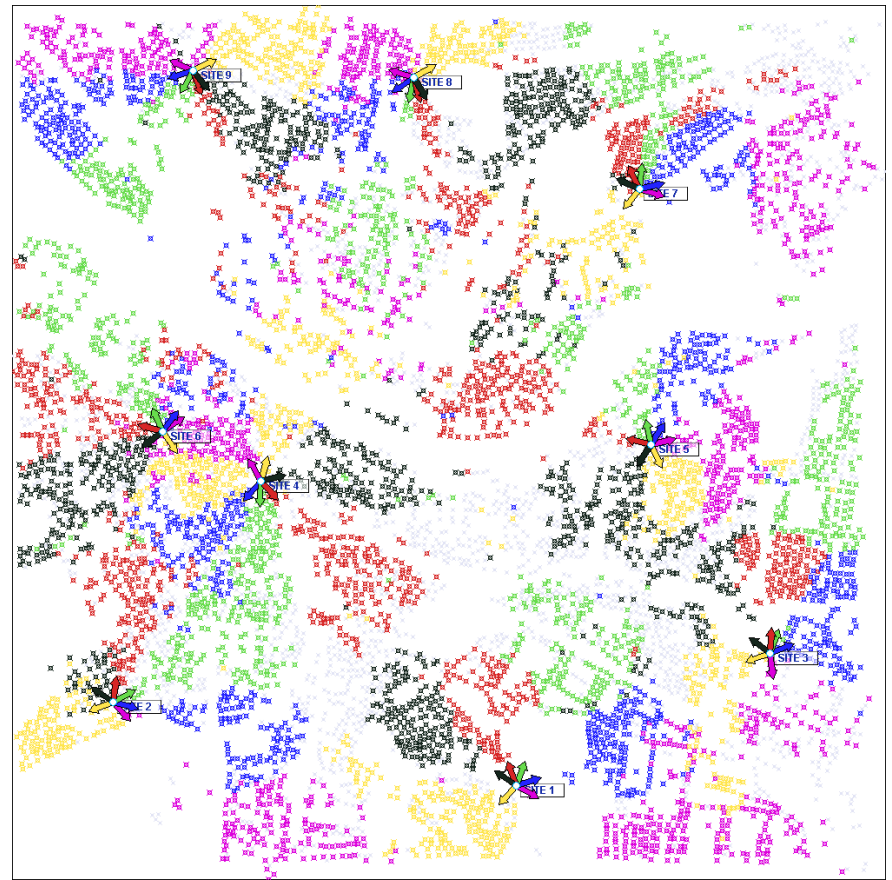

Figure 7.13: Stage 3 network plan - s3-2-min 


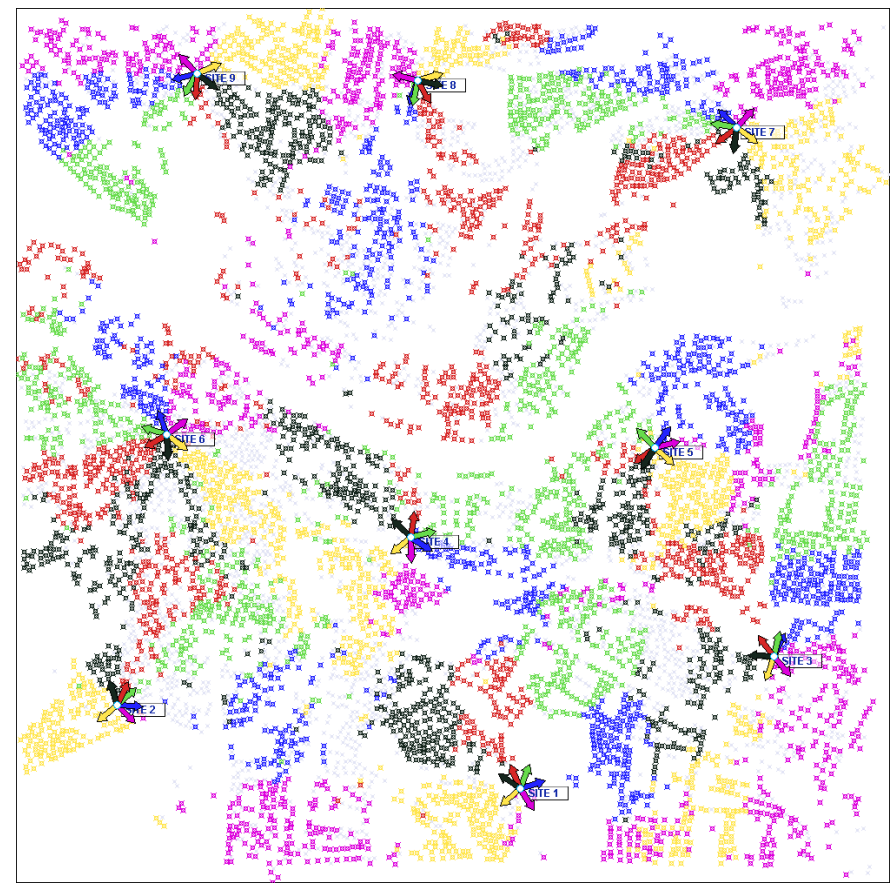

Figure 7.14: Stage 3 network plan - s3-3-min

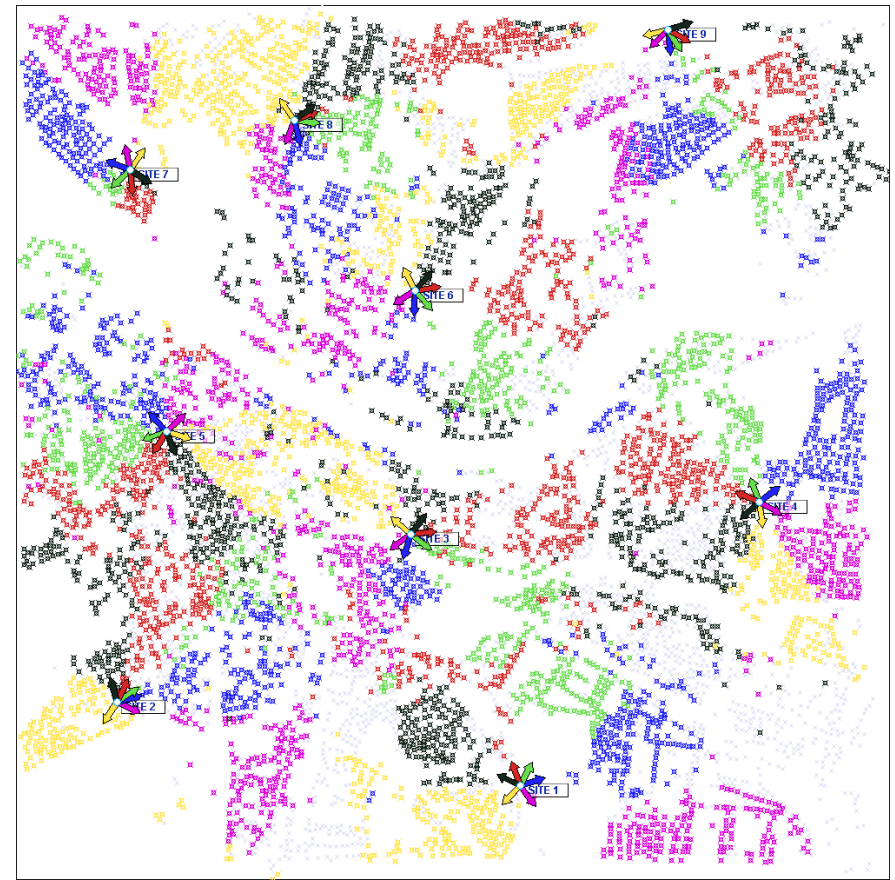

Figure 7.15: Stage 3 network plan - s3-4-min 


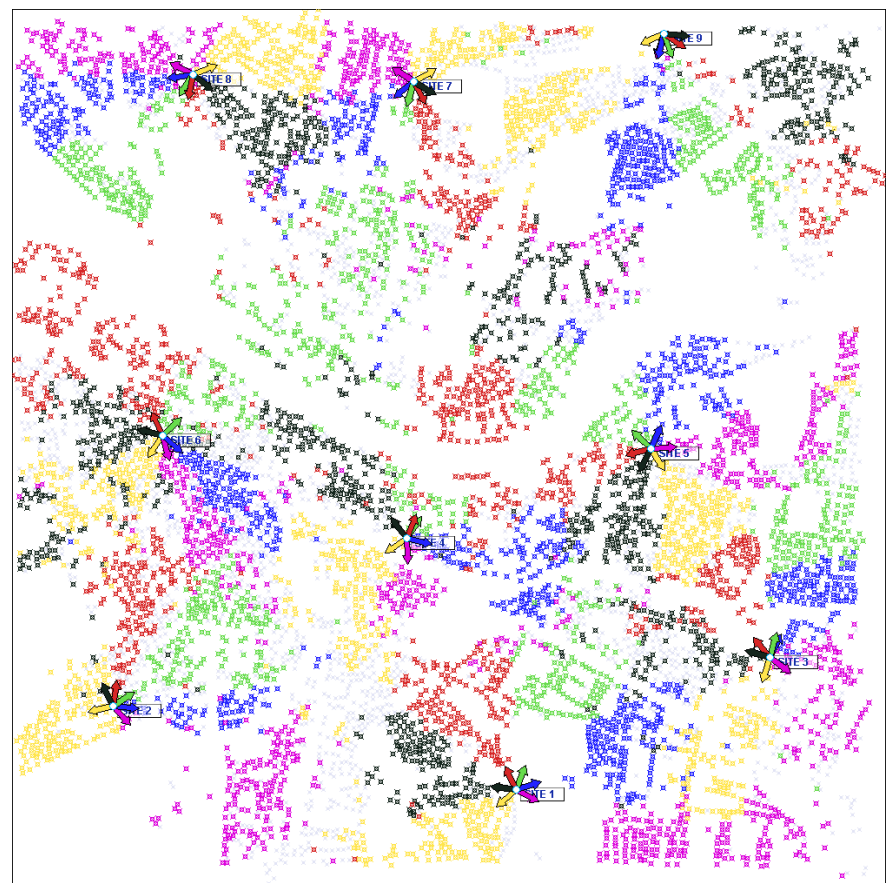

Figure 7.16: Stage 3 network plan - s3-5-min 


\subsection{Network planning without consideration of Perm- Base assignment}

The PermBase is an integer used in the permutation formula (Eq. 7.2) that controls the allocation of subcarriers to a subchannel in the sector of a cell. It implies that the same subchannel in two different sectors, using the same frequency band but different PermBase, will comprise of different subcarriers, thereby reducing interference. In our framework, Stage 1 of the network optimization process does not consider PermBase allocation. Stages 2 and 3 however do perform assignment of different PermBase's to the BSs in order to avoid excessive interference, therefore allowing sectors to communicate with the users on the higher order modulation schemes, thus maximizing the overall network service level. Most of the proposed solutions for WiMAX network planning in the literature do not consider PermBase as a decision variable in the optimization framework. Therefore, in order to see the effect on the results when PermBase allocation is disregarded, the network plans obtained from Stage 3, which are listed in Table 7.10, are re-deployed but with the same PermBase in all the sectors of the networks. The resulting network solutions can be observed in Table 7.12, It is notable that all the KPIs are significantly worse. As the spectral efficiency dropped due to the increased interference level in the networks, it inevitably has a negative impact on the overall service levels, thus reducing the profitability of the networks. The statistical comparison of the network results with and without PermBase allocation is presented in Table 7.13. It can be seen that the performance of the networks has dropped considerably in comparison to our proposed approach which includes PermBase allocation. The average degradation in traffic service level of the users accounts for $-19.28 \%$. 


\begin{tabular}{|c|c|c|c|c|c|}
\hline 离 & 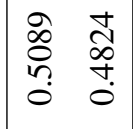 & $\begin{array}{cc}c & a \\
\infty & 0 \\
o & 0 \\
f & 0 \\
0 & 0 \\
0\end{array}$ & 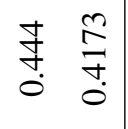 & 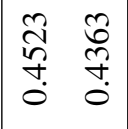 & 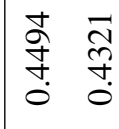 \\
\hline 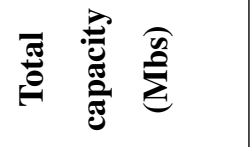 & 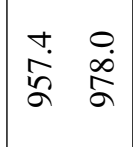 & 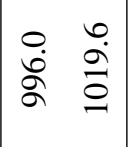 & 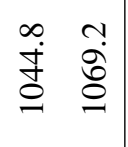 & $\stackrel{\circ}{\circ} \stackrel{\circ}{\vec{\delta}}$ & 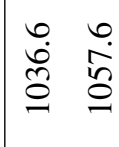 \\
\hline 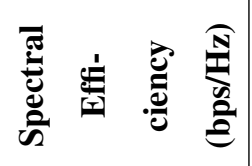 & $\begin{array}{cc}\infty & \bar{a} \\
0 & 0\end{array}$ & $\begin{array}{ll}\delta & t \\
\delta & 0\end{array}$ & $\hat{a} \quad \hat{o}$ & $\begin{array}{ll}\swarrow & 0 \\
0 & 0\end{array}$ & $\left(\begin{array}{ll}0 & \infty \\
\hdashline & 0\end{array}\right.$ \\
\hline 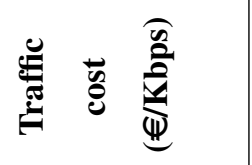 & $\begin{array}{ll}\vec{y} & \overrightarrow{7} \\
0 \\
0 & 0 \\
0\end{array}$ & 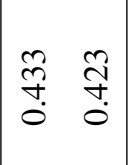 & 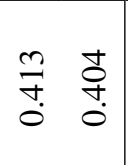 & 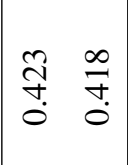 & \begin{tabular}{ll}
0 & $\infty$ \\
\multirow{f}{*}{} & $\stackrel{+}{0}$
\end{tabular} \\
\hline $\overrightarrow{\bar{E}}$ & 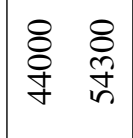 & 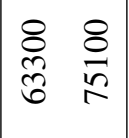 & 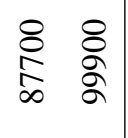 & 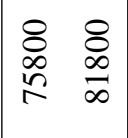 & 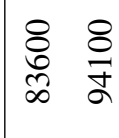 \\
\hline 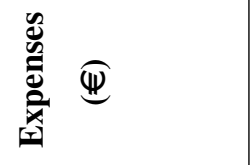 & 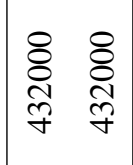 & 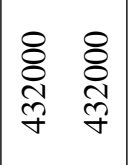 & 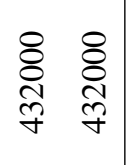 & 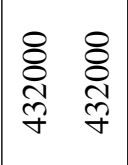 & 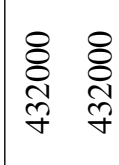 \\
\hline$\widehat{\mathscr{E}}$ & $\begin{array}{ll}\hat{n} & \hat{\sigma} \\
i & \hat{b} \\
i n & i n\end{array}$ & $\begin{array}{ll}\delta & + \\
\infty & \dot{D} \\
i & i n\end{array}$ & 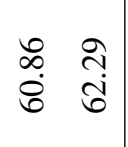 & 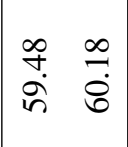 & 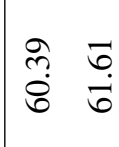 \\
\hline 焉 & $\begin{array}{ll}\hat{\gamma} & 0 \\
\dot{\alpha} & \delta \\
\alpha & \delta\end{array}$ & 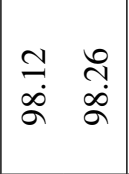 & \begin{tabular}{cc}
+ & \multicolumn{1}{c}{} \\
$\alpha$ & $\dot{\alpha}$ \\
$\alpha$
\end{tabular} & 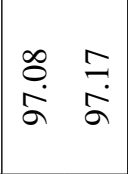 & 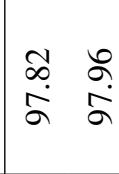 \\
\hline 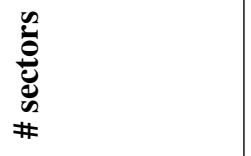 & 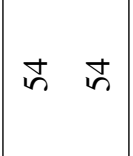 & 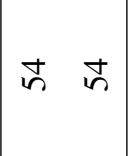 & 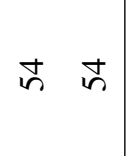 & it is & 点 出 \\
\hline 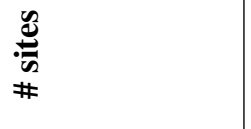 & $a c$ & $\begin{array}{ll}a & a\end{array}$ & $a r$ & $\begin{array}{ll}a & a\end{array}$ & $\begin{array}{ll}a & a\end{array}$ \\
\hline 要 & 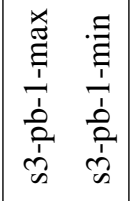 & 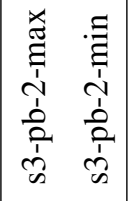 & 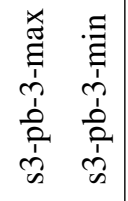 & 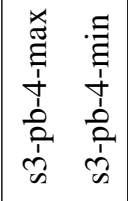 & 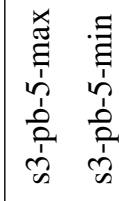 \\
\hline 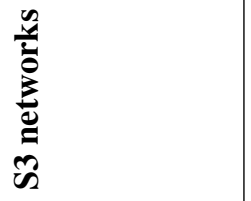 & $\bar{n}$ & ஸे & $\tilde{n}$ & $\vec{D}$ & कृ \\
\hline
\end{tabular}




\begin{tabular}{|c|c|c|c|c|}
\hline S3 networks & S3-PB networks & $\begin{array}{c}\text { S3-PB } \\
\text { stats }\end{array}$ & $\begin{array}{c}\text { S3-PB } \\
\text { score }\end{array}$ & S3-PB service gain \\
\hline \multirow{4}{*}{ s3-1 } & \multirow{4}{*}{ s3-pb-1 } & $\max$ & 0.5089 & \multirow{3}{*}{$-20.25 \%$} \\
\hline & & mean & 0.5002 & \\
\hline & & $\min$ & 0.4824 & \\
\hline & & \multicolumn{3}{|c|}{ std dev: $0.00946 ; C V: 1.89 \%$} \\
\hline \multirow{4}{*}{ s3-2 } & \multirow{4}{*}{ s3-pb-2 } & $\max$ & 0.4587 & \multirow{3}{*}{$-20.86 \%$} \\
\hline & & mean & 0.4489 & \\
\hline & & $\min$ & 0.4369 & \\
\hline & & \multicolumn{3}{|c|}{ std dev: $0.00826 ; C V: 1.84 \%$} \\
\hline \multirow{4}{*}{ s3-3 } & \multirow{4}{*}{ s3-pb-3 } & $\max$ & 0.444 & \multirow{3}{*}{$-17.43 \%$} \\
\hline & & mean & 0.4332 & \\
\hline & & & 0.4173 & \\
\hline & & \multicolumn{3}{|c|}{ std dev: $0.00945 ; C V: 2.18 \%$} \\
\hline \multirow{4}{*}{ s3-4 } & \multirow{4}{*}{ s3-pb-4 } & $\max$ & 0.4523 & \multirow{3}{*}{$-19.3 \%$} \\
\hline & & mean & 0.446 & \\
\hline & & $\min$ & 0.4363 & \\
\hline & & \multicolumn{3}{|c|}{ std dev: $0.00536 ; C V: 1.2 \%$} \\
\hline \multirow{4}{*}{ s3-5 } & \multirow{4}{*}{ s3-pb-5 } & $\max$ & 0.4494 & \multirow{3}{*}{$-18.58 \%$} \\
\hline & & mean & 0.4423 & \\
\hline & & & 0.4321 & \\
\hline & & \multicolumn{3}{|c|}{ std dev: $0.00615 ; C V: 1.39 \%$} \\
\hline
\end{tabular}

Table 7.13: Stage 3 statistical analysis of networks without PermBase assignment 


\subsection{Network planning with simplified Stage 2 operation}

All the stages in the proposed optimization framework for WiMAX network planning are designed to solve a specific set of criteria, and when executed in the defined order they produce efficient and cost-optimized results. Stage 1 aims to identify the optimal site locations for BS deployment, as well as the required amount of equipment, together with the sets of users recommended for service from each site location. Stage 2 performs initial sectorization of omni-directional BSs which serves as a good starting point for the subsequent and final stage. In order to evaluate the effectiveness and benefit of the Stage 2 sectorization process the following experiment is conducted. First, the network plans generated in Stage 1, Table 7.7, instead of being processed by Stage 2 , were sectorized with the default settings and equally separated in the azimuth plane (1 sectorization trial for each network from Stage 1). The results of this simple operation are presented in Table 7.14. This table also provides comparative analysis to the performance of this operation to the results of the Stage 1 and 2 in terms of the service gain. The service level is $17.51 \%$ lower than in Stage 1 and $17.48 \%$ lower than in Stage 2, which indicates the benefit of Stage 2. Secondly, having omitted the full sectorization process these newly generated network plans are passed to Stage 3 in order to maximize each KPI. As in Section 7.2 5 trials were executed for each network plan. The final networks are listed in Table 7.15 and their statistical evaluation in Table 7.16. It can be seen that without Stage 2, which intelligently sectorizes omni base stations and pre-configures all network sectors, Stage 3 on its own does not cope with the complexity of the problem and produces results on average $11.35 \%$ worse than from the proposed framework. This can be explained by the amount of decision variables, specifically the added problem of finding optimal azimuths for sectorized BSs. 


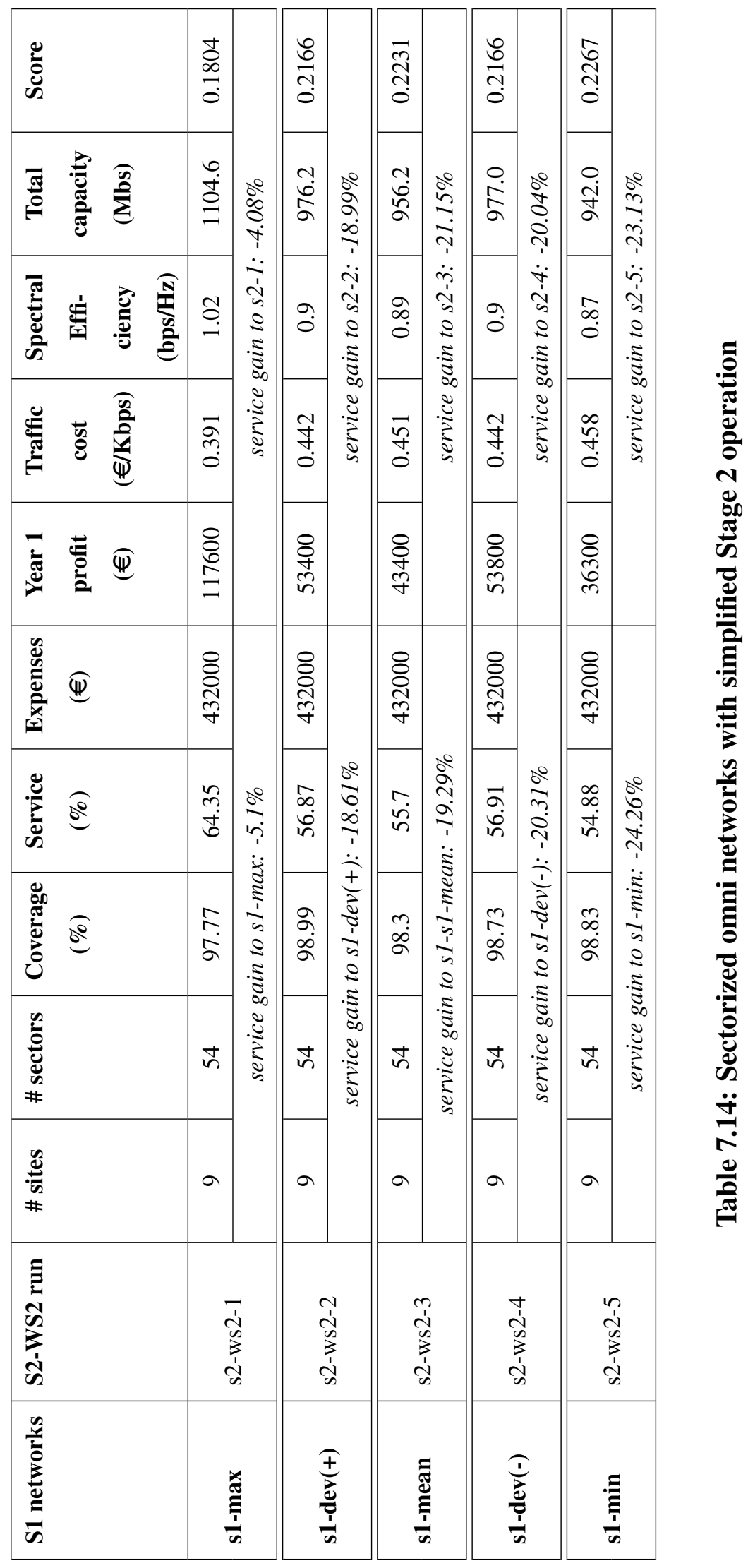




\begin{tabular}{|c|c|c|c|c|c|c|c|}
\hline 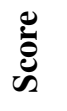 & & 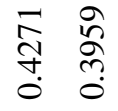 & 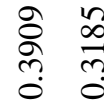 & $\stackrel{n}{\hat{n}}$ & & 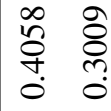 & 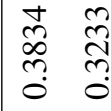 \\
\hline बै & 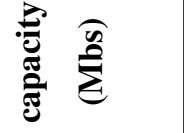 & \begin{tabular}{ll}
+ & \multirow{1}{*}{} \\
$\stackrel{0}{0}$ & $\infty$ \\
0 & 0
\end{tabular} & $\begin{array}{l}\stackrel{0}{+} \\
\stackrel{5}{0} \\
\stackrel{0}{=}\end{array}$ & $\stackrel{0}{0}$ & 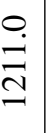 & $\begin{array}{ll}\infty & 0 \\
\dot{v} & \dot{m} \\
\stackrel{\Xi}{0} & \underline{\Xi}\end{array}$ & $\begin{array}{ll}\infty & 0 \\
\dot{0} & \dot{0} \\
0 & \infty \\
0 & \stackrel{=}{=}\end{array}$ \\
\hline 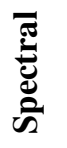 & 竞 & 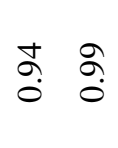 & $\stackrel{\circ}{\circ}=$ & $\stackrel{\circ}{\circ}$ & $\stackrel{\simeq}{=}$ & $\hat{a} \stackrel{ \pm}{\vdots}$ & $\underset{0}{-}$ \\
\hline 总 & 鸟 & 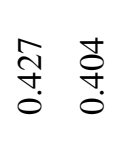 & 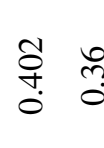 & \begin{tabular}{ll}
0 \\
\multirow{0}{*}{} \\
$\stackrel{0}{0}$
\end{tabular} & 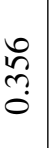 & $\begin{array}{ll}m & n \\
\stackrel{\sigma}{*} & \tilde{0}\end{array}$ & 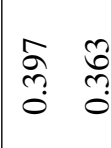 \\
\hline$\stackrel{\bar{E}}{\grave{E}}$ & $\underset{0}{\mathbb{0}}$ & 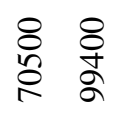 & 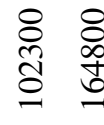 & $\begin{array}{ll}8 & \\
\infty & 0 \\
\sigma & 0\end{array}$ & 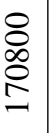 & $\begin{array}{ll}8 & 8 \\
\infty & = \\
\infty & \infty \\
\infty & \infty\end{array}$ & 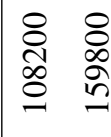 \\
\hline 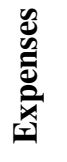 & $\overparen{\Psi}$ & 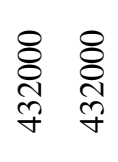 & 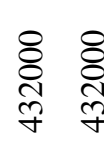 & 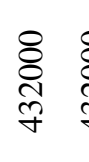 & 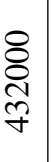 & 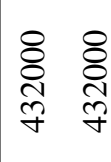 & 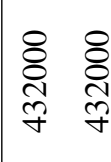 \\
\hline : & $\widehat{\theta}$ & 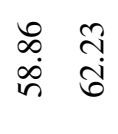 & $\begin{array}{ll}\tilde{n} & 0 \\
\text { กิ } & 8 \\
0\end{array}$ & 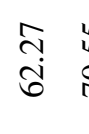 & $\begin{array}{l}n \\
\stackrel{n}{n} \\
2\end{array}$ & 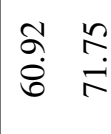 & 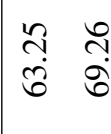 \\
\hline 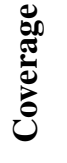 & $\widehat{\theta}$ & 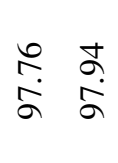 & $\begin{array}{ll}\infty & 0 \\
\circ & 8\end{array}$ & $\begin{array}{ll}n \\
\infty \\
\infty \\
\infty\end{array}$ & $\begin{array}{l}8 \\
0 \\
\infty \\
0\end{array}$ & $\begin{array}{ll}2 & \mathbb{1} \\
\infty & \infty \\
\infty & \infty \\
\infty & \infty\end{array}$ & $\begin{array}{ll}\infty & \infty \\
\infty & \infty \\
\infty & \infty \\
\sigma & \infty\end{array}$ \\
\hline$\#$ & 竧 & in in & in in & in & in & in in & in in \\
\hline $\begin{array}{l}\stackrel{\mathscr{n}}{\mathscr{E}_{n}^{2}} \\
\#\end{array}$ & & $a \quad a$ & $a r$ & $a$ & $a$ & $a r$ & $a r$ \\
\hline 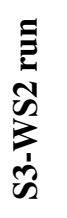 & & 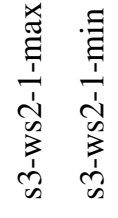 & 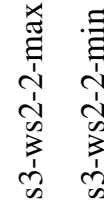 & 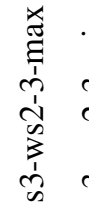 & 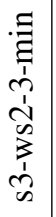 & 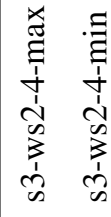 & 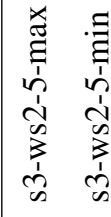 \\
\hline 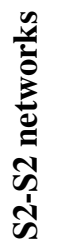 & & 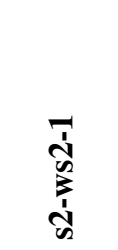 & 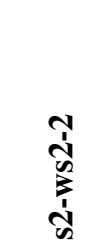 & 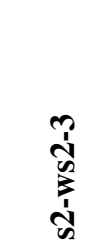 & & 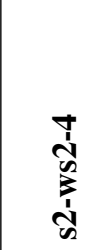 & 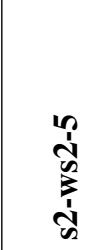 \\
\hline
\end{tabular}




\begin{tabular}{|c|c|c|c|c|}
\hline S3 networks & S3-WS2 networks & $\begin{array}{c}\text { S3-WS2 } \\
\text { stats }\end{array}$ & $\begin{array}{c}\text { S3-WS2 } \\
\text { score }\end{array}$ & S3-WS2 service gain \\
\hline \multirow{4}{*}{ s3-1 } & \multirow{4}{*}{ s3-ws2-1 } & $\max$ & 0.4271 & \multirow{3}{*}{$-13.77 \%$} \\
\hline & & mean & 0.4149 & \\
\hline & & $\min$ & 0.3959 & \\
\hline & & \multicolumn{3}{|c|}{ std dev: $0.01064 ; C V: 2.56 \%$} \\
\hline \multirow{4}{*}{ s3-2 } & \multirow{4}{*}{ s3-ws2-2 } & $\max$ & 0.3909 & \multirow{3}{*}{$-12.2 \%$} \\
\hline & & mean & 0.3633 & \\
\hline & & $\min$ & 0.3185 & \\
\hline & & \multicolumn{3}{|c|}{ std dev: $0.02589 ; C V: 7.13 \%$} \\
\hline \multirow{4}{*}{ s3-3 } & \multirow{4}{*}{ s3-ws2-3 } & $\max$ & 0.39352 & \multirow{3}{*}{$-11.16 \%$} \\
\hline & & mean & 0.3548 & \\
\hline & & $\min$ & 0.3119 & \\
\hline & & \multicolumn{3}{|c|}{ std dev: $0.03322 ; C V: 9.36 \%$} \\
\hline \multirow{4}{*}{ s3-4 } & \multirow{4}{*}{ s3-ws2-4 } & $\max$ & 0.40584 & \multirow{3}{*}{$-9.06 \%$} \\
\hline & & mean & 0.3443 & \\
\hline & & & & \\
\hline & & \multicolumn{3}{|c|}{ std dev: $0.03407 ; C V: 9.9 \%$} \\
\hline \multirow{4}{*}{ s3-5 } & \multirow{4}{*}{ s3-ws2-5 } & $\max$ & 0.3834 & \multirow{3}{*}{$-10.56 \%$} \\
\hline & & mean & 0.3468 & \\
\hline & & $\min$ & 0.3233 & \\
\hline & & \multicolumn{3}{|c|}{ std dev: $0.02408 ; C V: 6.94 \%$} \\
\hline
\end{tabular}

Table 7.16: Stage 3 statistical analysis of networks after simplified Stage 2 operation. 


\subsection{Network planning with different interference set- tings in Stage 1}

As described in Section 5.1 the formulation of interference in the initial stage of network planning when the omni-directional base stations are used for the simulation of the sectorized cells, raises the issue of accuracy in the SINR calculations and therefore the determination of suitable modulation and coding schemes in order to achieve the highest DL bit-rate possible. This issue was due to the fact that no slot assignment is considered in Stage 1. The proposed solution to this was connection the capacity of the interfering omni base stations, and the distance to users from these cells, to estimate the amount of interference these BSs created. In Chapter 5 the solution to this issue resulted in the formation of Table 5.1 and the modification of the SINR equations specific to Stage 1, i.e. eq. 5.1 and 5.2. The results presented in Section 7.2 have shown the high level of correlation between network plans produced in Stages 1 and 2, where the difference between the overall network service levels in both stages accounted for less than $1 \%$. However, in order to show that the model of interference presented in Stage 1 is correct we conducted experiments which reveal the impact of changing the parameters in Table 5.1, later referred to as the capacity-interference parameter. This look up table provides the number of sectors which would cause interference depending on the capacity of the base station and distance between the user and interfering cell, which is used in the calculation of the SINR. Two cell plans were produced in Stage 1 fixing this parameter, regardless of BS capacity or the distance to a user, at a level of 0.5 , representing the lowest amount of interference and at a value of 6 for the highest interference. This indicates that when a user is considered for service by a BS, the number of interfering sectors from each interfering BS will be fixed to 0.5 and 6 respectively. The results of this experiment are listed in Table 7.17. It can be seen that the two networks are very different, s1-i05 which used the capacity-interference parameter of 0.5 has produced high service level with relatively low expense activating only 8 sites and 48 sectors in total, as well as using the spectrum more efficiently. The second net- 
work plan, s1-i6, which used the parameter of 6, activated 9 sites and 54 sectors, but with much lower service level due to the high interference created in the network by amending the capacity-interference parameter. This results in a low spectral efficiency and poor profitability of the network. However, it is more interesting to see the impact on the Stage 2 results which was created by this amendment. The sectorized network plans are shown in Tables 7.18 and 7.19 . Once again 5 trials were performed in Stage 2 for each network plan generated by Stage 1, with the best and the worst networks listed in the tables. It is clear that lowering the capacity-interference parameter in s1-i05 case to a minimum level resulted in unrealistic evaluation of interference in Stage 1 which led to overestimation of spectral efficiency of the base stations and their capacities. This however was corrected in the s2-i05 cell plans which employ a realistic SINR calculation model resulting in the loss of service level on average by $-12.14 \%$. With the capacity-interference parameter set to 6 however, the results are quite the opposite. The higher interference introduced in Stage 1 resulted in generating the network plans with underestimation of the KPIs which also reflected in the poor selection of site locations. This can be seen on the results of the sectorized cell plans after performing Stage 2. In comparison to the 0.5 case, the increase of capacity-interference parameter results in the improvement of the service level after sectorization.

Both of these experiments show a considerable difference in the results between Stages 1 and 2 which makes the performance of the initial planning stage unreliable in this case and proving that with the use of settings provided in Table 5.1 Stage 1 generates near optimal WiMAX network plans perfectly suitable for network dimensioning purposes, as well as serving as a good starting point for the subsequent modification in Stages 2 and 3. The graphical representation of the generated cell plans can be seen on Figures 7.17 and 7.18 for Stage 1, and Figures 7.19 and 7.20 for Stage 2. 


\begin{tabular}{|c|c|c|}
\hline $\begin{array}{l}0 \\
\ddot{0}\end{array}$ & & 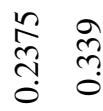 \\
\hline 苞 & 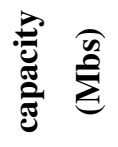 & 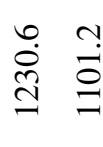 \\
\hline 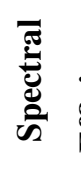 & 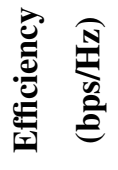 & $\stackrel{\infty}{\stackrel{\sim}{\sim}} \underset{-}{\sigma}$ \\
\hline 总 & $\stackrel{\overrightarrow{0}}{\frac{\hat{n}}{2}}$ & 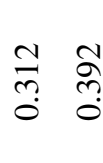 \\
\hline $\overrightarrow{\bar{E}}$ & 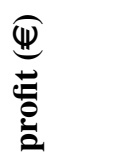 & 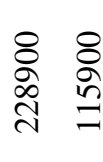 \\
\hline 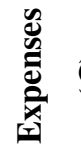 & 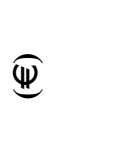 & 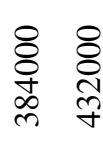 \\
\hline 离 & $\underbrace{2}$ & $\begin{array}{ll}\dot{b} & \stackrel{n}{+} \\
\vec{j}\end{array}$ \\
\hline 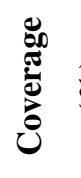 & कृ & 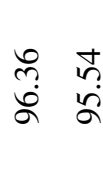 \\
\hline 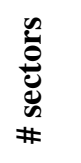 & & $\stackrel{\infty}{+}$ \\
\hline 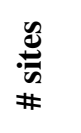 & & $\infty \quad a$ \\
\hline $\bar{\Xi}$ & & $\frac{n}{i} \frac{0}{i}$ \\
\hline
\end{tabular}




\begin{tabular}{|c|c|c|c|}
\hline 苞 & & $\begin{array}{ll}\infty & \tilde{N} \\
\stackrel{0}{0} & \infty \\
\stackrel{0}{0} & \stackrel{0}{0}\end{array}$ & 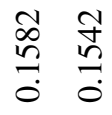 \\
\hline & 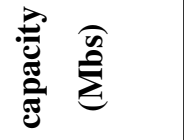 & $\begin{array}{ll}0 & + \\
\dot{\nabla} & \stackrel{8}{0} \\
\stackrel{0}{=}\end{array}$ & $\begin{array}{ll}\infty & \infty \\
\stackrel{\infty}{\infty} & \stackrel{0}{=} \\
= & \end{array}$ \\
\hline 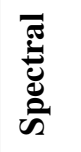 & 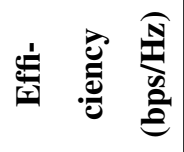 & $\exists \stackrel{\curvearrowleft}{=}$ & $\exists$ \\
\hline$\underset{\mathscr{E}}{\mathscr{D}}$ & 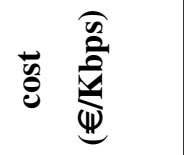 & 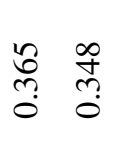 & 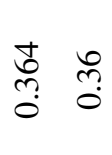 \\
\hline $\overrightarrow{\bar{E}}$ & $\underset{0}{\mathbb{2}}$ & \begin{tabular}{ll}
8 & 8 \\
\multirow{+}{*}{} & $\infty$ \\
ले & 0 \\
\end{tabular} & 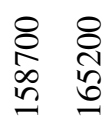 \\
\hline 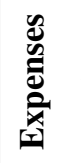 & $\mathbb{\Psi}$ & 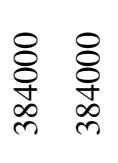 & 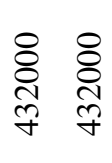 \\
\hline 菢 & $\widehat{8}$ & 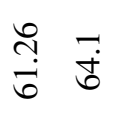 & $\begin{array}{ll} \pm & a \\
0 & 0 \\
0\end{array}$ \\
\hline 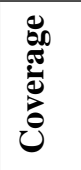 & $\widehat{\theta}$ & 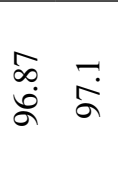 & 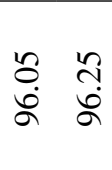 \\
\hline 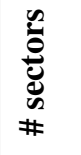 & & $\stackrel{\infty}{+} \stackrel{\infty}{+}$ & in in \\
\hline 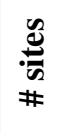 & & $\infty \quad \infty$ & $a \quad a$ \\
\hline 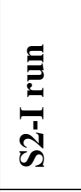 & & 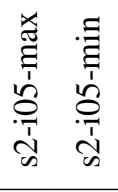 & 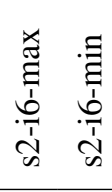 \\
\hline 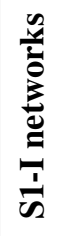 & & $\frac{\mathscr{c}}{\frac{1}{n}}$ & $\frac{9}{10}$ \\
\hline
\end{tabular}




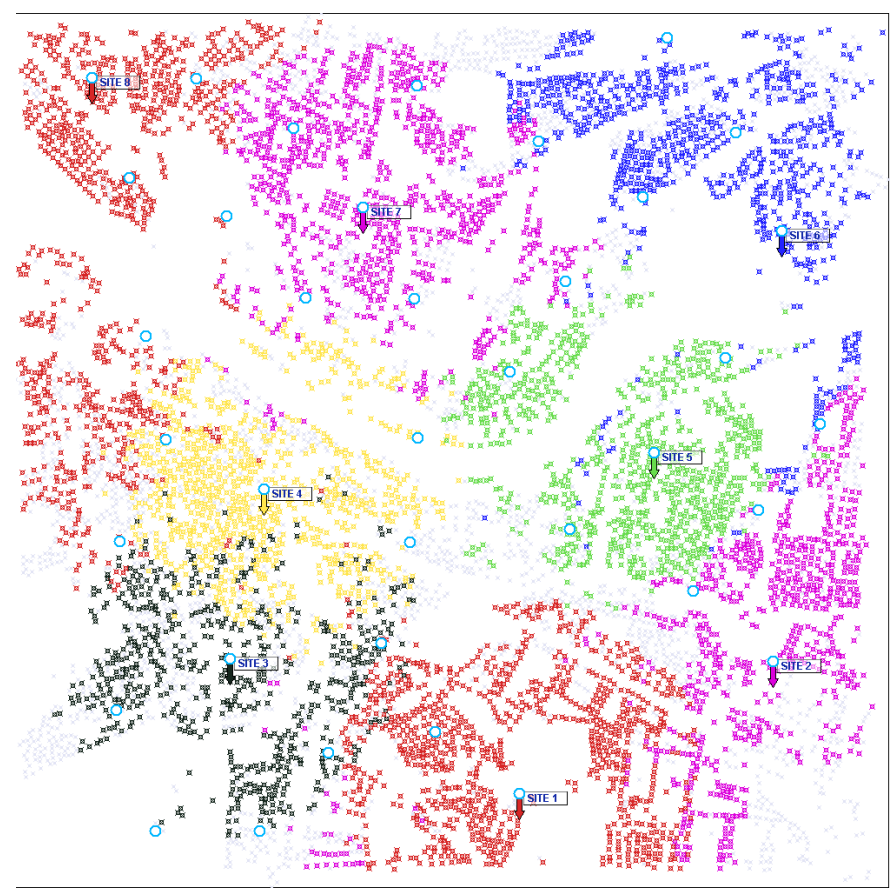

Figure 7.17: Stage 1 network plan - s1-i05

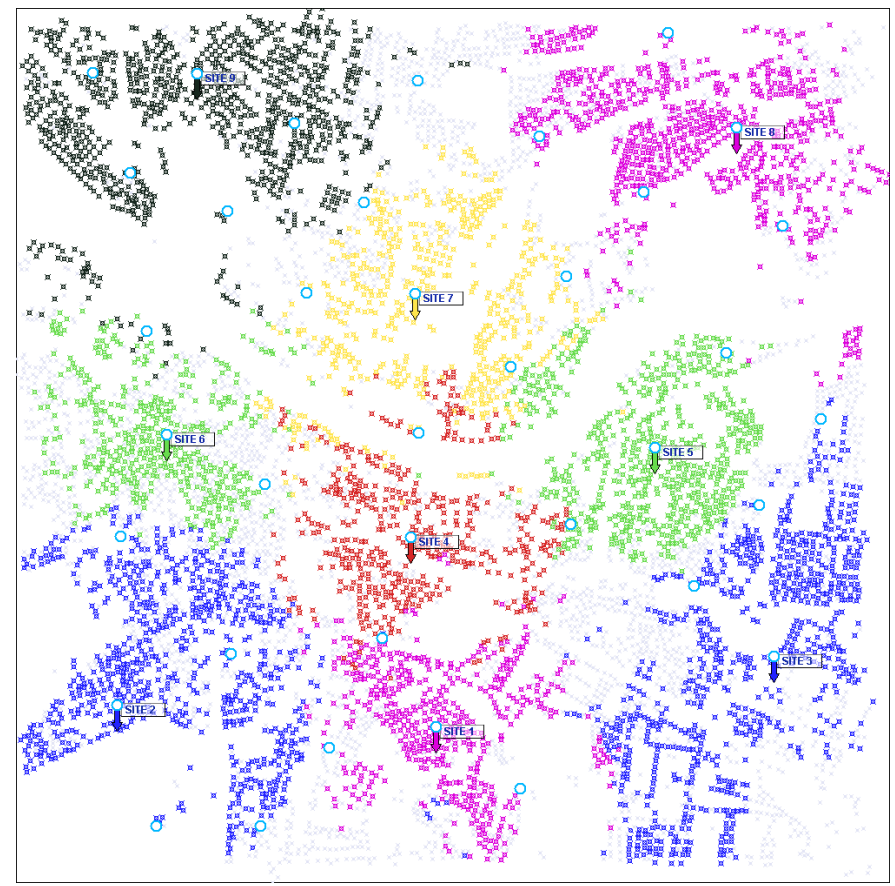

Figure 7.18: Stage 1 network plan - s1-i6 


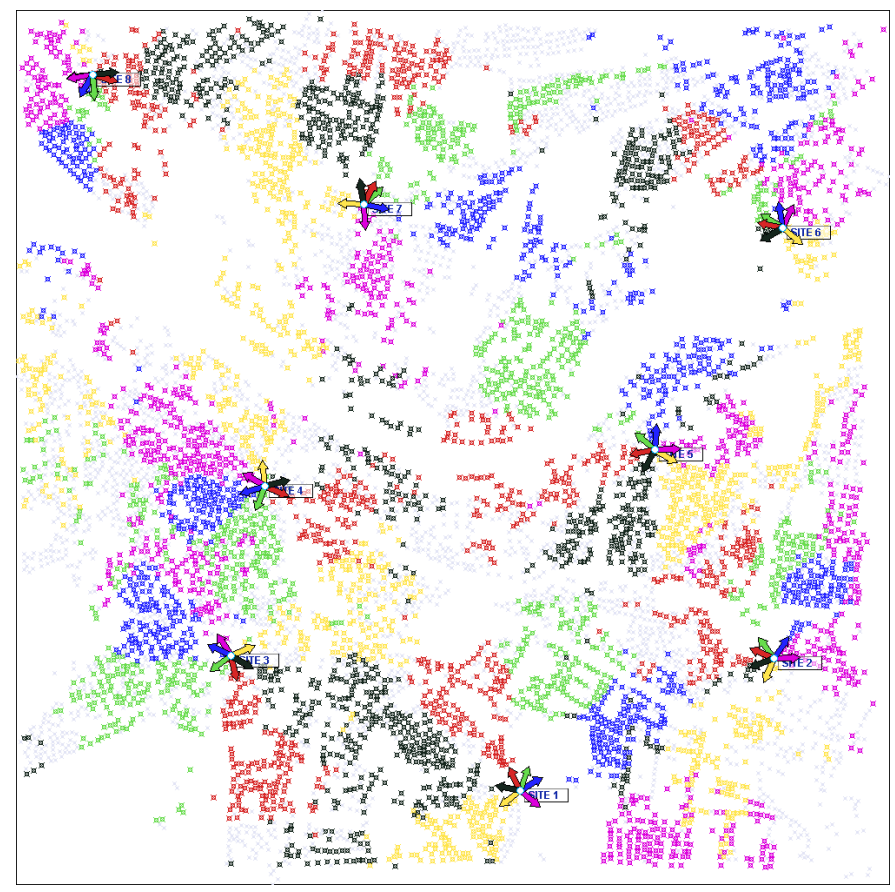

Figure 7.19: Stage 2 network plan - s2-i05

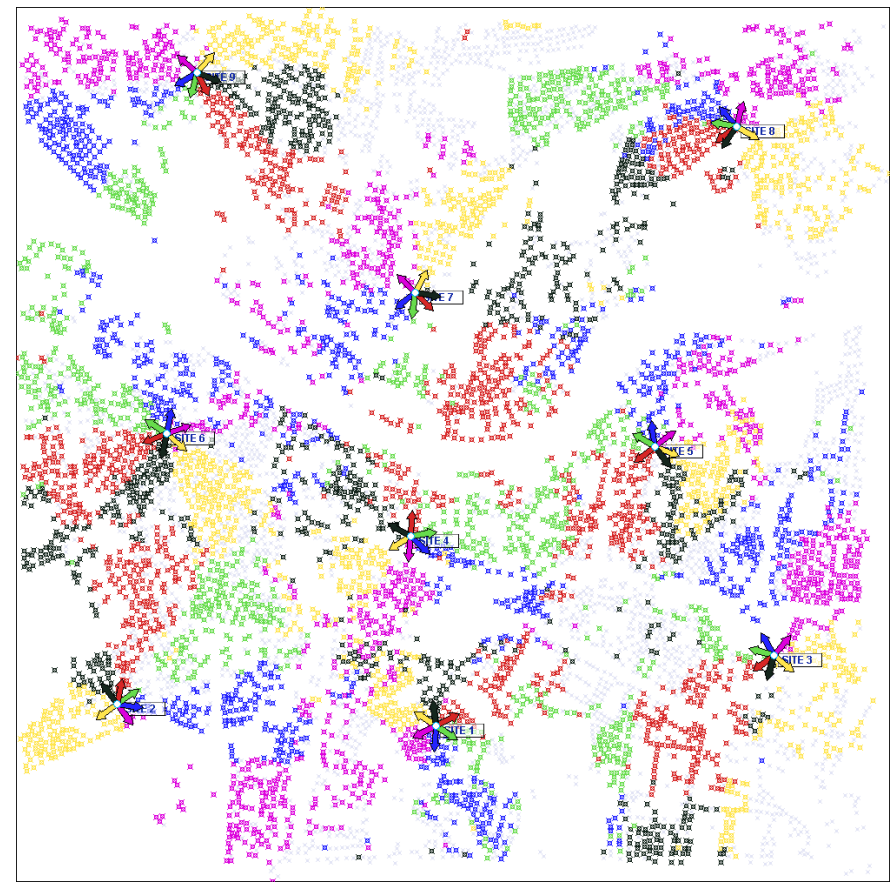

Figure 7.20: Stage 2 network plan - s2-i6 


\begin{tabular}{|c|c|c|c|c|}
\hline S1-I networks & S2-I networks & S2-I stats & S2-I score & S2-I service gain \\
\hline \multirow{4}{*}{ s1-i05 } & \multirow{4}{*}{ s2-i05 } & $\max$ & 0.1968 & \multirow{3}{*}{$-12.14 \%$} \\
\hline & & mean & 0.1879 & \\
\hline & & $\min$ & 0.1823 & \\
\hline & & \multicolumn{3}{|c|}{ std dev: $0.005 ; C V: 2.66 \%$} \\
\hline \multirow{4}{*}{ s1-i6 } & \multirow{4}{*}{ s2-i6 } & $\max$ & 0.1582 & \multirow{3}{*}{$8.49 \%$} \\
\hline & & mean & 0.1561 & \\
\hline & & $\min$ & 0.1542 & \\
\hline & & \multicolumn{3}{|c|}{ std dev: $0.00144 ; C V: 0.92 \%$} \\
\hline
\end{tabular}

Table 7.19: Stage 2 statistical analysis of generated networks after Stage 1 with different interference settings. 


\subsection{Network planning employing standard approach}

The traditional approach in WiMAX network planning presented in the literature is often oversimplified [46, 70, 75, 119, 131, 100]. The main goal of the approaches is facility location where the aim is to position 3-sectored base stations without consideration of azimuth, tilting, or changing antenna radiation patterns, but considering power allocation. This also does not include realistic SINR evaluation, the use of AMC, consideration of permutation schemes, slot or PermBase allocation. At best some authors propose to tackle the facility location problem together with a resource allocation problem simultaneously, however most of the base station configurations are omitted and once again the 3-sectored structure of the BS is used [18]. This significantly simplifies the overall network planning problem which is not suitable in practical cases. In order to underline the benefits, efficiency and practicality of our framework the standard approach was simulated using 3-sectored base stations. Moreover, as is widely published we have fixed the azimuths of sectors within the base stations, but allowed the algorithm to change the tilting and patterns of antennas. Initially, 35 network plans were generated using the Stage 1 procedure. These networks are presented in Tables 7.20 and 7.21, and 5 were selected for subsequent improvement, Table 7.22. As it can be seen the algorithm has generated a wide range of different scenarios with the number of active site locations varying between 12 and 16. However, the most notable metric, profitability, indicates that the use of 3-sectored base stations is much less financially viable, as the cost of purchasing and maintaining the sites greatly exceeds the costs of the base stations. Therefore, most of the generated network plans introduce losses in the first financial year. The service level of the selected network plans is also considerably lower compared to the results given in Section 7.2 due to the fact that the antennas cannot be rotated in the horizontal plane and are unable to efficiently service the surrounding users. The graphical representation of the selected networks can be found on Figures $7.21-7.25$

Stage 2 in this experiment was simplified by sectorizing omni base stations with de- 


\begin{tabular}{|c|c|}
\hline 总 & 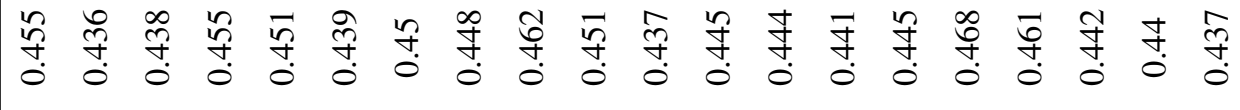 \\
\hline इ & 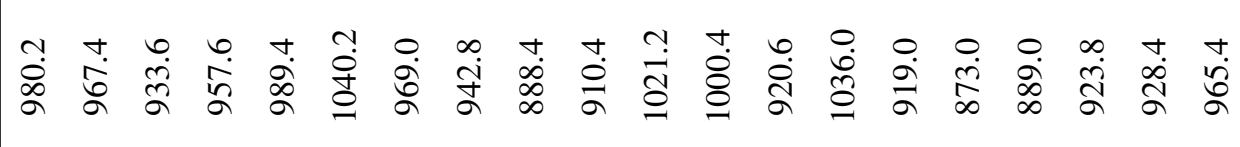 \\
\hline 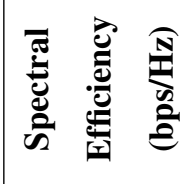 & 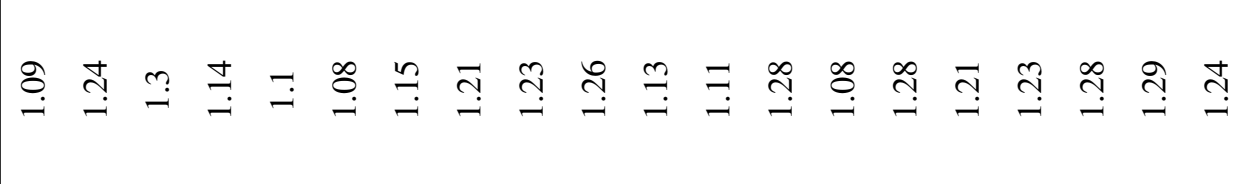 \\
\hline 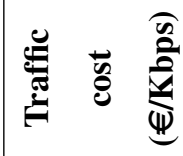 & 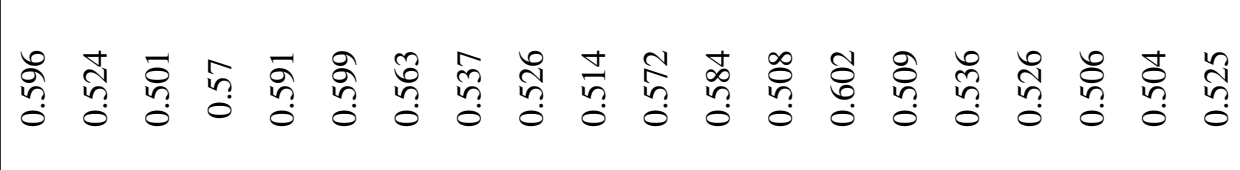 \\
\hline 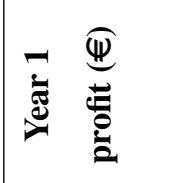 & 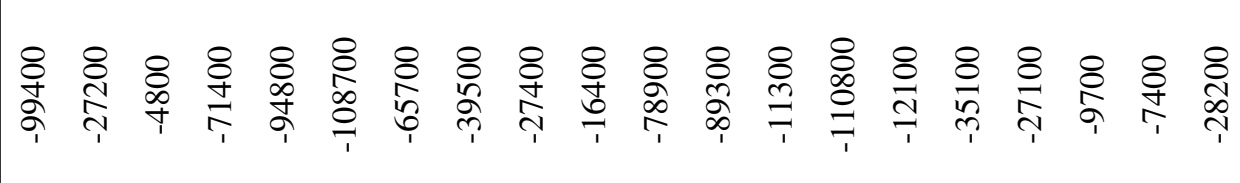 \\
\hline 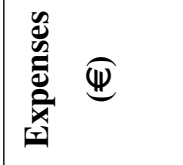 & 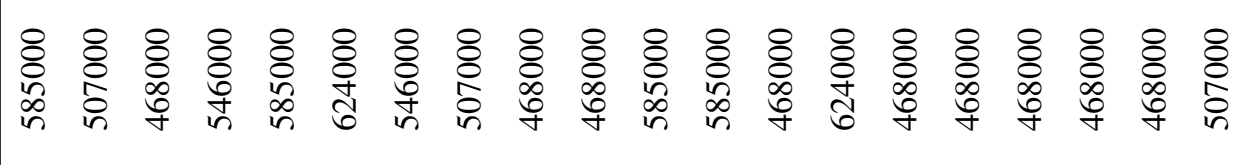 \\
\hline 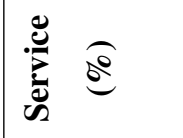 & 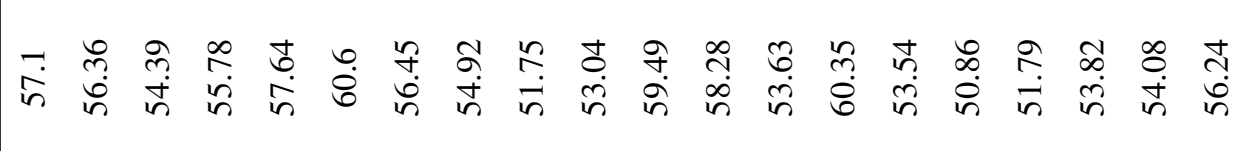 \\
\hline 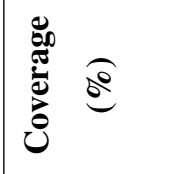 & 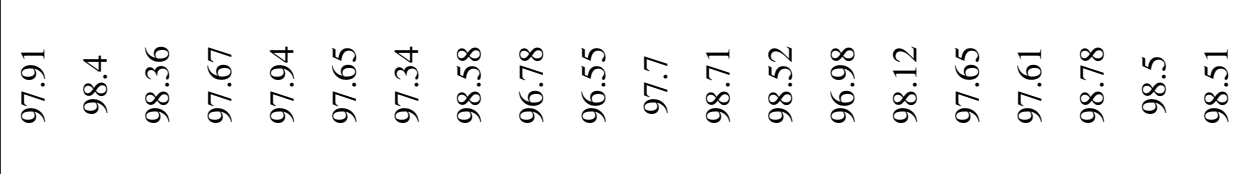 \\
\hline 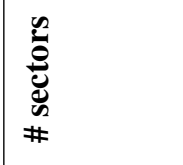 & 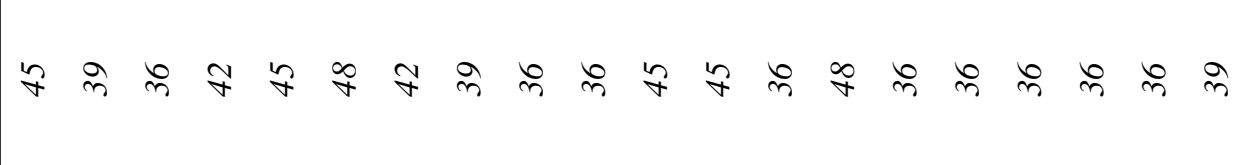 \\
\hline 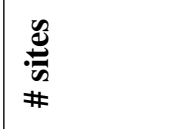 & 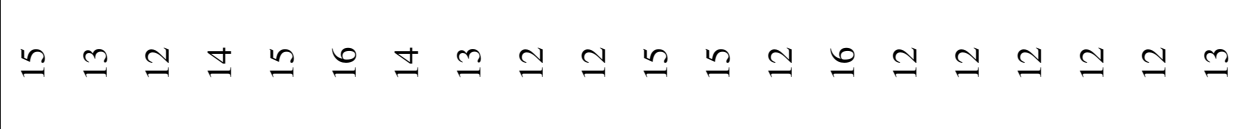 \\
\hline$\underline{\underline{z}}$ & 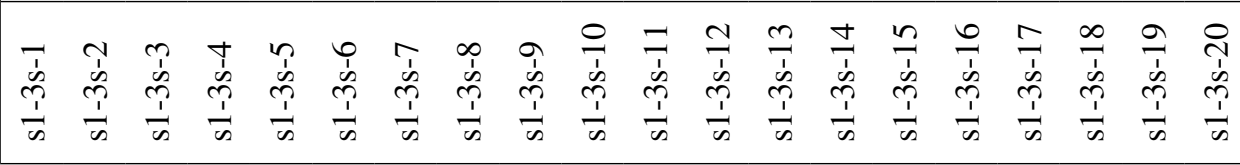 \\
\hline
\end{tabular}




\begin{tabular}{|c|c|c|c|c|c|c|c|c|c|c|c|c|c|c|c|c|c|}
\hline $\begin{array}{l}\stackrel{0}{\breve{U}} \\
\ddot{n}\end{array}$ & & $\begin{array}{l}\stackrel{0}{\circ} \\
\stackrel{7}{0}\end{array}$ & $\underset{+}{\stackrel{J}{*}}$ & 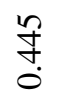 & $\underset{+}{\stackrel{f}{+}}$ & 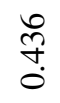 & $\underset{0}{\stackrel{f}{+}}$ & $\stackrel{\tilde{m}}{0}$ & 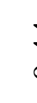 & $\begin{array}{l} \pm \\
0 \\
\end{array}$ & 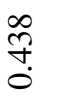 & $\stackrel{?}{\stackrel{f}{*}}$ & $\begin{array}{l}\vec{n} \\
\stackrel{0}{0}\end{array}$ & $\stackrel{\text { fof }}{\circ}$ & $\begin{array}{l}0 \\
0 \\
0 \\
0\end{array}$ & 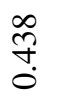 & $\stackrel{m}{\stackrel{?}{0}}$ \\
\hline बूँّ & 㝕 & ڤ̊. & $\begin{array}{l}\text { : } \\
\text { ఏू. }\end{array}$ & $\stackrel{\vec{\sigma}}{\vec{\sigma}}$ & $\frac{\pi}{\infty}$ & $\stackrel{\circ}{\stackrel{\sigma}{\sigma}}$ & $\begin{array}{l}\infty \\
\stackrel{\mathscr{d}}{\sigma}\end{array}$ & $\begin{array}{l}\text { ปे } \\
\text { ¿े }\end{array}$ & & $\frac{0}{0}$ & $\begin{array}{c}\text { ले } \\
\text { ă }\end{array}$ & 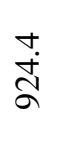 & $\stackrel{\infty}{\bar{\sigma}}$ & $\begin{array}{l}0 \\
\infty \\
\infty \\
0\end{array}$ & $\begin{array}{l}0 \\
\dot{+} \\
\infty \\
\infty\end{array}$ & $\begin{array}{l}\text { તี } \\
\text { నू }\end{array}$ & $\begin{array}{l}0 \\
\stackrel{J}{*} \\
0\end{array}$ \\
\hline $\begin{array}{l}\overline{\tilde{E}} \\
\text { की } \\
\text { की }\end{array}$ & 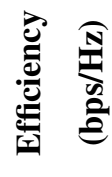 & $\stackrel{g}{0}$ & $\stackrel{\infty}{\stackrel{\infty}{\longrightarrow}}$ & $\stackrel{\infty}{\stackrel{\sim}{\hookrightarrow}}$ & $\stackrel{\infty}{\stackrel{\infty}{\hookrightarrow}}$ & $\stackrel{n}{\longrightarrow}$ & ֻُ & 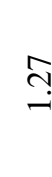 & & $\stackrel{\overbrace{}}{\tilde{c}}$ & $\stackrel{n}{\longrightarrow}$ & 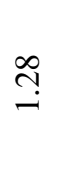 & 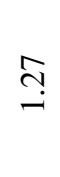 & $\stackrel{n}{\leftrightarrows}$ & 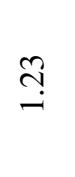 & $\stackrel{\text { సે }}{=}$ & $\bar{m}$ \\
\hline 这 & 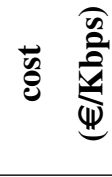 & م̂ñ & $\begin{array}{l}\infty \\
\stackrel{n}{n} \\
0\end{array}$ & $\begin{array}{l}\text { oे } \\
\text { n̊. }\end{array}$ & $\begin{array}{l}\text { oे } \\
\text { n̊. }\end{array}$ & 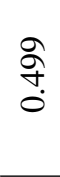 & $\begin{array}{l}\infty \\
\stackrel{0}{0} \\
0 \\
0\end{array}$ & तี & & $\begin{array}{c}\hat{\imath} \\
\tilde{0}\end{array}$ & $\begin{array}{l}\overline{0} \\
\text { nִ } \\
0\end{array}$ & 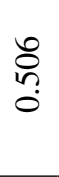 & $\frac{m}{n}$ & $\begin{array}{l}\tilde{0} \\
\stackrel{n}{0}\end{array}$ & ते & $\begin{array}{l}\text { ô } \\
\text { nุ. } \\
0\end{array}$ & $\stackrel{n}{+}$ \\
\hline $\overrightarrow{\bar{z}}$ & $\begin{array}{l}\mathbb{\Psi} \\
\stackrel{\vec{E}}{0} \\
\vdots\end{array}$ & $\begin{array}{l}8 \\
0 \\
0 \\
0 \\
1\end{array}$ & $\begin{array}{l}\stackrel{8}{0} \\
\underset{1}{=}\end{array}$ & 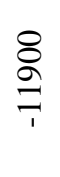 & 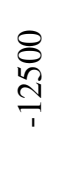 & $\frac{8}{r}$ & $\underset{7}{\stackrel{8}{\mathcal{D}}}$ & 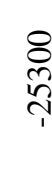 & & 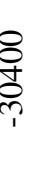 & 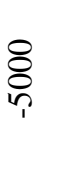 & \& & $\underset{\frac{8}{2}}{\frac{8}{1}}$ & \begin{tabular}{l}
8 \\
\multirow{2}{0}{} \\
1
\end{tabular} & $\begin{array}{l}\stackrel{8}{0} \\
\text { ָิ }\end{array}$ & & ஓ \\
\hline 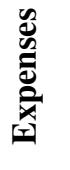 & $\mathbb{\Psi}$ & $\begin{array}{l}8 \\
8 \\
0 \\
\infty \\
\infty \\
n\end{array}$ & \begin{tabular}{l}
8 \\
8 \\
$\infty$ \\
\hdashline \\
\hdashline
\end{tabular} & \begin{tabular}{l}
8 \\
8 \\
$\infty$ \\
$\&$ \\
\multirow{o}{*}{}
\end{tabular} & \begin{tabular}{l}
8 \\
8 \\
$\infty$ \\
$\infty$ \\
\multirow{+}{*}{}
\end{tabular} & $\begin{array}{l}8 \\
8 \\
\infty \\
0 \\
0 \\
+\end{array}$ & $\begin{array}{l}8 \\
\delta \\
\infty \\
0 \\
o \\
+\end{array}$ & $\begin{array}{l}8 \\
8 \\
+ \\
\stackrel{8}{0}\end{array}$ & & $\begin{array}{l}8 \\
8 \\
0 \\
0 \\
n\end{array}$ & $\begin{array}{l}8 \\
8 \\
\infty \\
o \\
o \\
+\end{array}$ & $\begin{array}{l}8 \\
8 \\
\infty \\
o \\
o \\
+\end{array}$ & $\begin{array}{l}8 \\
8 \\
\infty \\
8 \\
8\end{array}$ & $\begin{array}{l}8 \\
8 \\
0 \\
0 \\
i\end{array}$ & $\begin{array}{l}8 \\
8 \\
8 \\
8 \\
8 \\
+\end{array}$ & \begin{tabular}{l}
8 \\
8 \\
$\infty$ \\
\hdashline \\
\hdashline
\end{tabular} & 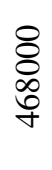 \\
\hline 离 & es & 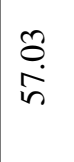 & $\begin{array}{l}\hat{\beta} \\
\text { in }\end{array}$ & 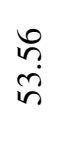 & 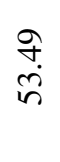 & $\begin{array}{l}\stackrel{\infty}{+} \\
\stackrel{+}{n}\end{array}$ & $\begin{array}{l}\dot{t} \\
\text { ñ }\end{array}$ & त̂. & & 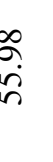 & $\begin{array}{l}\text { D̃ } \\
\stackrel{+}{n} \\
\text { in }\end{array}$ & $\begin{array}{l}\infty \\
\infty \\
n \\
n\end{array}$ & $\frac{7}{i n}$ & $\begin{array}{l}\stackrel{\text { fे }}{\bullet} \\
\stackrel{n}{n}\end{array}$ & $\stackrel{n}{n}$ & $\begin{array}{l}\vec{m} \\
\stackrel{+}{n}\end{array}$ & $\begin{array}{l}0 \\
\dot{n} \\
i n\end{array}$ \\
\hline 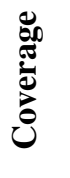 & $\widehat{\theta}$ & $\frac{9}{5}$ & 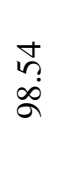 & $\begin{array}{l}n \\
\infty \\
\infty\end{array}$ & 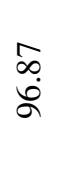 & $\begin{array}{l}n \\
\mathfrak{b} \\
\infty \\
\infty\end{array}$ & 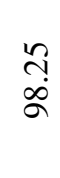 & $\begin{array}{l}\infty \\
\vdots \\
\vdots \\
\sigma\end{array}$ & & 官 & $\begin{array}{l}\hat{n} \\
\infty \\
\infty\end{array}$ & $\begin{array}{l}\text { J } \\
\infty \\
\infty\end{array}$ & $\begin{array}{l}\hat{\alpha} \\
\text { na }\end{array}$ & \begin{tabular}{l}
$\stackrel{n}{f}$ \\
\multirow{a}{a}{}
\end{tabular} & 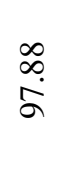 & $\begin{array}{l}\stackrel{\infty}{\infty} \\
\stackrel{\infty}{ }\end{array}$ & $\stackrel{0}{\infty}$ \\
\hline 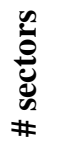 & & $\stackrel{n}{7}$ & $\stackrel{i}{n}$ & p & D & r & m & $\stackrel{\infty}{m}$ & & ले & r & r & లి & $\approx$ & ln & in & r \\
\hline 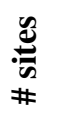 & & $\stackrel{2}{2}$ & $\simeq$ & $\simeq$ & $\simeq$ & $\simeq$ & $\simeq$ & 2 & & 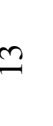 & $\simeq$ & $\simeq$ & $\simeq$ & $\Xi$ & $\simeq$ & $\simeq$ & $\simeq$ \\
\hline 志 & & $\begin{array}{l}\vec{r} \\
\dot{b} \\
\vec{r} \\
\frac{\omega}{n}\end{array}$ & $\begin{array}{l}\tilde{N} \\
\dot{n} \\
\dot{1} \\
\frac{1}{n}\end{array}$ & 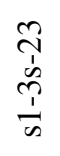 & 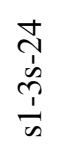 & $\begin{array}{l}\hat{a} \\
\dot{b} \\
\dot{n} \\
\frac{1}{n}\end{array}$ & $\begin{array}{l}\tilde{N} \\
\dot{m} \\
\tilde{c} \\
\dot{\omega}\end{array}$ & $\begin{array}{l}\hat{y} \\
\dot{n} \\
\frac{1}{n}\end{array}$ & & 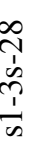 & 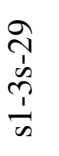 & $\begin{array}{l}\stackrel{p}{\dot{b}} \\
\dot{n} \\
\frac{1}{n}\end{array}$ & $\begin{array}{l}\vec{n} \\
\dot{n} \\
\frac{n}{n}\end{array}$ & $\begin{array}{l}\text { c) } \\
\dot{b} \\
\frac{1}{n} \\
\dot{n}\end{array}$ & $\begin{array}{l}m \\
\dot{n} \\
\dot{n} \\
\dot{n}\end{array}$ & $\begin{array}{l}\dot{m} \\
\dot{b} \\
\dot{m} \\
\dot{n}\end{array}$ & $\begin{array}{l}\stackrel{n}{b} \\
\dot{m} \\
\dot{m} \\
\dot{n}\end{array}$ \\
\hline
\end{tabular}




\begin{tabular}{|c|c|}
\hline 离 & 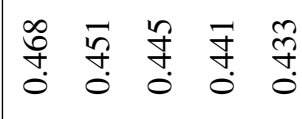 \\
\hline 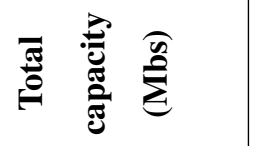 & 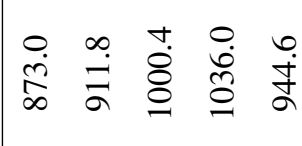 \\
\hline 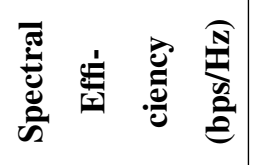 & 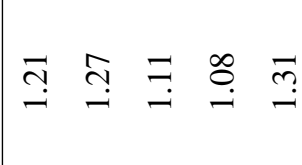 \\
\hline 总 & 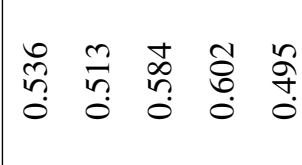 \\
\hline 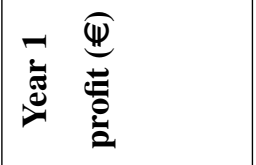 & 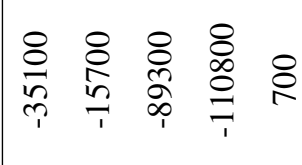 \\
\hline 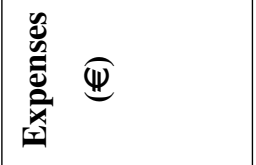 & 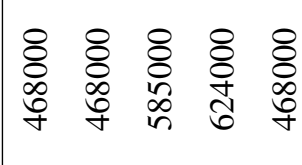 \\
\hline 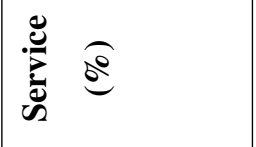 & 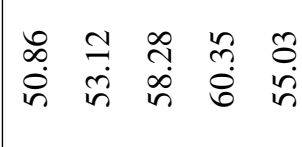 \\
\hline$\widehat{d}$ & 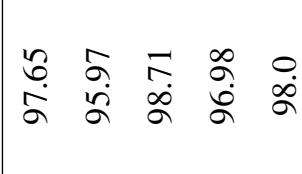 \\
\hline 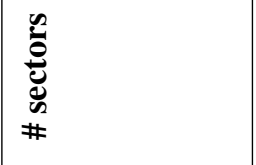 & 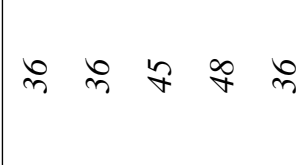 \\
\hline 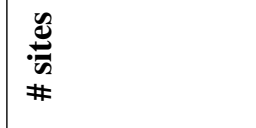 & $\simeq \simeq \cong \simeq \simeq$ \\
\hline 声 & 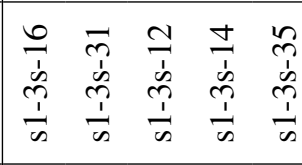 \\
\hline 总 & 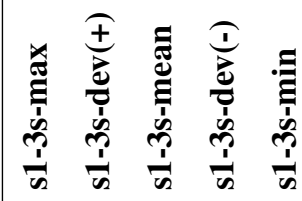 \\
\hline
\end{tabular}




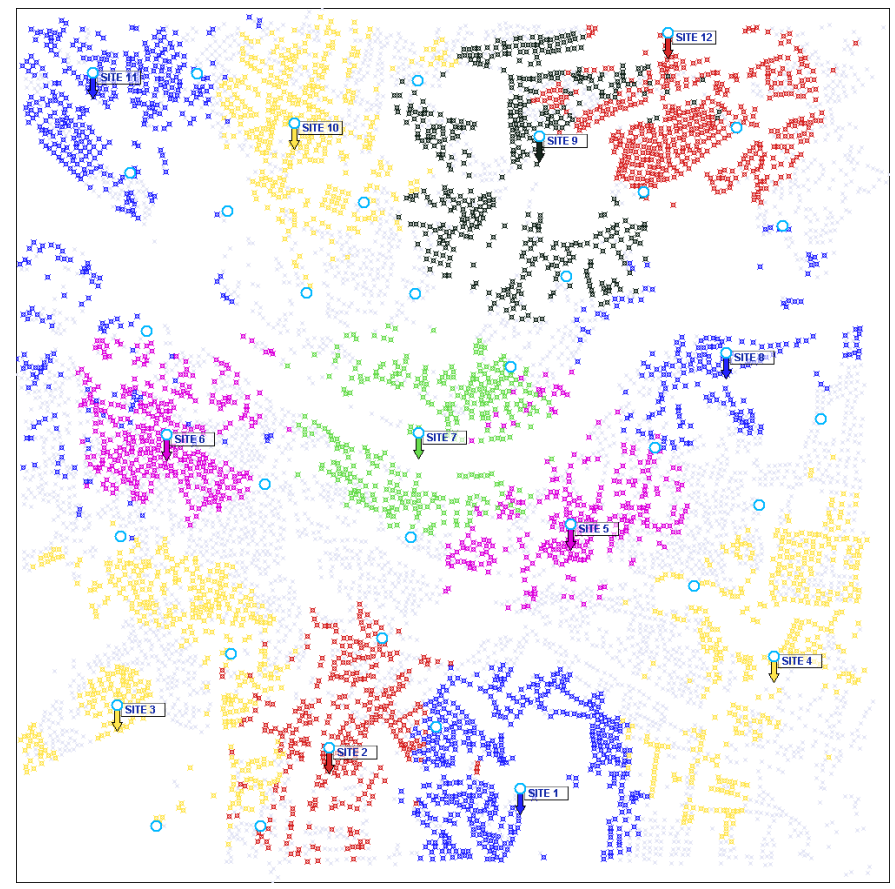

Figure 7.21: Stage 1 standard approach network plan - s1-3s-max

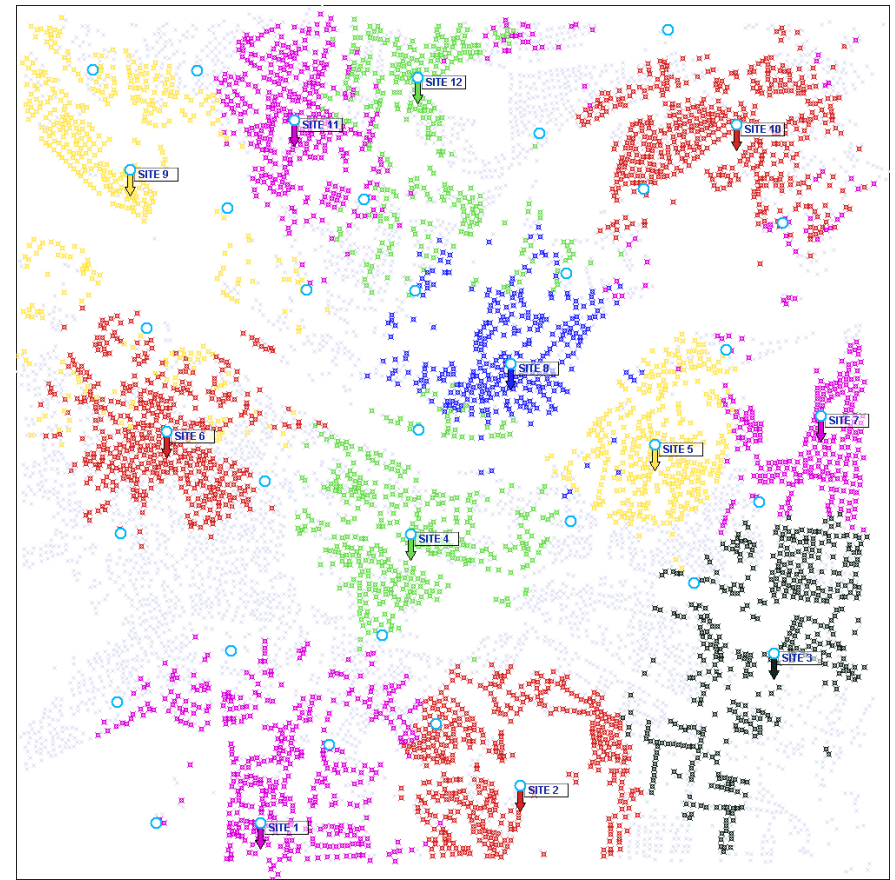

Figure 7.22: Stage 1 standard approach network plan - s1-3s-dev(+) 


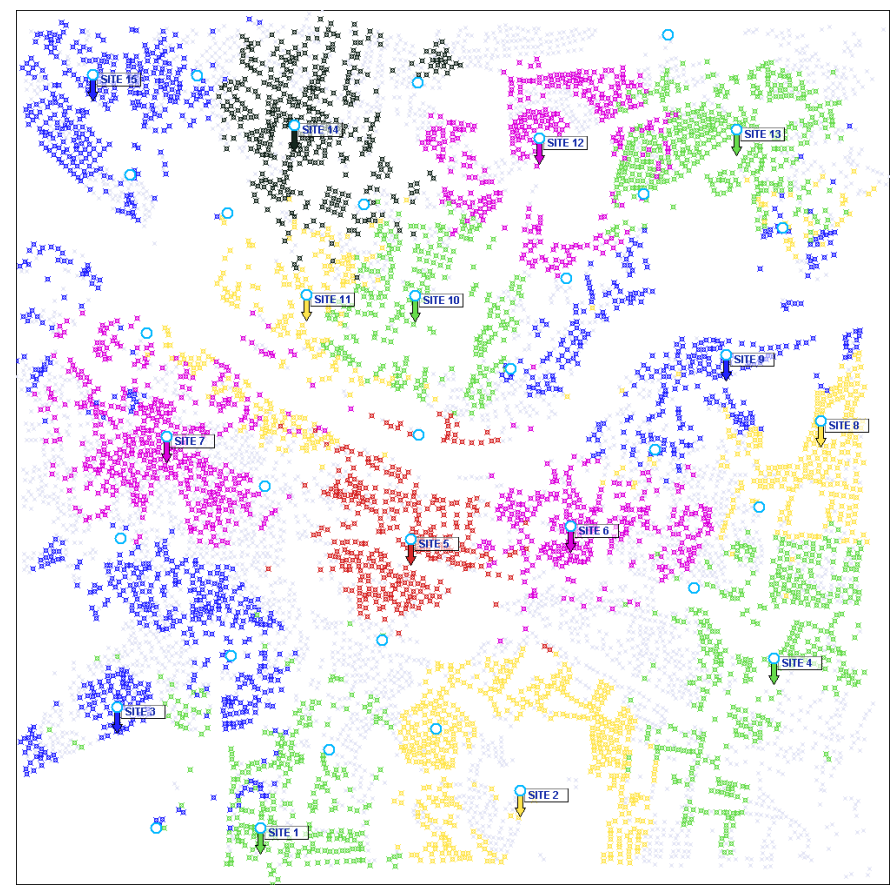

Figure 7.23: Stage 1 standard approach network plan - s1-3s-mean

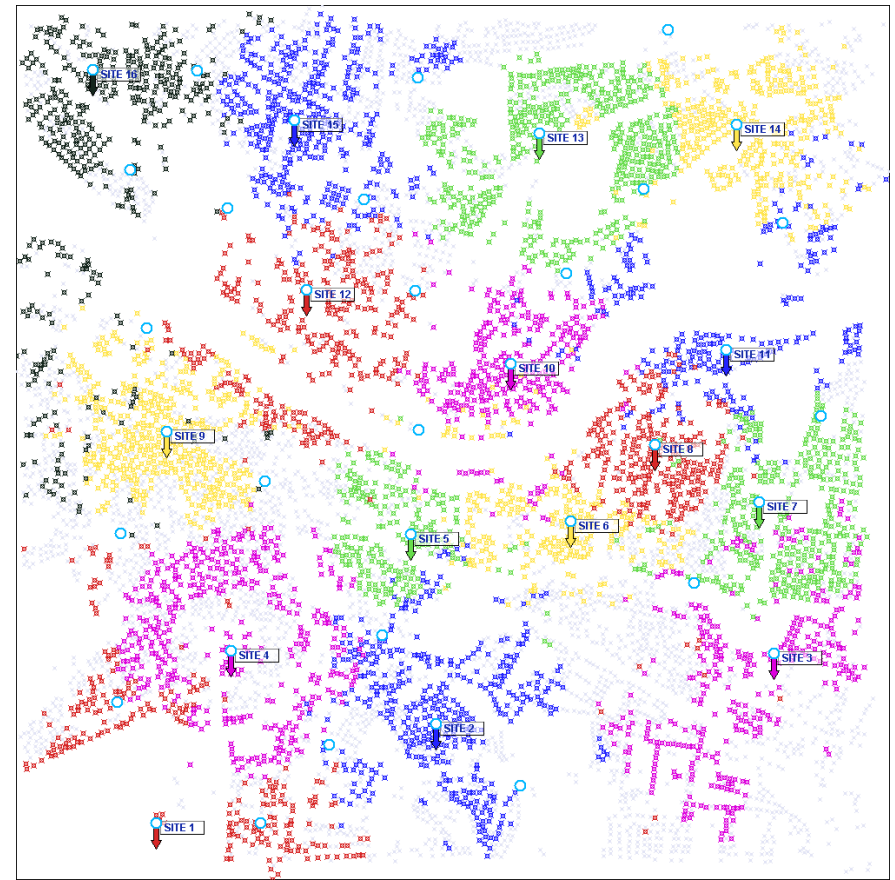

Figure 7.24: Stage 1 standard approach network plan - s1-3s-dev(-) 


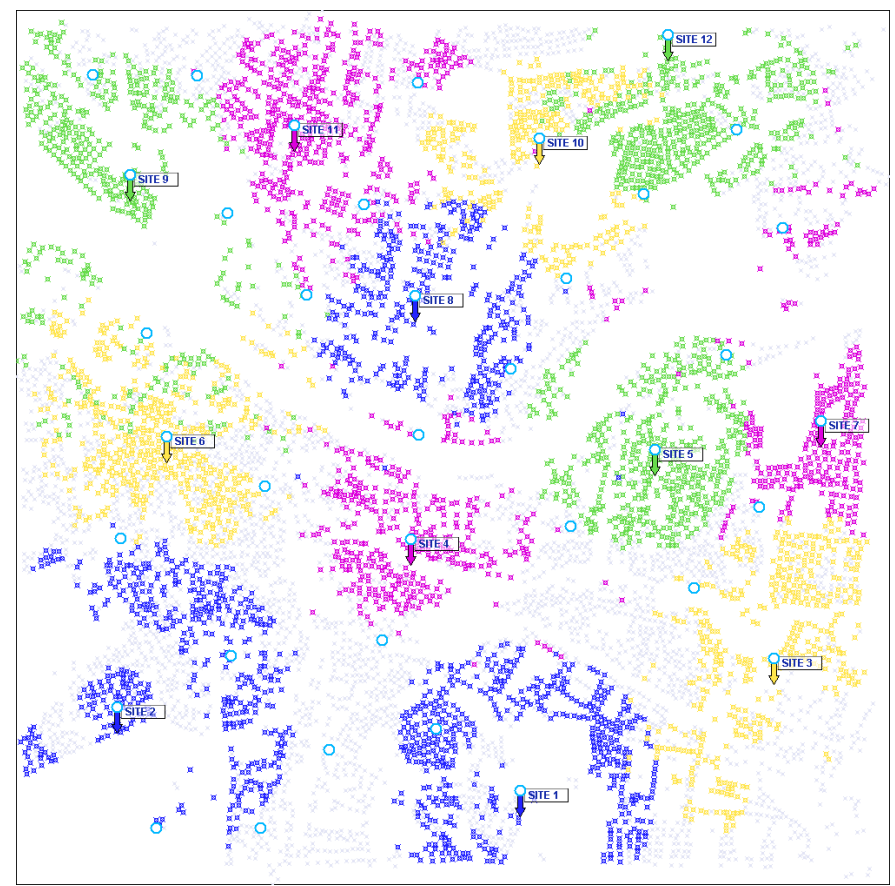

Figure 7.25: Stage 1 standard approach network plan - s1-3s-min 
fault configurations without any modification of antenna type, azimuth, tilting or power level. The sectorized networks are reflected in Table 7.23 where all KPIs are further deteriorated due to the default settings of the equipment. The final stage, which includes modification of power, tilt and antenna type, improves the results over the ones obtained after sectorization in Stage 2, however it fails to achieve the kind of results produced in Stage 1 (Tables 7.24 and 7.25). This can be explained by the elimination of the 'change azimuth' move from the optimization procedure which plays a significant role in achieving effective BS settings for the increase of overall network service. This experiment shows that simplifying the optimization framework with the use of 3 -sectored base stations and minimizing the number of decision variables, in terms of BS modifiers, dramatically reduces the quality of the resulting network designs making their use in practical situations not viable. The graphical representation of the final cell plans is provide on Figures 7.26 7.30. 


\begin{tabular}{|c|c|}
\hline : & 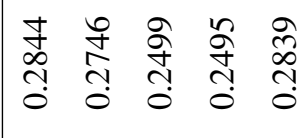 \\
\hline 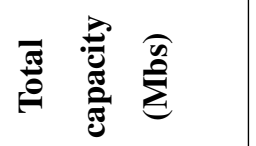 & 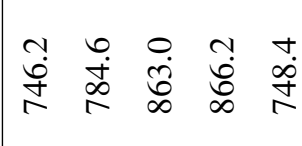 \\
\hline 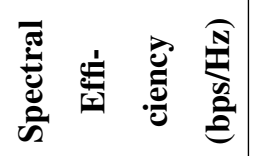 & 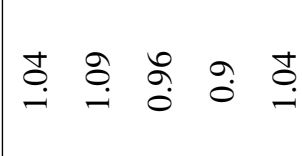 \\
\hline 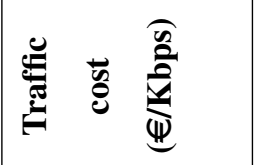 & 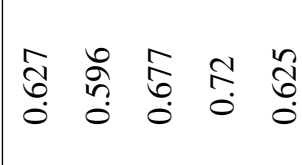 \\
\hline 可 & 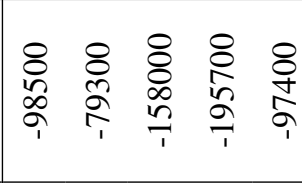 \\
\hline 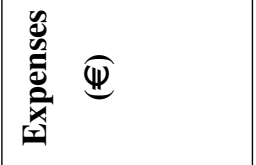 & 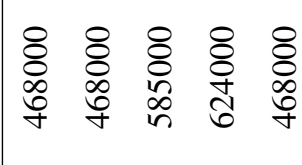 \\
\hline 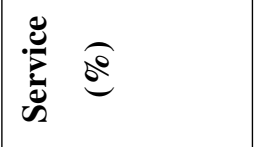 & 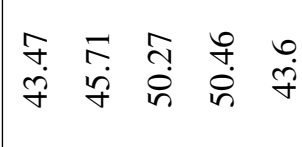 \\
\hline 苞 & 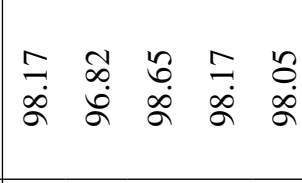 \\
\hline 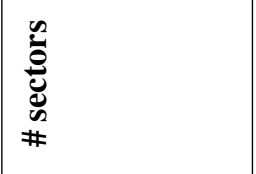 & 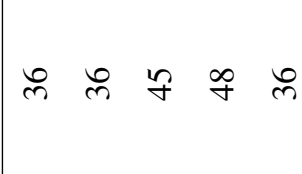 \\
\hline 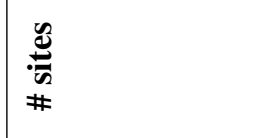 & $\simeq \simeq \cong \simeq$ \\
\hline 政 & 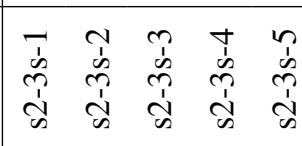 \\
\hline 竞 & 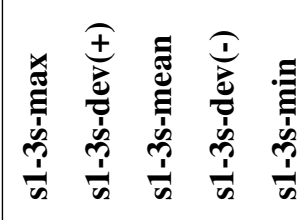 \\
\hline
\end{tabular}




\begin{tabular}{|c|c|c|c|c|c|c|c|c|c|}
\hline ڤ̆ & & & $\begin{array}{ll}\infty & 0 \\
0 & 0 \\
n & 1 \\
0 & 0\end{array}$ & $\stackrel{n}{n}$ & 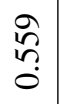 & $\begin{array}{l}\infty \\
n \\
n \\
0\end{array}$ & & 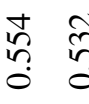 & $\begin{array}{ll}0 & 0 \\
0 & 0 \\
n & 0 \\
0 & 0\end{array}$ \\
\hline 矛 & 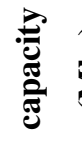 & $\sum_{\hat{\Sigma}}^{\widehat{E}}$ & $\begin{array}{cc} & + \\
\infty & \infty \\
& \infty\end{array}$ & $\stackrel{+}{\stackrel{+}{2}}$ & $\begin{array}{l}\infty \\
\stackrel{\infty}{\infty}\end{array}$ & $\begin{array}{l}0 \\
\dot{\delta} \\
\tilde{\sigma}\end{array}$ & & 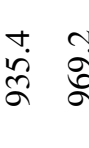 & 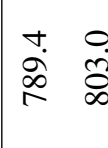 \\
\hline 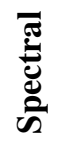 & 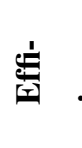 & 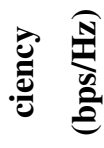 & $\exists \stackrel{0}{=}$ & & $\stackrel{ \pm}{\Xi}$ & $\stackrel{\circ}{-}$ & & $\hat{a}$ & $\exists I$ \\
\hline 思 & $\vec{b}$ & $\frac{0}{\frac{0}{2}}$ & $\begin{array}{ll}\infty & \overline{0} \\
\infty & 0 \\
n & n \\
0 & 0\end{array}$ & $\begin{array}{l}\infty \\
\stackrel{\infty}{0} \\
n\end{array}$ & $n$ & $\begin{array}{l}\text { fo } \\
0 \\
0\end{array}$ & & $\begin{array}{ll}\hat{\delta} & \tilde{z} \\
0 & 0 \\
0 & 0\end{array}$ & 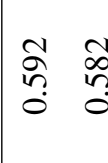 \\
\hline$\dot{\Xi}$ & 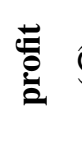 & & 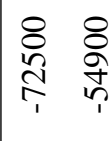 & 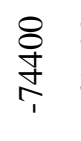 & $\frac{8}{\frac{8}{6}}$ & $\begin{array}{l}\stackrel{8}{\&} \\
\stackrel{1}{*} \\
\stackrel{m}{1}\end{array}$ & & $\begin{array}{ll}8 & 8 \\
\frac{8}{6} & \frac{7}{7} \\
\frac{7}{1} & \frac{1}{1}\end{array}$ & $\mid \begin{array}{ll}8 & 8 \\
0 & 0 \\
0 & 0 \\
1 & 9\end{array}$ \\
\hline 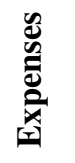 & 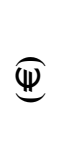 & & \begin{tabular}{ll}
8 & 8 \\
0 & 8 \\
$\infty$ & 0 \\
\hdashline & 0
\end{tabular} & $\begin{array}{l}8 \\
8 \\
0 \\
\infty \\
o\end{array}$ & $\begin{array}{l}8 \\
8 \\
\infty \\
0 \\
0 \\
+\end{array}$ & $\begin{array}{ll}8 & \\
8 & \\
0 & 1 \\
\infty & 0 \\
n & 4\end{array}$ & & 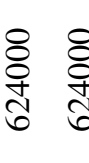 & 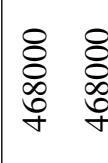 \\
\hline : & 89 & & $\begin{array}{ll}n & n \\
o & n \\
+ & \infty\end{array}$ & 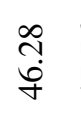 & 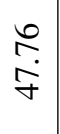 & $\begin{array}{l}\dot{J} \\
i \\
i\end{array}$ & & $\begin{array}{ll}g & 0 \\
+ & + \\
\text { in } & 0\end{array}$ & 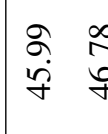 \\
\hline 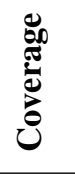 & $\widehat{8}$ & & $\begin{array}{ll}n & \bar{a} \\
\infty & \sigma\end{array}$ & 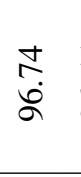 & 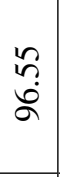 & $\begin{array}{ll}\dot{1} & 0 \\
\infty & \vdots \\
\infty & 0\end{array}$ & & 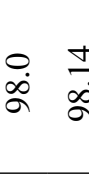 & $\begin{array}{ll}\infty & 5 \\
\hat{a} & 5\end{array}$ \\
\hline 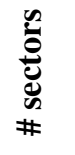 & & & లి & లి & $\stackrel{\sim}{n}$ & r & & $\stackrel{\infty}{+} \stackrel{\infty}{+}$ & $\stackrel{r}{c}$ \\
\hline 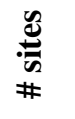 & & & $\simeq \simeq$ & $\simeq$ & $\simeq$ & $\cong$ & & 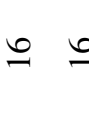 & $\simeq \bumpeq$ \\
\hline$\underset{E}{E}$ & & & 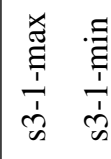 & 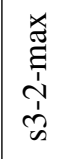 & 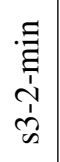 & 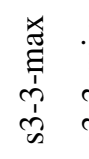 & & 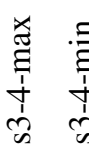 & 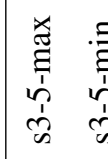 \\
\hline 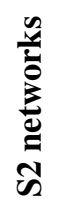 & & & के & ஸे & & $\begin{array}{l}n \\
\dot{n} \\
\tilde{n} \\
\tilde{n}\end{array}$ & & $\begin{array}{l}\dot{b} \\
\tilde{d} \\
\tilde{\omega}\end{array}$ & $\begin{array}{l}n \\
\dot{n} \\
\dot{d} \\
\infty\end{array}$ \\
\hline
\end{tabular}




\begin{tabular}{|c|c|c|c|c|}
\hline S2-3S networks & $\begin{array}{c}\text { S3-3S } \\
\text { networks }\end{array}$ & S3-3S stats & S3-3S score & $\begin{array}{c}\text { S3-3S service } \\
\text { gain }\end{array}$ \\
\hline \multirow{4}{*}{$s 2-3 s-1$} & \multirow{4}{*}{ s3-3s-1 } & $\max$ & 0.5687 & \\
\hline & & mean & 0.5529 & s1: $-5.47 \%$ \\
\hline & & $\min$ & 0.5466 & s2: $10.28 \%$ \\
\hline & & \multicolumn{3}{|c|}{ std dev: $0.00827 ; C V: 2.56 \%$} \\
\hline \multirow{4}{*}{ s2-3s-2 } & \multirow{4}{*}{ s3-3s-2 } & $\max$ & 0.5735 & \\
\hline & & mean & 0.5667 & s1: $-11.43 \%$ \\
\hline & & $\min$ & 0.5593 & s2: $2.93 \%$ \\
\hline & & \multicolumn{3}{|c|}{ std dev: $0.0058 ; C V: 1.02 \%$} \\
\hline \multirow{4}{*}{ s2-3s-3 } & \multirow{4}{*}{ s3-3s-3 } & $\max$ & 0.5586 & \\
\hline & & mean & 0.5441 & s1: $-7.46 \%$ \\
\hline & & $\min$ & 0.5294 & $\mathrm{~s} 2: 7.28 \%$ \\
\hline & & \multicolumn{3}{|c|}{ std dev: $0.00931 ; C V: 1.17 \%$} \\
\hline \multirow{4}{*}{ s2-3s-4 } & \multirow{4}{*}{ s3-3s-4 } & $\max$ & 0.5541 & \\
\hline & & mean & 0.5373 & s1: $-7.71 \%$ \\
\hline & & $\min$ & 0.5321 & s2: $10.38 \%$ \\
\hline & & \multicolumn{3}{|c|}{ std dev: $0.00842 ; C V: 1.57 \%$} \\
\hline \multirow{4}{*}{ s2-3s-5 } & \multirow{4}{*}{ s3-3s-5 } & $\max$ & 0.5664 & \\
\hline & & mean & 0.5658 & s1: $-15.63 \%$ \\
\hline & & $\min$ & 0.5606 & s2: $6.49 \%$ \\
\hline & & \multicolumn{3}{|c|}{ std dev: 0.00206; CV: $3.64 \%$} \\
\hline
\end{tabular}

Table 7.25: Stage 3 statistical analysis of generated networks with standard approach. 


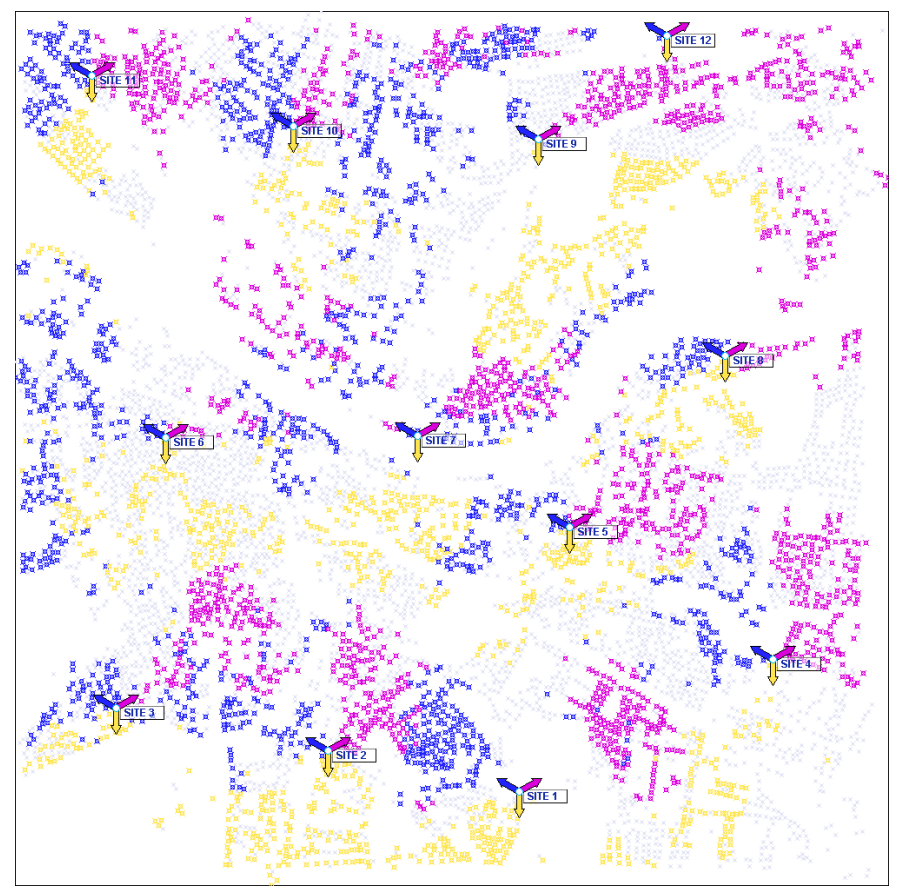

Figure 7.26: Stage 3 standard approach network plan - s3-3s-1 


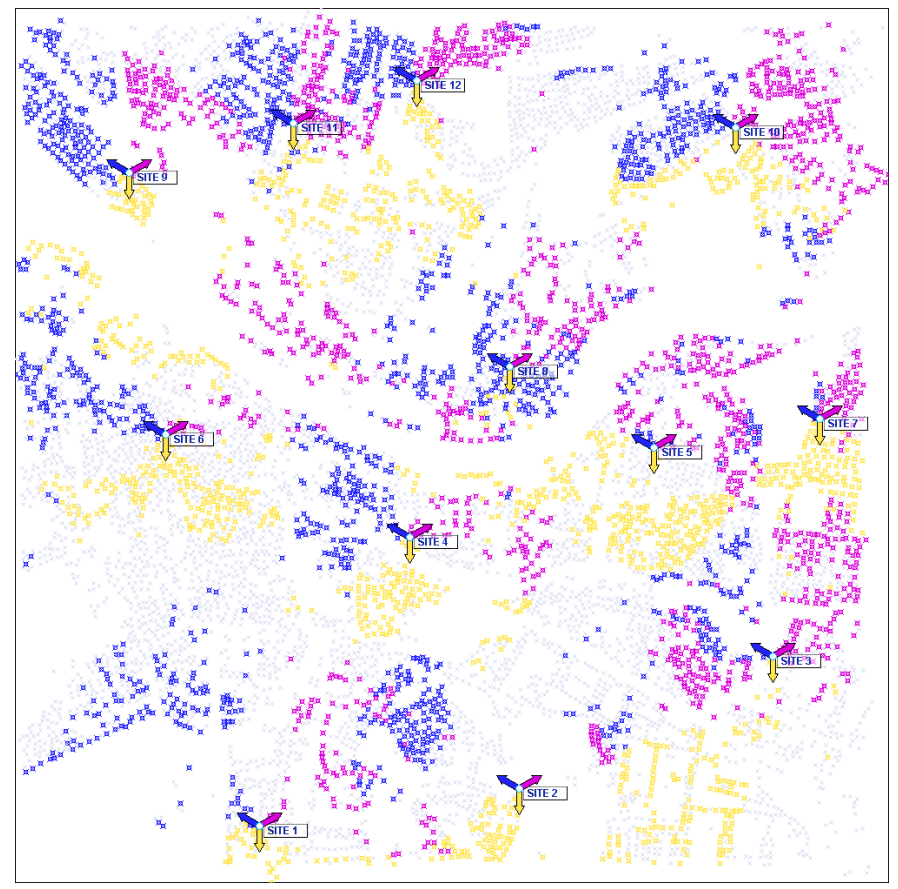

Figure 7.27: Stage 3 standard approach network plan - s3-3s-2

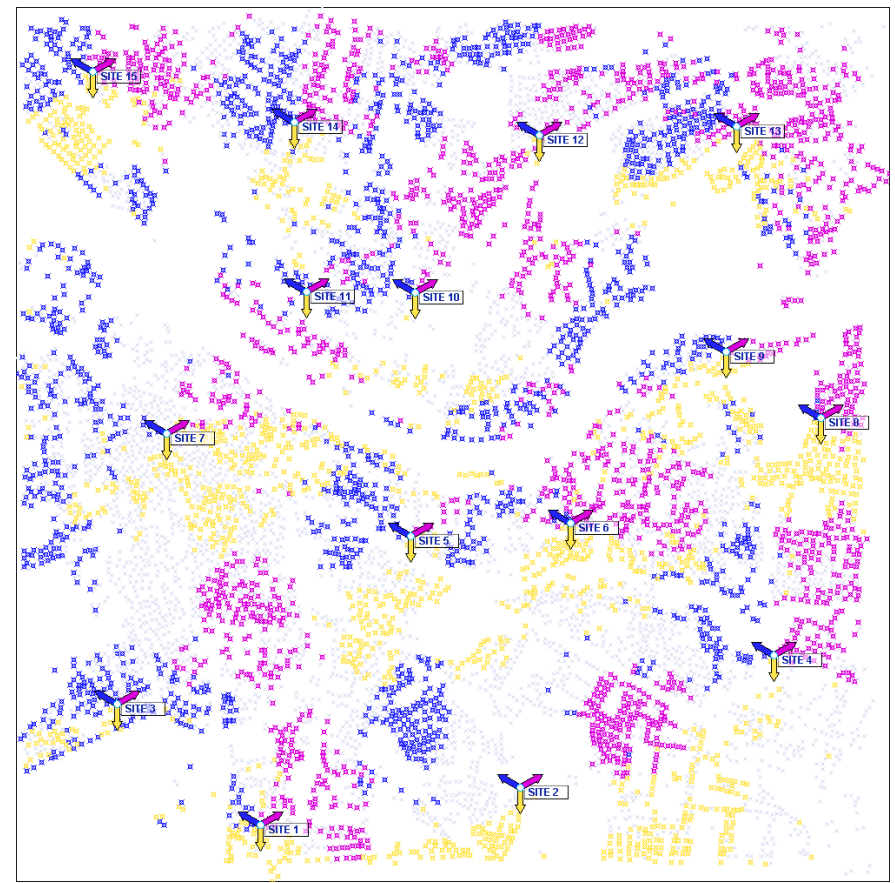

Figure 7.28: Stage 3 standard approach network plan - s3-3s-3 


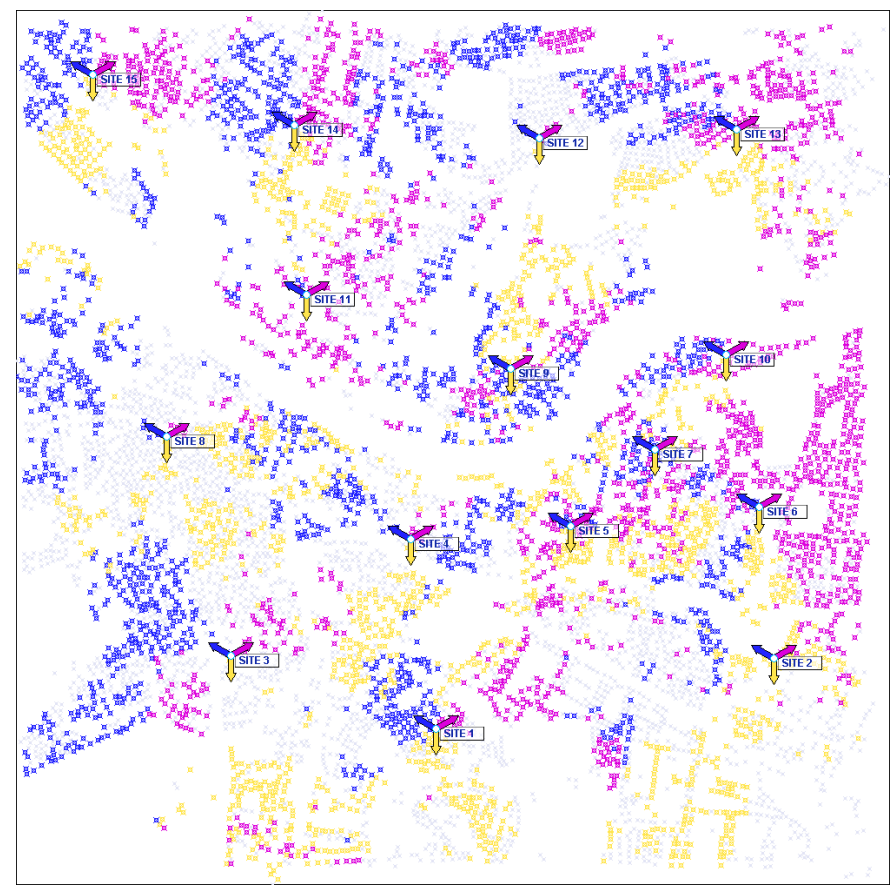

Figure 7.29: Stage 3 standard approach network plan - s3-3s-4

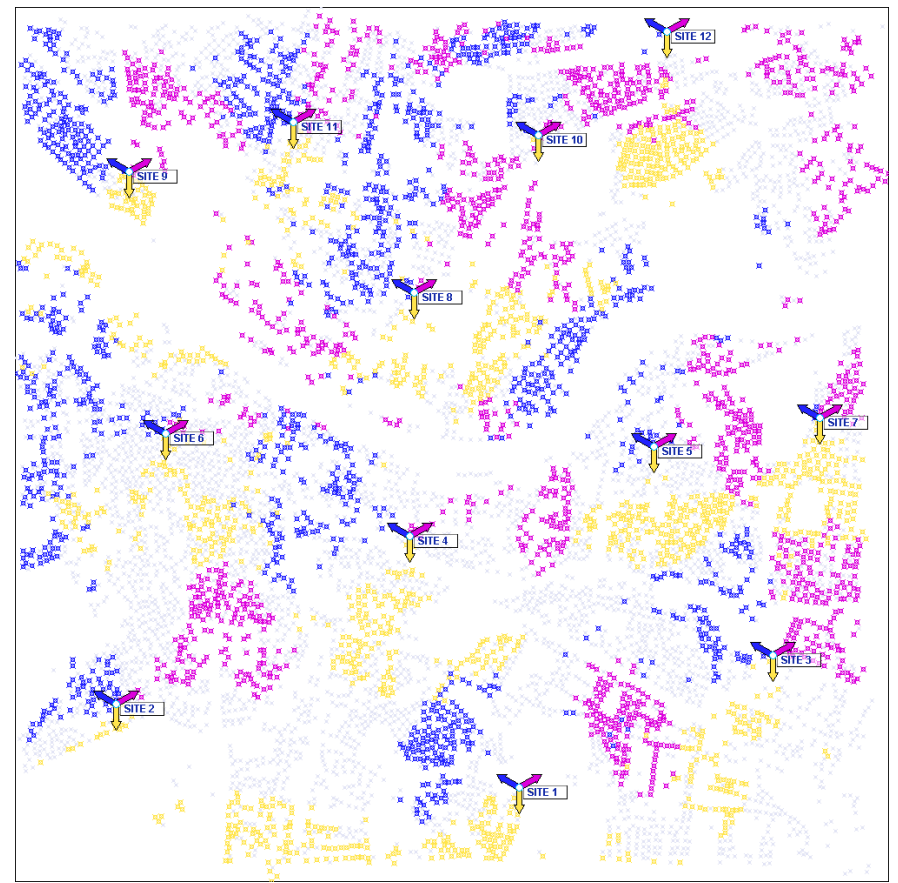

Figure 7.30: Stage 3 standard approach network plan - s3-3s-5 


\subsection{Analysis of the results}

Having generated WiMAX networks employing different planning experiments that cover (i) the proposed optimization framework, (ii) Stage 3 network planning with fixed PermBase in all the network base station sectors, (iii) cells plans with simplified Stage 2 procedure, (iv) initial planning and dimensioning using Stage 1 with different capacity-interference parameters and finally (v) simulating a standard approach which is widely used in the literature, this section finalises all the results, and provides comparison and analysis in terms of the main KPIs. The coverage KPI is not included in the analysis as the majority of designs obtained in the previous sections have been able to achieve the desired level of $95 \%$. The first KPI to be analysed is service level. The histogram illustrating the network service for all experiments is presented in Figure 7.31. The results are grouped by experiment which provide overall network service level in percentage for the stages $1,2 \& 3$ where possible. The white bars with the dashed lines indicate that results for particular stages were taken from the 'framework' experiment to perform the tests in the subsequent stages. The generated networks were selected from each experiment for comparison as follows:

1. Framework (Section 7.2)

(a) Stage 1 - s1-min

(b) Stage 2 - s2-5-min

(c) Stage 3 - s3-5-min

2. PB (Section 7.3)

(a) Stage 1 - taken from 'framework' experiment, s1-min

(b) Stage 2 - taken from 'framework' experiment, s2-5-min

(c) Stage $3-\mathrm{s} 3-\mathrm{pb}-5-\mathrm{min}$

3. WS2 (Section 7.4) 
(a) Stage 1 - taken from 'framework' experimentario, s1-min

(b) Stage 2 - s2-ws2-5

(c) Stage 3 - s3-ws2-5-min

4. I05 (Section 7.5)
(a) Stage 1-s1-i05
(b) Stage 2 - s2-i05-min

5. I6 (Section 7.5)

(a) Stage 1 - s1-i6

(b) Stage 2 - s2-i6-min

6. Standard (Section 7.6)

(a) Stage 1 - s1-3s-mean

(b) Stage $2-s 2-3 s-3$

(c) Stage $3-\mathrm{s} 3-3 \mathrm{~s}-3$

Starting with the 'framework' experiment (Figure 7.31) it can be seen that the service levels achieved in Stages 1 and 2 are almost identical, and higher than any other experiment could achieve, which demonstrates the capability of Stage 1 to produce realistic good quality network plans suitable for dimensioning purposes, as well serving as a good starting point for the subsequent optimization stages. Furthermore, this similarity in results illustrates that the Stage 2 optimization procedure performs in the most efficient way, thanks to the 'initial sectorization of cells' process (described in Section 6.1.2 where before optimization of the base station parameters begins this procedure identifies the optimal positioning of sector azimuths, which eventually significantly simplifies the following progress of the stage. The effectiveness of Stage 2 can also be outlined by the results produced in the 'WS2' experiment where the sectorization 


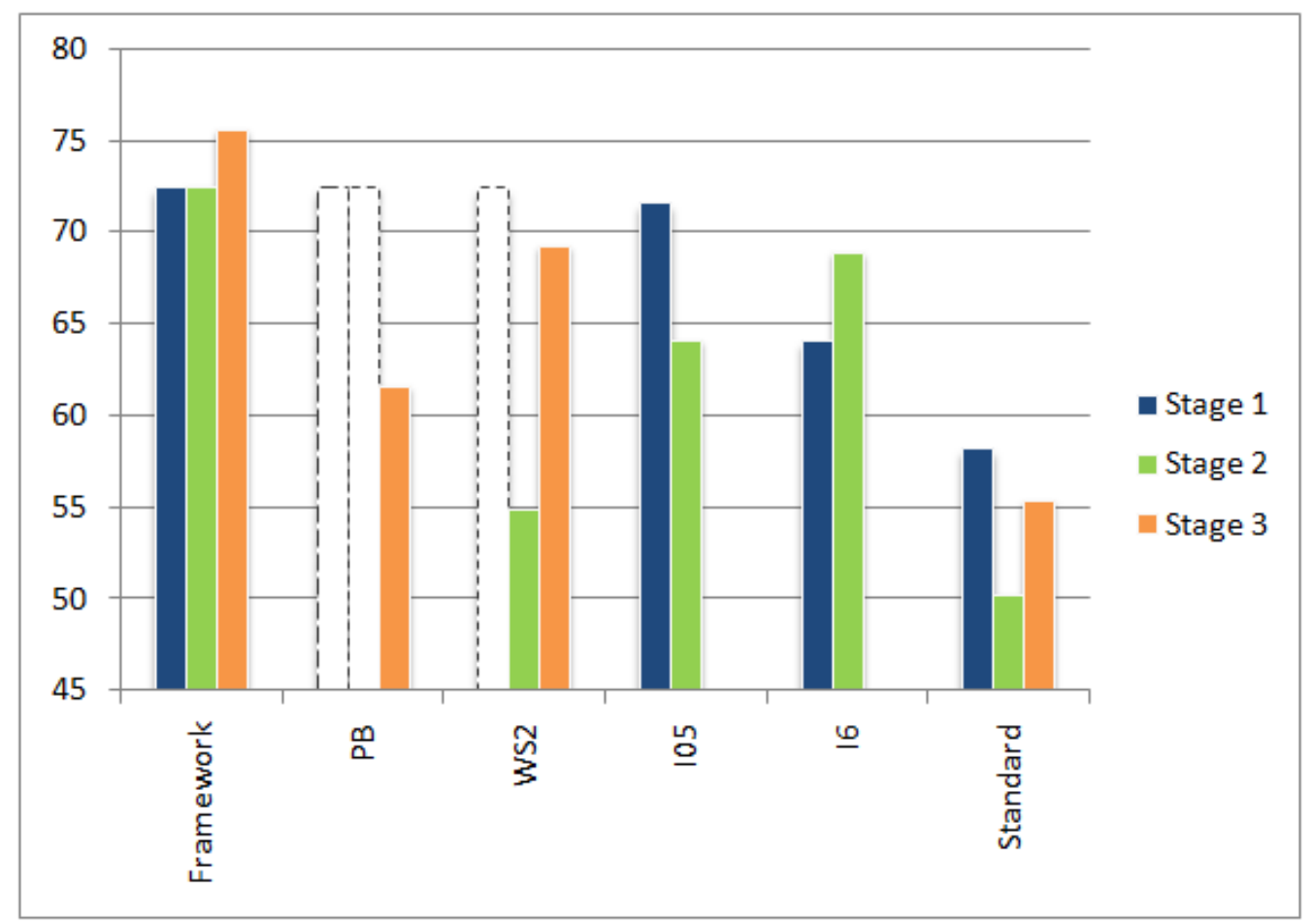

Figure 7.31: Comparison of the service levels achieved by different experiments (\%).

stage was simplified, which resulted in the reduction of the service level by $24.29 \%$ compared to Stage 2 from the 'framework' experiment. Stage 3 introduced further improvement to the service level reaching $75.66 \%$, which was an absolute maximum within the whole range of experiments and trials. As mentioned in Section 7.2 although the improvement between Stages 2 and 3 is not significant margin it shows that the first two stages produce very high quality solutions which leaves very little room for improvement in the last stage. This statement is also confirmed in the 'WS2' scenario where after the poor results produced in the second stage, Stage 3 achieves a significant improvement in the service by $26.2 \%$, which shows that it is capable of producing considerable modifications of the network plans where necessary. The 'PB' scenario where the base station sectors were allocated the same PermBase emphasizes the importance of the permutation base allocation procedure, which is ignored in the literature. The loss in the service level in comparison to the 'framework' scenario accounts 
for $18.57 \%$. The two experiments which involved tests on the capacity-interference parameter, namely 'I05' and 'I6', show that in both cases changing the parameter from the original value, (which were obtained by the series of experiments and concluded in Table 5.1), resulted in the misleading network plans produced in the first stage. This is confirmed in the Stage 2 results, where the slot assignment procedure together with the realistic model for the SINR calculation have corrected the impact of the inaccurate settings of Stage 1. The very low interference level in the Stage 1 network of 'I05' experiment results in the drop of service in the s-i05-min network by $10.59 \%$ and improvement in s2-i6-min by $7.39 \%$. This adds weight to the assumptions made in Stage 1 for the interference calculation and demonstrates the ability of the stage to be used as a dimensioning tool for WiMAX networks. Finally, the results of the 'standard' scenario demonstrate that simplification of the WiMAX network planning problem to that employed in the literature is impractical. The use of 3-sectored base stations and elimination of equipment parameters such as azimuth, tilt and antenna type from the decision variables of the optimization framework results in the worst results produced in all experiments. In comparison to the 'framework' experiment, the standard approach had a reduction in the service by $26.76 \%$ at the end of Stage 3. Moreover, this loss in service level is not the biggest drawback of the standard approach, which will be illustrated in the analysis of other important KPIs.

The next performance indicator to be analysed is profitability of the obtained networks, which are presented in the form of a histogram in Figure 7.32. The networks from the 'framework', 'PB' and 'WS2' experiments contain the same amount of equipment, including active sites and sectors. However the s3-5-min network of the 'framework' experiment shows the most favourable financial return on the investment because of the highest level of service. The 'WS2' experiment once again outlines the importance of the sectorization stage, where the network with the default configurations, not optimized for any defined objective produces results with minimum profit in the first financial year. However having been optimized in Stage 3 considerably improves profitability, but nevertheless does not match the performance of the 'framework' experiment by 


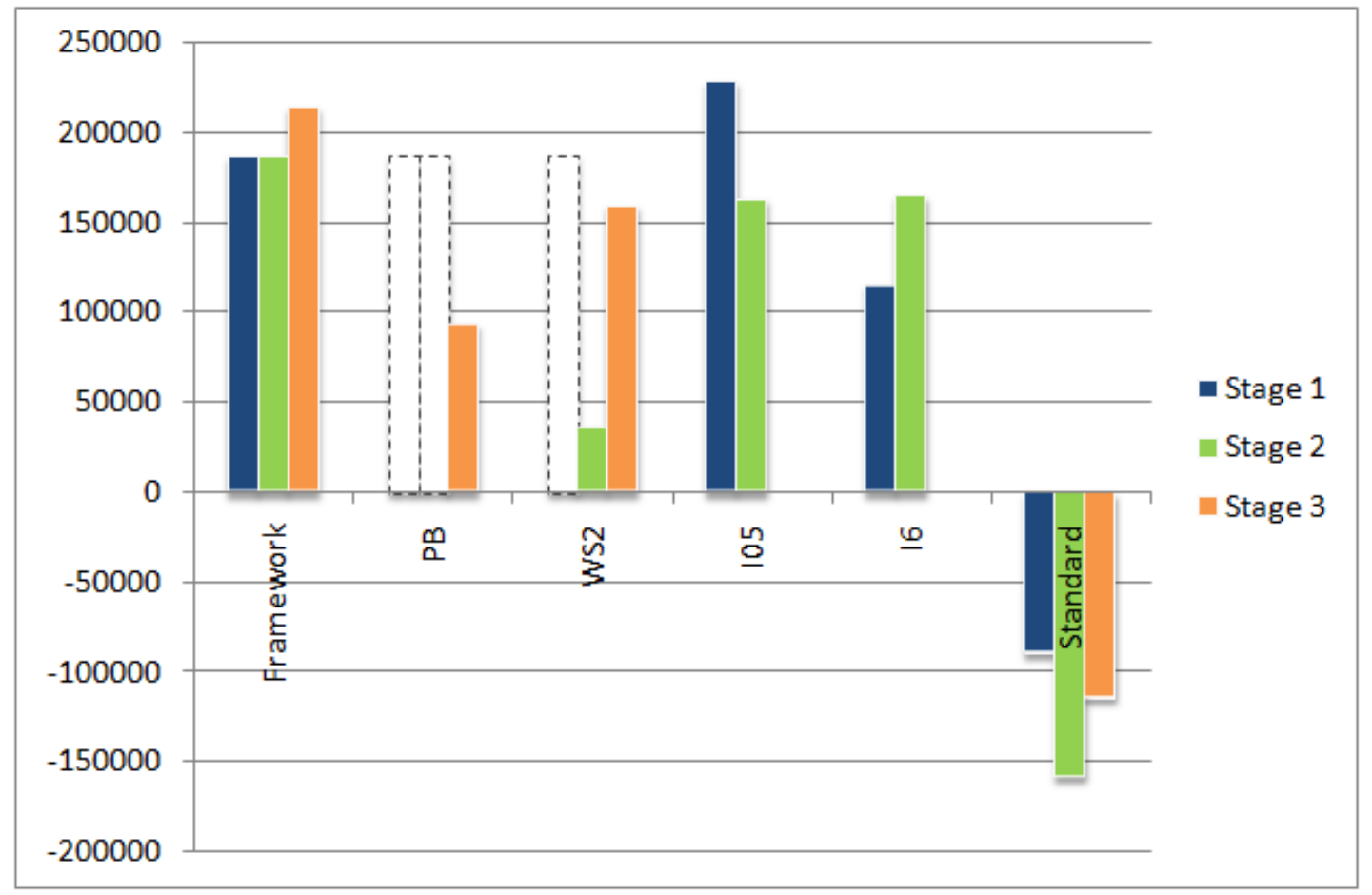

Figure 7.32: Comparison of the profit levels achieved by different experiments (€).

a considerable amount $(25.59 \%)$. The 'PB' scenario shows even greater reduction in profit by $56.16 \%$ in comparison to the 'framework' case, which indicates the significance of the PermBase allocation to the sectors, where even the simplified strategy proposed in this thesis can drastically improve the performance of all KPIs. The 'I05' scenario as was mentioned in the service level KPI analysis proves to be misleading. Stage 1 when used as a dimensioning mechanism for WiMAX networks with incorrect (lowered) settings of the capacity-interference parameter may show overestimated profitability of a potential network, which in turn may result in the development of an misleading financial strategy by a service provider for network deployment. This is confirmed by the sectorization stage which reduced the profit of the network by 28.44\%. The converse can be observed in the 'I6' scenario where the parameter was increased, which resulted in the negative impact on the first stage network plan, which is then improved by $42.54 \%$ after sectorization. Finally, the 'standard' experiment con- 
firms in financial terms as well, that this approach is unsuitable for planning purposes. The low service level produced and the high expense caused by the activation of the increased number of sites for BS deployment in comparison to the 'framework' scenario (€585000 and $€ 432000$ respectively) results in the significant losses.

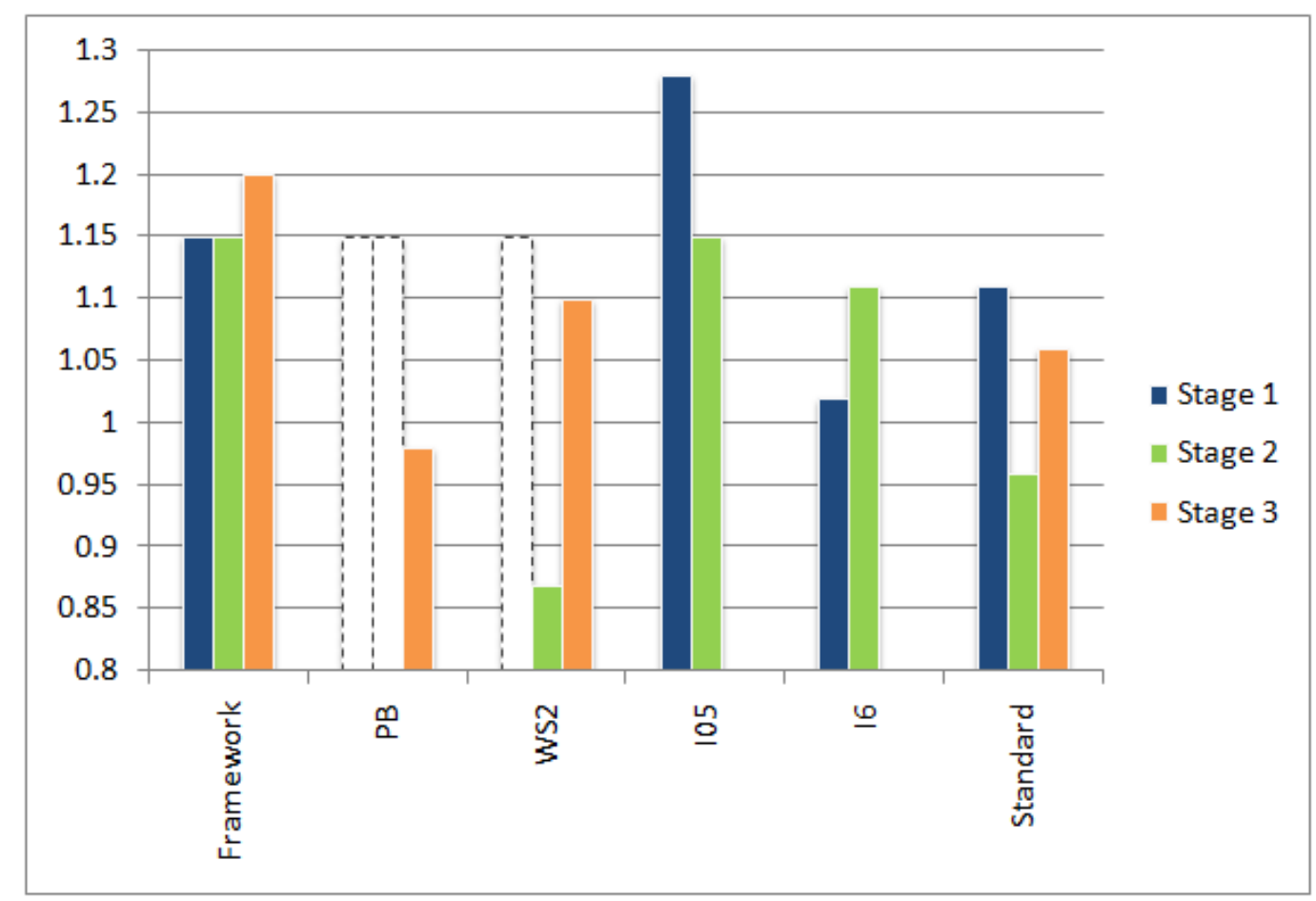

Figure 7.33: Comparison of the spectral efficiency levels achieved by different experiments $(\mathrm{bps} / \mathrm{Hz})$.

An important technical criterion of a network is how efficiently the overall system uses the available spectrum. The comparison of spectral efficiency for different experiments is displayed in Figure 7.33. From the 'framework' experiment it can be seen that the results are fairly consistent, where Stage 1 and 2 networks have the same spectral efficiency of $1.15 \mathrm{bps} / \mathrm{Hz}$ and an improved value of $1.2 \mathrm{bps} / \mathrm{Hz}$ in the third stage. The 'PB' and 'WS2' experiments show similar losses in the efficiency relative to other KPIs described above, with a degradation by $18.33 \%$ and $8.33 \%$ respectively. The most notable result however was shown by the 'I05' scenario, where the lower parameter of the SINR expression in Stage 1 results in the spectral efficiency peaking at the level of 
$1.28 \mathrm{bps} / \mathrm{Hz}$ which was revised after the second stage to $1.15 \%$. In spite of the poor performance of the standard approach in the service and profitability indicators, the spectral efficiency achieved a reasonable level after Stage 3 resulting in 1.06 bps/Hz. This demonstrates that all stages of the optimization framework strive to optimize the network to use the spectrum as efficiently as possible.

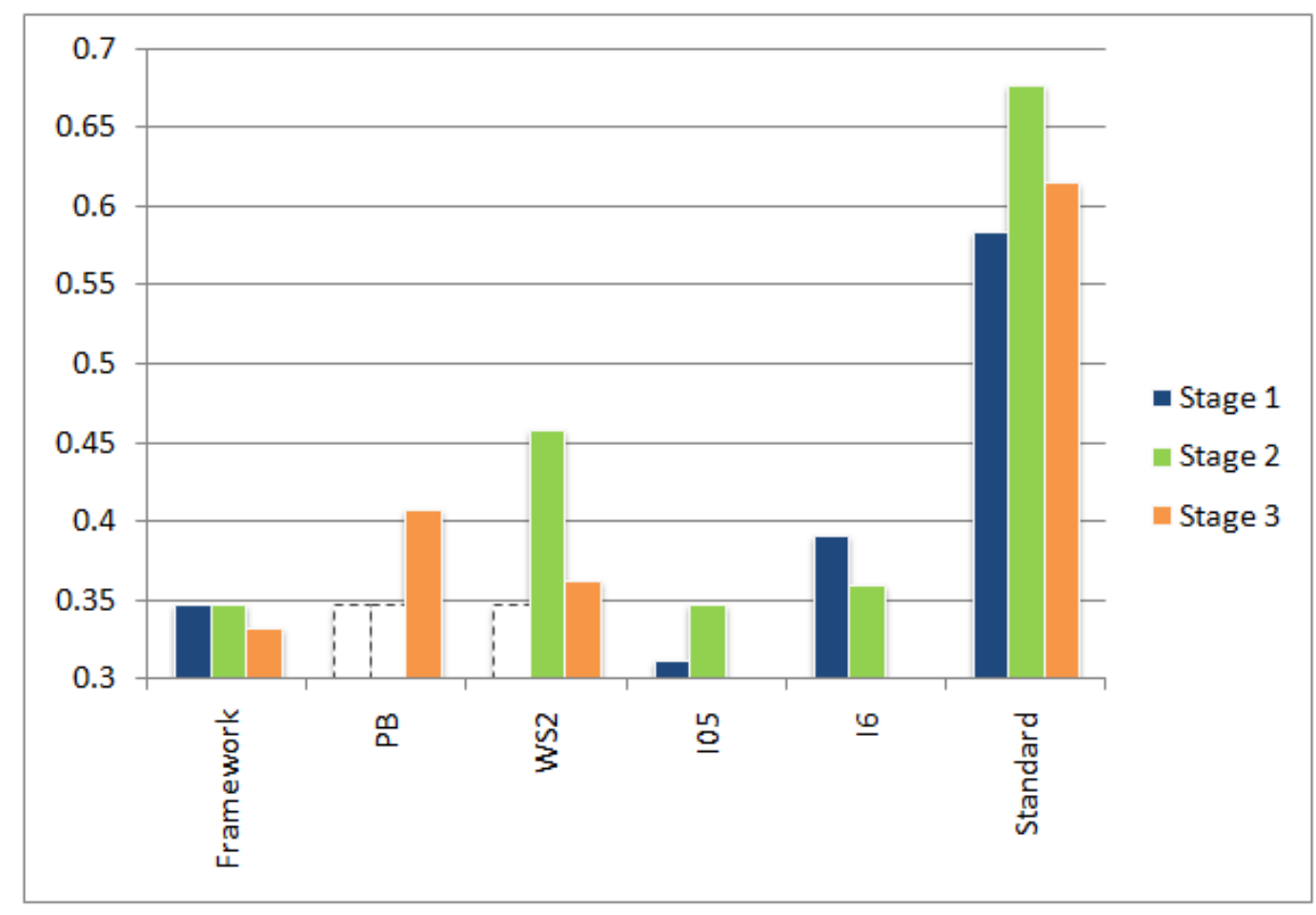

Figure 7.34: Comparison of the cost of traffic levels achieved by different experi$\operatorname{ments}(€ / \mathrm{Kbps})$.

The last KPI to be analysed is the cost of traffic, presented in Figure 7.34. The 'framework' experiment networks, as always, show that Stage 3 produces the most cost effective plan. Experiment 'PB' shows the poor cost efficiency of the network, degrading the result of the 'framework' scenario by $22.89 \%$, as well as in the 'WS2' case with a $9.34 \%$ reduction. The result of the 'standard' scenario, with a significant $85.24 \%$ deterioration of the cost-efficiency of the network. This however again shows that this approach is far from being suitable for planning practical networks for WiMAX technology. 
Chapter 8

\section{Simulation}

This chapter considers the validity and viability of WiMAX networks generated using the proposed optimization framework. These networks are evaluated by the use of OPNET wireless network simulator [3]. The OPNET Modeler Wireless Suite is designed to provide high fidelity modelling, simulation, and analysis of a broad range of wireless networks. Along with network evaluation of performance it also allows consideration of proprietary wireless protocols, such as access control and scheduling algorithms. The modeler Wireless Suite supports any network with mobile devices, including cellular (GSM, CDMA, UMTS, IEEE 802.16 WiMAX, LTE, etc.), mobile ad hoc, wireless LAN (IEEE 802.11), personal area networks (Bluetooth, ZigBee, etc.) and satellite. The version of the simulator used in this thesis is OPNET Wireless Suite version 15.0.

The key features of OPNET:

- fast simulation engine among leading industry solutions

- hundreds of wired/wireless protocol and vendor device models with source code

- object-oriented modelling

- hierarchical modelling environment

- scalable wireless simulations incorporating terrain, mobility, and multiple pathloss models 
- customizable wireless modelling

The OPNET WiMAX Specialized Model which is available for the OPNET Modeler Wireless Suite supports the IEEE 802.16-2004 and IEEE 802.16e-2005 standards. The main specifications and configurations of the model used are described in the following section.

\subsection{OPNET Wimax model}

OPNET's WiMAX model was developed with guidance from over fifty industry leaders including Alcatel-Lucent, France Telecom, Fujitsu, KDDI, NEC, Samsung, and Sony Ericsson. OPNET offers the most feature-rich and comprehensive WiMAX network simulation capability available today, including support for scheduling mechanisms, adaptive modulation and coding, mobility and handoffs, power management, interference models, and fading models. Some of the key features are:

\section{PHY}

- TDD

- OFDMA, SOFDMA

- Co-channel interference (PermBase, subcarrier overlap computation)

- MIMO

- Multi-path fading

- Path-loss modelling (ITU, Erceg) 
MAC

- Service flow configuration and mapping traffic to service flows

- Bandwidth request and grant mechanism

- BS scheduler for UL and DL connections

- Scheduling services for UGS, ertPS, rtPS, nrtPS, BE

- Adaptive modulation and coding

- ARQ, HARQ

- Ranging (initial and periodic)

Framing - DL/UL MAP generation, burst rectangulation

Network features - multiple cell networks, multi-sector base stations, IP connectivity The main building components used in OPNET for WiMAX simulation are SS nodes (or subscriber station), BS nodes, WiMAX Config node, wireless and wired links, and nodes for applications and profiles. SS and BS nodes have attributes that allow specification of the PHY configurations of each node, whereas WiMAX Config node specifies the settings of the whole WiMAX system. These attributes can be seen in Figures $8.1,8.3$.

The default palette of node models included in OPNET's WiMAX library is fairly limited, and is insufficient to simulate the network plans obtained from the framework. However with the extensive selection of editing tools provided in OPENT the following objects and models were modified to complement the library:

\section{BS node model}

By default OPNET supports only two types of base station models, namely one and three-sectored. Therefore in order to perform the simulation of the networks with the 


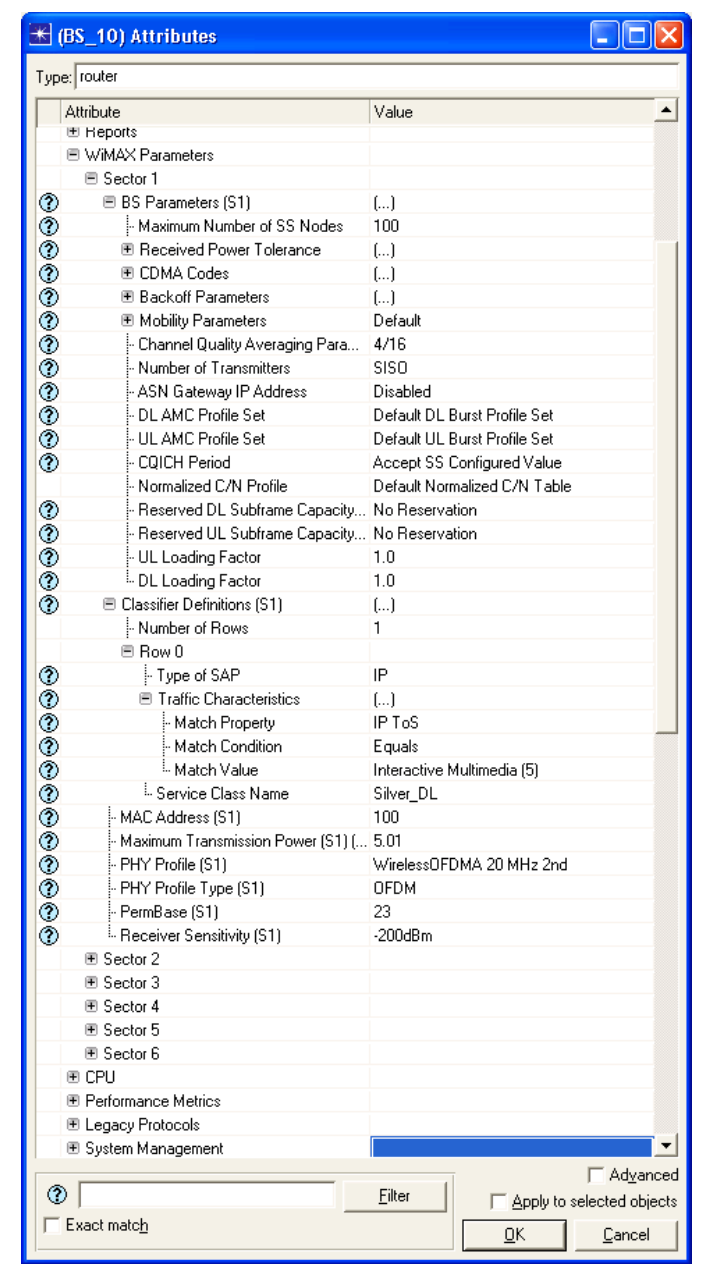

Figure 8.1: WiMAX BS node attributes

same equipment specifications as used in optimization five new BS models were created using the OPNET Node editor, varying the sectorization between 2 and 6 sectors. Each sector can be configured individually with a standard set of PHY parameters. In comparison to the optimization framework where the direction of an antenna radiation pattern is defined by the azimuth and tilt of each sector, in OPNET it is specified by the antenna targeting point with coordinates $\mathrm{x}, \mathrm{y}, \mathrm{z}$ and the position of the antenna within the network subnet. The set of the base station attributes defined for each sector includes BS position, MAC address (which allows to associate users/subscribers to a particular BS sector), maximum transmission power (in Watts), PermBase, antenna pattern model and the targeting point of antenna. The rest of the settings which are 


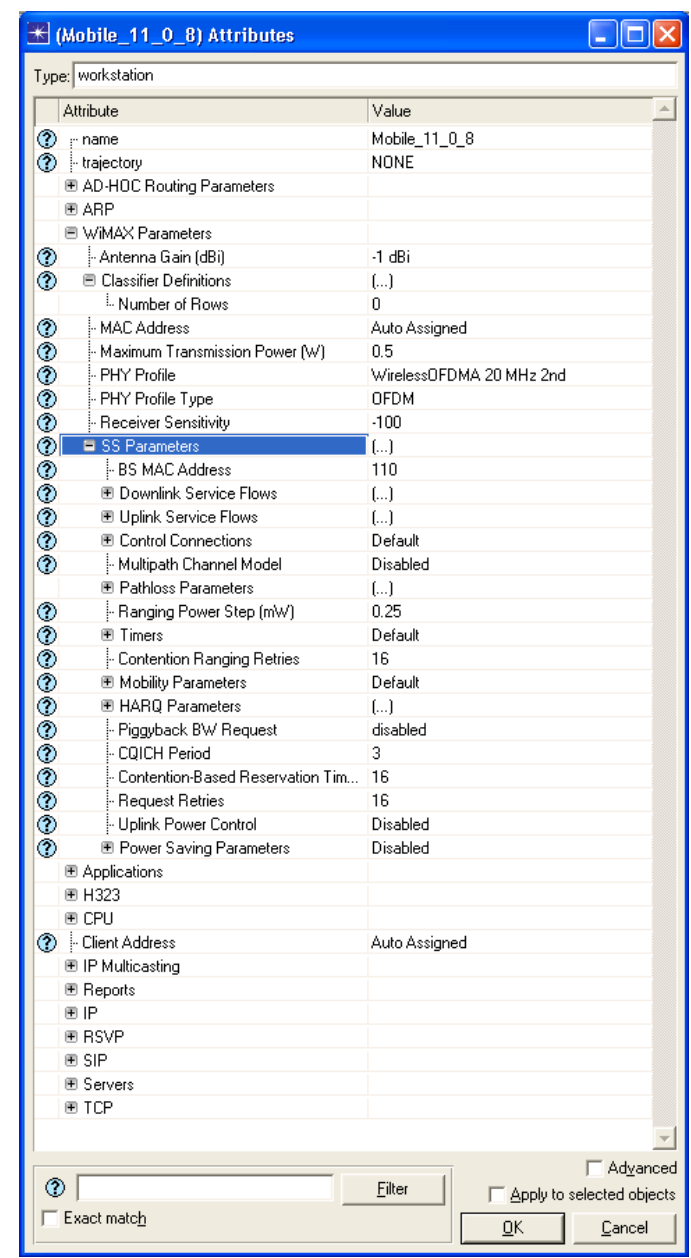

\section{Figure 8.2: WiMAX SS node attributes}

shown in Figure 8.1 are set to the same values for all the BS sectors in the network.

\section{Antenna patterns}

The OPNET Modeler provides only two types of antenna pattern in the palette for WiMAX network simulation, these are omni-directional and sectored with 120 degrees radiation pattern. The four types of antennas used in the optimization framework were recreated with the use of the Antenna pattern editor provided by OPNET and an example of the 90 degrees pattern created can be seen in Figure 8.4

\section{Propagation model}

The propagation (or pathloss) model in OPNET is specified individually at each sub- 


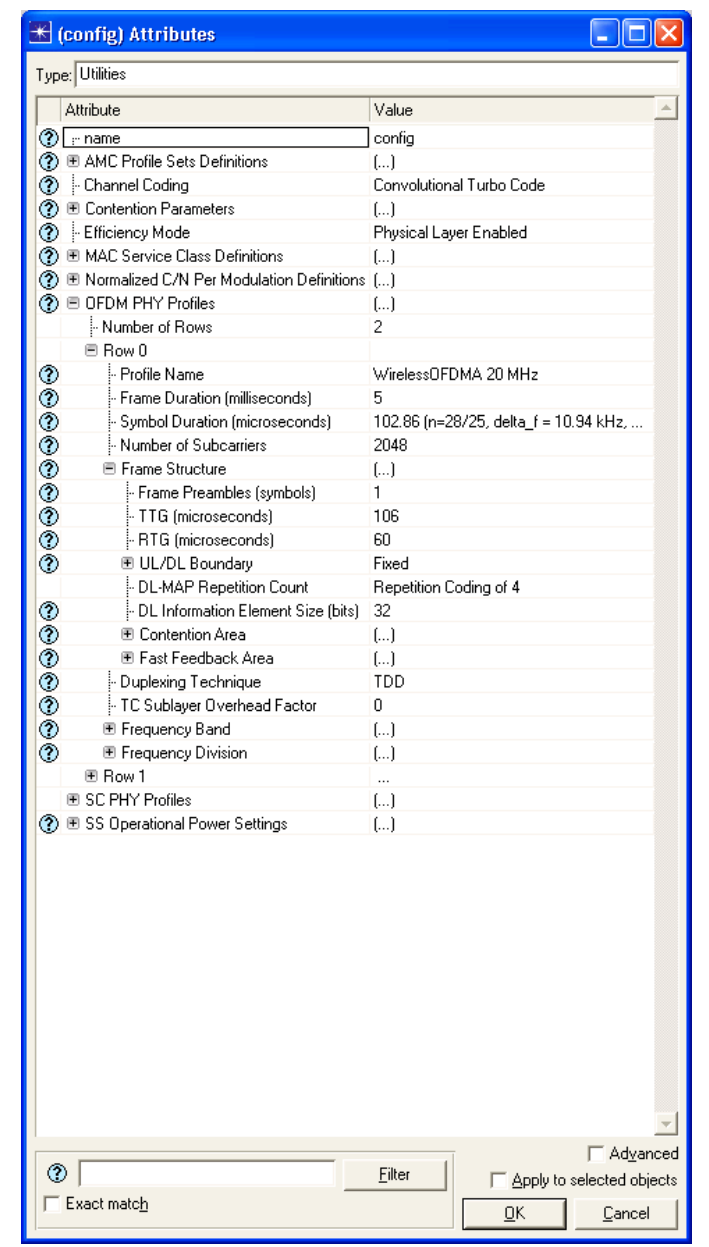

Figure 8.3: WiMAX Config node attributes

scriber node. The default options of the models provided are:

- Free Space

- Suburban Fixed (Erceg)

- Outdoor to Indoor and Pedestrian Environment

- Vehicular Environment

Each pathloss model is appropriate for a certain kind of environment through which the signal propagates before reaching the receiver. The "Free Space" pathloss model refers to the classical free space pathloss. The "Suburban Fixed (Erceg)" pathloss model is 

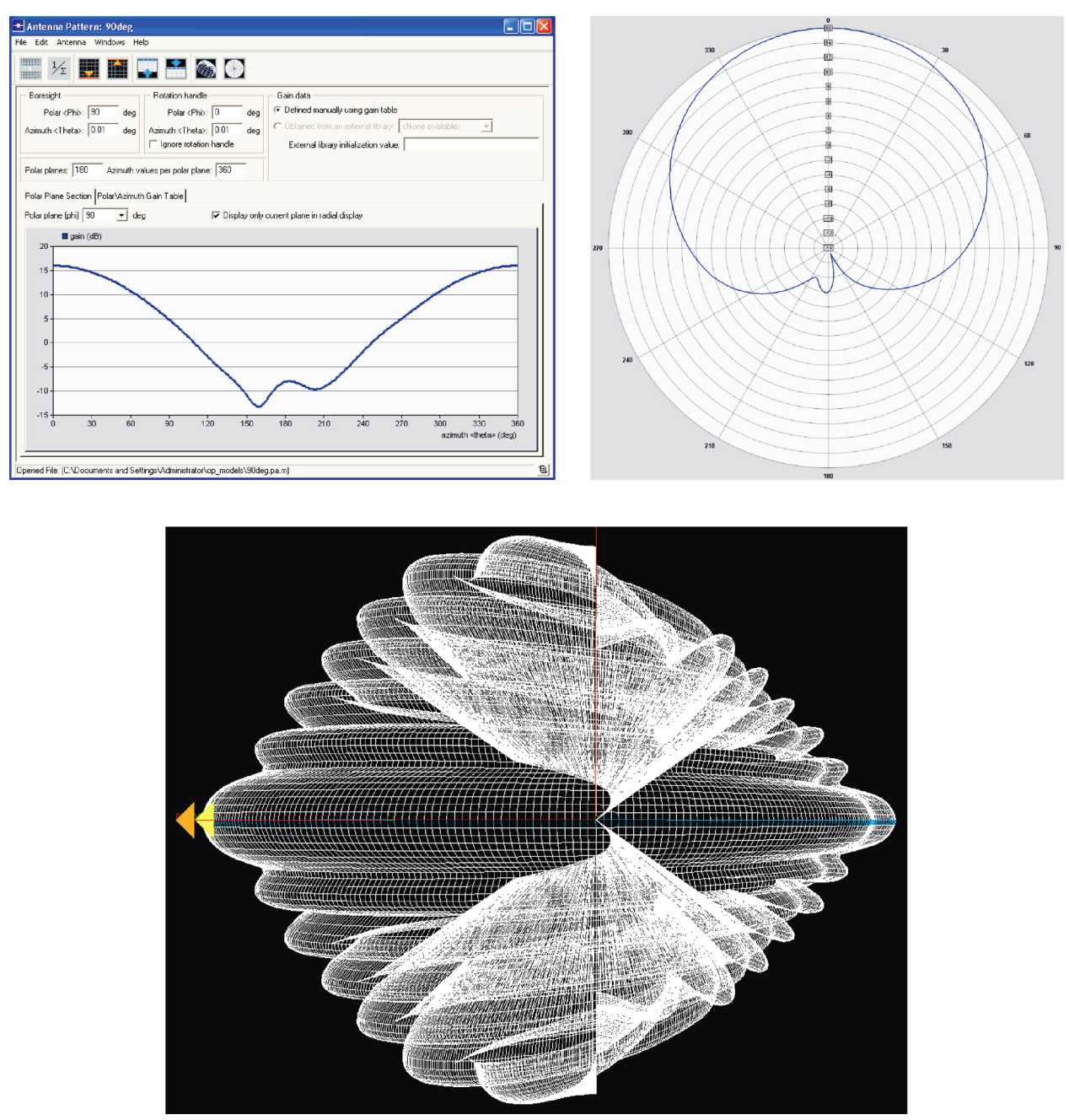

Figure 8.4: OPNET antenna editor - 90 degrees pattern example

defined in [55]. Erceg's model is also referenced in the IEEE802.16a-03/01 standard [56]. The "Outdoor to Indoor and Pedestrian Environment" and the "Vehicular Environment" are pathloss models described in the "Radio Tx Technologies for IMT2000" white paper of the ITU [120].

However, as we employ our own propagation model in the optimization framework which provides pathloss figures in the form of an array from all the site locations to all the STPs, this was recreated in OPNET. The wireless communication between nodes in OPNET is implemented through Transceiver Pipelines, where each pipeline denotes a sequence of specific calculations. Six pipeline stages are associated with radio trans- 
mitter: receiver group, transmission delay, link closure, channel match, Tx antenna gain and propagation delay. Eight stages are associated with the radio receiver: Rx antenna gain, received power, interference noise, background noise, Signal-to-Noise Ratio, Bit Error Rate, error allocation and error correction. Therefore, in order to accommodate the pathloss model used in the network optimization framework the 'received power' pipeline stage was modified to read the pathloss figures from a file containing all the propagation attenuations and then match the pairs of base station site locations and users to obtain the required pathloss value.

\subsection{Simulation approach}

The OPNET Modeler supports the import of network topologies from different sources, including XML files. As the use of the network instances considered in this thesis is quite considerable with thousands of users it is impractical to create topologies manually. Therefore, an application was created to convert the results from the optimization framework to the XML files with an OPNET structure. The XML files include all the information about the network nodes and the specifications of the node attributes which differ from the default settings. An example of the imported network topology can be seen in Figure 8.5 .

One of the difficulties encountered with the OPNET simulator, in particular related to the WiMAX standard model, is its inability to perform simulation of the network plans consisting of thousands of users. To overcome this problem the users within each base stations sector were clustered and combined in order to reduce the size of the network. This procedure is as follows:

1. all the serviced users within each sector are sorted in ascending order of the distance to the serving BS

2. beginning with the first user in the list, 10 are selected in order 


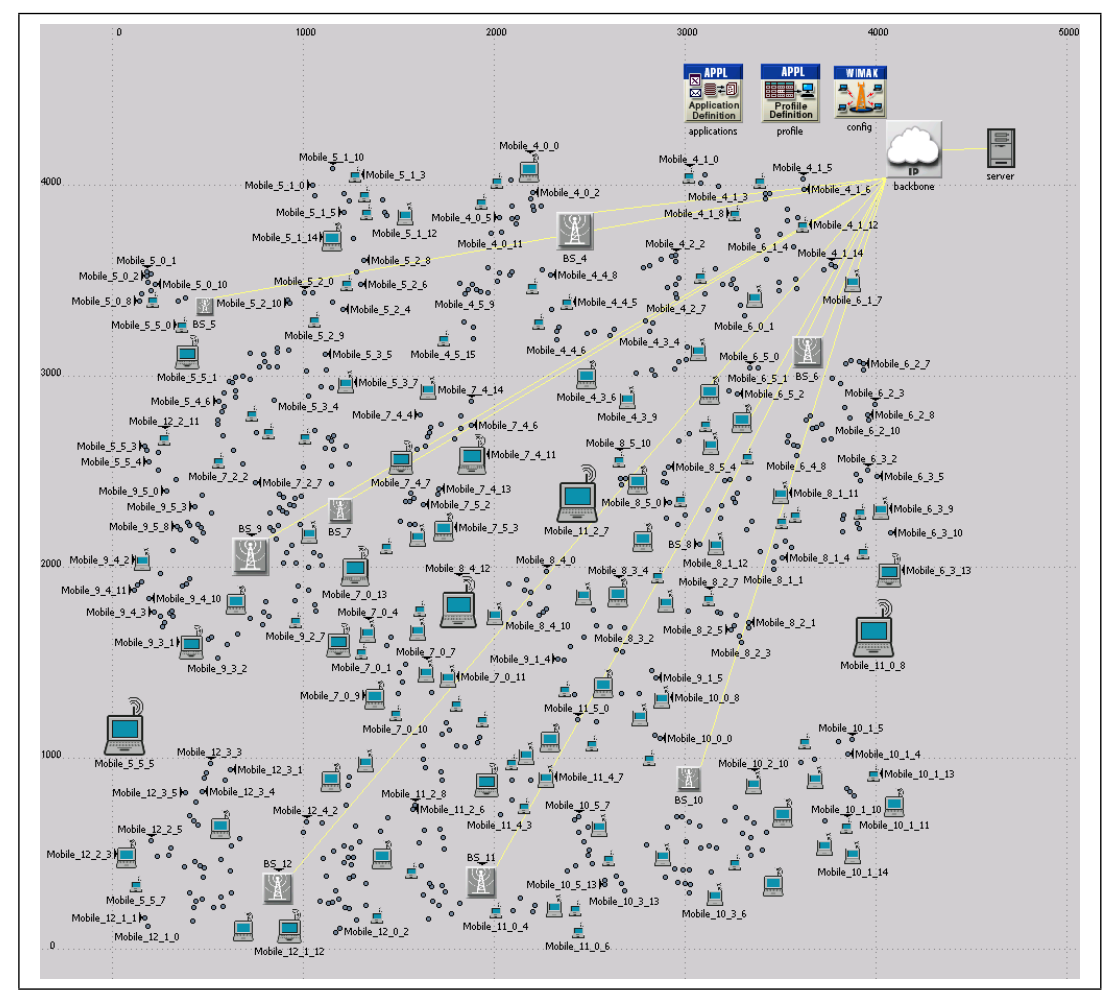

Figure 8.5: WiMAX network topology example in OPNET

3. their centre of mass is calculated

4. the position of the user located closest to the centre of mass is determined

5. all the selected users are then replaced with one and positioned in the location which has been determined in the previous step

6. the traffic demand of the final user is an aggregate of all the selected users.

Although a network plan with clustered and grouped users differs from the original version, this procedure overcomes the limitations of OPNET and makes it possible to perform simulation of the overall network.

Traffic in OPNET can be defined in different ways and one of them which was employed in our example is the use of applications. Applications can be created and modified in the 'Application Config' node where a range of application types is specified including database, email, FTP, HTTP, print, remote login, video conferencing 
and voice. Every application offers different traffic load options. For example, "Web Browsing (Heavy HTTP 1.1)" indicates a web application performing heavy browsing using HTTP 1.1. For simulation purposes we have selected the 'video conferencing' application which can be set using the following attributes:

- frame interarrival time information

- frame size information (bytes)

- symbolic destination name

- type of service

- RSVP parameters

- traffic mix

As previously described the sector users are grouped in clusters of ten for the simulator to cope with the size of the network. This means that most of the users after the clustering procedure will have a traffic demand equivalent to the demand of 10 users, or $2 \mathrm{Mbps}$ in the DL. However, the number of serviced users in the base station sectors rarely equal 10, which results in the variation of the traffic demand for the last user group (from 1 to 9 users). In other words, if the total amount of serviced users in a sector accounts for 108 users, this means that clustering procedure will produce ten groups of 10 users which leaves the last group with only 8 users, and a traffic demand of 1.6 Mbps. Therefore, 10 different 'video conferencing' applications were created by varying the attributes provided above in order to support 10 levels of traffic demand, from 0.2 Mbps to 2.0 Mbps. The specified applications are then used while creating user profiles on the "Profile Config" object. A profile describes user activity over a period of time and may consist of many different applications. For example, a "Human Resources" user profile may contain "Email", "Web" and "Database". Various loading characteristics can be specified for the different applications on this profile. The 
profiles created in this object will be referenced by the individual subscriber nodes to generate traffic. As we have 10 'video conferencing' applications the same number of profiles were created. The starting time of each profile was specified by a random uniform distribution with a minimum outcome of 50 seconds and maximum of 100 seconds, which means that the transmission of traffic for different users will start between 50 and 100 seconds after the simulation begins. The 50 second minimum is used so that network can be initialized.

As mentioned previously each user node can be configured individually. However, the main settings to modify are the MAC address of the associated BS, transmission power, the pathloss model, modulation and coding specified in the service flow settings, and the application profile used. OPNET provides a great deal of flexibility in other settings which can be seen in Figure 8.2, however in our case they are set to the default values.

Once all the BS and SS nodes are created and configured, as well as the applications and profiles, it remains to connect these base stations to the network backbone and the server where all the traffic applications are originating. The links used to connect BSs to the backbone are SONET OC-12 (Synchronous Optical Networking, Optical Carrier with multiplier of 12) with transmission speeds of up to $622.08 \mathrm{Mbps}$, and between the backbone and the server SONET OC-48 a speed of up to $2488.32 \mathrm{Mbps}$ is available.

\subsection{Simulation results \& performance analysis}

In order to evaluate the performance of the proposed WiMAX network optimization framework, 10 network plans were selected from the whole range of the results described in Chapter 7. First, two network plans were selected with the worst service level of $58.86 \%$ and the best of $75.66 \%$. Then, eight remaining plans were determined with the service level between the worst and the best network plans in steps of $1.8666 \%$. Table 8.1 provides the list of selected network plans (from the experiments 'WS2', Stages 2 and 3 of 'framework'), where the 'service step' column giving the 
service level equally split between the best and worst values, and the following two columns giving the service level in percentage and Mbps terms for the network plans closest to the values of 'service step'.

\begin{tabular}{lcccc}
\hline ID & Network plan & Service step (\%) & $\begin{array}{c}\text { Network service } \\
(\%)\end{array}$ & $\begin{array}{c}\text { Network service } \\
(\text { Mbps })\end{array}$ \\
\hline 1 & s3-ws2-1-5 & 58.86 & 58.86 & 1010.4 \\
2 & s3-ws2-4-3 & 60.73 & 60.92 & 1045.8 \\
3 & s3-ws2-2-5 & 62.59 & 62.57 & 1074.0 \\
4 & s3-ws2-2-2 & 64.46 & 64.42 & 1105.8 \\
5 & s2-1-4 & 66.33 & 66.33 & 1138.5 \\
6 & s3-ws2-4-1 & 68.19 & 68.2 & 1170.8 \\
7 & s2-3-2 & 70.06 & 70.13 & 1203.8 \\
8 & s2-5-1 & 71.93 & 71.77 & 1231.9 \\
9 & s3-1-1 & 73.79 & 73.85 & 1267.7 \\
10 & s3-5-2 & 75.66 & 75.66 & 1298.7 \\
\hline
\end{tabular}

Table 8.1: Network plans selected for simulation in OPNET

The duration of the simulation was set to 150 seconds which proved to be sufficient for the network's traffic to settle. The real execution time of the simulation runs was on average 4 hours. Figure 8.6 illustrates the total throughput variation over the simulation period for the network plan 1. It can be seen that it steadily increases starting from 50 seconds of the simulation time when the users gradually begin activating their traffic profiles. Towards the end of the simulation time throughput converges to the value of 875.4 Mbps. The breakdown of the simulation results and comparison to the results of the optimization framework for 10 selected network plans is presented in Table 8.2. On average, the service level of the simulation results is lower by $15.67 \%$ in comparison to the optimization framework. The main reason behind the reduction of the system throughput level is the approximation used to simulate the network plans. In other words the procedure of clustering and grouping users performed in the pre-simulation 


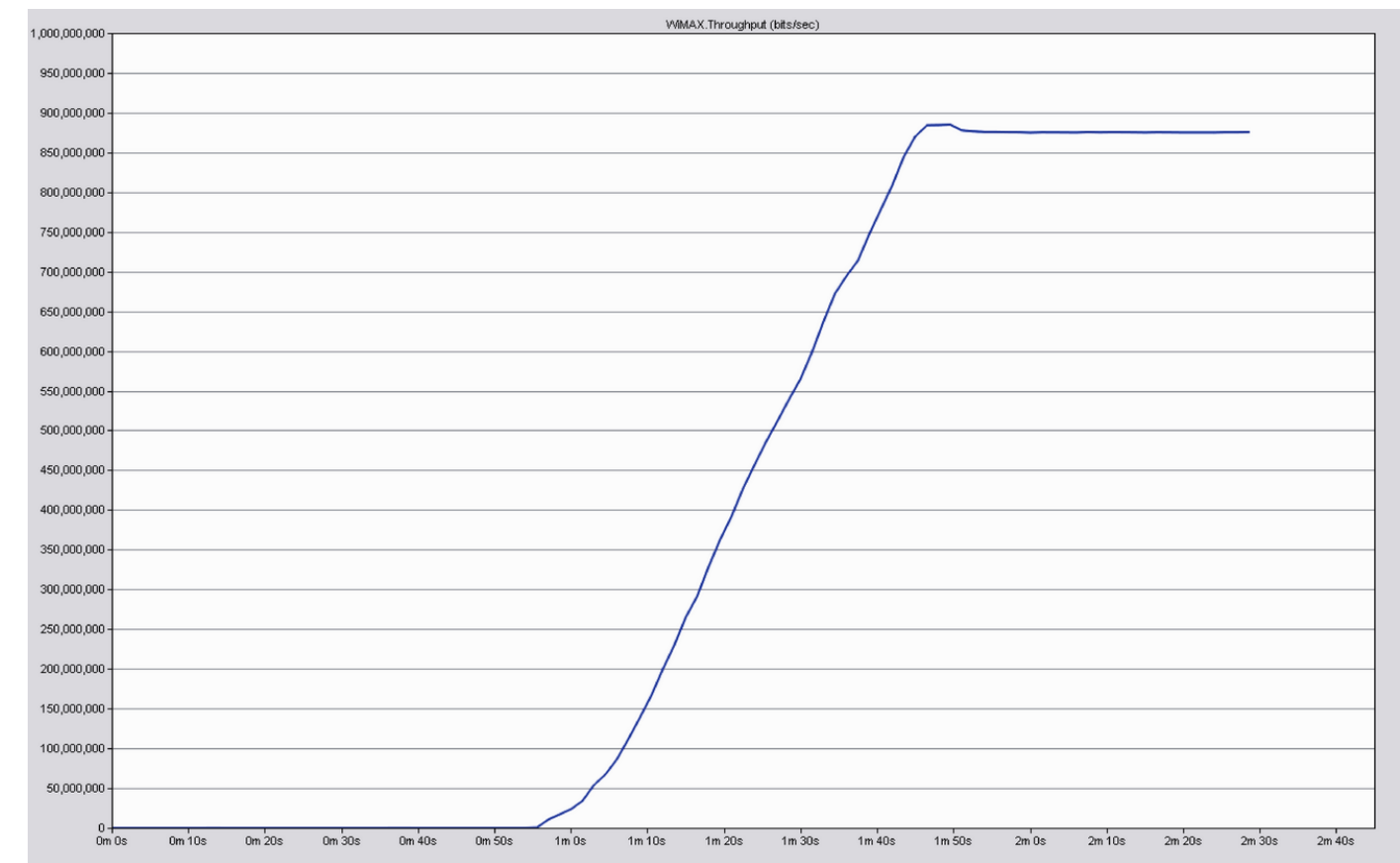

Figure 8.6: Total network throughput of the network plan 1 in OPNET simulation.

\begin{tabular}{lcc}
\hline Network ID & Optimization service (Mbps) & OPNET service (Mbps) \\
\hline 1 & 1010.4 & 875.4 \\
2 & 1045.8 & 951.6 \\
3 & 1074.0 & 932.2 \\
4 & 1105.8 & 932.4 \\
5 & 1138.5 & 970.2 \\
6 & 1170.8 & 1027.3 \\
7 & 1203.8 & 928.0 \\
8 & 1231.9 & 1020.7 \\
9 & 1267.7 & 938.4 \\
10 & 1298.7 & 1138.4 \\
\hline
\end{tabular}

Table 8.2: Comparison of the results in terms of service between optimization framework and OPNET simulation. 
stage to reduce the size of networks and to make it feasible for the OPNET Modeler to simulate these network designs. This results in the subsequent approximation of the pathloss figures for the grouped users which inevitably leads to the alteration of the received SINR and the use of different modulation and coding schemes. The overall comparison of the service level achieved by the 10 selected network plans in the optimization framework and the OPNET simulations is illustrated in Figure 8.7. Although the total throughput figures of the simulated network plans are lower in comparison to the results of the optimized networks, both show an upward trend. In particular it can be seen that both the red line of the optimization framework and the black line of the simulation results (fitted curve) both show an upward trend and helps validate the quality of the WiMAX network plans produced by the optimization framework.

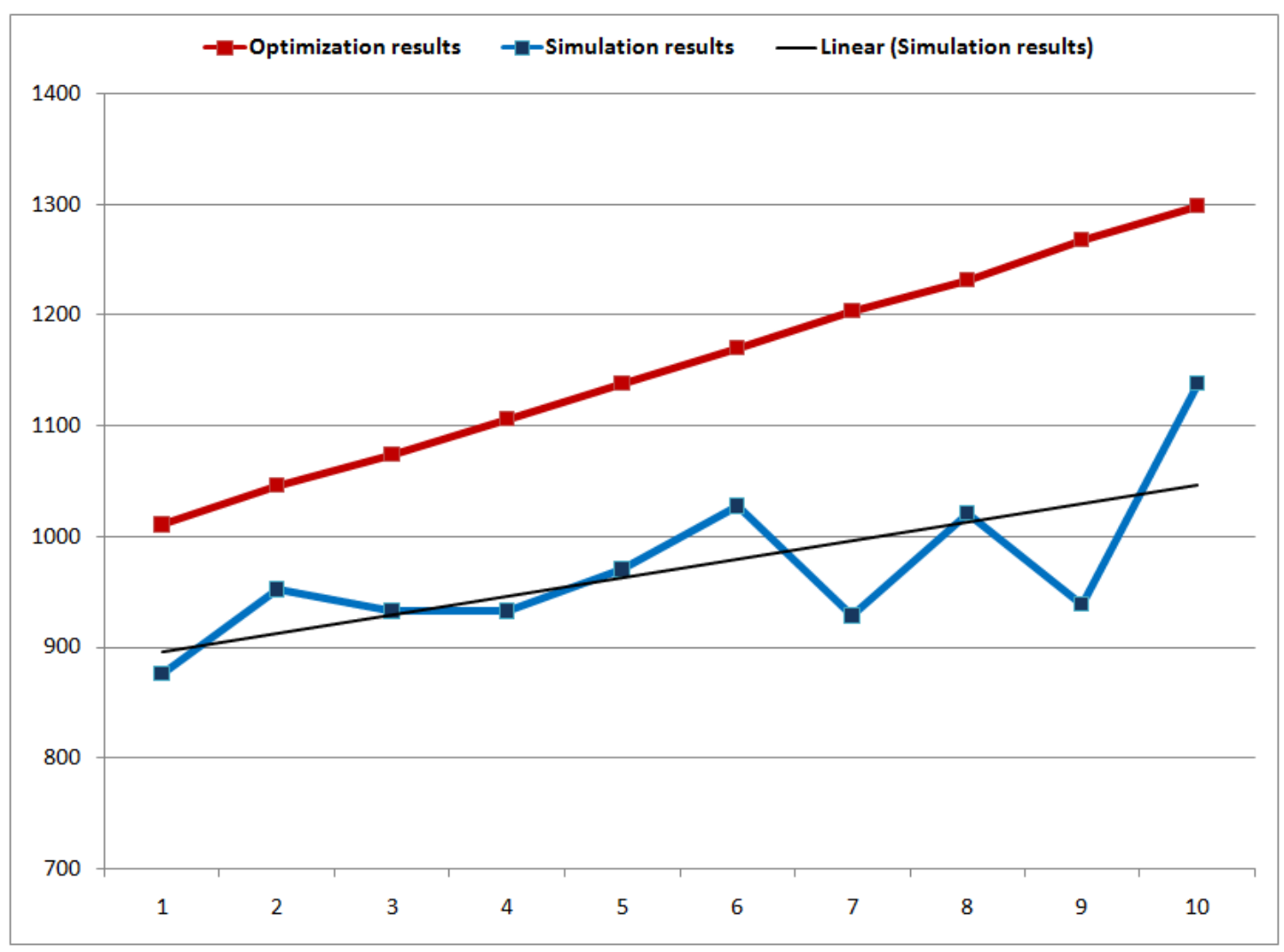

Figure 8.7: Graphical comparison of the results between optimization framework and OPNET simulation for 10 selected network plans in terms of the service level in Mbps. 


\section{Chapter 9}

\section{Conclusions and future work}

The main requirements of the network planning problem imposed by service operators is to design and deploy a cost-effective network which will increase the financial return on the investment. In other words to increase profit for the company while improving the quality of service they provide to network users, enhancing the coverage level and the overall capacity of the system in order to reduce coverage holes within the network topology. Historically, the cell planning problem was solved by manual techniques. However, this approach has been shown to be impractical for network engineers once the technologies providing wireless access have become more accessible, thus increasing the number of subscribers and services worldwide, and leading to the increase in complexity of network planning. Thus more emphasis has since been made on the development of automated cell planning techniques in order to design more efficient and, most importantly, feasible network solutions.

The literature review in Chapter 2 presented the progress and evolution in the field of automated planning for a variety of wireless access technologies including the development of models and optimization techniques for frequency and cell planning. It showed that for mature technologies such as second and third generation systems there has been a lot of research devoted to developing optimization techniques covering all possible challenges imposed by the technologies. However, for the relatively new Mobile WiMAX standard there is a limited number of studies aimed at the network planning problem which are either simplified in terms of consideration of all the WiMAX-specific features, including subchannelization, slot allocation, PermBase as- 
signment, specifics of SINR calculation and system throughput estimation, or consider simplified planning strategies where base station configurations are limited to $3 \mathrm{sec}-$ tors and eliminating consideration of azimuth, tilt and antenna pattern type, as well as considering small network instances which do not represent the realistic complexities of the network planning. These omissions were addressed in the optimization framework of this thesis. Chapter 3 presents the system model and crucial issues such as the propagation model used for planning WiMAX networks in the $3.5 \mathrm{GHz}$ frequency band, coverage and service requirements, resource allocation problems including subchannelization with consideration of the permutation scheme, slot partitioning and allocation, interference calculation and realistic throughput estimation. Chapter 4 describes the optimization framework in general presenting such issues as the optimization algorithm, its configurations and settings, objectives which were used to evaluate the quality of the network plans during the optimization process, as well as the key performance indicators used to evaluate the system performance of the final solutions.

Another problem addressed in this thesis which traditionally gets little attention in the literature is network dimensioning, which is even less studied for WiMAX technology. Chapter 5 presents a novel approach to the dimensioning problem which in comparison to traditional techniques based on the analytical evaluation of coverage and capacity requirements, builds on the optimization algorithm and not only identifies the amount of required equipment, or the number of sites to be activated, but also provides the exact selection of site locations required for provision of a good level of coverage and service, as well as identifying the set of RTPs, to be serviced by each selected site. Moreover, it gives a breakdown of the required number of sectors for the deployment of base stations at each selected site and therefore allows more accurate estimations on future profit. Another benefit of this approach is in the use of the dimensioning stage as a part of the whole optimization framework, or in other words as a precursor to the subsequent optimization stages for sectorization and configuration of network plans.

Chapter 6 presents the subsequent stages of the optimization framework, where Stage 
2 performed the sectorization of the base stations with omni-directional antennas and aims at achieving similar network results as in the first stage, and is accomplished by the introduction of an additional cost function objective. It also includes the process of initial sectorization which further reduces the complexity of the problem and allows us to find better configurations more efficiently. Stage 3 included the full range of neighbourhood moves to perform the final configurations of the base stations in order to gain the best possible result in terms of the defined KPIs.

The optimization framework for WiMAX network planning introduced in this thesis, including the dimensioning stage acting independently, was tested in a variety of ways in order to show the effectiveness of the proposed approach, the results being presented in Chapter7. First, a series of experiments (Section 7.2) was conducted to evaluate the network optimization framework. The outcome confirmed that Stages 1 and 2 produced very similar results despite the fact that Stage 1 generated artificial network plans, as well as it including a number of assumptions to overcome the problems of SINR calculation and capacity estimation. Stage 3 further improved the quality of network plans and within the overall range of testing scenarios produced the best results. Another conclusion made is that for the size of the experimental network scenario considered in this thesis a $20 \mathrm{MHz}$ channel is not sufficient to provide full area service. The next experiment (7.3) was aimed at evaluating the effect of the PermBase assignment and estimating the deterioration in service level in comparison to the case when PermBase is fixed. The results show that on average the loss in service is approximately $20 \%$. Another experiment (Section 7.4) investigated the benefit of the sectorization procedure defined in Stage 2 and involved simplifying the process by performing sectorization with default settings and proceeding to Stage 3. This test revealed a considerable loss in service (and other KPIs) in comparison to the proposed framework thus suggesting the benefit introduced by Stage 2 to the overall optimization process. Furthermore, the next experiment (Section 7.5) showed that the assumptions made in Stage 1 in order to overcome the problem of interference estimation were justified. This was confirmed by the results of the experiment which included variation of the 
parameters defined in these assumptions. Lastly, the framework was compared to the traditional way of planning WiMAX networks which has been widely employed in the literature. The results of this experiment (Section 7.6) showed that the proposed solution to WiMAX network planning, which considers the full complexity of the WiMAX architecture is superior to the standard (or traditional) approach when evaluated with the defined set of key performance indicators.

To summarize, the tests conducted in Chapter 7 show that the decomposition approach to the optimization of WiMAX networks proposed in this thesis provides a good framework by solving a specific set of tasks in each stage, and hence simplifying the overall process to achieve better results then when solving the overall problem in a single stage. It also shows that Stage 1 of the optimization framework can be used as a tool for dimensioning purposes and provides more accurate results in comparison to the traditional approaches.

Finally, Chapter 8 presented the evaluation approach in order to validate the WiMAX cell plans using the OPNET Modeler, a leading simulation tool in the industry. Although the performance results from simulation were lower in terms of the system throughput there is a positive correlation (trend) between the optimization results and simulation results (Figure 8.7).

\subsection{Future work}

As WiMAX network planning is an ongoing research problem which introduces new challenges as the technology matures and gets more integrated into the telecommunications industry there are several factors in the proposed optimization framework which can be further improved and complemented in order to enhance it:

- Although current traffic patterns introduced by the triple play services are more DL centric, the shortcoming of the proposed work which considers only DL 
communication can be improved by imposing constraints introduced by the UL.

- As mentioned, with triple or even quadruple play services dominating the entertainment market of broadband services the mix of different applications can be implemented into the modelling part of the work to allow consideration of different QoS requirements.

- The current model considers only a single PBH traffic snapshot, in other words the worst case scenario, which means that the optimization framework strives to build a network design for the most traffic intensive (the most loaded) period of the day. In the real-world scenarios, especially in the case of urban and suburban regions, there can be at least two PBHs with the flow of users from one loaded area of the network to another at one time and visa versa at another time of the day. For example, during the morning hours majority of subscribers are travelling to the city centre for work with lunch time being the busiest time of the broadband service consumption, and travelling to the outskirts of the city (home) in the evening period, introducing the second busiest hour. In order to consider the mobility of users, the dynamic nature of traffic distribution and to improve the cost-efficiency of the network plans multiple traffic snapshots can be employed considering various PBHs, as well as weekly traffic fluctuations, etc.

- Additional modifications can be implemented to the framework to optimize existing networks for expansion purposes.

- In order to gain the maximum profit from the deployed networks the financial model can be modified taking into consideration more complex calculations and predictions, user subscription expansion, and the use of WiMAX technology as a backhaul solution.

- As the WiMAX standard employs SOFDMA which gives a great deal of freedom for service providers, e.g. different channel sizes, the framework can be comple- 
mented by the introduction of multi-channel planning, as well as considering different frequency reuse schemes.

- Other crucial features of WiMAX need to be addressed such as mobility and handoff requirements.

- To achieve maximum spectral and cost efficiency the advanced features of WiMAX, such as MIMO and AAS should be incorporated in the optimization framework.

- As it was mentioned in Section 7.2 the execution time for Stages $1,2 \& 3$ are approximately $15-20 \mathrm{mins}, 1.5 \mathrm{hrs}$ and $2 \mathrm{hrs}$ respectively. Stage 1 is the fastest due to the simplification of the problem, reduction of the decision variables size, and elimination of the slot assignment procedure. The computation time of Stages 2 and 3 can be further improved by the introduction of more efficient techniques to the slot assignment problem. Stage 2 can also employ the technique of parallel computing for cell-by-cell sectorization process.

- And finally the widely predicted integration of WiMAX technology with Wi-Fi can be examined on different deployment scenarios. 


\section{Bibliography}

[1] COST Project 210, Commission of the Eauropean Communities Brussels 1992.

[2] Efficient Millimetre Broadband Radio Access for Convergence and Evolution. European Commission IST project. Embrace (EU FP5, IST-1999-11571). 20002002.

[3] Opnet technologies, http://www.opnet.com.

[4] Visualization toolkit (vtk), http://www.vtk.org.

[5] Wimax capacity white paper, SR Telecom Inc, 2006.

[6] Wimax concepts and RF measurements, White Paper, Agilent Technologies.

[7] Wimax forum, http://www.wimaxforum.org.

[8] Deploying license-exempt wimax solutions. Technical report, Intel Corp. Santa Clara, CA, 2005.

[9] Service recommendations to support technology neutral allocations. In WiMAX forum, 2007.

[10] WiMAX network planning and optimization, chapter 13. Auerbach Publications, 2009.

[11] IEEE Std 802.16-2004. Ieee standard for local and metropolitan area networks part 16: Air interface for fixed broadband wireless access systems. 2004. 
[12] IEEE 802.16a Standard and WiMAX. Igniting broadband wireless access. 2003.

[13] IEEE Std. 802.16e. Ieee standard for local and metropolitan area networks, part 16: Air interface for fixed and mobile broadband wireless access systems, amendment 2: Physical and medium access control layers for combined, fixed and mobile operation in licensed bands and corrigendum 1. 2005.

[14] K.I. Aardal, S.P.M. Van Hoesel, A.M.C.A. Koster, C. Mannino, and A. Sassano. Models and solution techniques for frequency assignment problems. Annals of Operations Research, 153(1):79-129, 2007.

[15] E. Aarts and J. Korst. Simulated annealing and boltzmann machines: a stochastic approach to combinatorial optimization and neural computing. 1988.

[16] V.S. Abhayawardhana, I.J. Wassell, D. Crosby, M.P. Sellars, and M.G. Brown. Comparison of empirical propagation path loss models for fixed wireless access systems. In Vehicular Technology Conference, 2005. VTC 2005-Spring. 2005 IEEE 61st, volume 1, pages 73 - 77 Vol. 1, may-1 june 2005.

[17] R.G. Akl, M.V. Hegde, M. Naraghi-Pour, and P.S. Min. Multicell cdma network design. Vehicular Technology, IEEE Transactions on, 50(3):711-722, 2001.

[18] A.F. Al Rawi, B.S. Sharif, and C.C. Tsimenidis. Pareto-metaheuristic multiobjective network optimization for ofdma-based systems. In Wireless and Mobile Computing, Networking and Communications (WiMob), 2010 IEEE 6th International Conference on, pages 331-336. IEEE, 2010.

[19] S.M. Alamouti. A simple transmit diversity technique for wireless communications. Selected Areas in Communications, IEEE Journal on, 16(8):1451-1458, 1998.

[20] T. Ali-Yahiya, A.L. Beylot, and G. Pujolle. Radio resource allocation in mobile wimax networks using service flows. In Personal, Indoor and Mobile Radio 
Communications, 2007. PIMRC 2007. IEEE 18th International Symposium on, pages 1-5. IEEE, 2007.

[21] S. Allen, LE Hodge, S. Hurley, and R. Whitaker. Estimating the site requirements for $3 \mathrm{~g}$ multimedia services. In Wireless Personal Multimedia Communications, 2002. The 5th International Symposium on, volume 3, pages 1202-1206. IEEE.

[22] SM Allen, S. Hurley, RK Taplin, and RM Whitaker. Automated cell planning to improve network rollout. In Vehicular Technology Conference, 2004. VTC2004Fall. 2004 IEEE 60th, volume 5, pages 3438-3442. IEEE, 2004.

[23] SM Allen, S. Hurley, and RM Whitaker. Automated decision technology for network design in cellular communication systems. In System Sciences, 2002. HICSS. Proceedings of the 35th Annual Hawaii International Conference on, pages 8-pp. IEEE, 2002.

[24] E. Amaldi, A. Capone, and F. Malucelli. Improved models and algorithms for umts radio planning. In Vehicular Technology Conference, 2001. VTC 2001 Fall. IEEE VTS 54th, volume 2, pages 920-924. IEEE, 2001.

[25] E. Amaldi, A. Capone, and F. Malucelli. Planning umts base station location: Optimization models with power control and algorithms. Wireless Communications, IEEE Transactions on, 2(5):939-952, 2003.

[26] E. Amaldi, A. Capone, and F. Malucelli. Radio planning and coverage optimization of $3 g$ cellular networks. Wireless Networks, 14(4):435-447, 2008.

[27] E. Amaldi, A. Capone, F. Malucelli, and C. Mannino. Optimization problems and models for planning cellular networks. Handbook of optimization in telecommunications, pages 917-939, 2006. 
[28] HR Anderson and JP McGeehan. Optimizing microcell base station locations using simulated annealing techniques. In Vehicular Technology Conference, 1994 IEEE 44th, pages 858-862. IEEE, 1994.

[29] J.G. Andrews, A. Ghosh, and R. Muhamed. Fundamentals of WiMAX: understanding broadband wireless networking. Prentice Hall PTR, 2007.

[30] Mishra A.R. Fundamentals of cellular network planning and optimization. Wiley Online Library, 2004.

[31] M.R. Ashayeri and H. Taheri. Mobile wimax capacity estimation in various conditions. In Electrical Engineering (ICEE), 2010 18th Iranian Conference on, pages 483-488. IEEE.

[32] A. Basu and L.N. Frazer. Rapid determination of the critical temperature in simulated annealing inversion. Science, 249(4975):1409, 1990.

[33] E. Biglieri, J. Proakis, and S. Shamai. Fading channels: Information-theoretic and communications aspects. Information Theory, IEEE Transactions on, 44(6):2619-2692, 1998.

[34] S. Boyd, L. Xiao, A. Mutapcic, and J. Mattingley. Notes on decomposition methods. Notes for EE364B, Stanford University, 2007.

[35] H.J. Bremermann. The evolution of intelligence: The nervous system as a model of its environment. University of Washington, Dept. of Mathematics, 1958.

[36] T. Bu, M.C. Chan, and R. Ramjee. Designing wireless radio access networks for third generation cellular networks. In INFOCOM 2005. 24th Annual Joint Conference of the IEEE Computer and Communications Societies. Proceedings IEEE, volume 1, pages 68-78. IEEE, 2005.

[37] P. Calegari, F. Guidec, P. Kuonen, and F. Nielsen. Combinatorial optimization algorithms for radio network planning. Theoretical Computer Science, 263(12):235-245, 2001. 
[38] P. Calegari, F. Guidec, P. Kuonen, and D. Wagner. Genetic approach to radio network optimization for mobile systems. In Vehicular Technology Conference, 1997 IEEE 47th, volume 2, pages 755-759. IEEE, 1997.

[39] G. Cayla, S. Cohen, and D. Guigon. Wimax an efficient tool to bridge the digital divide. In WiMAX Forum, page 2, 2005.

[40] Kah Mun Cheh, Jeffrey B. Goldberg, and Ronald G. Askin. A note on the effect of neighborhood structure in simulated annealing. Computers \& OR, pages 537$547,1991$.

[41] D.T. Chen. On the analysis of using 802.16 e wimax for point-to-point wireless backhaul. In Radio and Wireless Symposium, 2007 IEEE, pages 507-510. IEEE, 2007.

[42] H. Cohn and M. Fielding. Simulated annealing: Searching for an optimal temperature schedule. SIAM Journal on Optimization, 9(3):779, 1999.

[43] G. Colombo and S.M. Allen. A comparison of problem decomposition techniques for the fap. Journal of Heuristics, 16(3):259-288, 2010.

[44] A. Czylwik. Adaptive ofdm for wideband radio channels. In Global Telecommunications Conference, 1996. GLOBECOM'96.'Communications: The Key to Global Prosperity, volume 1, pages 713-718. IEEE, 1996.

[45] E. Damosso. Digital mobile radio towards future generation systems: COST action 231. European Commission, 1999.

[46] F. D'Andreagiovanni and C. Mannino. An optimization model for wimax network planning. wimax network planning and optimization, ed. y. zhang. Auerbach Publications, Boca Raton, USA, 369:386, 2009.

[47] K. Deb. Multi-objective optimization using evolutionary algorithms, volume 16. Wiley, 2001. 
[48] V. Degli-Esposti, F. Fuschini, E. M. Vitucci, and G. Falciasecca. Measurement and modelling of scattering from buildings. Antennas and Propagation, IEEE Transactions on, 55(1):143 -153, jan. 2007.

[49] P. Demestichas, N. Georgantas, E. Tzifa, V. Demesticha, M. Striki, M. Kilanioti, and M. Theologou. Computationally efficient algorithms for location area planning in future cellular systems. Computer Communications, 23(13):1263-1280, 2000 .

[50] RW Eglese. Simulated annealing: a tool for operational research. European journal of operational research, 46(3):271-281, 1990.

[51] A. Eisenblatter, A. Fugenschuh, T. Koch, A. Koster, A. Martin, T. Pfender, O. Wegel, and R. Wessaly. Feasible network configurations for umts. 2002.

[52] A. Eisenblatter and H.F. Geerd. Wireless network design: solution-oriented modeling and mathematical optimization. Wireless Communications, IEEE, 13(6):8-14, 2006.

[53] A. Eisenblatter, H.F. Geerdes, and I. Siomina. Integrated access point placement and channel assignment for wireless lans in an indoor office environment. In World of Wireless, Mobile and Multimedia Networks, 2007. WoWMoM 2007. IEEE International Symposium on a, pages 1-10. IEEE, 2007.

[54] JAN ELLING, M. SØRENSEN, P. MOGENSEN, E. LANG, YU WANG, and O. TEYEB. Mobile broadband network evolution towards 2015-a copenhagen area case study. Month, 10(11):12, 2010.

[55] V. Erceg, L.J. Greenstein, S.Y. Tjandra, S.R. Parkoff, A. Gupta, B. Kulic, A.A. Julius, and R. Bianchi. An empirically based path loss model for wireless channels in suburban environments. Selected Areas in Communications, IEEE Journal on, 17(7):1205-1211, 1999. 
[56] V. Erceg, KVS Hari, MS Smith, D.S. Baum, KP Sheikh, C. Tappenden, JM Costa, C. Bushue, A. Sarajedini, R. Schwartz, et al. Channel models for fixed wireless applications, 2001.

[57] M. Fleischer and S.H. Jacobson. Information theory and the finite-time behavior of the simulated annealing algorithm: Experimental results. INFORMS Journal on Computing, 11(1):35, 1999.

[58] WiMAX Forum. Managing tdd-fdd interference between co-sited base stations deployed in adjacent frequency blocks. Technical report, 2009.

[59] G.J. Foschini. Layered space-time architecture for wireless communication in a fading environment when using multi-element antennas. Bell labs technical journal, 1(2):41-59, 1996.

[60] G.J. Foschini, G.D. Golden, R.A. Valenzuela, and P.W. Wolniansky. Simplified processing for high spectral efficiency wireless communication employing multi-element arrays. Selected Areas in Communications, IEEE Journal on, 17(11):1841-1852, 1999.

[61] B.L. Fox. Integrating and accelerating tabu search, simulated annealing, and genetic algorithms. Annals of Operations Research, 41(2):47-67, 1993.

[62] BROADWAN (EU IST FP6). IPv6 Network Architecture, Mobility, QoS, Multicast. 2003.

[63] A.S. Fraser. Simulation of genetic systems by automatic digital computers ii. effects of linkage on rates of advance under selection. Australian Journal of Biological Sciences, pages 492-499, 1957.

[64] T. Fritsch, K. Tutschku, and K. Leibnitz. Field strength prediction by ray-tracing for adaptive base station positioning in mobile communication networks. ITG FACHBERICHT, pages 67-67, 1995. 
[65] F. Glover. Future paths for integer programming and links to artificial intelligence. Computers \& Operations Research, 13(5):533-549, 1986.

[66] F. Glover et al. Tabu search-part i. ORSA Journal on computing, 1(3):190-206, 1989.

[67] F. Glover, E. Taillard, and E. Taillard. A user's guide to tabu search. Annals of operations research, 41(1):1-28, 1993.

[68] D.E. Goldberg. Genetic algorithms in search, optimization, and machine learning. Addison-wesley, 1989.

[69] A. Gondran, O. Baala, A. Caminada, and H. Mabed. Joint optimization of access point placement and frequency assignment in wlan. In Internet, 2007. ICI 2007. 3rd IEEE/IFIP International Conference in Central Asia on, pages 1-5. IEEE, 2007.

[70] F. Gordejuela-Sánchez, A. Juttner, and J. Zhang. A multiobjective optimization framework for ieee 802.16 e network design and performance analysis. Selected Areas in Communications, IEEE Journal on, 27(2):202-216, 2009.

[71] G. Gougeon, Y. Lostanlen, and L. Maviel. Coupling a deterministic propagation model with diffuse scattering and urban furniture for small cells. In Antennas and Propagation (EUCAP), Proceedings of the 5th European Conference on, pages $3448-3452$, april 2011.

[72] D. Gray. Mobile wimax: A performance and comparative summary. In WiMAX Forum, White Paper, September, 2006.

[73] D. Gray. A comparative analysis of mobile wimax deployment alternatives in the access network. In WiMAX Forum, 2007.

[74] J.W. Greene and K.J. Supowit. Simulated annealing without rejected moves. IEEE Transactions on Computer-Aided Design, 5(1):221-228, 1986. 
[75] V. Gunasekaran and FC Harmantzis. Affordable infrastructure for deploying wimax systems: Mesh v. non mesh. In Vehicular Technology Conference, 2005. VTC 2005-Spring. 2005 IEEE 61st, volume 5, pages 2979-2983. IEEE, 2005.

[76] G. Hampel, K.L. Clarkson, J.D. Hobby, and P.A. Polakos. The tradeoff between coverage and capacity in dynamic optimization of $3 \mathrm{~g}$ cellular networks. In Vehicular Technology Conference, 2003. VTC 2003-Fall. 2003 IEEE 58th, volume 2, pages 927-932. IEEE, 2003.

[77] J.K. Han, B.S. Park, Y.S. Choi, and H.K. Park. Genetic approach with a new representation for base station placement in mobile communications. In Vehicular Technology Conference, 2001. VTC 2001 Fall. IEEE VTS 54th, volume 4, pages 2703-2707. IEEE, 2001.

[78] M. Hata. Empirical formula for propagation loss in land mobile radio services. Vehicular Technology, IEEE Transactions on, 29(3):317 - 325, aug 1980.

[79] JH Holland. Adaptation in natural and artificial systems, 1975.: Univ.

[80] H. Holma, A. Toskala, et al. Wcdma for Umts, volume 4. Wiley Online Library, 2000.

[81] E.J. Hughes. Evolutionary many-objective optimisation: many once or one many? In Evolutionary Computation, 2005. The 2005 IEEE Congress on, volume 1, pages 222-227. IEEE, 2005.

[82] V.B.U.V.M. Hunukumbure and V.S. Vadgama. Radio network dimensioning and planning for wimax networks. FUJITSU Sci. Tech. J, 43(4):435-450, 2007.

[83] S. Hurley. Planning effective cellular mobile radio networks. Vehicular Technology, IEEE Transactions on, 51(2):243-253, 2002.

[84] S. Hurley, DH Smith, and SU Thiel. Fasoft: A system for discrete channel frequency assignment. Radio Science, 32(5):1921-1939, 1997. 
[85] M.F. Iskander and Z. Yun. Propagation prediction models for wireless communication systems. Microwave Theory and Techniques, IEEE Transactions on, 50(3):662-673, 2002.

[86] ITU. ITU-R Reccommendation P 452-10, Prediction procedure for the evaluation of interference between stations on the surface of the Earth at frequencies above about $0.1 \mathrm{GHz}$.

[87] ITU. ITU-R Reccommendation P 526-7, Propagation by diffraction. 2001.

[88] S.B. Jamaa, Z. Altman, J.M. Picard, B. Fourestié, and J. Mourlon. Manual and automatic design for umts networks. Mobile Networks and Applications, 9(6):619-626, 2004.

[89] J. Jang and K.B. Lee. Transmit power adaptation for multiuser ofdm systems. Selected Areas in Communications, IEEE Journal on, 21(2):171-178, 2003.

[90] K. Kim, Y. Han, and S.L. Kim. Joint subcarrier and power allocation in uplink ofdma systems. Communications Letters, IEEE, 9(6):526-528, 2005.

[91] S. Kirkpatrick, C.D. Gelatt, and M.P. Vecchi. Optimization by simulated annealing. science, 220(4598):671, 1983.

[92] D. Kivanc, G. Li, and H. Liu. Computationally efficient bandwidth allocation and power control for ofdma. Wireless Communications, IEEE Transactions on, 2(6):1150-1158, 2003.

[93] B. Krishnamachari and S.B. Wicker. Experimental analysis of local search algorithms for optimal base station location. In Proceedings of International Conference on Evolutionary Computing for Computer, Communication, Control and Power. Chennai, India, 2000.

[94] B. Christophe F. Laurence L. Philippe, B. Ditrich. Wimax, making ubiquitous high-speed data services a reality. Alcatel Strategy White Paper, 2004. 
[95] J. Laiho, A. Wacker, T. Novosad, and Inc Books24x7. Radio network planning and optimisation for UMTS. Wiley Online Library, 2002.

[96] C.Y. Lee and H.G. Kang. Cell planning with capacity expansion in mobile communications: A tabu search approach. Vehicular Technology, IEEE Transactions on, 49(5):1678-1691, 2000.

[97] R. Leese and S. Hurley. Methods and algorithms for radio channel assignment, volume 23. Oxford University Press, USA, 2002.

[98] G. Li and H. Liu. Downlink radio resource allocation for multi-cell ofdma system. Wireless Communications, IEEE Transactions on, 5(12):3451-3459, 2006.

[99] X. Li. Radio Access Network Dimensioning for 3G UMTS. Vieweg+ Teubner Verlag, 2010.

[100] P. Lin, H. Ngo, C.M. Qiao, X. Wang, T. Wang, and D.Y. Qian. Minimum cost wireless broadband overlay network planning. In Proceedings of the 2006 International Symposium on on World of Wireless, Mobile and Multimedia Networks, pages 228-236. IEEE Computer Society, 2006.

[101] MD Louta, PP Demestichas, ED Loutas, SK Kraounakis, ME Theologou, and ME Anagnostou. Cost-efficient design of future broadband fixed wireless access systems. Wireless Personal Communications, 27(1):57-87, 2003.

[102] T. Imai M. Ghoraishi, J. Takada. Radio wave scattering from lampposts in microcell urban mobile propagation channel. ECTI transactions on Electrical engineering, Electronics and Communications, 7(1), February 2009.

[103] C. Mannino, F. Rossi, and S. Smriglio. The network packing problem in terrestrial broadcasting. OPERATIONS RESEARCH-BALTIMORE THEN LINTHICUM-, 54(4):611, 2006.

[104] Masood Maqbool. Subcarrier permutation (ieee 802.16e). Master's thesis, Telecom Paris, 2007. 
[105] R. Mathar and T. Niessen. Optimum positioning of base stations for cellular radio networks. Wireless Networks, 6(6):421-428, 2000.

[106] R. Mathar and M. Schmeinck. Optimization models for gsm radio. International Journal of Mobile Network Design and Innovation, pages 70-75, 2005.

[107] N. Metropolis, A.W. Rosenbluth, M.N. Rosenbluth, A.H. Teller, E. Teller, et al. Equation of state calculations by fast computing machines. The journal of chemical physics, 21(6):1087, 1953.

[108] J. Milanovic, S. Rimac-Drlje, and K. Bejuk. Comparison of propagation models accuracy for wimax on 3.5 ghz. In Electronics, Circuits and Systems, 2007. ICECS 2007. 14th IEEE International Conference on, pages 111-114. IEEE.

[109] A. Molina, GE Athanasiadou, and AR Nix. The automatic location of basestations for optimised cellular coverage: A new combinatorial approach. In Vehicular Technology Conference, 1999 IEEE 49th, volume 1, pages 606-610. IEEE, 1999.

[110] D. Molkdar, S. Burley, and J. Wallington. Comparison between simulation and analytical methods of umts air interface capacity dimensioning. In Vehicular Technology Conference, 2002. Proceedings. VTC 2002-Fall. 2002 IEEE 56th, volume 3, pages 1596-1601. IEEE, 2002.

[111] P. Moscato. An introduction to population approaches for optimization and hierarchical objective functions: A discussion on the role of tabu search. Annals of Operations Research, 41(2):85-121, 1993.

[112] D. Nam and C.H. Park. Multiobjective simulated annealing: A comparative study to evolutionary algorithms. International Journal of Fuzzy Systems, 2(2):87-97, 2000.

[113] L. Nuaymi, N. Bouida, N. Lahbil, and P. Godlewski. Headers overhead estimation, header suppression and header compression in wimax. In Wireless 
and Mobile Computing, Networking and Communications, 2007. WiMOB 2007. Third IEEE International Conference on, pages 17-17. IEEE, 2007.

[114] FA Ogbu and DK Smith. The application of the simulated annealing algorithm to the solution of the $\mathrm{n} / \mathrm{m} / \mathrm{cmax}$ flowshop problem. Computers \& Operations Research, 17(3):243-253, 1990.

[115] Kathryn E. Oliver. UMTS Network Optimization. PhD thesis, Cardiff University, 2005.

[116] K.E. Oliver, S.M. Allen, and S. Hurley. Utilising network dimensioning to improve automated cell planning. In Vehicular Technology Conference, 2004. VTC2004-Fall. 2004 IEEE 60th, volume 5, pages 3419-3423. IEEE.

[117] M. Paolini. Mobile wimax: The best personal broadband experience! In WiMAX forum, 2006.

[118] T.S. Rappaport and Safari Books Online (Firme). Wireless communications: principles and practice, volume 2. Prentice Hall PTR New Jersey, 1996.

[119] M.M.G. Rashed, S.U.M. Joni, and M.M.I. Siddique. Radio network planning for fixed wimax in dhaka city and it's performance analysis. In Computer and Information Technology (ICCIT), 2010 13th International Conference on, pages 589-593. IEEE, 2010.

[120] I. Recommendation. 1225, guidelines for evaluation of radio transmission technologies for imt-2000,'. Int. Telecommun. Union (ITU), 1997.

[121] F. Romeo and A. Sangiovanni-Vincentelli. A theoretical framework for simulated annealing. Algorithmica, 6(1):302-345, 1991.

[122] P. Serafini. Simulated annealing for multiple objective optimization problems. In G.H. Tzeng, H.F. Wang, V. P. Wen, and P.L. Yu, editors, Multiple Criteria Decision Making. Expand and Enrich the Domains of Thinking and Application, pages 283-292. Springer Verlag, 1994. 
[123] DH Smith, SM Allen, S. Hurley, and WJ Watkins. Frequency assignment: Methods and algorithms. In Proceedings of the NATO RTA SET/ISET Symposium on Frequency Assignment, Sharing and Conservation in Systems (Aerospace), pages K1-K18, 1998.

[124] G. Smyth. Wireless technologies and e-learning: Bridging the digital divide. Intel Corporation, December, 2006.

[125] C. So-In, R. Jain, and A.K. Tamimi. Capacity evaluation for ieee 802.16 e mobile wimax. Journal of Computer Systems, Networks, and Communications, 2010:1-1, 2010.

[126] R. Steele and L. Hanzo. Mobile radio communications, volume 3. Pentech Press, 1992.

[127] Z. Sun, Y. Zhou, M. Peng, and W. Wang. Dynamic resource allocation with guaranteed diverse qos for wimax system. In Communications, Circuits and Systems Proceedings, 2006 International Conference on, volume 2, pages 13471351. IEEE, 2006.

[128] H. Szu and R. Hartley. Fast simulated annealing* 1. Physics letters A, 122(34):157-162, 1987.

[129] RK Taplin, DM Ryan, SM Allen, S. Hurley, and NJ Thomas. Algorithms for the automatic design of wimax networks. In Next Generation Mobile Applications, Services and Technologies, 2007. NGMAST'07. The 2007 International Conference on, pages 322-327. IEEE, 2007.

[130] V. Tarokh, H. Jafarkhani, and A.R. Calderbank. Space-time block codes from orthogonal designs. Information Theory, IEEE Transactions on, 45(5):14561467, 1999. 
[131] T. Theodoros and V. Kostantinos. Wimax network planning and system's performance evaluation. In Wireless Communications and Networking Conference, 2007. WCNC 2007. IEEE, pages 1948-1953. IEEE, 2007.

[132] C. Tovey. Simulated simulated annealing. American Journal of Mathematical and Management Sciences, 8(3):389-407, 1988.

[133] K. Tutschku. Demand-based radio network planning of cellular mobile communication systems. In INFOCOM'98. Seventeenth Annual Joint Conference of the IEEE Computer and Communications Societies. Proceedings. IEEE, volume 3, pages 1054-1061. IEEE, 1998.

[134] K. Tutschku and K. Leibnitz. Fast ray-tracing for field strength prediction in cellular mobile network planning. In Vehicular Technology Conference, 1996.'Mobile Technology for the Human Race'., IEEE 46th, volume 1, pages 541-545. IEEE, 1996.

[135] EL Ulungu, J. Teghem, PH Fortemps, and D. Tuyttens. Mosa method: a tool for solving multiobjective combinatorial optimization problems. Journal of MultiCriteria Decision Analysis, 8(4):221-236, 1999.

[136] M. Vasquez and J.K. Hao. A heuristic approach for antenna positioning in cellular networks. Journal of Heuristics, 7(5):443-472, 2001.

[137] R.M. Whitaker, S.M. Allen, and S. Hurley. Efficient offline coverage and load evaluation for cdma network modeling. Vehicular Technology, IEEE Transactions on, 58(7):3704-3712, 2009.

[138] R.M. Whitaker and S. Hurley. Evolution of planning for wireless communication systems. In System Sciences, 2003. Proceedings of the 36th Annual Hawaii International Conference on, pages 10-pp. IEEE, 2003.

[139] R.M. Whitaker and S. Hurley. Omni-directional cell planning. Telecommunications Network Design and Management, pages 25-41, 2003. 
[140] R.M. Whitaker and S. Hurley. On the optimality of facility location for wireless transmission infrastructure. Computers \& industrial engineering, 46(1):171$191,2004$.

[141] R.M. Whitaker, S. Hurley, and S.M. Allen. Sensitivity of service coverage evaluation for wcdma systems. In Wireless Telecommunications Symposium, 2007. WTS 2007, pages 1-10. IEEE, 2007.

[142] R.M. Whitaker, S. Hurley, and S.M. Allen. Modeling cell placement and coverage for heterogeneous 3g services. Wireless Technology, pages 241-256, 2009.

[143] R.M. Whitaker, L. Raisanen, and S. Hurley. The marginal cost of service provision in cellular networks. In System Sciences, 2005. HICSS'05. Proceedings of the 38th Annual Hawaii International Conference on, pages 304b-304b. IEEE.

[144] R.M. Whitaker, L. Raisanen, and S. Hurley. The infrastructure efficiency of cellular wireless networks. Computer networks, 48(6):941-959, 2005.

[145] M. WiMAX. Part ii: A comparative analysis. In Copyright WIMAX Forum, 2006.

[146] M. WiMAX-Part. I: A technical overview and performance evaluation. In WiMAX Forum, 2006.

[147] D.H. Wolpert and W.G. Macready. No free lunch theorems for search. Technical report, Citeseer, 1995.

[148] H. Yaghoobi. Scalable ofdma physical layer in ieee 802.16 wirelessman. 2004.

[149] J. Yang, ME Aydin, J. Zhang, and C. Maple. Umts base station location planning: a mathematical model and heuristic optimisation algorithms. Communications, IET, 1(5):1007-1014, 2007. 
[150] J. Yang, J. Zhang, M.E. Aydin, and J.Y. Wu. A novel programming model and optimisation algorithms for wcdma networks. In Vehicular Technology Conference, 2007. VTC2007-Spring. IEEE 65th, pages 1182-1187. IEEE, 2007.

[151] J. Yang, J. Zhang, M.E. Aydin, and J.Y. Wu. Optimisation of wcdma radio networks with consideration of link-level performance factors. International Journal of Mobile Network Design and Innovation, 2(1):26-33, 2007.

[152] H. Yin and S. Alamouti. Ofdma: A broadband wireless access technology. In Sarnoff Symposium, 2006 IEEE, pages 1-4. IEEE, 2006.

[153] H. Yin and H. Liu. An efficient multiuser loading algorithm for ofdm-based broadband wireless systems. In Global Telecommunications Conference, 2000. GLOBECOM'O0. IEEE, volume 1, pages 103-107. IEEE, 2000.

[154] J. Zimmermann, R. Höns, and H. Mühlenbein. Encon: an evolutionary algorithm for the antenna placement problem. Computers \& industrial engineering, 44(2):209-226, 2003. 